Florida International University FIU Digital Commons

\title{
Conjugated Polymer-Based Biomaterials Through Controlled Self-Assembly
}

Megan Twomey

Florida International University, mtwom001@fiu.edu

DOI: $10.25148 /$ etd.FIDC000272

Follow this and additional works at: https://digitalcommons.fiu.edu/etd

Part of the Biochemistry Commons, Biotechnology Commons, Nanotechnology Commons, Organic Chemistry Commons, and the Polymer Chemistry Commons

\section{Recommended Citation}

Twomey, Megan, "Conjugated Polymer-Based Biomaterials Through Controlled Self-Assembly" (2016). FIU Electronic Theses and Dissertations. 2452.

https://digitalcommons.fiu.edu/etd/2452 


\section{FLORIDA INTERNATIONAL UNIVERSITY}

Miami, Florida

\section{CONJUGATED POLYMER-BASED BIOMATERIALS THROUGH CONTROLLED SELF- ASSEMBLY}

A dissertation submitted in partial fulfillment of the

requirements for the degree of

DOCTOR OF PHILOSOPHY

in

CHEMISTRY

by

Megan Twomey

2016 
To: $\quad$ Dean Michael R. Heithaus

College of Arts, Sciences and Education

This dissertation, written by Megan Twomey, and entitled Conjugated Polymer-Based Biomaterials Through Controlled Self-Assembly, having been approved in respect to style and intellectual content, is referred to you for judgment.

We have read this dissertation and recommend that it be approved.

Konstantinos Kavallieratos

Watson Lees

Anthony McGoron

Kevin O'Shea

Joong Ho Moon, Major Professor

Date of Defense: March 9, 2016

The dissertation of Megan Twomey is approved.

Dean Michael R. Heithaus College of Arts, Sciences and Education

Andrés G. Gil Vice President for Research and Economic Development and Dean of the University Graduate School

Florida International University, 2016 
(C) Copyright 2016 by Megan Twomey

All rights reserved. 


\section{DEDICATION}

I dedicate this dissertation to my family for all of their love and support. I would not be as ambitious or successful without your encouragement. I love you all. 


\section{ACKNOWLEDGMENTS}

I would like to thank my advisor, Dr. Joong Ho Moon for his guidance and support. You pushed me when I needed to be pushed and always encouraged me to be a better student and scientist. I would like to thank my committee members: Dr. Konstantinos Kavallieratos, Dr. Watson Lees, Dr. Anthony McGoron, and Dr. Kevin O’Shea for serving on my committee and providing useful feedback to tackle all of my graduate school hurdles.

I would like to thank all of the past and present lab mates, there have been many of you. Tereza Vokata for reminding me of deadlines, reading all of my papers and giving me feedback and advice. Your endless supply of polymers helped me hone my CPN fabrication skills and complete my CPN arrays. Eladio Mendez, my brother from another mother, your wisdom is boundless and thank you for bestowing upon me knowledge of chemistry and life. Special thanks for being $\mathrm{n}=1$ for the Moon lab and paving the way. Prakash Manandhar for your supply of polymers, monomers, and Nepalese spices. Best of luck to you during the rest of your $\mathrm{PhD}$, I will miss our coffee breaks and shooting the breeze with you. Thanks to the past post docs Rajib Choudhury and Rajesh Kumar for letting me collaborate with you during your time in the Moon lab. Thanks to all the past and present graduate students: Salauddin Ahmed, Young Myoung, Michelle Pierre, and Heather Kumar. Keep on trucking. Thanks to past undergraduates Zahilyn Roche, Giselle Gomez, and Christian Machado for setting the bar too high for a smart and sassy youth. Thanks to past lab techs Yoonmi Na and Youri Lee, and for showing me the important cell stuff.

I would like to thank the Department of Chemistry for providing me funding for four years through teaching assistantships and the guidance of Dr. Palmer Graves. I would also like to thank the University Graduate School for awarding me the Dissertation Year Fellowship (DYF), which has afforded me funding and most importantly, the time during my last year to complete this dissertation.

I would like to thank all of my friends near and far for support and debauchery. The "Soup There It Is" crew for the trivial fun. To all of my intramural teammates from McGlovin, Bitchin Ballers, and Mad Gatos, thanks for the competitive fun and all the championships. Special thanks to my dear friends Mike Hyre and Sheila Vahkaria, I love you both and I cherish the time spent in Miami, and hope to be reunited in the same city one 
day. Maria "B" Campana, you have always been there to remind that I was more than capable to do this Ph.D. and to always keep going. Your support was unwavering and I can't thank you enough for that. Jim O’Leary and Sasha Wagner (The Jimsha), you guys set the bar on being the "cool couple" and I enjoyed all the good times we had in Miami, despite the humidity. Kristi Ray, you have come into my life towards the end of my Ph.D., and I'm not quite sure I would have completed the actual writing and completion of this dissertation. Thank you for giving me the final push I needed to get it done. Thanks to all my fellow grad students that I've had the pleasure to meet, and spend time with "in the bunker" that is grad school.

Lastly, I would like to express my deepest gratitude to my parents, my mother Jean and step-father Mark, and my brother, Chris. You have shown me there are no challenges or difficulties in life that cannot be overcome. I thank you for always being supportive and unconditional love, and most importantly, understanding during my time as a Ph.D. student. Thank you to the rest of my family (there are many of you) for providing me a wonderful family environment to grow as a person. 


\section{ABSTRACT OF THE DISSERTATION}

\section{CONJUGATED POLYMER-BASED BIOMATERIALS THROUGH CONTROLLED SELF-}

\section{ASSEMBLY}

by

Megan Twomey

Florida International University, 2016

Miami, Florida

Professor Joong Ho Moon, Major Professor

Synthetic polymeric materials have gained significant use as biological materials (biomaterials) in biomedical and pharmaceutical applications. As a result, a demand for well-defined polymers with tunable properties has emerged. The synthetic versatility of polymeric biomaterials allows the opportunity to understand the structure-property relationship of materials and their cellular interactions. A novel class of polymeric biomaterials are conjugated polymers (CPs), which possess desirable physicochemical and excellent photophysical properties, including inherent fluorescence. The synthetic versatility of CPs allows easy modification of the conjugated backbone to tune emission and side chain structures to adjust biocompatibility through increased water solubility, controlled biodegradability, and incorporation of targeting units. The aim of this dissertation is to better understand conjugated polymer nanoparticle (CPN) structure and self-assembly in an aqueous environment, and how those structural features affect cellular interactions to establish a structure-function relationship.

This work presents the fabrication of several different CPNs for cancer cell targeting and labelling, and differentiation of biologically important molecules. Core-shell nanoparticles were prepared using a semiflexible cationic CPN complexed with hyaluronic acid (HA), a polyanion. The resulting CPNs exhibited high cancer cell specificity with low adsorption to normal cells, as a result of HA's affinity towards overexpressed receptors on cancer cell surface. A systematic investigation on the aggregation properties of CPNs that vary by side chain and backbone structures in response to different biologically important anionic polysaccharides in a complex biological medium was conducted. Mitochondria-specific CPNs were fabricated from a semiflexible CPN modified with the mitochondrial-targeting triphenylphosphonium (TPP) group. The subcellular 
localization and cellular toxicity were dependent on backbone flexibility, hydrophilicity, and molecular weight. Dual-targeting CPNs grafted with folic acid (FA) side chains and complexed with hyaluronic acid (HA) were fabricated for improved uptake and bioimaging of cancer cells.

The work presented here shows how modifications to CPN backbone and side chain structures modulate their cellular interactions. The physicochemical and biophysical properties of CPNs affect biocompatibility and understanding those properties will lead to the development of novel CP-based biomaterials. 


\section{TABLE OF CONTENTS}

CHAPTER

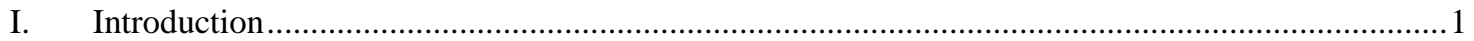

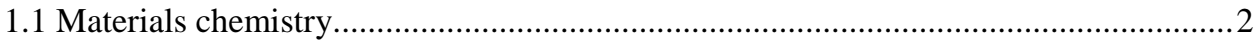

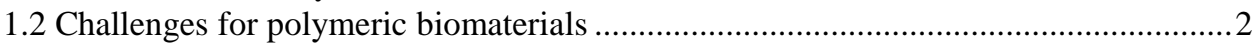

1.3 Conjugated polymers ……………...............................................................................

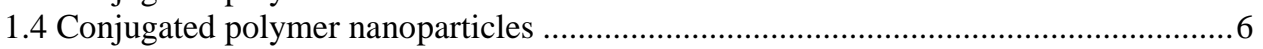

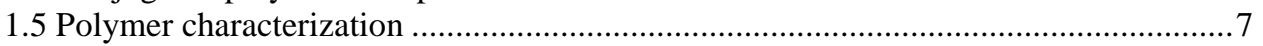

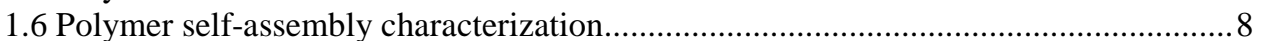

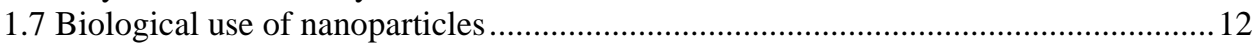

1.8 Characterization of cellular interactions …….......................................................... 15

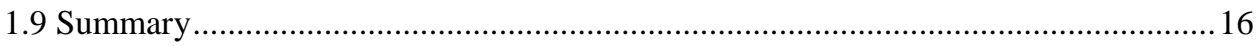

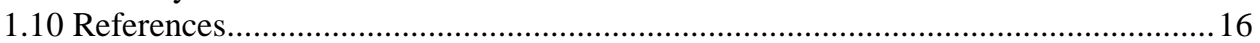

II. Fabrication of core-shell nanoparticles via controlled aggregation of semi-flexible conjugated polymer and hyaluronic acid ...................................................................................19

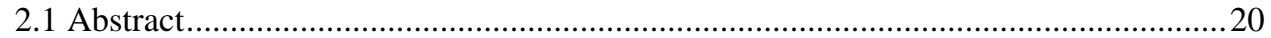

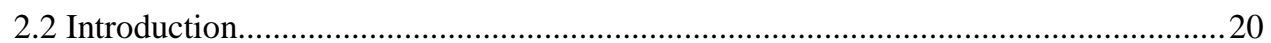

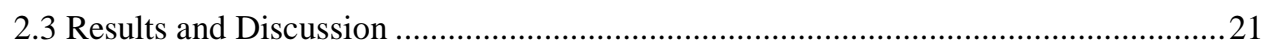

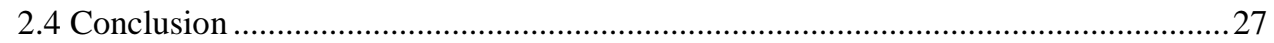

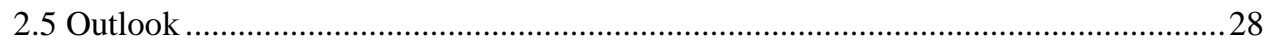

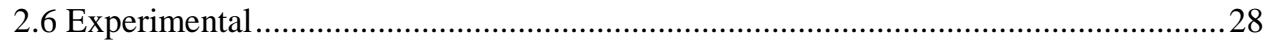

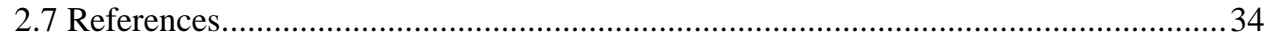

III. Differential interactions of conjugated polymer nanoparticles with glycosaminoglycans in

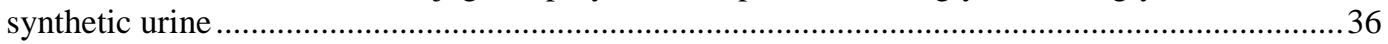

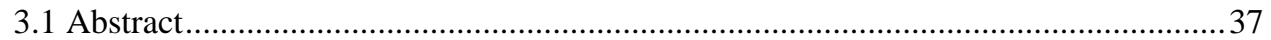

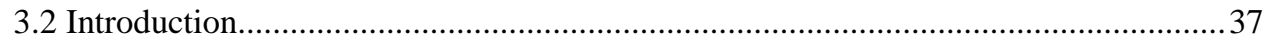

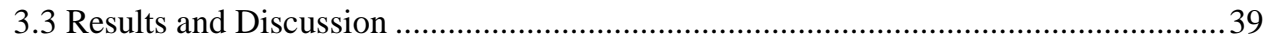

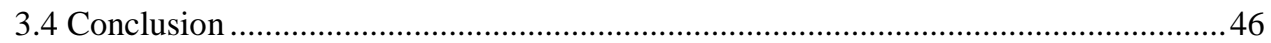

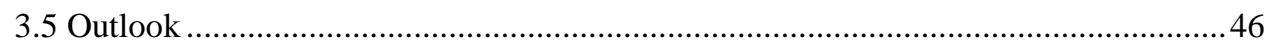

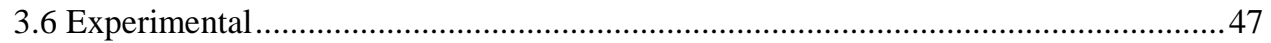

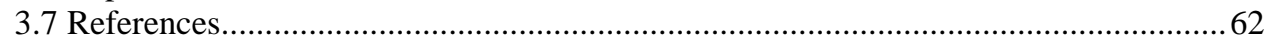

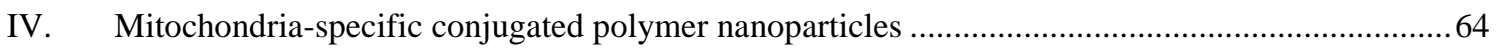

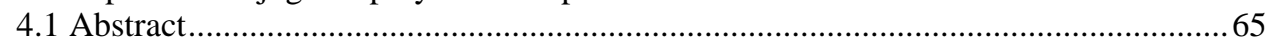

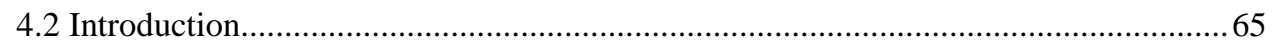

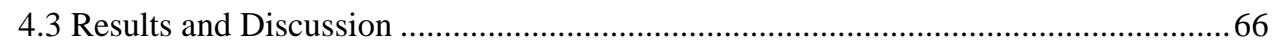

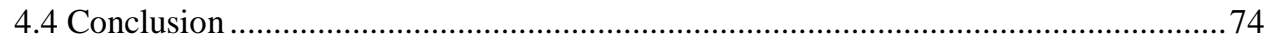

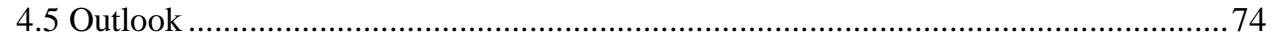

4.6 Experimental ............................................................................................................. 75

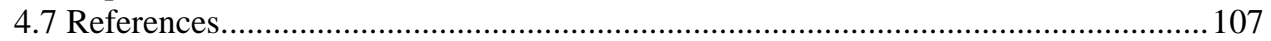

V. Dual targeting conjugated polymer nanoparticles of folate and hyaluronan receptors for

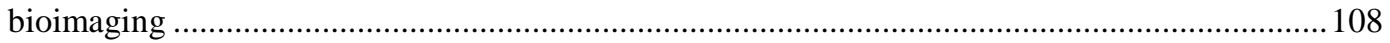

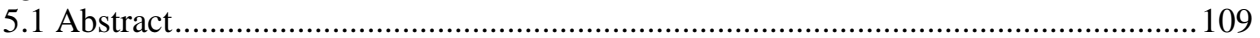

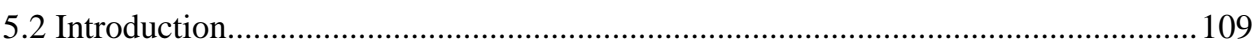

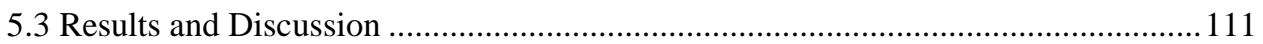




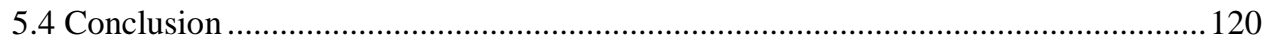

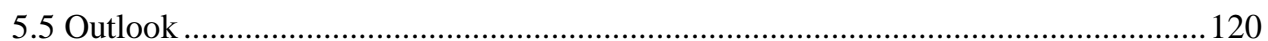

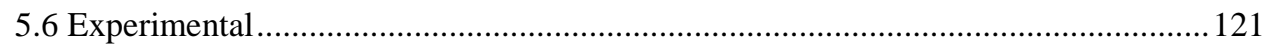

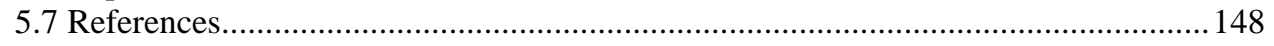

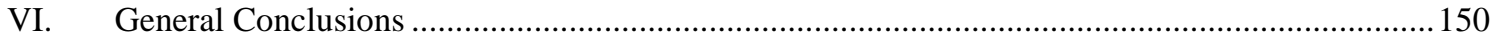

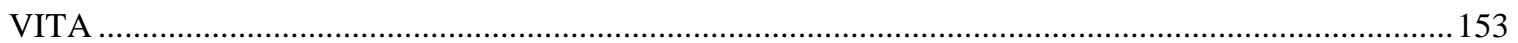




\section{LIST OF TABLES}

TABLE

PAGE

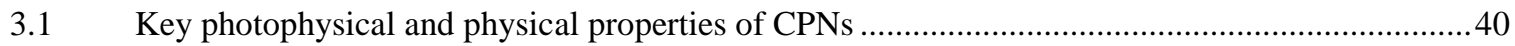

S3.1 Summary of NTA data for CPN/GAG complexes ...................................................................60

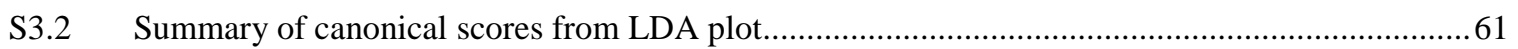

S3.3 Squared Mahalanobis distances to each group centroid..................................................................61

S4.1 Hydrodynamic diameters and zeta potential of CPNs.................................................................97 


\section{LIST OF FIGURES}

FIGURE

PAGE

1.1 Common examples of conjugated polymers 3

Example configuration of delocalized electrons in a $\pi$ conjugated system...............................

Sonogashira and Glaser coupling for PPE and PPB synthesis ................................................

1.4 Jablonski diagram showing processes when a molecule is irradiated by light ............................6

Example GPC chromatogram illustrating molecular weight distribution ................................... 7

1.6 Schematic representation of the spectral shift in $\mathrm{H}$ and J-aggregates based on

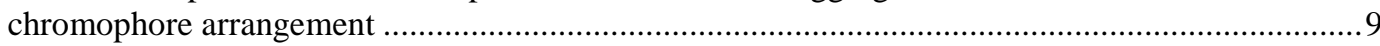

1.7 Schematic representation of zeta potential of a negatively charged particle.....

1.8 Schematic representation of the enhanced retention and permeability effect (EPR) that occurs in diseased tissue

2.1 Chemical structure of the semi-flexible PPB and schematic presentation of structural reorganization of the semi-flexible PPB upon HA complexation

2.2 Absorbance and emission spectra of semi-flexible PPB and rigid PPE before and after HA complexation

2.3 AFM topographic (a, c) and phase (b, d) images of the semi-flexible PPB (a, b) and semi-flexible PPB/HA nanoparticles formed at 1:3 molar ratio (c, d). The semi-flexible $\mathrm{PPB} / \mathrm{HA}$ exhibits elongated particles on a mica surface (c), and the phase image (d) reveals that the complexes are core--shell nanoparticles.

2.4 Topography and phase images and size analysis histograms of semi-flexible PPB, semi-flexible PPB/HA (1:1), and semi-flexible PPB/HA (1:3).

2.5 Normalized fluorescent mean intensity ratios of various cells treated with the semi-flexible PPB/HA (1:3) up to $3 \mathrm{~h}$.

2.6 Fluorescent microscopic and contrast images of HeLa/HEK mixed cells, HEK cells, and HeLa cells incubated with the semi-flexible PPB/HA for $1 \mathrm{~h}$.

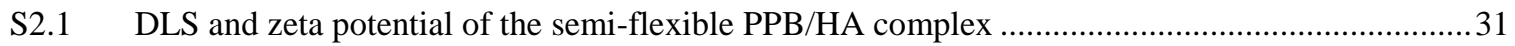

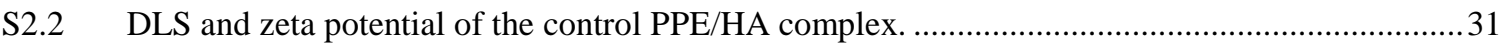

S2.3 Topography and phase images, size distribution, and height histograms for control PPE/HA.......32

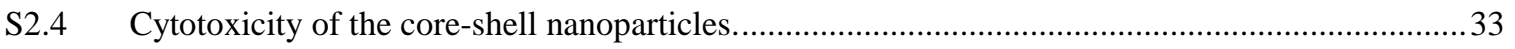

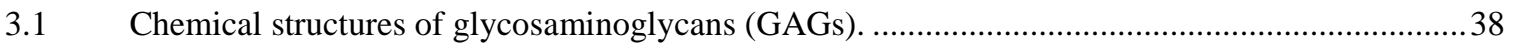

3.2 Chemical structures of CPs with different side chains (P1 and P2), PPE with flexible backbone (P3), and PPB with flexible backbone (P4). 39 
3.3 Average hydrodynamic diameters of CPN-1, CPN-2, CPN-3, and CPN-4 upon complexation with hyaluronic acid, chondroitin sulfate, dermatan sulfate, and heparin sulfate

3.4 Average size distributions of CPNs complexed with GAGs measured by NTA .......................41

3.5 Effect of GAG complexation on absorption and emission for CPN-1, CPN-2, CPN-3, and CPN-4 in water.

3.6 Hierarchical cluster analysis (HCA) of CPN-2+GAGs in water...........................................44

3.7 Emission spectra of CPN-1, CPN-2, CPN-3, and CPN-4 in the presence of GAG-containing

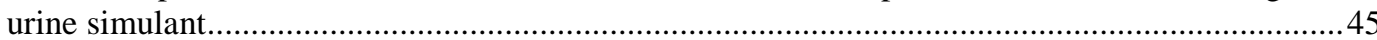

3.8 An LDA plot between two largest canonical correlations of emission intensity ratios of CPNs

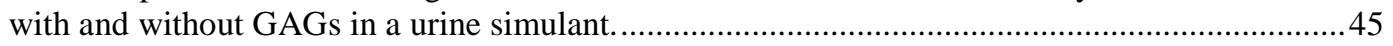

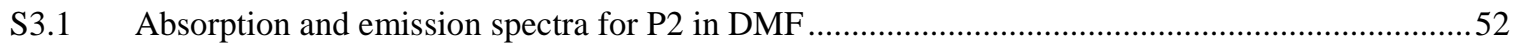

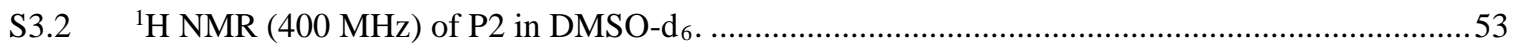

S3.3 Absorption and emission spectra for $\mathrm{CPN}-2$ in water. ..........................................................53

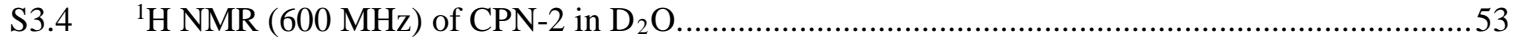

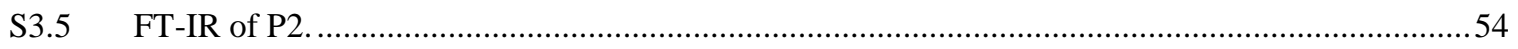

S3.6 Absorption and emission spectra for P3 in DMF. .......................................................... 54

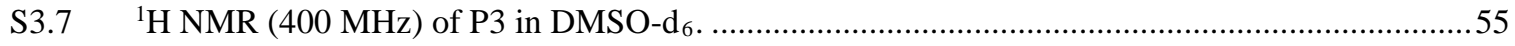

S3.8 Absorption and emission spectra for $\mathrm{CPN}-3$ in water. .........................................................55

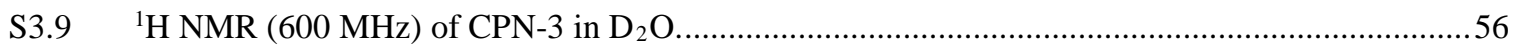

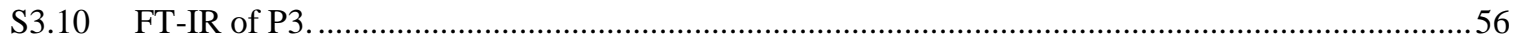

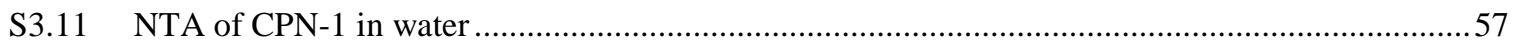

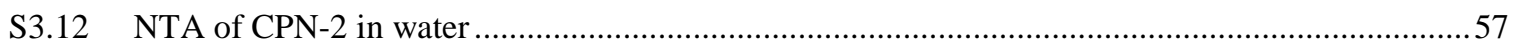

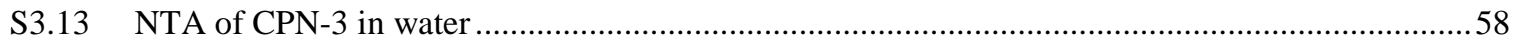

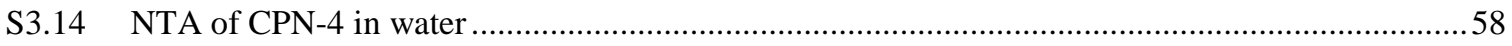

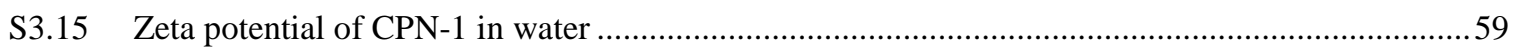

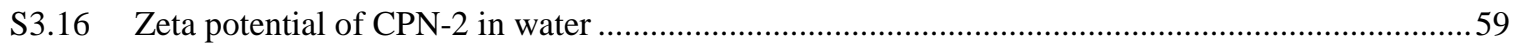

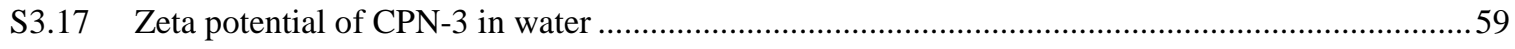

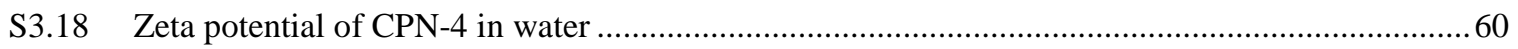


4.1 Chemical structures of PPEs with (PPE-1) and without (PPE-2) biodegradable linkers in the backbone and a schematic illustration of cellular entry and mitochondria localization of biodegradable CPNs. CPN-1and CPN-2 were fabricated with the corresponding PPEs via self-assembly in water.

4.2 Microscopic images of HeLa cells incubated with CPNs for $18 \mathrm{~h}$ followed by mitochondria and nuclear staining.

4.3 Confocal microscopic images of HeLa cells incubated with CPN-1 $0.2 \mu \mathrm{M}$ and $2 \mu \mathrm{M}$ for $18 \mathrm{~h}$ followed by mitochondria staining.

4.4 Absorption and emission spectra of CPN-1 treated with $10 \mathrm{mM}$ glutathione (GSH) for 0 and $18 \mathrm{~h}$ at $37^{\circ} \mathrm{C}$.......

4.5 Microscopic images of HeLa cells incubated with CPN-3 for $18 \mathrm{~h}$ followed by mitochondrial and nuclear staining

4.6 Confocal microscopic images of HeLa cells incubated with CPN/HA for 18 h followed by mitochondria and nucleus staining..

4.7 Fluorescence analysis of mitochondrial and cytosolic fractions of HeLa cells incubated with CPNs

Endocytosis inhibition study under pharmacological inhibitors treatments ..............................73

4.9 Time-course localizations of A) $\mathrm{CPN}-1$ and B) $\mathrm{CPN}-2$ on endosomes and mitochondria..............74

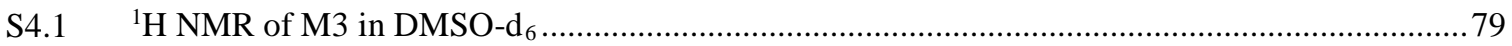

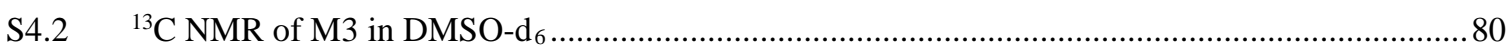

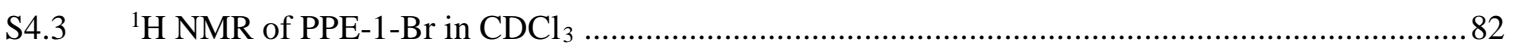

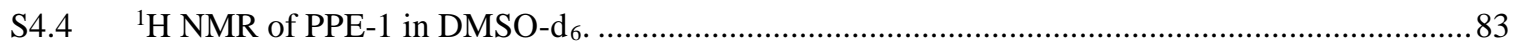

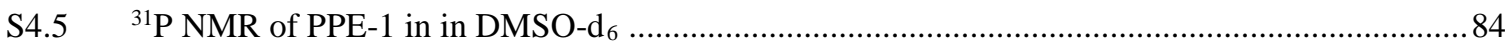

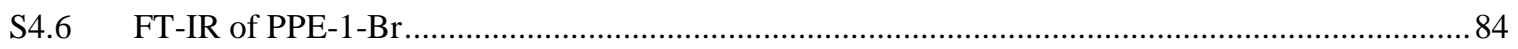

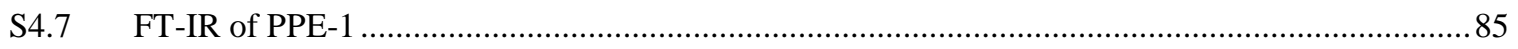

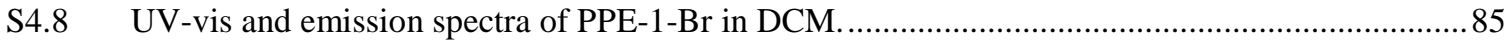

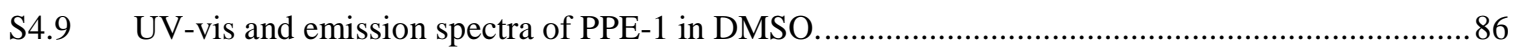

S4.10 UV-vis and emission spectra of CPN-1 1\% DMSO in water (v/v).....................................86

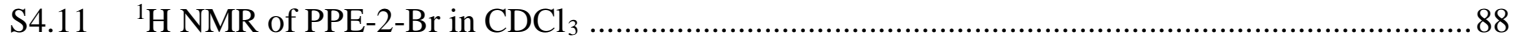

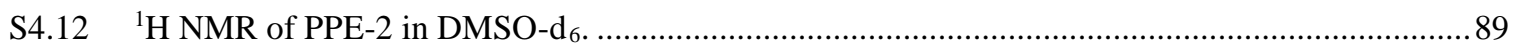

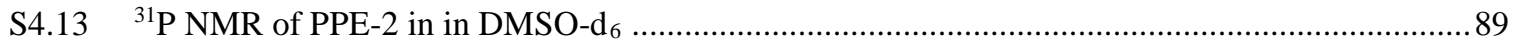




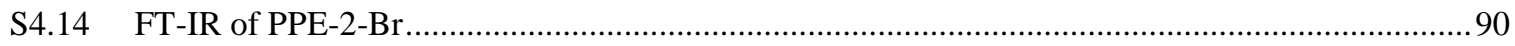

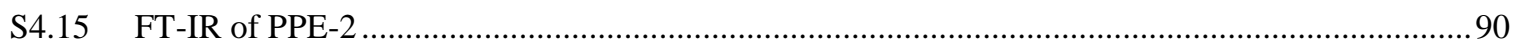

S4.16 UV-vis and emission spectra of PPE-2-Br in DCM................................................................

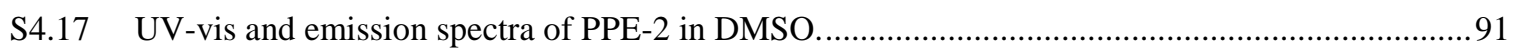

S4.18 UV-vis and emission spectra of CPN-2 1\% DMSO in water (v/v)............................................92

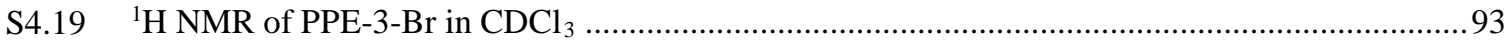

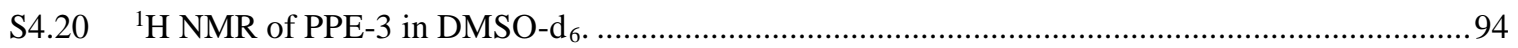

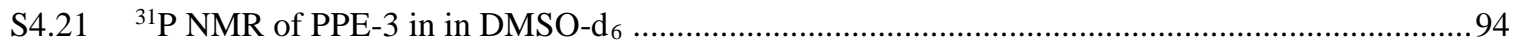

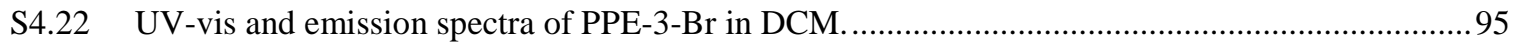

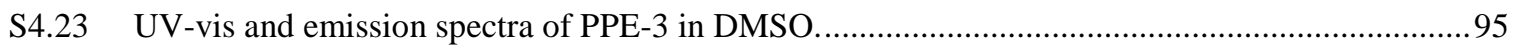

S4.24 UV-vis and emission spectra of CPN-3 1\% DMSO in water (v/v)...........................................96

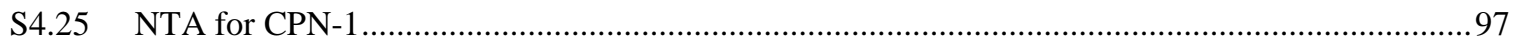

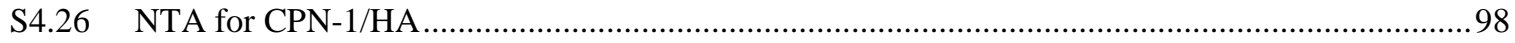

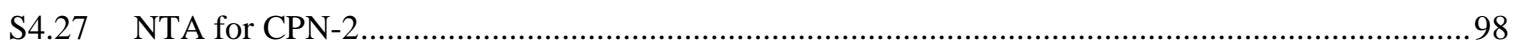

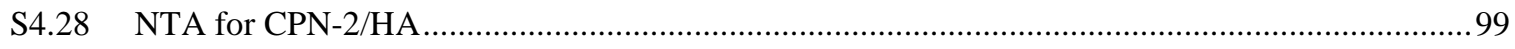

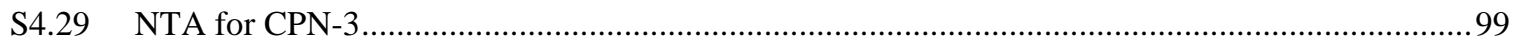

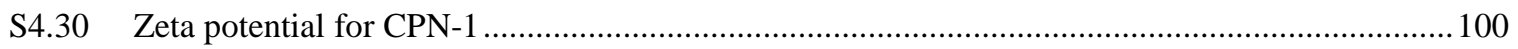

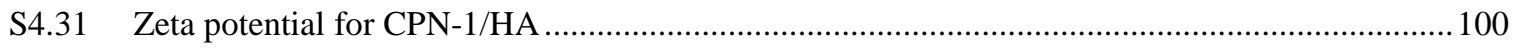

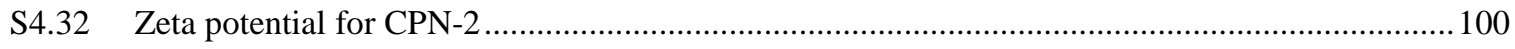

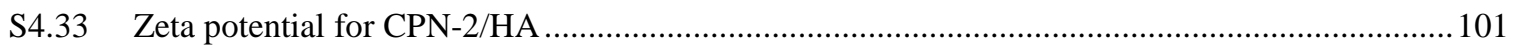

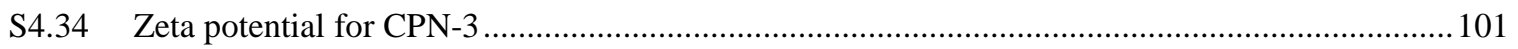

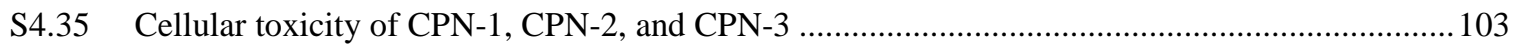

S4.36 Microscopic images of HeLa cells incubated with CPN-2 for $18 \mathrm{~h}$ followed by endosome

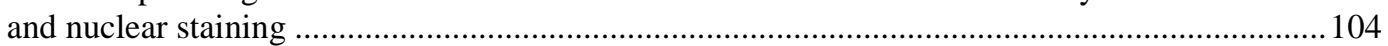

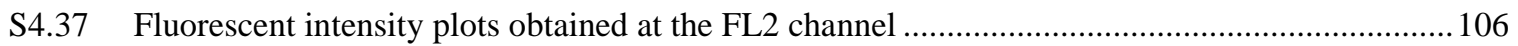

5.1 Chemical structures CPNs grafted with folic acid-modified PEG $2000\left(\mathrm{CPN}_{2} \mathrm{PEG}_{2000}-\mathrm{FA}\right)$ and

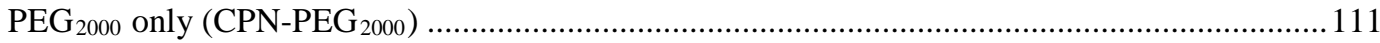

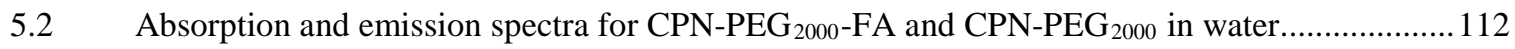


5.3 Cellular uptake of CPN-PEG $2000-F A$ and CPN-PEG 2000 complexed with $40 \mathrm{kDa}$ and $100 \mathrm{kDa}$

$\mathrm{HA}$ at various ratios after $18 \mathrm{~h}$ incubation

5.4 Plot of fluorescence intensities of CPN vs incubation time at optimized formulation of $\mathrm{CPN} / \mathrm{HA}$ showing six-fold enhancement of CPN-PEG $2000-\mathrm{FA}$ uptake and three-fold enhancement of CPN-PEG 2000

5.5 UV-absorption and emission spectra for $\mathrm{CPN}^{-P_{E G}}{ }_{2000}-\mathrm{FA}$ and $\mathrm{CPN}-\mathrm{PEG}_{2000}$ complexed with $40 \mathrm{kDa} \mathrm{HA}$ at $1: 1$ and 1:2 molar ratios

5.6 Mean sizes and size distributions as measured by NTA of CPN/HA complexes.

5.7 Effect of endocytosis inhibitors of CPN-PEG $2000-F A$ and CPN-PEG 2000

5.8 High-resolution fluorescence microscopic images of blue and red channel overlay, and contrast images of cellular uptake in HeLa cells for CPN-PEG $2000-F A, C{ }_{2}-P_{2} G_{2000}-F A / H A$, CPN-PEG 2000 , and CPN-PEG $2000 / \mathrm{HA}$

S5.1 ${ }^{1} \mathrm{H}$ NMR spectra of PPE1 and CPN-PEG $2000-F A$ in DMSO- $\mathrm{d}_{6}$

S5.2 UV-vis absorption spectra of folic acid in the supernatant showing complete removal of folic acid from CPN PEG 2000 -FA solution via dialysis

S5.3 FT-IR spectra of PPE1 and CPN-PEG 2000 -FA......................................................................132

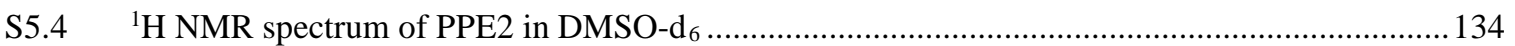

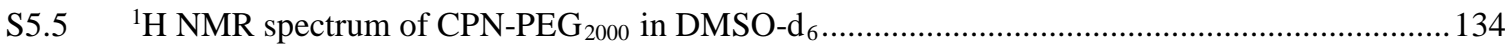

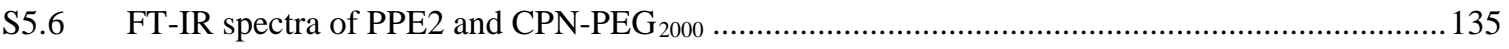

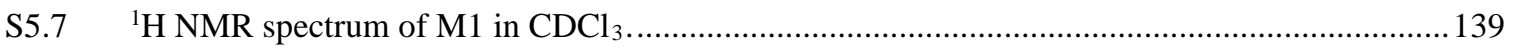

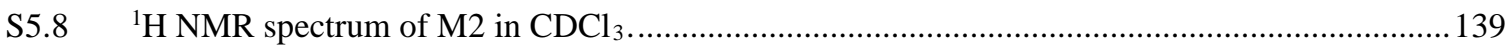

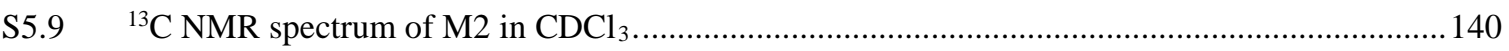

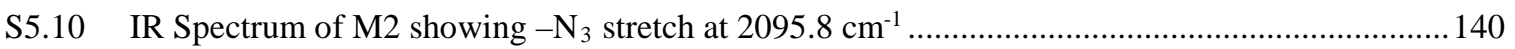

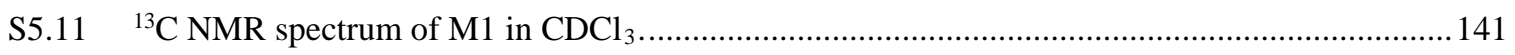

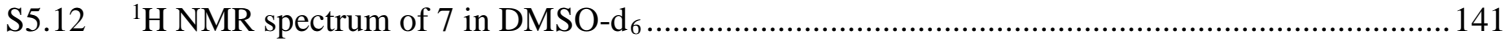

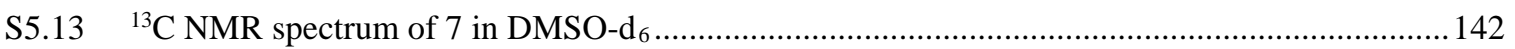

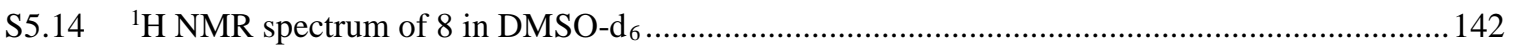

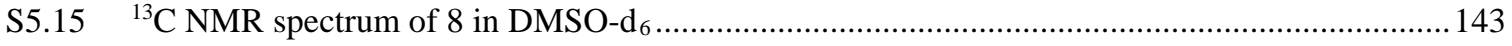

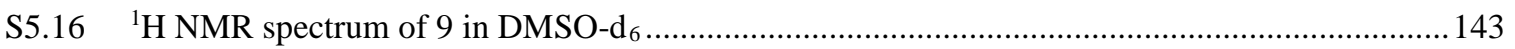

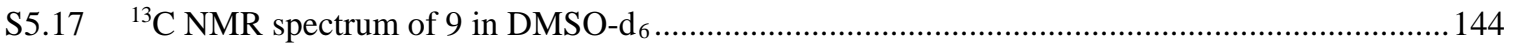




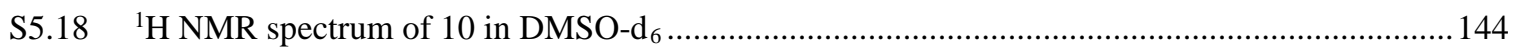

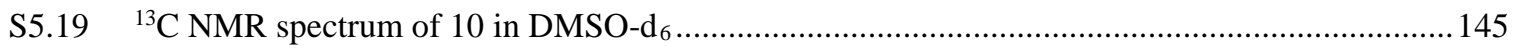

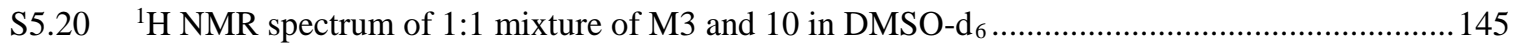

S5.21 Absorption and emission spectra for PPE2 in THF .......................................................... 146

S5.22 Zeta potential for CPN-PEG $2000-F A(a)$ and CPN-PEG 2000 (b). ......................................... 146

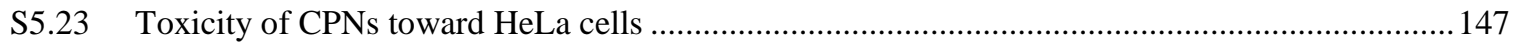

S5.24 Quenching experiment of CPN-PEG $2000-F A$ and CPN-PEG 2000 with trypan blue .....................147

S5.25 Representative scatterplots and intensity plots for optimized conditions for

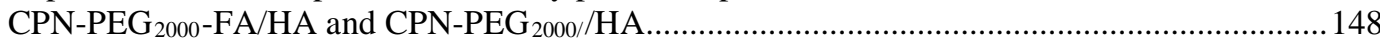




\section{LIST OF SCHEMES}

SCHEME

PAGE

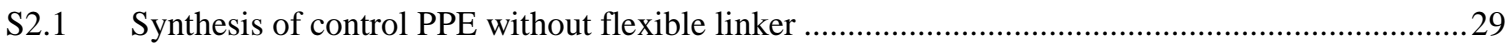

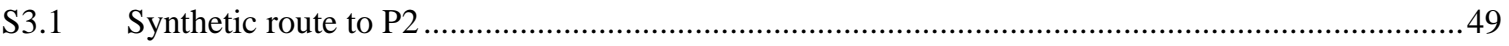

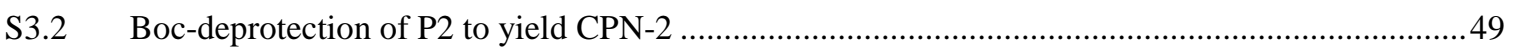

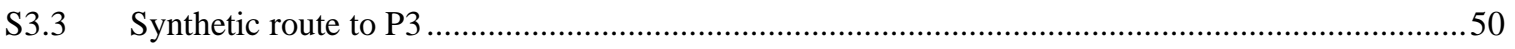

S3.4 Boc-deprotection of P3 to yield CPN-3 …….............................................................................

4.1 TPP (R1)-containing polymers with (PPE-L) and without (PPE) biodegradable linker and

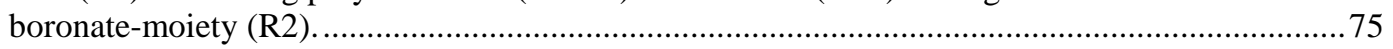

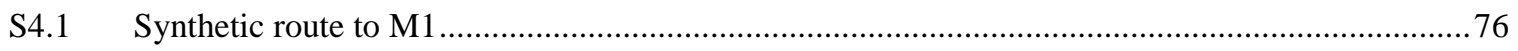

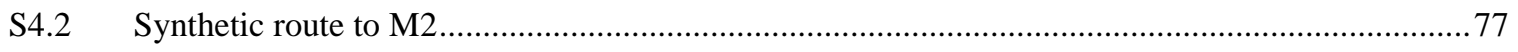

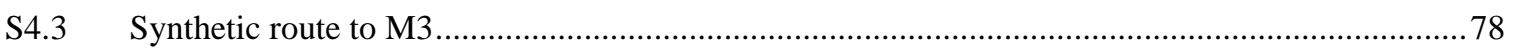

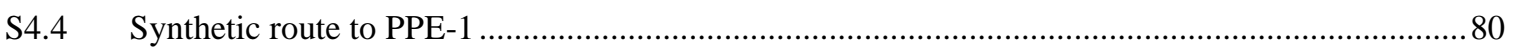

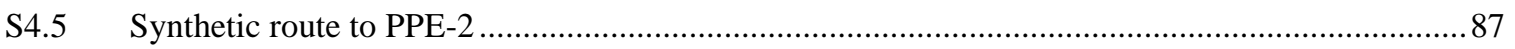

S5.1 Synthesis of monomers (M1, M2) and heterodifunctional PEG2000 (M3) ..................................124

S5.2 Synthesis of water-soluble PPE1 from readily available monomers via Sonogashira coupling

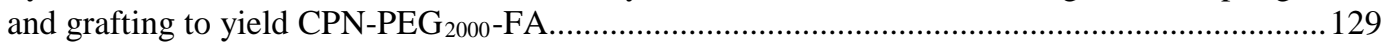

S5.3 Click reaction of alkyne end-functionalized PEG 2000 onto azide end-functionalized PPE2 to yield CPN-PEG 2000 . 


\section{ABBREVIATIONS AND ACRONYMS}

\begin{tabular}{|c|c|}
\hline AA & acetic acid \\
\hline AFM & atomic force microscopy \\
\hline Boc & $t$-butyl carbamate \\
\hline $\mathrm{BTz}$ & benzothiadiazole \\
\hline CD44 & Cluster Determinant 44 receptor \\
\hline CMC & carboxymethyl cellulose \\
\hline CME & clathrin-mediated endocytosis \\
\hline CvME & caveolae-mediated endocytosis \\
\hline $\mathrm{CP}$ & conjugated polymer \\
\hline CPE & conjugated polymer electrolyte \\
\hline $\mathrm{CPN}$ & conjugated polymer nanoparticle \\
\hline CS & chondroitin sulfate \\
\hline DCM & dichloromethane \\
\hline DIPA & $N, N$-diisopropylamine \\
\hline DLS & dynamic light scattering \\
\hline dppf & 1,1'-bis(diphenylphosphino)ferrocene \\
\hline DMF & dimethylformamide \\
\hline DMSO & dimethylsulfoxide \\
\hline DS & dermatan sulfate \\
\hline EtOH & ethanol \\
\hline EPR & enhanced permeability and retention \\
\hline EO & ethylene oxide \\
\hline FDA & Food and Drug Administration \\
\hline FA & folic acid \\
\hline FR & folate receptor \\
\hline FRET & fluorescence resonance energy transfer \\
\hline
\end{tabular}




\begin{tabular}{|c|c|}
\hline FT-IR & Fourier transform infrared spectroscopy \\
\hline GPC & gel permeation chromatography \\
\hline GSH & glutathione \\
\hline HA & hyaluronic acid \\
\hline HBT & homo-coupled benzothiadiazole \\
\hline HEK & human embryonic kidney \\
\hline HeLa & human cervical carcinoma \\
\hline HS & heparin sulfate \\
\hline HOMO & highest occupied molecular orbital \\
\hline HR-MS & high-resolution mass spectrometry \\
\hline ISC & intersystem crossing \\
\hline${ }^{\mathrm{i}} \mathrm{PrOH}$ & isopropyl alcohol \\
\hline LDA & linear discriminant analysis \\
\hline LUMO & lowest unoccupied molecular orbital \\
\hline $\mathrm{MeCN}$ & acetonitrile \\
\hline $\mathrm{MeOH}$ & methanol \\
\hline $\mathrm{M}_{\mathrm{n}}$ & number average molecular weight \\
\hline MPC & macropinocytosis \\
\hline $\mathrm{M}_{\mathrm{w}}$ & weight average molecular weight \\
\hline $\mathrm{m} / \mathrm{z}$ & mass-to-charge ratio \\
\hline NMR & nuclear magnetic resonance \\
\hline NP & nanoparticle \\
\hline NTA & nanoparticle tracking analysis \\
\hline OD & optical density \\
\hline PA & poly(acetylene) \\
\hline PCC & Pearson's correlation coefficient \\
\hline PEDOT & polyethylene-doxythiophene \\
\hline
\end{tabular}




\begin{tabular}{|c|c|}
\hline PEG & polyethylene glycol \\
\hline PDI & polydispersity index \\
\hline $\mathrm{Pd}\left[\mathrm{Cl}_{2}\left(\mathrm{PPh}_{3}\right)_{2}\right]$ & bis(triphenylphosphine)palladium(II) dichloride \\
\hline $\mathrm{Pd}(\mathrm{OAc})_{2}$ & palladium(II) acetate \\
\hline $\mathrm{Pd}\left[\left(\mathrm{PPh}_{3}\right)_{4}\right]$ & tetrakis(triphenylphosphine)palladium(0) \\
\hline РРВ & poly(p-phenylenebutadiynylene) \\
\hline PPE & poly(p-phenyleneethynylene) \\
\hline PPP & poly(p-phenylene) \\
\hline PPV & poly(p-phenylenevinylene) \\
\hline PPy & polypyrrole \\
\hline PT & poly(thiophene) \\
\hline PTFE & polytetrafluoroethylene \\
\hline QY & quantum yield \\
\hline $\mathrm{R}$ & substituent \\
\hline RES & reticuloendothelial system \\
\hline RHAMM & receptor for HA mediated motility \\
\hline SEC & size exclusion chromatography \\
\hline $\mathrm{rt}$ & room temperature \\
\hline TEA & triethylamine \\
\hline TFA & trifluoroacetic acid \\
\hline THF & tetrahydrofuran \\
\hline ТPP & triphenylphosphonium \\
\hline UV-Vis & ultraviolet-visible \\
\hline
\end{tabular}


CHAPTER I

Introduction 


\subsection{Materials chemistry}

Synthetic polymeric materials have gained significant use as biological materials (biomaterials) in biomedical and pharmaceutical applications. As a result, a demand for well-defined polymers with tunable properties has emerged. The method of system's materials chemistry utilizes the investigation of the interactions between components of a system, to understand how those interactions drive the function and performance of the overall system. ${ }^{1}$ The development of functional materials is built on rational design, synthesis/fabrication, and systematic characterization of properties. In order to design a material for a desired application successfully, an in-depth understanding of the structure-function relationship is crucial. This understanding allows the development of a material that possesses the necessary and desired properties. More importantly, an understanding of how those properties relate to a material's chemical and physical structures. The systematic approach to material design is essential as it is no longer effective to isolate only one component to solve complex scientific challenges. As advances in effective material development occur, it is increasingly evident that these advances are only achieved when an integrated approach is employed.

\subsection{Challenges for polymeric biomaterials}

Biomaterials are defined as substances other than food or drugs used for therapeutic and diagnostic applications that can be composed of both natural and synthetic components. ${ }^{2}$ Biomaterials are usually polymeric to address the biological, physical, and chemical demands of their applications. These biomaterials have been at the forefront of treating diseases and improving healthcare, and are most often biologically derived or inspired molecules. Modern biomaterials have been used as synthetic and natural replacements for biological tissues, materials for medical device implantation, carriers for drug delivery systems, and materials for array-based sensing for diagnostic applications. ${ }^{2}$ However, these materials still suffer from issues with biocompatibility, mechanical properties, and proper degradation. There are several specific biological challenges that need to be addressed to further biomaterial development (discussed in additional in section 1.9), including targeting materials for drug delivery to specific cells and organelles, materials for sensing various biochemical signals, and strategies for improved biocompatibility of materials. Synthetic materials, such as polymers, offer a simple and effective approach to address these issues. The synthetic versatility of 
polymeric materials allows an opportunity to understand the structure-property relationship of materials and their cellular interactions.

\subsection{Conjugated polymers}

A novel class of polymeric biomaterials are conjugated polymers (CPs), which are polyunsaturated and consist of a backbone chain of alternating single and double (or triple) bonds. ${ }^{10}$ Examples of CPs are depicted in Figure 1.1. The semi-conductive properties of CPs are the result of the electronic conjugation of delocalized pi $(\pi)$ electrons (generalized example shown in Figure 1.2) along the backbone, which provides these compounds with desirable physiochemical and excellent photophysical properties, including inherent fluorescence. ${ }^{11}$ The energy band gap is defined by the highest occupied molecular orbital (HOMO) and lowest unoccupied molecular orbital (LUMO) formed from the sp and $\mathrm{sp}^{2}$ hybridized carbon atoms along the conjugated backbone and is the origin of the photophysical and semi-conductive properties. ${ }^{4}$<smiles>CC(C)(C)/C=C/C(C)(C)C</smiles>

polyacetylene

(PA)

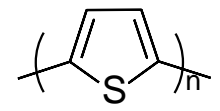

polythiophene

(PT)

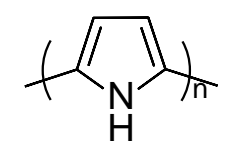

polypyrrole

(PPy)

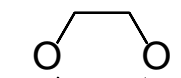

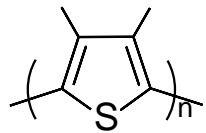

polyethylenedioxythiophene (PEDOT)

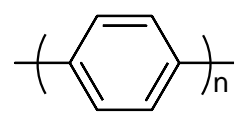

poly(para-phenylene) (PPP)

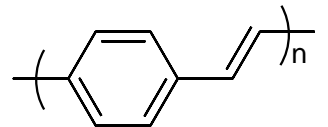

poly(para-phenylenevinylene) (PPV)

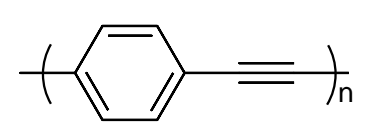

poly(para-phenyleneethynylene)

(PPE)

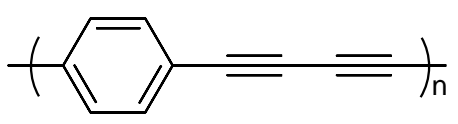

poly(para-phenylbutadiynylene) (PPB)

Figure 1.1. Common examples of conjugated polymers. 


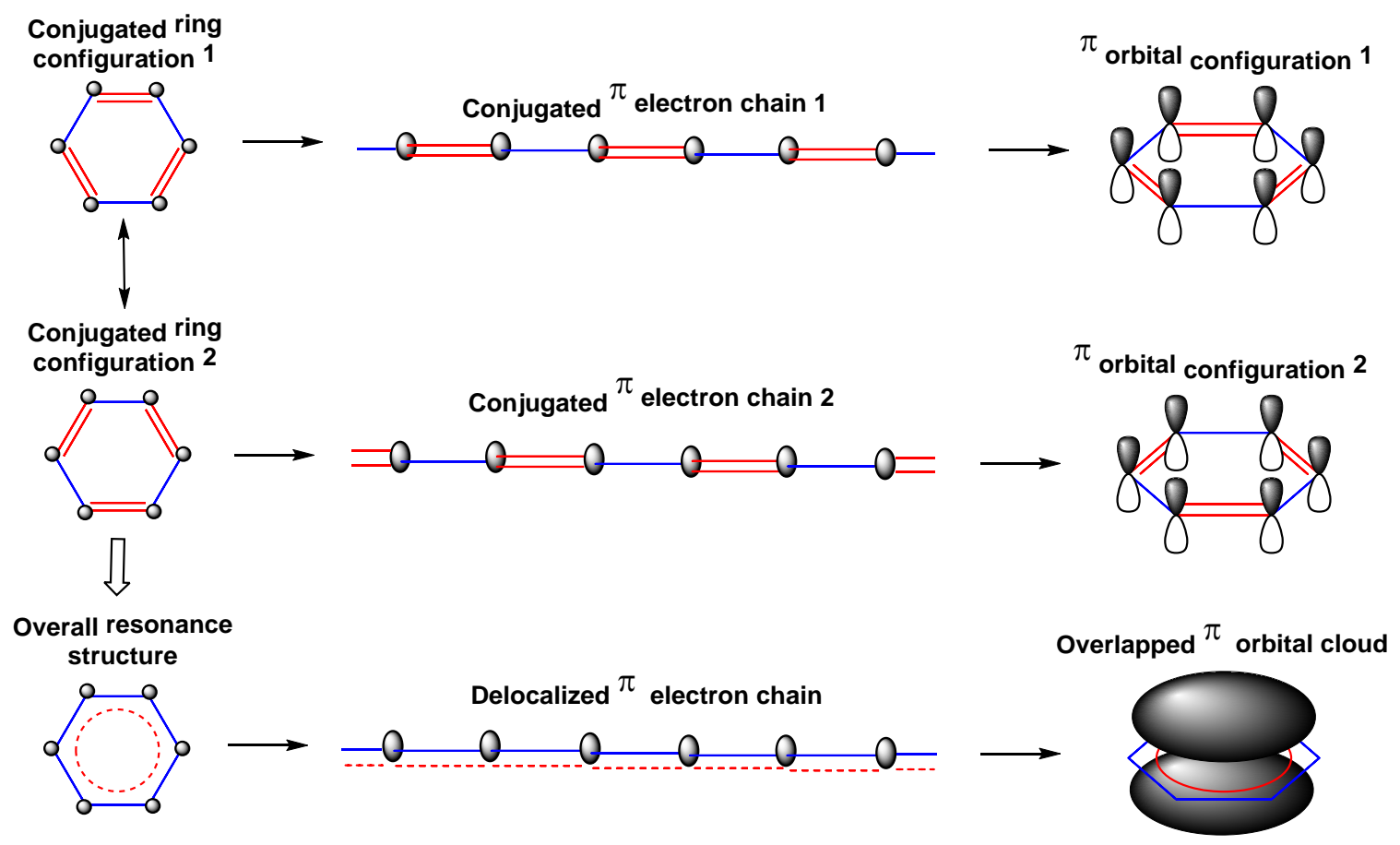

Figure 1.2. Example configuration of delocalized electrons of a $\pi$ conjugated system.

Recently, CPs have been applied to several electronic and sensing applications, including solar cells, lightemitting diodes, and a variety of chemical and biological sensors. ${ }^{12-16}$ While these applications encompass a wide variety of CPs, the Moon research group focuses on poly(para-phenyleneethynylene) (PPE) and poly(para-phenylbutadiynylene) (PPB)-based polymers, because of their ease of synthesis, for biological applications including cellular imaging, cancer cell targeting and labelling, and sensing of biologically important substances. The synthetic versatility of CPs allow easy modification of the conjugated backbone to tune emission and side chain structures to adjust biocompatibility through increased water solubility, controlled biodegradability, and incorporation of targeting units.

\subsubsection{Synthesis}

The backbone and side chain structures of CPs gives rise to unique properties. The conjugated backbone provides a structural platform for tuning the electronic properties, and side chain structure can be used to modulate hydrophobicity/hydrophilicity and provide functionalization. The synthesis of PPEs and PPBs utilizes the cross-coupling reactions of Sonogashira and Glaser, respectively. The Sonogashira reaction uses a palladium catalyst, with a catalytic amount of copper and base, to couple an aryl (or vinyl) halide to a 
terminal alkyne, and has been shown to be very versatile. ${ }^{10}$ Glaser coupling is used for homocoupling of terminal acetylenes in the presence of a copper catalyst under an oxygen-rich environment. Glaser coupling also occurs with the Sonogashira reaction and results in a homocoupled side product when nonoxidative conditions are not maintained. Generalized reactions for PPE and PPB synthesis using Sonogashira and Glaser coupling, respectively, are shown in Figure 1.3.
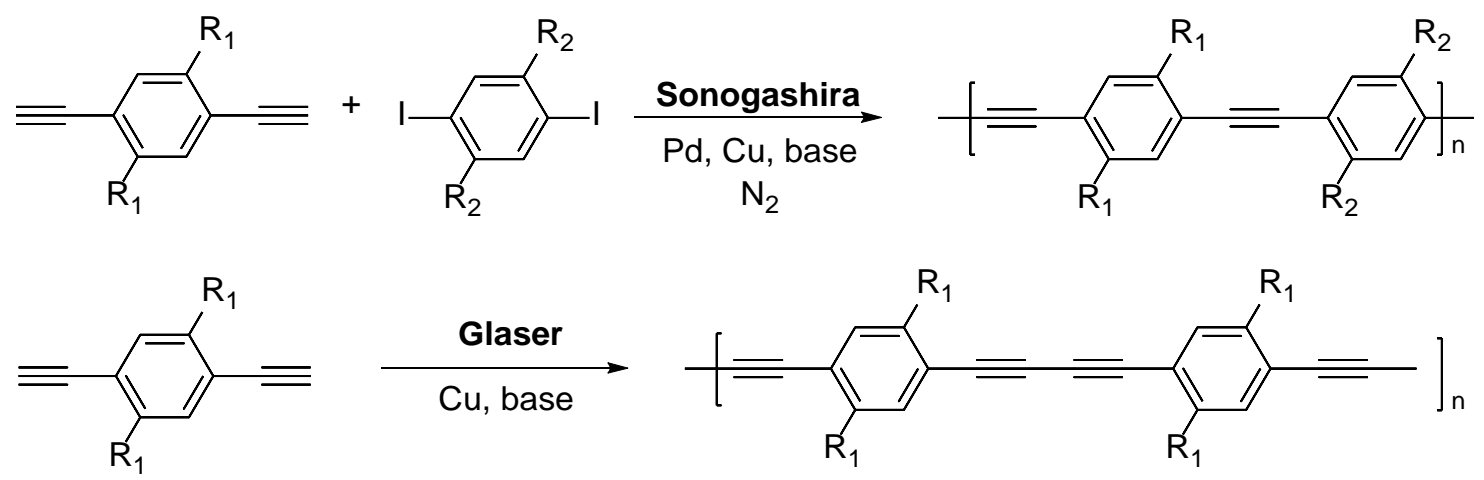

Figure 1.3. Sonogashira and Glaser coupling for PPE and PPB synthesis, respectively.

\subsubsection{Photophysics}

In an ideal environment (i.e., organic solvent, contaminant-free), CP chains are well-solvated and exhibit photophysical properties originating from the delocalized electrons along the conjugated backbone. ${ }^{2}$ These delocalized $\pi$ electrons (depicted in Figure 1.2) are responsible for the characteristic absorption of light and subsequent relaxation from excited electronic states via fluorescence. ${ }^{3}$ The intrinsic fluorescence makes CPs an advantageous choice over other macromolecules, specifically for sensing and imaging applications. The semi-conductive and photophysical properties of CPs are driven by the HOMO-LUMO gap, which is also responsible for the wavelength of absorbed and emitted light. These processes can be described by a Jablonski diagram (Figure 1.4), which represents the energy states of a molecule's electrons and the transitions between states. ${ }^{5}$ When a molecule absorbs light, the electron in the ground singlet state $\left(\mathrm{S}_{0}\right)$ is excited to a vibrational level in the excited singlet state $\left(\mathrm{S}_{1}\right)$, in a rapid process $\left(\sim 10^{-15} \mathrm{~s}\right)$. The excess in energy can be released in several ways to return back to the ground state. Through internal conversion via non-radiative decay, the excited electron relaxes to the lowest vibration level of the excited singlet state $\left(\mathrm{S}_{1}\right)$. In fluorescence, the excited electron returns to the ground state $\left(\mathrm{S}_{0}\right)$ by emitting a photon, which is a fast $\left(\sim 10^{-9} \mathrm{~s}\right)$ and favorable process, and responsible for the desirable photophysical properties of CPs. An alternative pathway for an 
excited electron to return to the ground state is via phosphorescence, where the electron undergoes intersystem crossing (ISC) and spin-forbidden electronic transitions in a much slower process $\left(\sim 10^{-3} \mathrm{~s}\right)$. The emission efficiency, or fluorescence brightness, is quantified using the quantum yield, which is the ratio of photons emitted to photons absorbed. The brightness of new fluorescent materials is determined relative to a known bright standard.
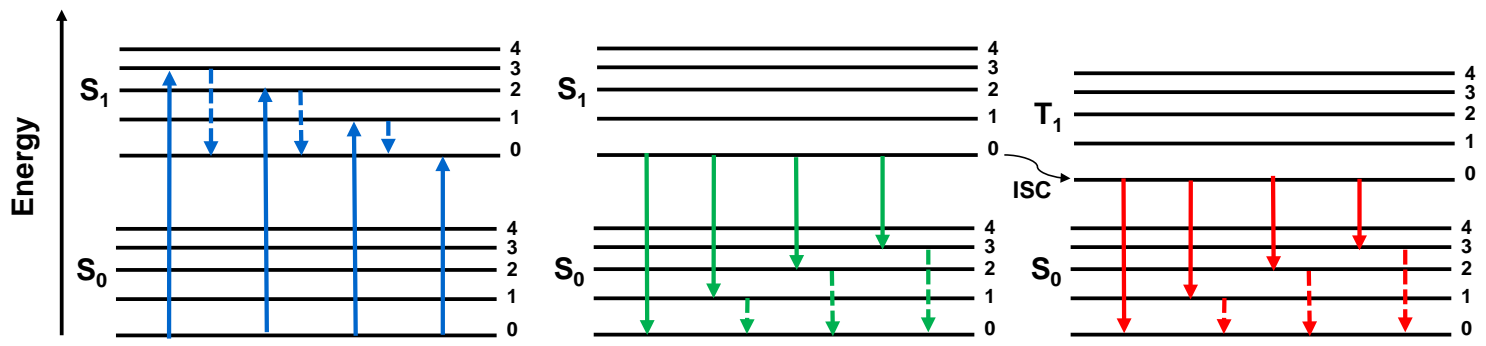

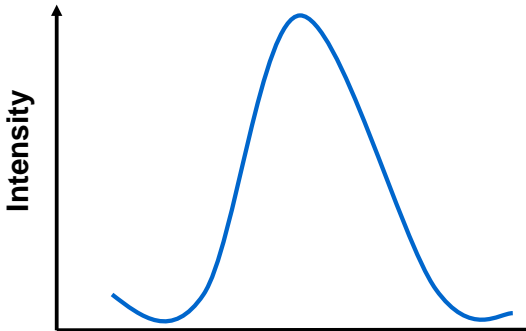

Absorption

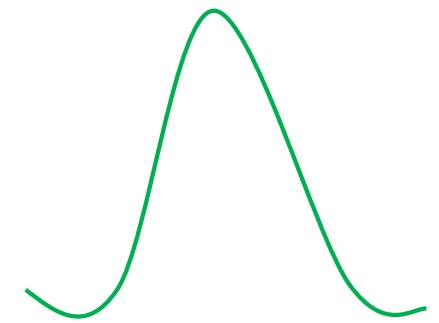

Fluorescence

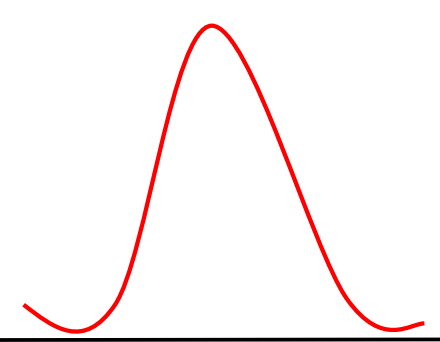

Phosphorescence

Figure 1.4. Jablonski diagram showing the various processes when a molecule is irradiated by light.

\section{$1.4 \quad$ Conjugated polymer nanoparticles}

For biological applications, CPs must be compatible with aqueous environments. Because of the hydrophobic nature of the conjugated backbone, ionic and hydrophilic side chains can be incorporated to achieve the necessary aqueous solubility. When a CP dissolved in an organic solvent is added to an excess amount of aqueous solvent, the hydrophobic CP chains aggregate and form nanoparticles. These CP nanoparticles (i.e., CPNs) are then purified via dialysis to remove the organic solvent. This type of nanoparticle (NP) preparation is termed the reprecipitation method, and the resulting NPs are in the range of 5-150 nanometers (nm) in diameter. ${ }^{6}$ The process of how CP chains aggregate and self-assemble in an aqueous environment is driven by the hydrophobic backbone where both inter-chain aggregation and intra-chain collapse can occur. While the hydrophobic effect is the main driving force of NP formation, the nature of polymer chain self-assembly can be modulated by the side chain structure, where ionic groups can cause repulsion between chains and 
increased hydrophilicity can improve solubility. Recently, Ko et al. demonstrated controlled chain-chain interactions of CPNs fabricated from primary amine-containing PPEs as exhibited by different aggregation structures and photophysical properties determined by organic acid treatment of the CPs. ${ }^{28}$ The different aggregation structures were the result of minimal $\pi-\pi$ stacking in loosely aggregated CPNs treated with mild acetic acid (AA) and extensive $\pi-\pi$ stacking in densely aggregated CPNs treated with dicarboxylic tartaric acid (TA). The resulting aggregation state of CPNs is, therefore, a cooperative assembly determined by the $\pi-\pi$ stacking, hydrogen bonding, and the interplay of hydrophobic, van der Waals, and electrostatic forces. ${ }^{8}$ In addition, NP formation can be adjusted on the basis of polymer stock concentration and molecular weight/distribution of the polymer. ${ }^{7}$

\subsection{Polymer characterization}

To understand the structure-function relationship for a material and its application, it is necessary to characterize the structure properly, which can be achieved using a variety of techniques. After synthesis and purification, the polymer's molecular weight is determined by gel permeation chromatography (GPC), which is a type of size exclusion chromatography (SEC) and the most convenient method for use with polymers. ${ }^{9}$ The technique of GPC is a physical chromatographic separation determined by size, where larger molecules pass through the porous column more rapidly than smaller molecules that elute slowly as they are retained within the pores of the column. The molecular weight distribution is determined relative to standards (i.e., polystyrene or polyethyleneglycol) and described by the weight average molecular weight $\left(\mathrm{M}_{\mathrm{w}}\right)$, number average molecular weight $\left(\mathrm{M}_{\mathrm{n}}\right)$, and polydispersity index (PDI). An example GPC chromatogram is depicted in Figure 1.5. 


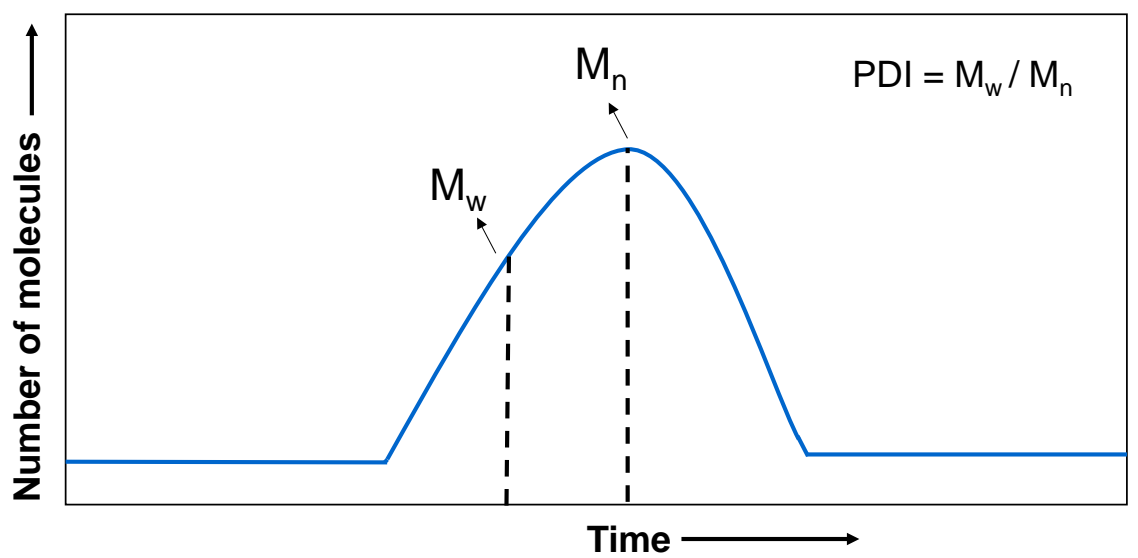

Figure 1.5. Example GPC chromatogram illustrating molecular weight distribution.

The chemical structure of a polymer can be ascertained quantitatively using nuclear magnetic resonance (NMR) spectroscopy, which is an analytical structure determination method. The basic principles of NMR rely on the changes in magnetic moments of magnetically active nuclei, such as ${ }^{1} \mathrm{H},{ }^{13} \mathrm{C},{ }^{19} \mathrm{~F}$, and ${ }^{31} \mathrm{P}$, when exposed to a radio frequency and a strong external magnetic field. The energy that is emitted upon relaxation of the excited nuclei is characteristic to the nuclei themselves and the chemical environment they are exposed to within the molecule. The NMR spectrum provides information about the proportion of nuclei generating the signal given by the integrated area under each peak, and the nature of neighboring nuclei given by the splitting pattern of the peak. The technique of NMR is a very powerful structural elucidation technique, however, it provides structural information for the average polymer rather than exact structures of individual polymer chains.

The presence of certain functional groups in a polymer can be determined using Fourier Transform-Infrared (FT-IR) spectroscopy, which is a qualitative technique that provides structural information about the bonds between atoms within a molecule. When IR radiation is passed through a sample, it is absorbed and transmitted on the basis of frequencies of vibrations between the bonds of atoms present in the sample. The absorption region is characteristic of a functional group as the radiation is characteristic of that bond and can be used to confirm the presence or absence of certain groups in monomer and polymer samples.

\subsection{Polymer self-assembly characterization}

While chemical structure characterization is critical for developing new materials, it is crucial to understand how that material exists in solution and, eventually upon interaction with biological interfaces. This will 
ultimately determine how well the material will perform its function and provide information to understand structure-property relationship. The absorption and emission profiles of PPE- and PPB-containg CPNs are characteristic of the electronic states of the polymer structure (discussed in section 1.5), which are generally broad compared to small molecules, and more defined compared to PPV polymers due to the rigidity of the conjugated backbone. The absorption and emission wavelength maxima are characteristic of the type of conjugated backbone and conjugation length, this is the result of the configuration of the delocalized $\pi$ electron cloud (discussed in section 1.3). As conjugation length increases, the HOMO-LUMO gap decreases and results in a bathochromic shift (towards red) of the wavelength maxima.

The effect of $\pi-\pi$ stacking between polymer chains also drives the wavelength maxima and shape of absorption and emission profiles. The CP chains can be compared to that of small organic dyes that exhibit specific superstructures, $\mathrm{J}$ and $\mathrm{H}$ aggregates, as shown in Figure 1.6. When the delocalized $\pi$ electron cloud of neighboring polymer chains align face-to-face, there is a hypsochromic shift (towards blue) in wavelength maxima due to an increase in energy between the HOMO and LUMO, this is called an H aggregate. ${ }^{35}$ In comparison to when neighboring polymer chains align end-to-end, there is a bathochromic shift because of a decrease of the HOMO-LUMO gap, this is called a J aggregate. ${ }^{35}$ Red shifting in wavelength maxima also occurs when there is strong $\pi-\pi$ stacking between polymer chains, which causes an increase in effective conjugation length that decreases the HOMO-LUMO gap. Absorption and emission spectra are indicative of polymer self-assembly, and used to observe and understand the changes in polymer aggregation when external stimuli are applied (i.e., solvent, $\mathrm{pH}$, salt concentration, or polyanions). 


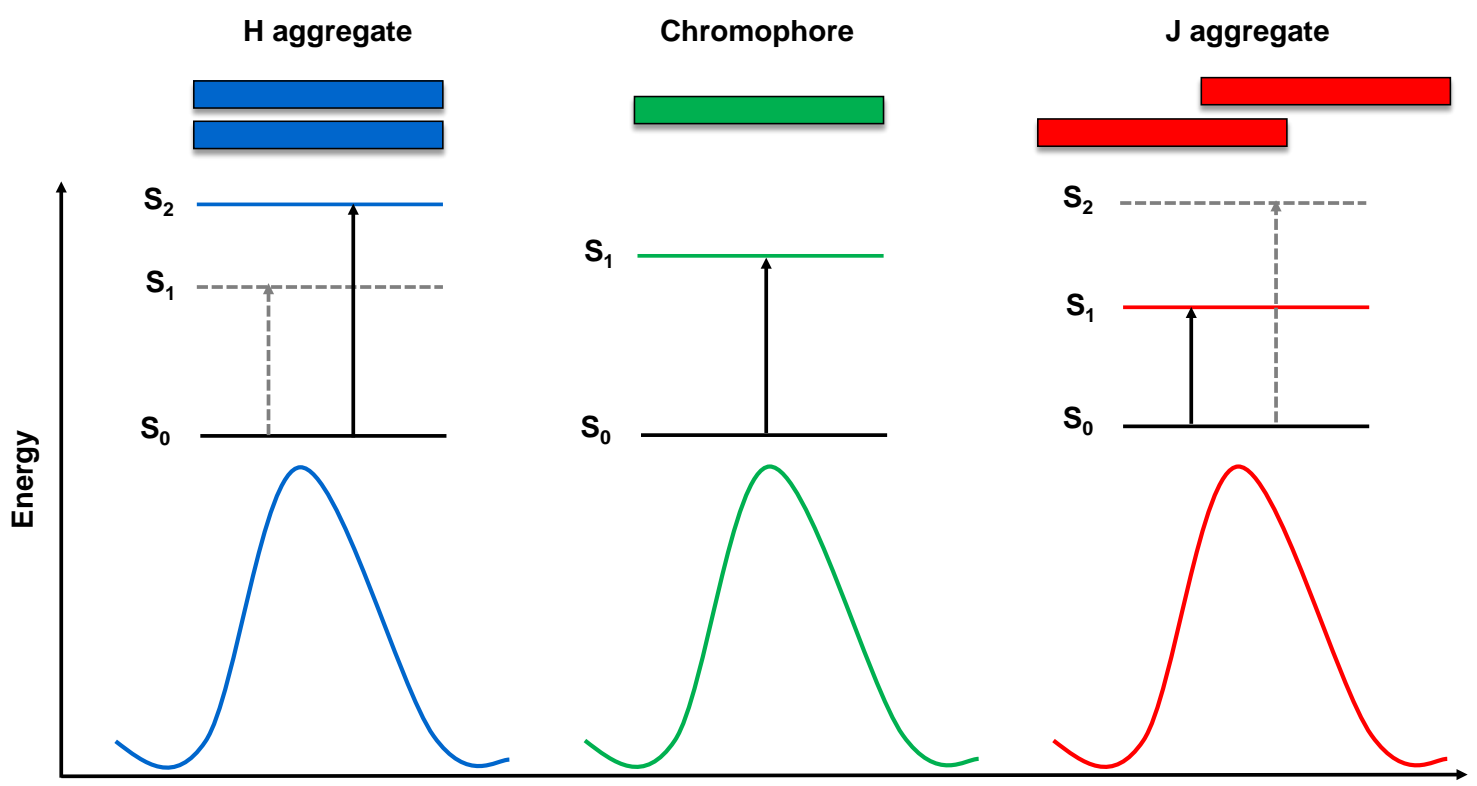

Wavelength

Figure 1.6. Schematic representation of the spectral shift in $\mathrm{H}$ and J-aggregates based on chromophore arrangement.

Absorption of light is not only characteristic of a material's electronic states, but also the concentration of absorbing molecules in solution. The absorbance (A) of a solution is defined as the logarithm ratio of incident light $\left(\mathrm{I}_{0}\right)$ to transmitted light $(\mathrm{I})$ and is described by the Beer-Lambert law:

$$
A=\log _{10} \frac{I_{0}}{I}=\varepsilon l c
$$

where $\varepsilon$ is the molar absorptivity (i.e.; an intrinsic property of the absorbing material) in $\mathrm{mol}^{-1} \cdot \mathrm{L}^{\cdot} \mathrm{cm}^{-1}, l$ is the path length of the absorbing solution in centimeters $(\mathrm{cm})$, and $\mathrm{c}$ is the concentration of absorbing material in $\mathrm{mol} \cdot \mathrm{L}^{-1}$.

In addition to characterizing the structural and photophysical properties of polymer self-assembly, it is important to characterize the physical properties of the polymer nanoparticle. These properties will also provide insight into understanding the material’s function and performance. Dynamic light scattering (DLS), also called photon correlation spectroscopy, is a technique used to determine particle size by measuring the intensity of light scattered by particles in suspension. Light is scattered after the interaction with the electric field of a particle, which causes an incident photon to induce an oscillating dipole in the electron cloud and radiation is dispersed in all directions as the dipole changes. The fundamental theory of DLS is built upon 
the Brownian motion of spherical particles, which describes the random movements of particles because of collisions caused by the solvent that surrounds those particles. The scattered light is recorded over time and the correlation function is used to determine the mean size and polydispersity index, which is an estimate of the distribution width. DLS relates the speed of the particles in terms of the diffusion constant to the particle size as expressed by the Stoke-Einstein equation:

$$
\mathrm{r}_{\mathrm{h}}=\frac{\mathrm{kT}}{6 \pi \eta \mathrm{D}}
$$

where $\mathrm{r}_{\mathrm{h}}$ is the hydrodynamic radius, $\mathrm{k}$ is the Boltzmann constant, $\mathrm{T}$ is temperature in Kelvin, $\eta$ is the solvent viscosity, and D is the diffusion coefficient. The particle size, termed the hydrodynamic radius, is defined as the radius of a hard sphere that diffuses at the same speed as the particle measured and is dependent on the size of the particle core and the surface structure of the particle, including any ions in solution. Surface structure is an important factor since the particle diffusion speed will be changed on the basis of the thickness of the electric double layer of the particle.

The zeta potential (illustrated in Figure 1.7) is measured using electrophoresis, where the movement of a charged particle relative to the medium under an applied electric field is recorded. The particle moves towards the electrode of opposite charge and is opposed by viscous forces acting on the particle. When an equilibrium is reached between these two forces, the particle moves with a constant velocity that is dependent on the electric field strength, dielectric constant of the medium, viscosity of the medium, and zeta potential. 


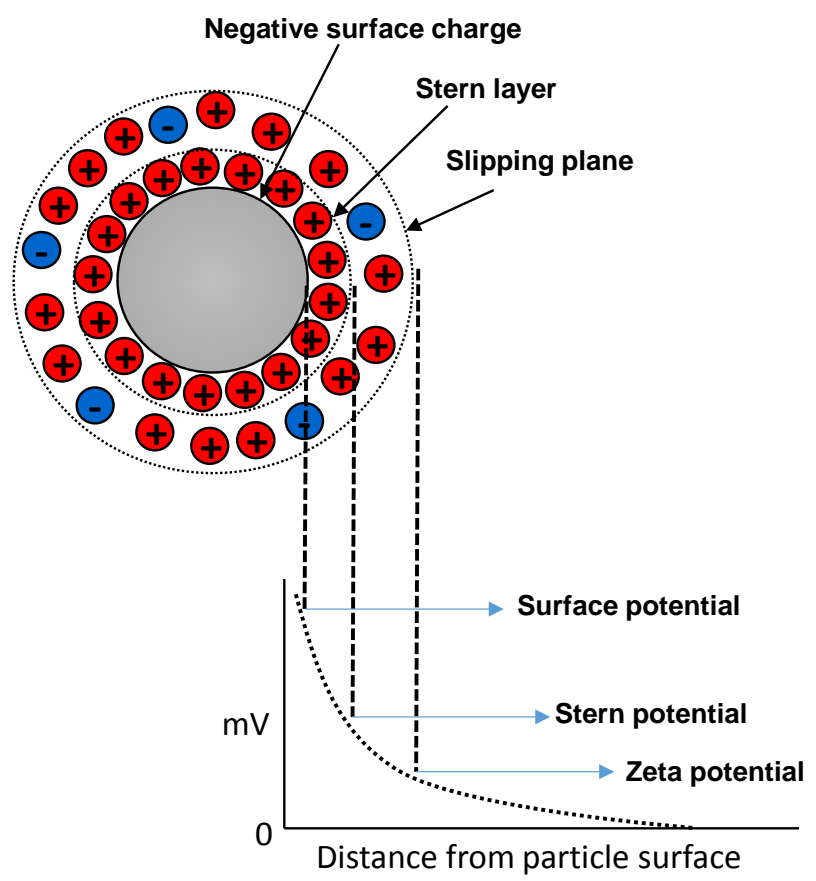

Figure 1.7. Schematic representation of zeta potential of a negatively charged particle.

The velocity of the particle in an electric field is termed electrophoretic mobility and is related to the zeta potential using the Henry equation:

$$
\mathrm{U}_{\mathrm{E}}=\frac{2 \varepsilon \mathrm{Zf}(\kappa \mathrm{a})}{3 \eta}
$$

where $U_{E}$ is the electrophoretic mobility, $\varepsilon$ is the dielectric constant of the medium, $Z$ is the zeta potential, $\eta$ is the viscosity of the medium, and $\mathrm{f}(\kappa \mathrm{a})$ is the Henry's function. For nano-sized particles in solution, or colloids, the stability is dependent on the repulsive forces between the particles. Repulsion can be achieved via steric repulsion, where adsorbed bulky groups prevent particles from coming into contact with each other, and electrostatic stabilization, where particles are distributed on the basis of their like charges. In aqueous suspensions, most colloids carry an electric charge, which can be the result of ionized surface groups, differential loss of ions from the crystal lattice structure, and adsorbed charges species. The magnitude of the zeta potential is a direct indication of particle stability; greater than $+/-30 \mathrm{mV}$ is considered stable, and indicative of the particle's surface charge. The zeta potential provides important information about what is happening at the interface of the material's surface and its solution. 
While DLS is typically used to measure the bulk composition of particles in solution, it is only accurate for monodispersed systems. Larger particles will scatter more light than smaller ones, and therefore, contribute more to the overall size measurement. An emerging technique for analyzing single particles in a polydispersed system is nanoparticle tracking analysis (NTA). A laser beam is passed through the sample and the scattered light is captured by a charge-coupled device (CCD) camera mounted onto a microscope that records a video at 30 frames per second. ${ }^{17}$ The software determines the displacement of individual particles by tracking the $\mathrm{x}$ and $\mathrm{y}$ movement of each particle's center, frame-by-frame. Once the displacement is calculated, the diffusion coefficient and size are determined using the Stokes-Einstein equation (Eq. 1.1). The technique of NTA is more favorable for polydispersed samples, like CPNs, and is capable of determining size at much lower concentrations than DLS. However, DLS is a user friendly technique, with a short analysis time, and provides reliable and consistent data since it measures the average sample.

\subsection{Biological use of nanoparticles}

Nanotechnology has made tremendous advancements for diagnostics, therapeutics, and imaging. The use of nanoparticles offers several advantages over traditional delivery methods (i.e., free drugs) including encapsulation, enhanced retention, controlled release, and reduced toxicity to off-target sites. In particular, typical chemotherapeutics have a narrow therapeutic because of poor solubility, limited circulation time, and cytotoxicity to healthy tissue. By using water-soluble polymers, nanoparticle-based drug delivery systems can enhance aqueous solubility of therapeutics. Nanoparticles can be used to increase half-life and address the pharmacokinetics issues to improve therapeutic effects. The tunability of nanoparticle structure is an excellent platform to improve target-ability, in both reducing off-target effects, and enhanced retention and accumulation in diseased tissue. Several nanocarrier therapeutics with a variety of formulations have been approved by the Food and Drug Administration (FDA) for clinical trials and use, including inorganic nanoparticles, ${ }^{20}$ liposomes, micelles, ${ }^{21}$ protein nanoparticles, ${ }^{22}$ polymeric micelles, ${ }^{23}$ polymer-drug

conjugates, ${ }^{24}$ and polymer nanoparticles. ${ }^{25}$ Nano-sized systems have a favorable small size that leads to passive targeting and preferential delivery to diseased tissue because of the enhanced permeability and retention (EPR) effect, as illustrated in Figure 1.8. The EPR effect arises as a consequence of the nature of 
tumor tissue, which is hypervascular, has up regulation of proangiogenic signaling, and poor lymphatic drainage that allows for accumulation of large molecules $(\geq 40 \mathrm{kDa})$ in the microenvironment. ${ }^{19}$ Additionally, the small size has reduced side effects as nanoparticles escape recognition from the reticuloendothelial system (RES) in healthy tissue. ${ }^{30}$

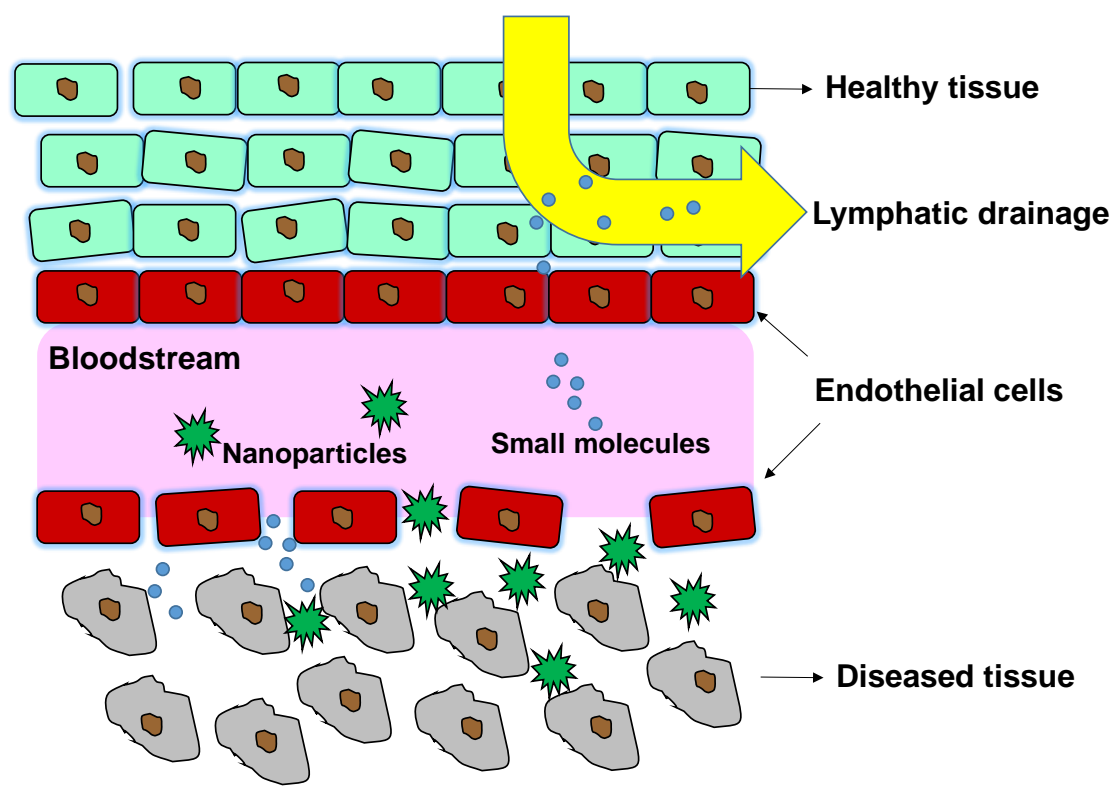

Figure 1.8. Schematic representation of the enhanced retention and permeability (EPR) effect that occurs in diseased tissue. Adapted from Stockhofe et al. ${ }^{36}$

\subsubsection{Cellular uptake of nanoparticles}

To develop materials for biological use, there are several barriers that must be traversed to be effective. The first is the physiological barrier, where nanomaterials have been shown to easily traverse as a consequence of their size. The second important barrier is the cellular barrier and in particular, the cell membrane. The cellular membrane is responsible for the selective flow of ions and molecules into and out of the cell. Most importantly, the cell membrane is responsible for the maintenance of separation between the cytosol and extracellular environment. ${ }^{18}$ Extracellular molecules are selectively transported into the cell via endocytosis pathways: phagocytosis (i.e., “cell-eating”), pinocytosis (i.e., “cell-drinking”), macropinocytosis (MPC), and receptor-mediated endocytosis, including clathrin-mediated (CME) and caveolae-mediated (CvME). ${ }^{26}$ These endocytosis mechanisms are based on the molecule's size, properties, and internalization machinery. However for most cell types, nanoparticles are internalized via MPC, CME, and CvME. The pathway of MPC is a non-selective internalization of large volumes of extracellular medium where the cell membrane 
forms a pocket and pinches off a vesicle about 0.5-5 $\mu \mathrm{m}$ in diameter. ${ }^{31}$ The pathway of CME involves clathrincoated pits that form a polygonal clathrin lattice that bind ligands and molecules, where the clathrin-coated pits pinch off clathrin-coated vesicles about $100-150 \mathrm{~nm}$ in size. The clathrin-coated vesicles depolymerize going from early endosome to lysosome because of the drop in $\mathrm{pH} .{ }^{31}$ The pathway of CvME involves small hydrophobic domains with caveolin, which is a cholesterol binding protein. An approximately $50 \mathrm{~nm}$ caveolar vesicle forms a caveosome and follows a non-acidic and non-digestive intracellular route. ${ }^{31}$

Depending on the mechanism of uptake, the cell will either recycle out or sort and traffic the nanoparticles to a specific organelle for further processing. ${ }^{27}$ Recently, Mendez et al. demonstrated the subcellular localization and toxicity of CPNs were dependent on the chemical structure of the side chains and conjugated backbone. ${ }^{29}$ Cellular uptake and toxicity were modulated by adjusting amine-density of the side chain, primary vs. tertiary, and hydrophilicity, primary amine vs. triethylene oxide. Therefore, it is important to understand how the physicochemical and biophysical properties of nanocarriers affect the interaction with the cell membrane, and subsequent uptake and trafficking. Synthetic nanoparticle systems have the unique properties that allow for both passive and active targeting, making an excellent platform for improving therapeutics.

\subsection{Characterization of cellular interactions}

The first step to characterize biophysical properties is to ensure the material is non-toxic to target cells. The most commonly used assays measure viable cells, rather than dead cells, as there are several widely used and high throughput methods established. In general, cells are treated with a compound that when metabolized by viable cells forms a colored product that can be measured using a microplate reader, typically using absorbance.

When determining quantitative cellular uptake of nanoparticles, flow cytometry is used. Briefly, a fluid stream of cells is passed through a beam of light, and both fluorescence and scattered light are recorded. Fluorescence intensity relative to the cell gives the quantitative uptake of the nanoparticle. Scattered light is used to differentiate live cells from dead ones to obtain an accurate fluorescence value. Cellular uptake pathways can be determined by pre-treating cells with commercially available pharmacological inhibitors. ${ }^{32}$ Clathrin-mediated endocytosis can be distinguished under a potassium depletion condition or by treating with 
chlorpromazine, which dissociates clathrin coated pits on the cell surface and disrupts the regulation of actin cytoskeleton. Caveolae-mediated endocytosis can be distinguished when the presence of cholesterol is disrupted. Methyl- $\beta$-cyclodextrin is known to deplete cholesterol from the plasma membrane by forming inclusion complexes. Cholesterol is involved in both macropinocytosis and caveolae-mediated endocytosis. Genistein inhibits tyrosine kinases that are involved in the pinching of caveolae. LY2994 inhibits phosphatidylinositol 3-kinase, which recruits factors involved in macropinocytosis. Cytochalasin D inhibits actin polymerization, leading to actin filament disassembly, and this disassembly affects both macropinocytosis and caveolae-mediated endocytosis. ${ }^{32}$

Visualization of cellular uptake of fluorescent nanoparticles is achieved using high resolution microscopic imaging. Trafficking and subcellular localization can be evaluated by co-staining with standard fluorescent dyes as there are a wide variety of commercially available dyes to stain organelles. Co-localization of the dyes (i.e.; green CPN and red commercial dye) is defined as the co-occurrence indicated by the spatial overlap of the dyes. The correlation indicated by the co-distribution of the dyes in proportion to each other within and between structures. ${ }^{33}$ To evaluate quantitatively co-localization, the Pearson's Correlation Coefficient (PCC) method is used to determine the degree of association between the dye signals on a pixel-by-pixel basis. The PCC method takes into account the average intensity of each pixel and normalized so the PCC score is independent of dye brightness and background signal. PCC values range from +1 and -1 , indicating perfectly linearly and inversely correlated, respectively. ${ }^{34}$

\subsection{Summary}

By exploring nanoparticles for biological use, a better understanding of the biological hurdles and ways to improve nanoparticle design can be achieved. Nanoparticle design and improvement stems from a thorough characterization of material composition, size, shape, surface modification, and flexibility, which will ultimately determine biocompatibility. The aim of my dissertation is to better understand CPN structure and aggregation, and how those structural features affect cellular interactions to establish a structure-function relationship. Chapter 2 details the fabrication of core-shell nanoparticles using a semi-flexible cationic CPN complexed with a polyanion, hyaluronic acid (HA), which has a high affinity towards receptor overexpressed on cancer cells. The resulting fluorescent core-shell nanoparticles exhibited high cancer cell specificity with 
low adsorption to normal cells, compared to a rigid control CPN that formed random spherical complexes with HA. The dramatic difference in ionic complex formation based on the flexibility of CPN backbone led to the work in Chapter 3, which details a systematic investigation on the aggregation properties of CPNs that vary by side chain and backbone structures in response to different biologically important anionic polysaccharides. These differential interactions were analyzed using analytical statistical methods to demonstrate that structurally diverse CPNs can be used for the differentiation of biological molecules in a complex biological medium. Chapter 4 details the fabrication of a semi-flexible CPN modified with the mitochondrial-targeting triphenylphosphonium (TPP) group. The subcellular localization and cellular toxicity were affected by backbone flexibility and molecular weight, in a time-dependent fashion. In accordance with Chapter 2, the TPP-containing CPNs were treated with HA to modulate solubility and cellular interactions. Chapter 5 details the synthesis of dual-targeting red emissive CPNs grafted with folic acid (FA) side chains and complexed with hyaluronic acid (HA). Modulation of uptake kinetics and amount of internalized CPNs was achieved by the dual ligand-targeting CPNs designed for folate and hyaluronan receptors.

\subsection{References}

1. $\quad$ Yang, P.; Tarascon, J. M. Nat. Mater. 2012, 11, 560-563.

2. $\quad$ Langer, R.; Tirrell, D. A. Nature. 2004, 428, 487-492.

3. Alvarez, A.; Salinas-Castillo, A.; Costa-Fernandez, J. M.; Pereiro, R.; Sanz-Medel, A. Trends Anal. Chem. 2011, 30, 1513-1525.

4. $\quad$ Stobl, G. R. The Physics of Polymers; Springer-Verlag: Berlin, 2007.

5. $\quad$ Birks, J. B. Photophysics of Aromatic Molecules; Wiley-Interscience: London, 1970.

6. $\quad$ Zhu, C.; Liu, L.; Yang, Q.; Lv, F.; Wang, S. Chem. Rev. 2012, 112, 4687-4735.

7. Tuncel, D.; Demir, H. V. Nanoscale, 2010, 2, 484-494.

8. $\quad$ Evans, R. C. J. Mater. Chem. C. 2013, 1, 4190-4200.

9. Holding, S. Chromatography Today. 2011, 4, 4-8.

10. Chinchilla, R.; Najera, C. Chem. Soc. Rev. 2011, 40, 5084-121.

11. Yang, Y.; Zhao, L. Trends Anal. Chem. 2010, 29, 980-1003.

12. Cheng, Y. J.; Yang, S. H.; Hsu, C. S. Chem. Rev. 2009, 109, 5868-5923. 
13. Grimsdale, A. C.; Chan, K. L.; Martin, R. E.; Jokisz, P. G.; Holmes, A. B. Chem. Rev. 2009, 109, 897-1091.

14. McQuade, D. T.; Pullen, A. E.; Swager, T. M. Chem. Rev. 2000, 100, 2537-2574.

15. Dongen, S. F. M. v.; Hoog, H.-P. M. d.; Ruud J. R. W. Peters, M. N.; Nolte, R. J. M.; Hest, J. C. M. v. Chem. Rev. 2009, 109, 6212-6274.

16. Thomas III, S. W.; Joly, G. D.; Swager, T. M. Chem. Rev. 2007, 107, 1339-1386.

17. Filipe, V.; Hawe, A.; Jiskoot, W. Pharm. Res. 2010, 27, 796-810.

18. Shang, L.; Nienhaus, K.; Nienhaus, G. U. J. Nanobiotechnology. 2014, 12, 1-11.

19. Ediriwickrema, A.; Saltzman, W. M. ACS Biomater. Sci. Eng. 2015, 1, 64-78.

20. Ferrucci, J. T. Radiographics. 1998, 18, 1569-86.

21. Barenholz, Y. Curr. Opin Oncol. 2012, 24, 325-331.

22. Hawkins, M. J.; Soon-Shiong, P.; Desai, N. Adv. Drug Delivery Rev. 2008, 60, 876-885.

23. Ledet, G.; Mandal, T. K. U.S. Pharm. 2012, 37, 7-11.

24. O’Brien, M. E.; Socinski, M. A.; Popovich, A. Y.; Bondarenko, I. N.; Tomova, A.; Bilynsky, B. T.; Hotko, Y. S.; Ganul, V. L.; Kostinsky, I. Y.; Eisenfeld, A. J.; Sandalic, L.; Oldham, F. B.; Bandstra, B.; Sandler, A. B.; Singer, J. W. J. Thorac. Oncol. 2008, 3, 728-734.

25. Dinndorf, P. A.; Gootenberg, J.; Cohen, M. H.; Keegan, P.; Pazdur, R. Oncologist 2007, 12, 991-998.

26. Zhao, F.; Zhao, Y.; Liu, Y.; Change, X.; Chen, C.; Zhao, Y. Small. 2011, 7, 1322-1337.

27. Watson, P.; Jones, A. T.; Stephens, D. J. Adv. Drug Deliv. Rev. 2005, 57, 43- 61.

28. Ko, J. Y.; Mendez, E.; Moon, J. H. Macromolecules. 2011, 44, 5527-5530.

29. Mendez, E.; Moon, J. H. Chem. Commun. 2013, 49, 6048-6050.

30. Shi, J.; Votruba, A. R.; Farokhzad, O. C.; Langer, R. Nano Lett. 2010, 10, 3223-3230.

31. Sing, J.; Michel, D.; Chitanda, J. M.; Verral, R. E.; Badea, I. J. Nanobiotechnology 2012, 10 , 1-14.

32. Kuhn, D. A.; Vanhecke, D.; Michen, B.; Blank, F.; Gehr, P.; Pteri-Fink, A.; RothenRutishauser, B. J. Nanotechnol. 2014, 5, 1625-1636.

33. Dunn, K. W., Kamocka, M. M.; McDonald, J. H. Am J Physiol Cell Physiol. 2011, 300, C723C742.

34. Bolte, S.; Cordelieres, F. P. Journal of Microscopy. 2006, 224, 213-232.

35. Waters, M. L., Curr. Opin. Chem. Biol. 2002, 6, 736-741.

36. Stockhofe, K.; Postema, J. M.; Schieferstein, H.; Ross, T. L. Pharmaceuticals. 2014, 7, 392-418. 


\section{CHAPTER II}

Fabrication of core-shell nanoparticles via controlled aggregation of semi-flexible conjugated polymer and hyaluronic acid

Reproduced in part with permission from Macromolecules 2013, 46, 6374-6378.

Copyright (C) 2013

American Chemical Society

Megan Twomey ${ }^{\dagger}$, Yoonmi Na ${ }^{\dagger}$, Zahilyn Roche ${ }^{\dagger}$, Eladio Mendez ${ }^{\dagger}, N_{a m u n a ~ P a n d a y}^{\ddagger}$, Jin He ${ }^{\ddagger}$, and Joong Ho Moon*广

${ }^{\dagger}$ Department of Chemistry and Biochemistry, Florida International University, Miami, Florida 33199, United States

${ }^{\ddagger}$ Department of Physics, Florida International University, Miami, Florida 33199, United States

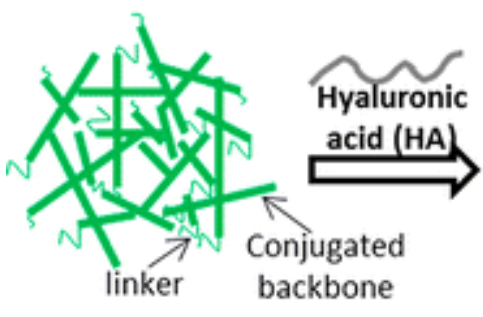

Semi-flexible PPB

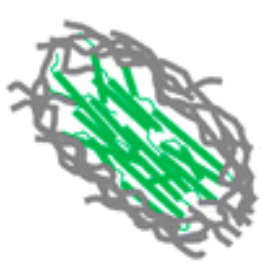

Core-shell nanoparticles

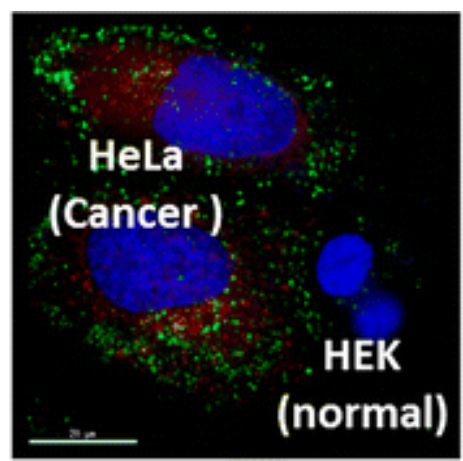




\subsection{Abstract}

Core-shell conjugated polymer nanoparticles (CPNs) were fabricated by complexing a semi-flexible conjugated polymer (CP) with hyaluronic acid (HA). Incorporation of a small amount of flexible unit into the rigid rod conjugated backbone introduces conjugated backbone reorganization under ionic complexation to increase $\pi-\pi$ interaction, resulting in core-shell nanoparticles with a hydrophobic core wrapped with a HA shell. The core-shell nanoparticles exhibited no cellular toxicity and high cancer cell specificity with minimal binding to normal cells.

\section{$2.2 \quad$ Introduction}

Recently, conjugated polymers (CPs) have attracted much attention for sensing, ${ }^{1-4}$ labeling, ${ }^{5-10}$ and delivery ${ }^{11-13}$ of biological substances owing to their excellent photophysical and biophysical properties. CPs are synthetic macromolecules containing fully conjugated $\pi$-electrons along the backbones, resulting in high luminescence brightness, high photostability, and excellent energy transfer. ${ }^{14,15}$ By incorporating hydrophilic polar side chains and functional entities such as sensing units or targeting ligands, various conjugated polyelectrolytes (CPEs) and conjugated polymer nanoparticles (CPNs) have been synthesized and used for biological applications in aqueous environments. ${ }^{16-18}$

The photophysical excellence and robustness of CP-based nanomaterials are typically useful for labeling target cells or tissues. Biophysically, amphiphilicity of the CPEs and CPNs is important for cellular interaction and subsequent cellular entry because the cell surface contains both negatively charged proteoglycans and hydrophobic membrane lipids. Especially, particles with high surface-to-volume ratios exhibit size, shape, and functional group-dependent cellular interactions and entry efficiency. ${ }^{19,20}$ Therefore, functional modifications and structural modulations of CP-based nanomaterials are highly anticipated to obtain desired biophysical properties for improved cellular applications.

Previously, we fabricated positively charged CPNs by treating a nonaqueous soluble, primary aminecontaining rigid rod CP with organic acids followed by dialysis. ${ }^{21,22}$ The aggregation structures of CPNs, after complete removal of organic acids, were dependent on the nature of organic acids. Acetic acid treatment 
produced loose aggregation of rigid rod $\mathrm{CP}$ backbones, while increased $\pi-\pi$ interactions among the backbones were observed when the same batch of polymer was treated with tartaric acid (i.e., dicarboxylic acid). From this observation, we hypothesized that if CPs containing flexible units along the backbones are treated with polymeric acids, $\pi-\pi$ interaction among the backbones will be dramatically increased because the semiflexibility of the nonaqueous soluble backbones will help backbone reorganization to maximize hydrophobic chain interaction. If complexation between the nonaqueous soluble $\mathrm{CP}$ and polyanion contributes to increasing aqueous solubility of CPs, random complex formations is expected with no significant spectral changes. To test this hypothesis and functionalize the CP with cancer cell-specific ligands, we employed a linear polysaccharide, hyaluronic acid (HA), which contains N-acetyl-D-glucosamine and Dglucuronic acid units. HA has specific binding with cell surface receptors such as CD44 and RHAMM, which are overexpressed in many cancer cells. ${ }^{23}$ Numerous cancer drugs and polymers have been modified with HA for targeted drug and gene delivery. ${ }^{24-28}$

In this chapter, core-CP-shell-HA nanoparticles are obtained when semi-flexible $\mathrm{CPs}$ are treated with polyanionic HA, while nonflexible CPs produce random complexes upon HA treatment. The core-shell nanoparticles are nontoxic to cells and exhibit high cancer cell specificity through the specific binding of HA to cancer cell surface receptors.

\subsection{Results and Discussion}

A semi-flexible poly(phenylenebutadiynylene) (PPB), which contains a small fraction of flexible nonconjugating units along the rigid conjugated backbones, was prepared by following our published method $^{21}$ and used for the complexation with HA (Figure 2.1). The semi-flexible PPB was treated with organic acids, dialyzed against water, and complexed with HA. The semi-flexible PPB exhibits characteristic photophysical properties of the fully conjugated PPBs because the amount of nonconjugated unit is limited ( $\sim 5-10 \%)$ not to perturb the $\pi$ - electron conjugation along the backbone. 

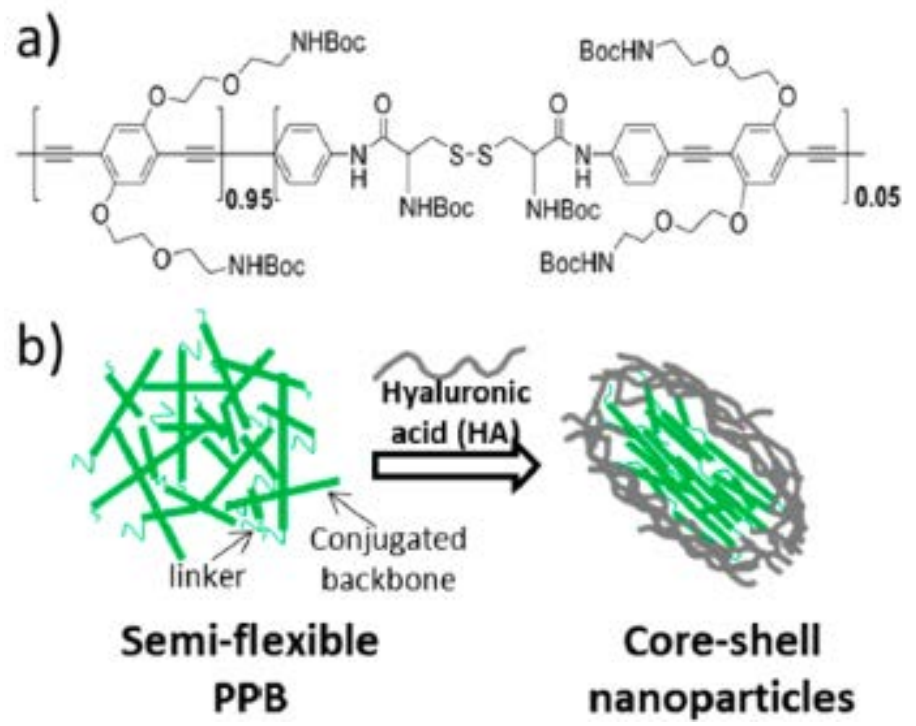

Figure 2.1. (a) Chemical structure of the semi-flexible PPB. (b) A schematic presentation of structural reorganization of the semi-flexible PPB upon HA complexation. The semi-flexible PPB in water was prepared by deprotection of Boc group using organic acid treatments followed by dialysis.

The photophysical and physical properties of the semi-flexible PPB in water were similar to the characteristics of loosely aggregated CPs with fully conjugated backbones. When the semi-flexible PPB was mixed with HA, the resulting complex exhibited dramatic changes in both absorption and emission spectra. As shown in Figure 2.2, a new strong absorption peak appeared at $\sim 474 \mathrm{~nm}$ (30 nm red-shifted upon complexation) (Figure 2.2a), and the emission intensity of the complex decreased significantly (Figure 2.2b). We believe that unlike complexes formed between conjugated rigid oligomers and polysaccharides, which exhibit both red-shift and fluorescent increase through chain end-to-end alignment induced by linear polysaccharides, ${ }^{29,30}$ the loosely aggregated, nonaqueous soluble PPBs connected with flexible linkers were able to reorganize to increase interpolymer $\pi-\pi$ interactions, similarly to the high molecular weight, fully conjugated poly(phenyleneethynylene) (PPEs) at the air-water interface under mechanical forces. ${ }^{31}$ As a control, a fully conjugated PPE containing the same amine side chains was treated with HA, and the spectroscopic behaviors were monitored. We employed the structurally similar PPE as a control due to poor solubility of PPB without flexible linkers. Upon complexation with HA, the control PPE exhibited only a small increase in the absorption maximum $(<5 \mathrm{~nm})$ without a spectral shape change (Figure 2.2c), and the fluorescent intensity decreased slightly (Figure 2.2d). This implies that no significant structural 
reorganization of PPE occurred upon HA complexation, supporting that the semiflexibility of PPB is responsible for the significant spectral changes upon ionic complexation.

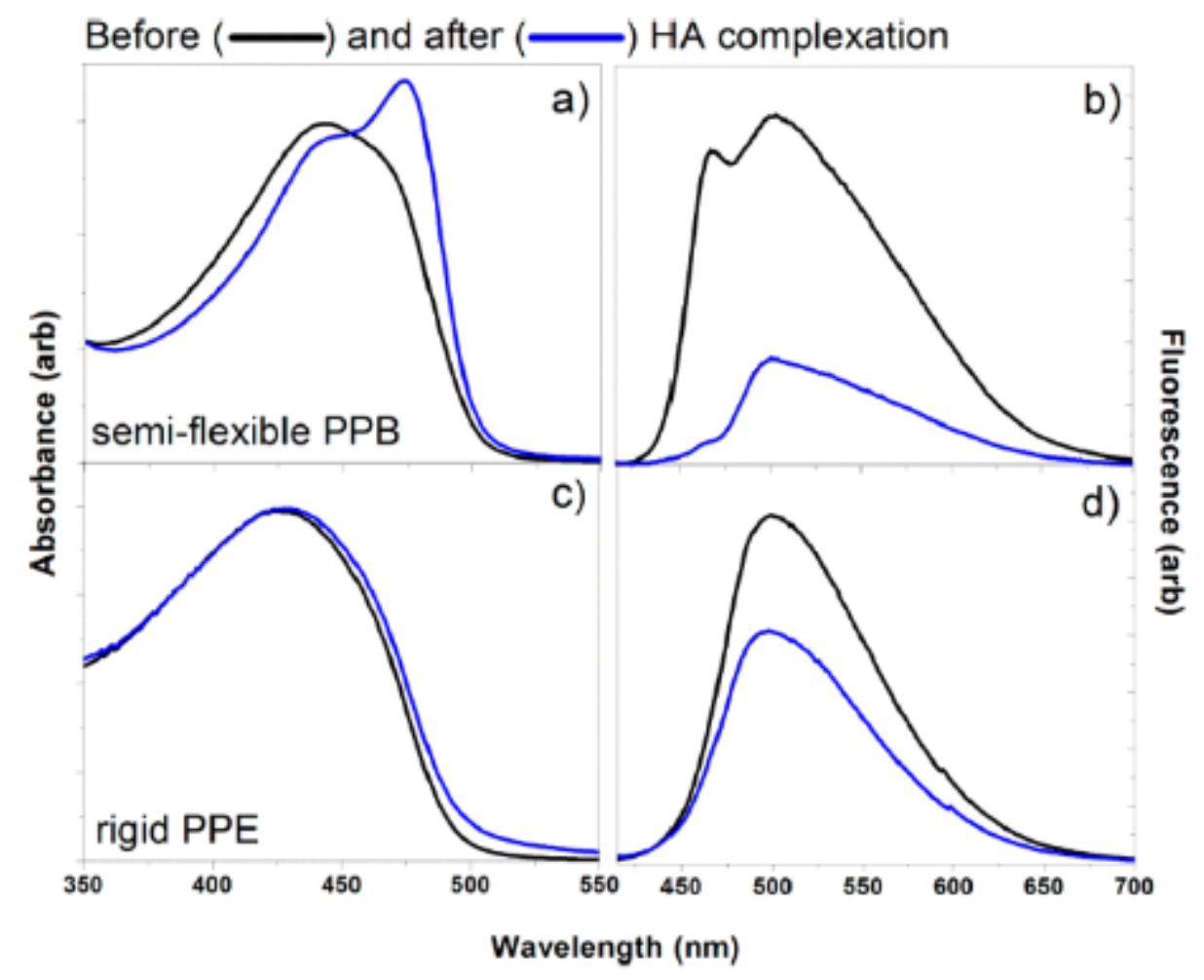

Figure 2.2 Absorbance and emission spectra of semi-flexible PPB (a, b) and rigid PPE (c, d) before and after HA complexation. A sharp absorption peak at $474 \mathrm{~nm}$ (a) and decreased emission intensity of the semiflexible PPB after HA complexation supports the formation of ordered aggregation among the semi-flexible conjugated backbones. No significant changes were observed upon HA complexation of the control PPE (c, d).

While the hydrodynamic radius of the semi-flexible PPB at the low concentration $(10 \mu \mathrm{M})$ could not be determined due to insufficient scattering intensity from the loose aggregations, the ionic complexes produced strong light scattering, implying the formation of dense particles. The hydrodynamic radius of the semiflexible PPB/HA complex was successfully determined to be $51.35 \pm 0.77 \mathrm{~nm}$. Zeta potential value of the semi-flexible PPB/HA nanoparticles in water at neutral $\mathrm{pH}$ was $-25.5 \pm 11.7 \mathrm{mV}$ (Supporting Information Figure S2.1). The hydrodynamic radius of the control PPE/HA complex was determined to be $79.49 \pm 0.18$ $\mathrm{nm}$, with a zeta potential of $-32.6 \pm 5.6 \mathrm{mV}$ (Figure S2.2). The control PPE/HA was almost $30 \mathrm{~nm}$ larger than the semi-flexible PPB/HA, implying that more loosely aggregated particles were formed. 
Atomic force microscopic (AFM) imaging of the semi-flexible PPB/HA nanoparticle supported the formation of elongated core--shell nanoparticles with average size of $58 \pm 13 \mathrm{~nm}$ (by measuring lateral sizes), as shown in Figure 2.3c, while the semi-flexible PPB without HA complexation exhibited mixed particles with no specific shapes (Figure 2.3a). AFM phase image also showed that core-shell particles were favorably formed at a molar ratio of 1:3 of the semi-flexible PPB to HA. Both at the lower and higher ratios, no defined core-shell nanoparticles were observed (Figure 2.4a). Higher density (represented as dark color) was observed in the center of the nanoparticles, while lower density (bright color) was observed in the shell. Since the particles were prepared on an aminosilanized mica surface, it is difficult to determine the shape and size of the intact nanoparticles in water by the AFM imaging. The AFM images are believed to be flattened during the sample preparation determined by the height analysis (Figure 2.4b). The topographic and phase images of the control PPE/HA show circular particles with no core-shell shape, with an average size and height of $81 \pm 9$ and $4 \pm 0.5 \mathrm{~nm}$, respectively (Figure S2.3). The AFM imaging of the control PPE/HA further supports that HA does not cause structural reorganization of rigid rod CPs.
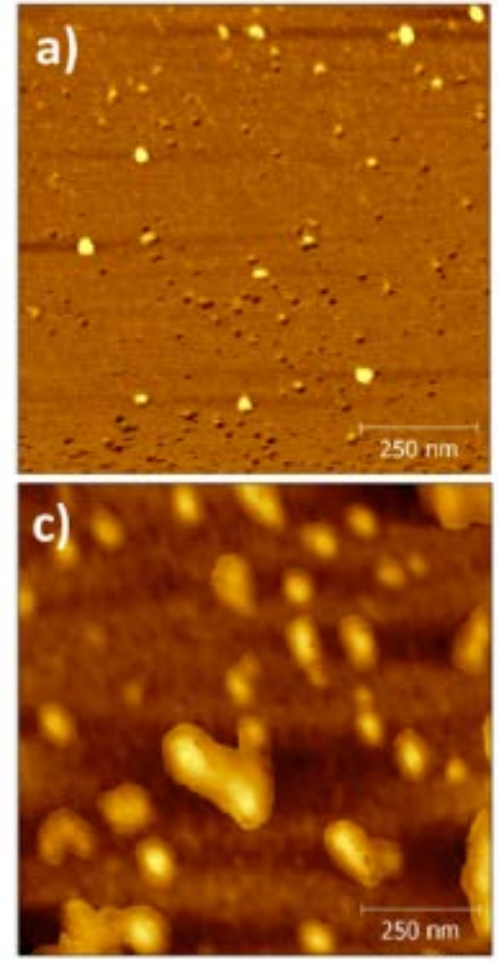
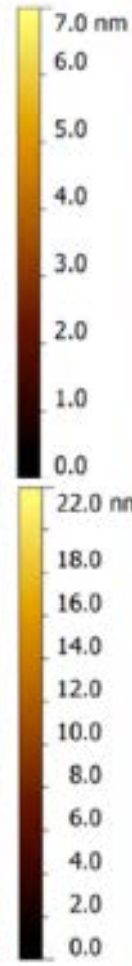
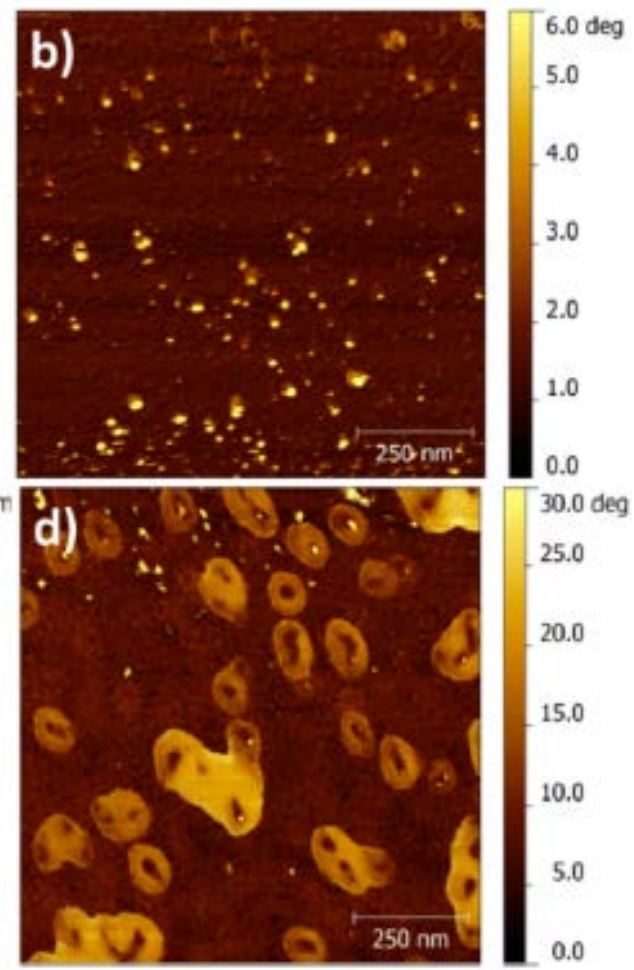

Figure 2.3. AFM topographic (a, c) and phase (b, d) images of the semi-flexible PPB (a, b) and semi-flexible PPB/HA nanoparticles formed at 1:3 molar ratio (c, d). The semi-flexible PPB/HA exhibits elongated particles on a mica surface (c), and the phase image (d) reveals that the complexes are core-shell nanoparticles. 

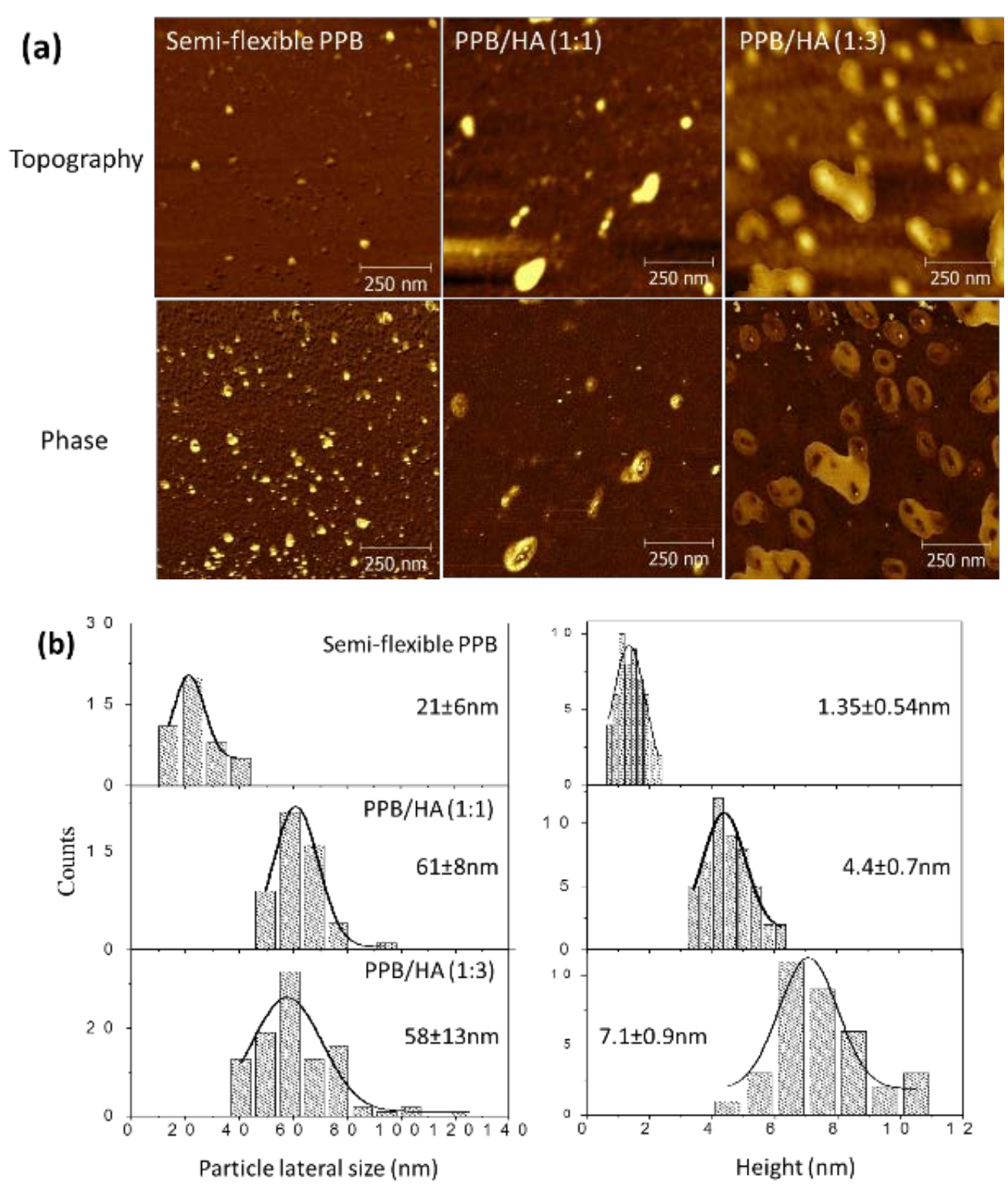

Figure 2.4. Topography and phase images (a) and size analysis histograms (b) of semi-flexible PPB, semiflexible PPB/HA (1:1), and semi-flexible PPB/HA (1:3), respectively.

Cancer cell specificity of the core-shell nanoparticle was examined by incubating various cells including cancer and normal cells with the core-shell nanoparticles for different times, and the normalized mean fluorescent intensity ratios to the control cells were plotted by analyzing flow cytometry data (Figure 2.5). As shown in Figure 2.5, cancer cells overexpressing CD44 [i.e., human cervical carcinoma (HeLa) and human pancreatic carcinoma cell lines (Panc-1)] exhibited an intensity increase as the incubation time increased, while normal human embryonic kidney cells (HEK) and cancer cells with low CD44 expression 
exhibited low fluorescent intensity over the incubation times. The labeling specificity coincided well with the CD44 expression levels, which were measured by incubating fluorescently labeled antibody against CD44 with various cells (inset of Figure 4). As expected, the core-shell nanoparticles exhibit no toxicity measured by ATP consumption of the treated cells (Figure S2.4).

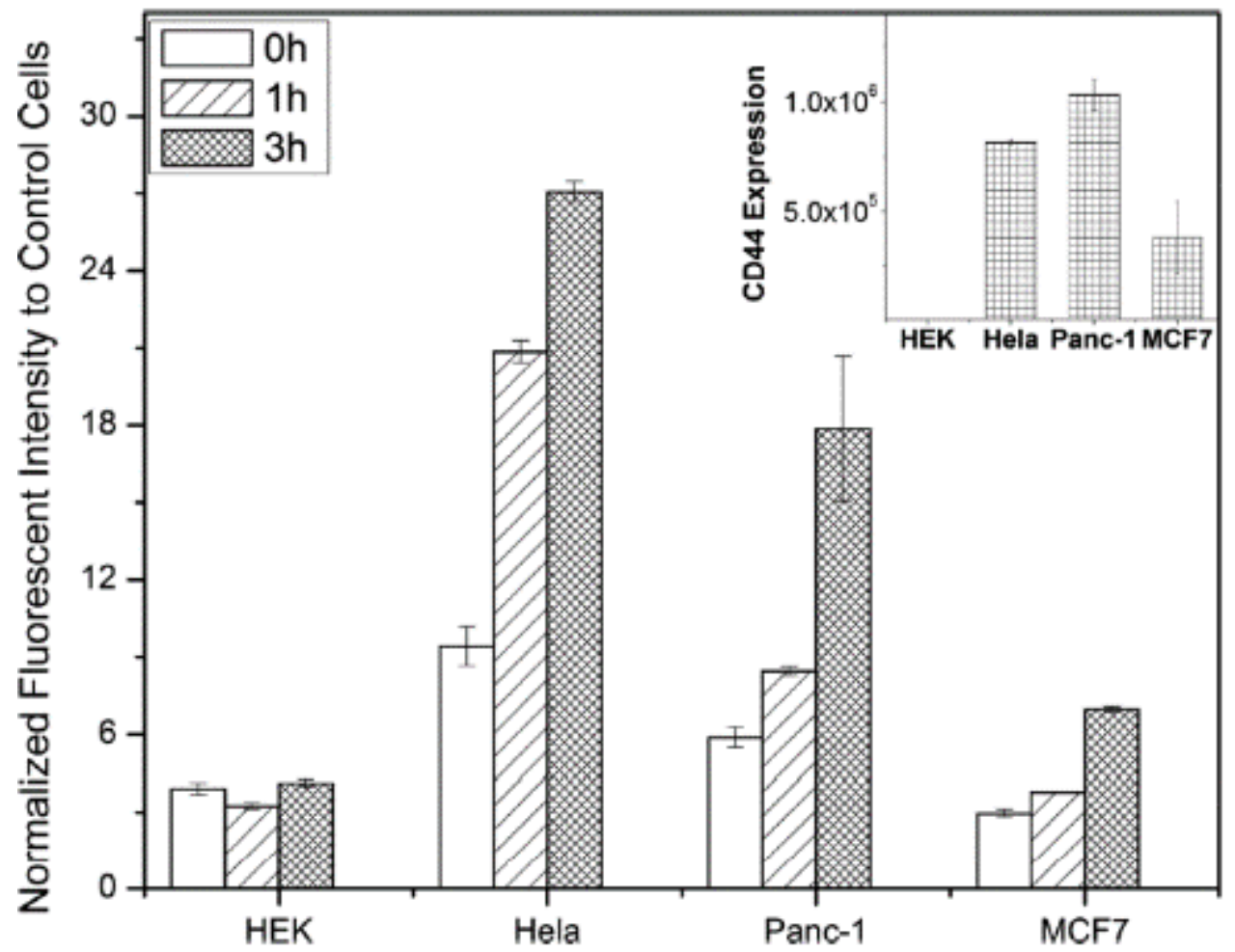

Figure 2.5. Normalized fluorescent mean intensity ratios of various cells treated with the semi-flexible PPB/HA (1:3) up to $3 \mathrm{~h}$. Cancer cells overexpressed CD44 (inset) exhibit higher fluorescent intensities than those from cells with low CD44 expression, supporting specific labeling of cancer cells via HA-CD44 interaction.

The specific labeling of cancer cells by the core-shell particles was further confirmed by fluorescence microscopic imaging of cancer cells co-cultured with normal HEK cells. Before co-culturing, HeLa cells were pre-labeled with a fluorescent dye (CellTracker Red) to fluorescently distinguish the cancer cells from normal cells. It is known that some fluorescent dyes have poor retention in the cell and can readily diffuse through the cell membrane. CellTracker Red was used because it is converted into an impermeable dye by forming covalent bonds inside cells. After incubating the cells for $1 \mathrm{~h}$, the cells were rinsed, stained (for labeling of nucleus), and fixed for microscopic imaging. Because the cancer cells possibly influence the normal cell growth, each cell line was independently cultured and incubated with the core-shell nanoparticles 
under the same incubation condition. As shown in Figure 2.6, strong green fluorescent signals were observed as scattered dots throughout the HeLa cells (pre-labeled with a red fluorescent dye), while much weaker green signals were observed from the normal HEK cells, indicating that the core-shell nanoparticles preferentially labeled HeLa cells through the specific HA-CD44 binding followed by endocytosis. For the control cells cultured independently, HeLa cells exhibited many green fluorescent dots throughout the cytosol (Figure 2.6, row 3), while the number and intensity of green dots observed from HEK were significantly low (Figure 2.6, row 2).

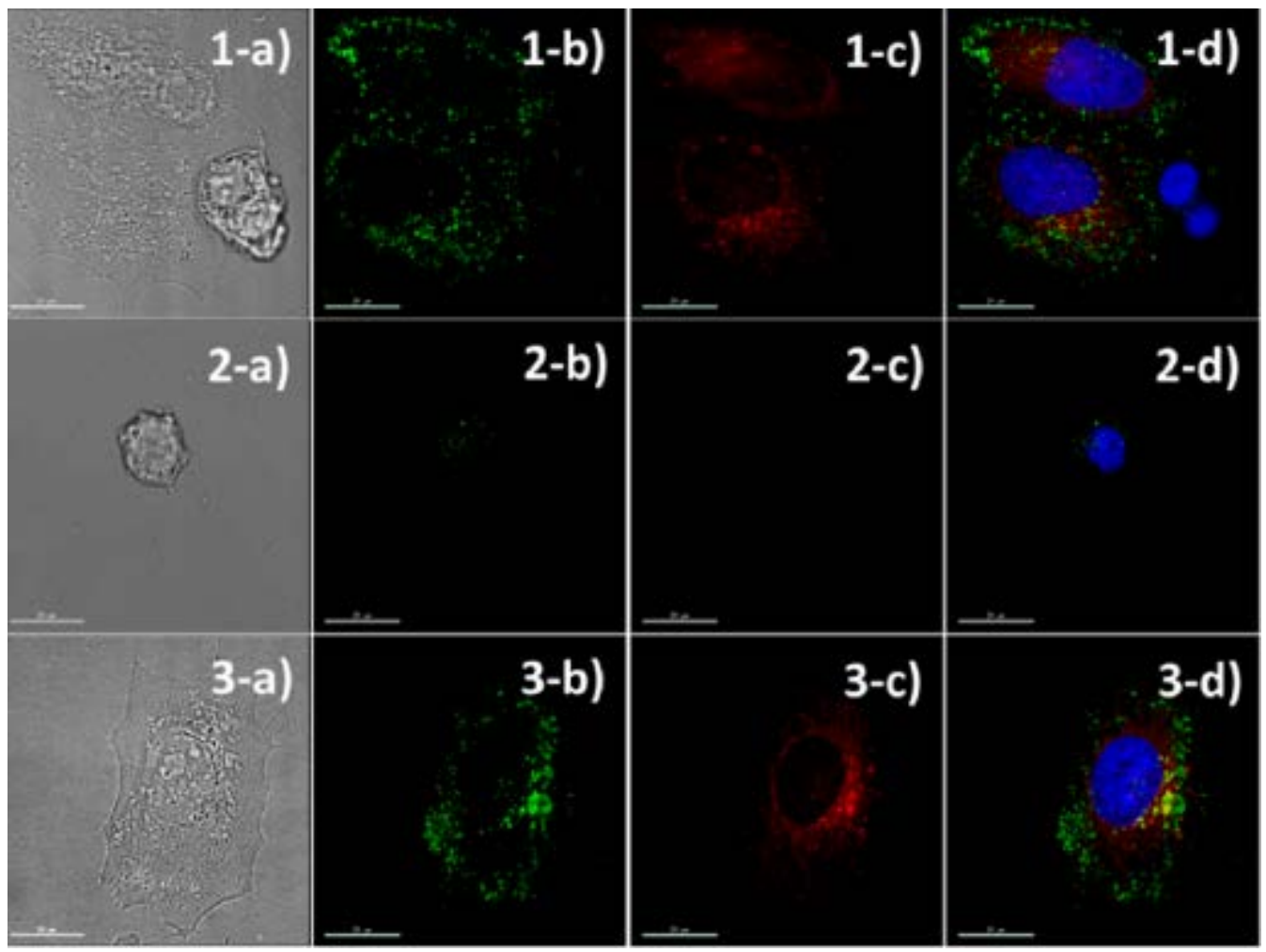

Figure 2.6. Fluorescent microscopic $(b-d)$ and contrast (a) images of HeLa/HEK mixed cells (1), HEK cells (2), and HeLa cells (3) incubated with the semi-flexible PPB/HA for $1 \mathrm{~h}$. HeLa cells were fluorescently prelabeled with a red dye (column c) before the core-shell nanoparticle incubation. Core-shell nanoparticles were seen under the green channel (column b); the nuclei were stained with a blue dye; and merged images were seen in column $d$. The core-shell nanoparticles preferentially labeled HeLa cells while exhibiting low binding to normal HEK cell.

\subsection{Conclusion}

In summary, core-shell nanoparticles were fabricated by complexing a semi-flexible PPB with a linear polysaccharide, HA. The resulting fluorescent core-shell nanoparticles exhibited high cancer cell specificity with low adsorption to normal cells. Since the size and shape of nanomaterials significantly influence labeling 
and delivery efficiency of biological substances, the concept of core-shell nanoparticle formation through controlled aggregation introduced in this work will contribute to novel biomaterials syntheses and fabrications.

\subsection{Outlook}

This chapter presents a strategy for modulating particle shape using ionic complexation in a backbone flexibility-dependent fashion. Since the publication of this work in Macromolecules, a systematic investigation on ionic complexation of CPNs that vary by side chain and backbone structure was performed (detailed in Chapter 3). The subcellular localization of amine-containing CPNs, including PPE and PPBbased, demonstrated side chain and backbone-dependent cellular uptake. ${ }^{32}$ The semi-flexible PPB polymer showed increased Golgi uptake, compared to non-flexible amine-containing CPNs. The semi-flexible CPN also showed improved uptake and accumulation, demonstrating that controlled subcellular localization leads to enhanced internalization. The cellular uptake subcellular localization of the semi-flexible CPN complexed with HA and a similar polyanion, carboxymethyl cellulose (CMC), was explored. While in this chapter, the semi-flexible CPN displayed enhanced targeting of cancer cells over normal cells, there was no significant increase in internalization of the polyplexes with HA and CMC. Complexation of the semi-flexible CPN with HA and CMC led to increased accumulation in late endosome/lysosome as expected based on the dramatic changes from spherical to core-sell particles. While HA complexation leads to increased labelling of cancer cells, a strategy to improve targeting and increase internalization of CPNs is needed. A dual ligand approach for targeting of folate and hyaluronan receptors is detailed in Chapter 5.

\subsection{Experimental}

Atomic force microscopy (AFM) experiments and analysis were performed by Namuna Panday. Toxicity assays and flow cytometry experiments were performed by Yoonmi Na. Microscope imaging and processing was completed by Dr. Eladio Mendez.

2.6.1 General: Chemicals and solvents were purchased from Fisher Scientific and used as received. Deuterated solvents were purchased from Cambridge Isotope Laboratories (Cambridge, MA). Molecular weight was determined using gel permeation chromatography (GPC) against polystyrene standards on a Shimadzu HPLC system equipped with PLgel $5 \mu \mathrm{m}$ MIXED-D columns and SPD-20A ultraviolet-visible 
(UV-vis) detector. The purification of the CPN was conducted using an Ultrafiltration Stirred Cell (Millipore) with a $30 \mathrm{kDa}$ molecular weight cut-off (MWCO) membrane (Ultracel ultrafiltration disc). UV-Vis spectrum was recorded using Varian Cary 50 Bio spectrophotometer. Fluorescence spectrum was obtained using a FluoroLog-3 Spectrofluorometer (Jobin Yvon/Horiba). NMR spectrum was recorded on a $600 \mathrm{MHz}$ Avance Bruker NMR spectrometer using a 5 mm BBI probe at 298 K. Fourier transform infrared (FT-IR) spectrum was obtained on a PerkinElmer Spectrum 100 FT-IR Spectrometer, using sample mounted on an attenuated total reflection cell.

2.6.2 Synthesis of control PPE without flexible linker: A Schlenk flask was charged with M1 (41mg, 0.056, 1.0 equiv) and M2 (30 mg, $0.056 \mathrm{mmol}, 1.0$ eqiv) and, along with $\mathrm{Pd}\left[\left(\mathrm{PPh}_{3}\right)_{2} \mathrm{Cl}_{2}\right]$ (4 mg, $0.0056 \mathrm{mmol}$ ,0.1 equiv) and CuI (0.3 mg, $0.0028 \mathrm{mmol}, 0.05$ equiv), as shown in Scheme S2.1.

Scheme S2.1. Synthesis of control PPE without flexible linker.

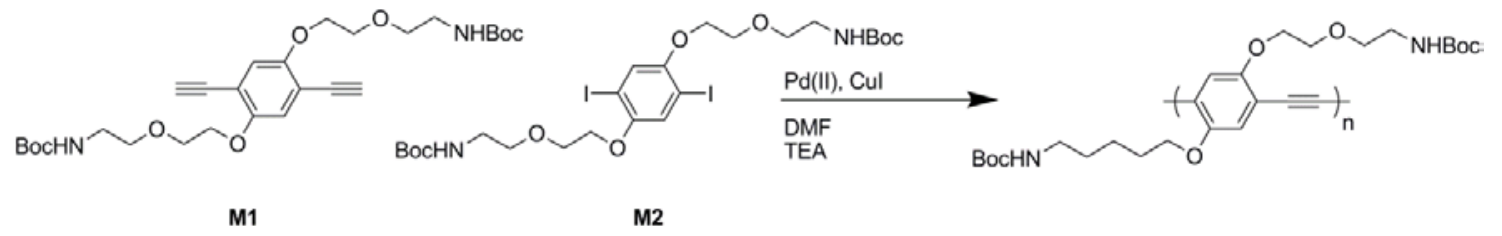

The Schlenk flask was evacuated and filled with $\mathrm{N}_{2}$ three times. A solution of anhydrous dimethylformamide (DMF) (4 mL) and freshly distilled triethylamine $(1 \mathrm{~mL})$ was degassed, and $1 \mathrm{~mL}$ of the mixed solution was transferred to the Schlenk flask using a cannular needle. The reaction was heated at $70{ }^{\circ} \mathrm{C}$ for $14 \mathrm{~h}$. The solution was then cooled to room temperature and transferred dropwise to cold ether, resulting in precipitation. After centrifugation (5 min, $4000 \mathrm{rpm}$ ), the supernatant was decanted, the precipitate was collected and dried to yield product. Yield: $44 \mathrm{mg}, 73 \% .{ }^{1} \mathrm{H}$ NMR (400 MHz, DMSO-d 6 , $\delta$ ): 7.16 (s, $1 \mathrm{H}$, ArH), 6.72 (s, 0.2H, NH-Boc), 4.21 (br, 1.84H, Ar- $\mathrm{OCH}_{2}$ ), 3.80 (br, 2.16H, $\mathrm{CH}_{2} \mathrm{O}$ ), 3.66 (br, 1.34H, $\mathrm{OCH}_{2}$ ), 3.52 (br, 2.22H, $\left.\mathrm{CH}_{2} \mathrm{NH}\right), 1.37$ (s, 7.33H, C( $\left.\left(\mathrm{CH}_{3}\right)_{3}\right)$; FT-IR (Neat): v = 3366 (br), 2933 (w), 2865 (s), 1704 (vs; C=O), 1507 (vs), 1457 (w), 1423 (w), 1365 (s), 1277 (w), 1247 (w), 1219 (s), 1172 (w), 1108 (s), 1057 (s), 1026 (w), 945 (s), $860 \mathrm{~cm}^{-1}$ (m); UV-vis (DMF): $\lambda_{\max } 432 \mathrm{~nm}$; fluo $\lambda_{\max }(400 \mathrm{~nm}$ ex) $=475 \mathrm{~nm}$; QY = 37\%; GPC Mn: 11.6 kDa, Mw: 16.4 kDa , PDI: 1.41.

Deprotection of Boc groups was carried out by mixing the polymer solution with acetic acid $(2 \mathrm{~mL})$ and trifluoroacetic acid $(1 \mathrm{~mL})$ and allowed to stir at room temperature for 14 days. The mixture was then added 
to acetic acid (20 mL), allowed to stir overnight, and centrifuged, and supernatant was added drop wise (2 drops/s) to $500 \mathrm{~mL}$ water $(18 \Omega)$ while stirring. Using a solvent-resistant stir cell fitted with a $30 \mathrm{kDa}-\mathrm{MWCO}$ membrane, the solution was concentrated to approximately $10 \mathrm{~mL}$, and dialyzed against $1 \mathrm{~L}$ of water. The resulting solution was further dialyzed in a $10 \mathrm{KDa}$ membrane for 3 days. ${ }^{1} \mathrm{H}$ NMR $\left(600 \mathrm{MHz}, \mathrm{D}_{2} \mathrm{O}, \delta\right): 7.03$ (br, 1H, Ar-H), 4.24-2.76 (br, 10.74H, $\mathrm{CH}_{2} \mathrm{CH}_{2} \mathrm{OCH}_{2} \mathrm{CH}_{2}$ ), 1.36 (s, 0.46H); FT-IR (Neat): $v=3417$ (br), 2925 (w), 2865 (s), 1647 (br), 1495 (w), 1462 (w), 1418 (w), 1357 (w), 1283 (w), 1205 (s), 1094 (s), 1040 (w), $936(\mathrm{w}), 841(\mathrm{w}) \mathrm{cm}^{-1}$; UV-vis $\left(\mathrm{H}_{2} \mathrm{O}\right): \lambda_{\max } 427 \mathrm{~nm}$; fluo $\lambda_{\max }(400 \mathrm{~nm}$ ex $)=492 \mathrm{~nm}$; QY $=2 \%$.

2.6.3 CP/HA complex formation: Sodium hyaluronate (MW $100 \mathrm{~K}$ ) was purchased from Lifecore and used as received. A stock solution was prepared by dissolving $2 \mathrm{mg}$ of $\mathrm{HA}$ in $1 \mathrm{~mL}$ of deionized water. The $\mathrm{CP} / \mathrm{HA}$ complex was formed by mixing $\mathrm{CP}(10 \mu \mathrm{M})$ with various molar equivalents of HA (i.e., 1:1, 1:3, and 1:9) for $1 \mathrm{~h}$.

2.6.4 Dynamic Light Scattering: Dynamic light scattering and zeta potential experiments were performed by Zetasizer nano-ZS (Zen 3600, Malvern Instruments Ltd.) using a microcuvette and a folded capillary cell (Catalog \# DTS1060), respectively, at room temperature. At least three independent samples were prepared and each sample was measured three times.

2.6.5 Atomic Force Microscopy: (3-aminopropyl)triethoxysilane (APTES) was vapor deposited to a freshly cleaved mica surface for $80 \mathrm{~min}$ in a desiccator filled with dry Argon. $30 \mu \mathrm{L}$ of CP/HA complex in DI water was placed on the APTES coated mica and incubated for 30-45 min in a laminar flow hood. The droplet was then rinsed away with $\sim 1 \mathrm{~mL}$ DI water (18 $\mathrm{M}$ ohm) and dried gently with dry Argon. Images were acquired with a MultiMode5 AFM microscope (Bruker, Santa Barbara, CA) operated in tapping mode in air using a 1.58-1.62 V oscillation amplitude with uncoated silicon AFM tips (T190, vistaprobes, $\mathrm{k} \sim 40 \mathrm{~N} / \mathrm{m}$ ) at a resonance frequency of $190 \mathrm{kHz}$. Typically, areas of $1 \times 1 \mu \mathrm{m}^{2}$ were scanned at a rate of $0.5-1 \mathrm{~Hz}$ and a resolution of $512 \times 512$ pixels. All the experiments were performed at room temperature. The images were further processed by Image Analysis Software Gwyddion. 


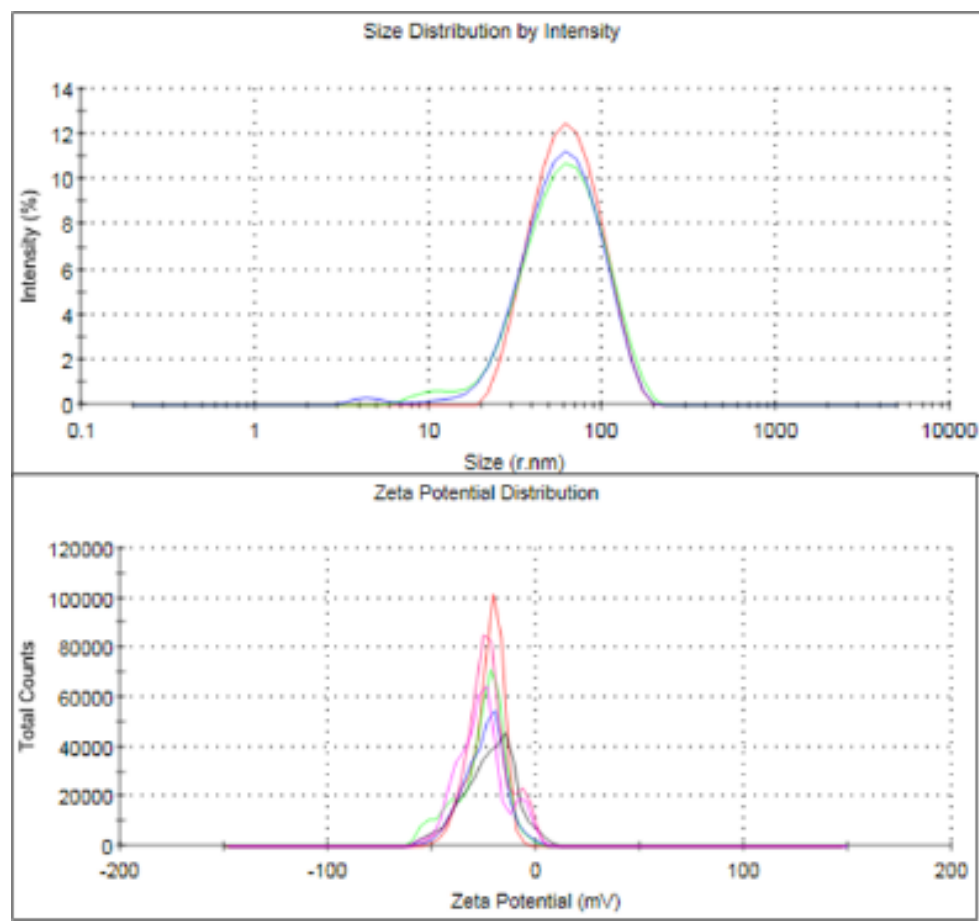

Figure S2.1. DLS (top) and zeta potential (bottom) of the semi-flexible PPB/HA complex. Hydrodynamic radius was measured with three replicates and zeta potential was measured with six replicates.

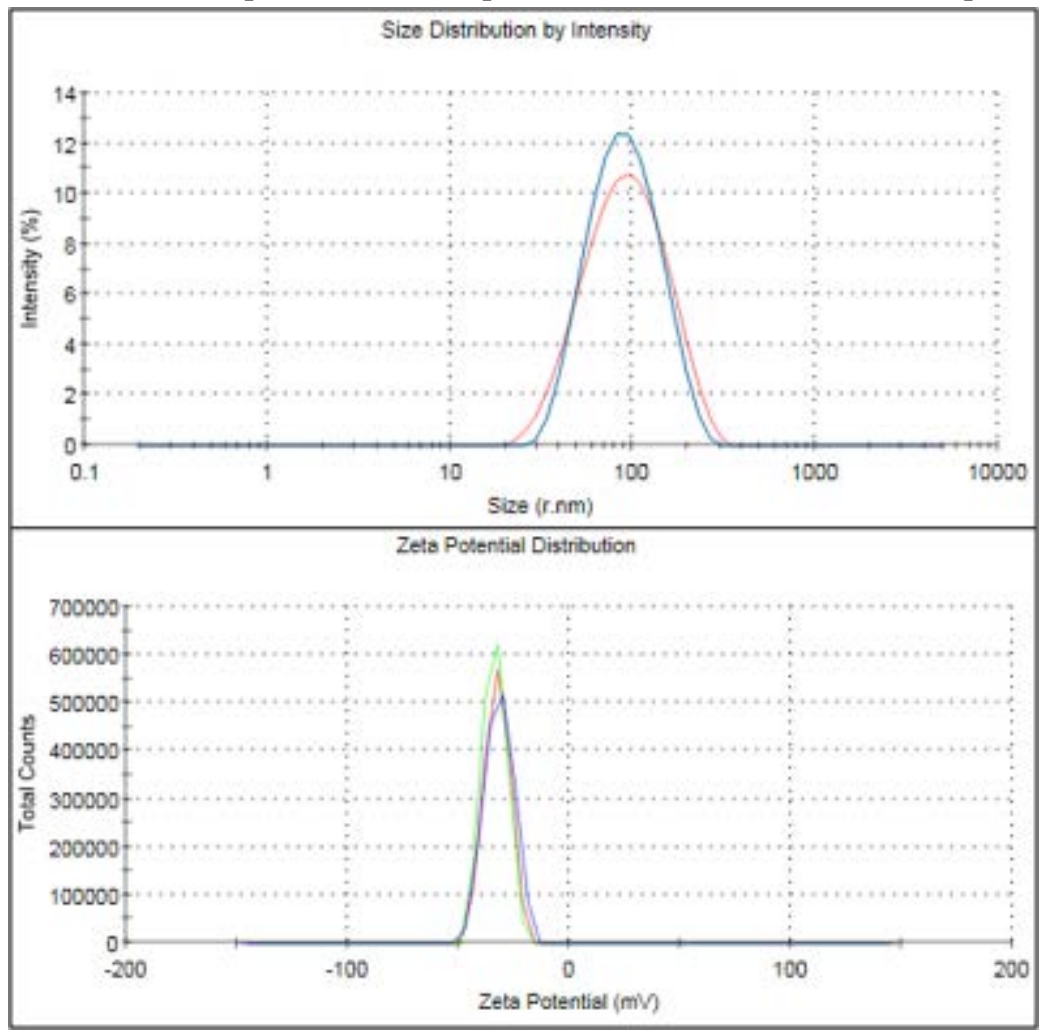

Figure S2.2. DLS (top) and zeta potential (bottom) of the control PPE/HA complex. Hydrodynamic radius was measured with three replicates and zeta potential was measured with six replicates. 

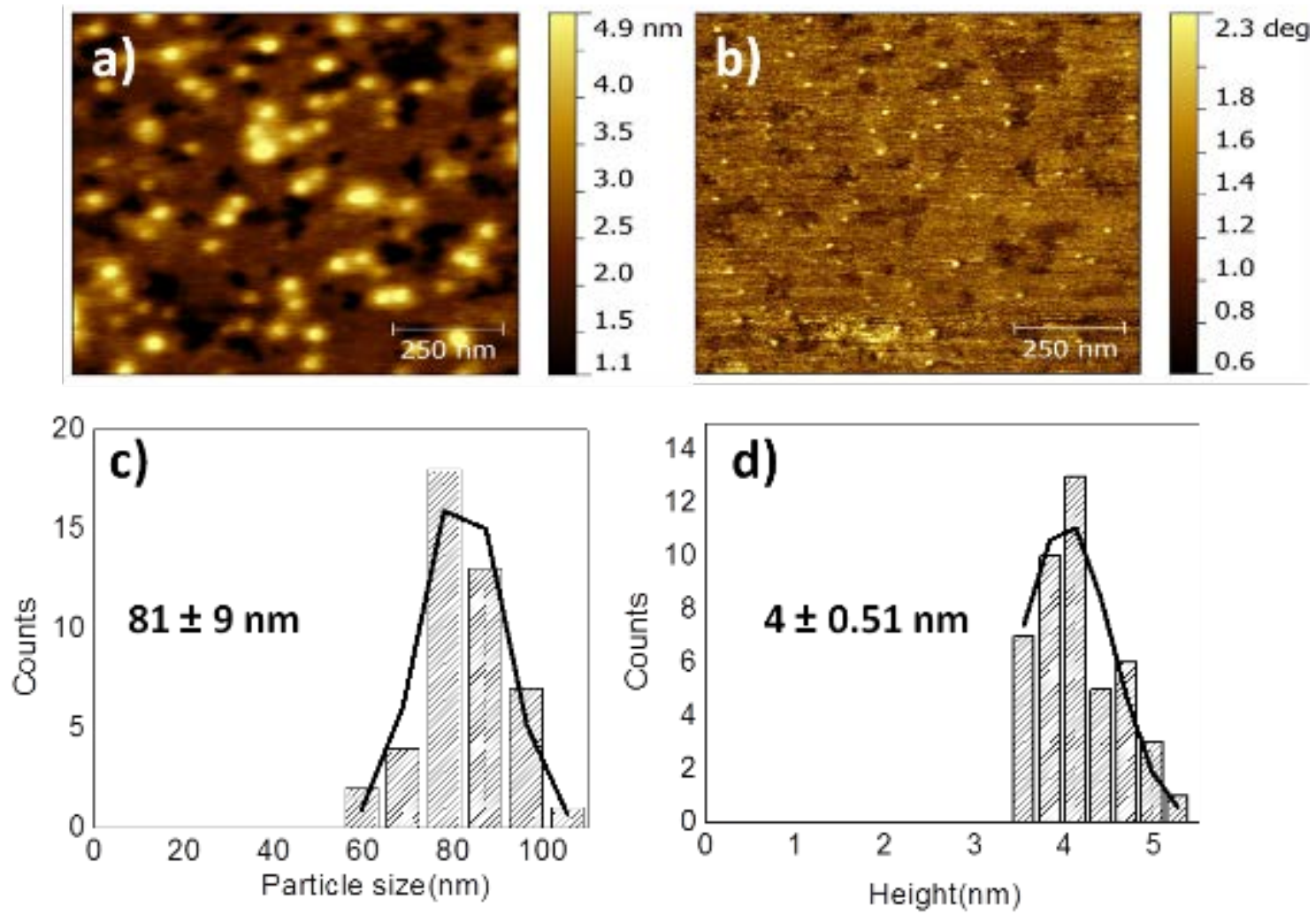

Figure S2.3. Topography (a) and (b) phase images, size distribution (c), and height (d) histograms for control PPE/HA.

2.6.6 Cell Culture: Human Embryonic Kidney (HEK) and human pancreatic cancer (Panc-1) cells were maintained in DMEM (Hyclone, Logan, UT). Human cervical cancer (HeLa) and human breast cancer (MCF7) cells were maintained in MEM/EBSS (Hyclone, Logan, UT). Both media contain 10\% FBS (Hyclone, Logan, UT) and $100 \mathrm{U} / \mathrm{ml}$ penicillin. All cells were incubated in a $5 \% \mathrm{CO}_{2}$ incubator at $37{ }^{\circ} \mathrm{C}$. Cells were seeded in a 12 well-plate and allowed to attach overnight $\left(1 \times 10^{5}\right)$ before incubation with coreshell nanoparticles for the time course experiments.

2.6.7 Cytotoxicity: CellTiter-Glo ${ }^{\circledR}$ Luminescent Cell Viability assay (Promega, Madison, WI) was used to determine the cytotoxicity of the core-shell nanoparticles. HeLa cells $\left(\sim 5 \times 10^{3}\right.$ cells/well) were seeded into a 96 well-plate for 1 day. The cells were then incubated with various concentrations of the core-shell nanoparticle for $24 \mathrm{~h}$. After the core-shell nanoparticle incubation and following the manufacturer's protocol $100 \mu \mathrm{L}$ of CellTiter-Glo reagent was added to each well and the plate was incubated for 2 minutes. Cell viability was monitored by measuring luminescence values of each well using a microplate well reader with 
a 528/20 emission filter (Synergy 2, BioTek, USA). Relative cell viability as a function of the core-shell nanoparticle concentration was obtained by dividing the luminescence value of each sample by the control value. All measurements were triplicated and standard deviation was included in the error bar.

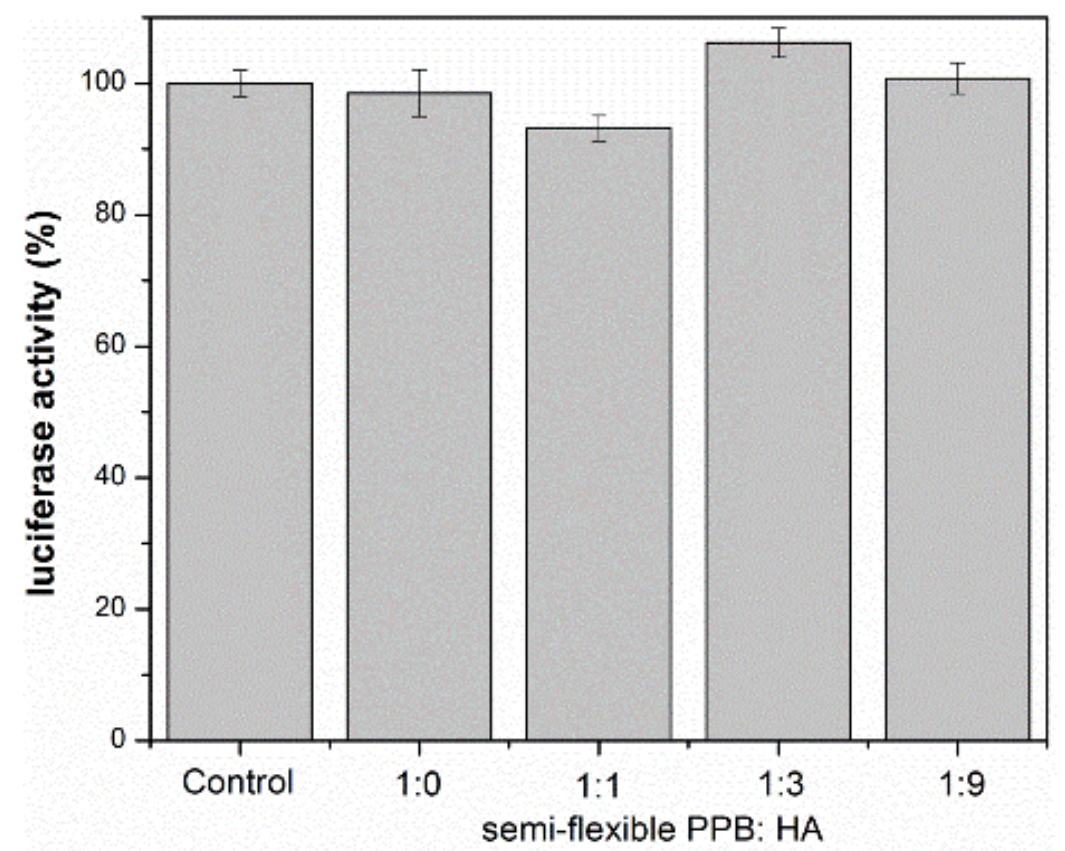

Figure S2.4. Cytotoxicity of the core-shell nanoparticles measured by CellTiter-Glo ${ }^{\circledR}$ Luminescent Cell Viability assay. No toxic effects of were observed under the experimental conditions.

2.6.8 Flow Cytometry: Accuri ${ }^{\mathrm{TM}}$ C6 Flow Cytometer (BD Biosciences, San Jose, CA) equipped with a fluorescence detector was used to analyze the fluorescence intensity of cells incubated with the core-shell nanoparticles. After incubation, cells were rinsed with PBS 2 times followed by centrifugation (Eppendorf Centrifuge $5430 \mathrm{R}$ ) at 1,000 relative centrifugal force (rcf) for $7 \mathrm{~min}$ and resuspension in $400 \mu \mathrm{L}$ FACS buffer. The mean fluorescent intensity of cells treated with the core-shell nanoparticles was normalized to that of untreated control cells. All measurements were triplicated and standard deviation was included in the error bar. For each measurement, 10,000 events were recorded and analyzed.

2.6.9 Evaluation of CD44 expression levels: Cells were seeded in an appropriate medium at a density of 1 x $10^{5}$ cells/ml in a 12-well plate and cultured for overnight. Each cell line was incubated with FITC-labeled mouse anti-human CD44 (BD Pharmingen, San Diego, CA) for $30 \mathrm{~min}$ at $4{ }^{\circ} \mathrm{C}$, according to the protocol provided by the manufacturer. The cells were washed three times with cold PBS and resuspended in $400 \mu \mathrm{L}$ FACS buffer for flow cytometry. 
2.6.10 Microscopic imaging of mixed HeLa and HEK cells: To distinguish HeLa cells co-cultured with HEK cells, HeLa cells were pre-labeled with the CellTracker ${ }^{\mathrm{TM}}$ Red CMPTX (Invitrogen, Grand Island, NY) before co-culturing with HEK cells. $1.75 \mu \mathrm{M}$ of CellTracker Red was incubated with HeLa cells in a 12-well plate $\left(1 \times 10^{5}\right.$ cells/ml) for $30 \mathrm{~min}$ at $37^{\circ} \mathrm{C}$. After replacing the labeling solution with a fresh medium ( 1 $\mathrm{ml}$ ), the cells were further incubated for $30 \mathrm{~min}$ at $37^{\circ} \mathrm{C}$. The HeLa cells labeled with CellTracker were then trypsinized, washed, and re-suspended in $500 \mu \mathrm{L}$ of culture medium. Equal amounts $\left(1 \times 10^{5}\right.$ cells $\left./ \mathrm{ml}\right)$ of both HeLa and HEK cells were mixed in DMEM medium ( $400 \mu \mathrm{L}$ ) containing 10\% fetal bovine serum (FBS) and $100 \mathrm{U} / \mathrm{mL}$ penicillin, and seeded in a glass-bottomed eight-well chamber slide (Lab-Tek, Thermo Scientific). After overnight co-culture, cells were incubated with core-shell nanoparticles (final $10 \mu \mathrm{M}$ ) for $1 \mathrm{~h}$. The cells were rinsed with PBS and were incubated with a Hoechst dye (5 $\mu \mathrm{g} / \mu \mathrm{L})$ (Invitrogen, Grand Island, NY) for 10min. Finally the cells were fixed with 4\% paraformaldehyde in PBS for 10 min at room temperature. Fluorescence images of the cells were obtained using a DeltaVison microscope equipped with 40x lens. Band-pass filters were used for imaging nucleus (410-460 nm), core-shell nanoparticles (500-550 $\mathrm{nm})$, and CellTracker Red (575-620 nm).

\subsection{References}

1. $\quad$ Pinto, M. R.; Schanze, K. S. Proc. Natl. Acad. Sci. U.S.A. 2004, 101, 7505-7510.

2. Gaylord, B. S.; Heeger, A. J.; Bazan, G. C. Proc. Natl. Acad. Sci. U.S.A. 2002, 99, 10954-10957.

3. You, C. C.; Miranda, O. R.; Gider, B.; Ghosh, P. S.; Kim, I. B.; Erdogan, B.; Krovi, S. A.; Bunz, U. H. F.; Rotello, V. M. Nat. Nanotechnol. 2007, 2, 318-323.

4. Wang, L. H.; Pu, K. Y.; Li, J.; Qi, X. Y.; Li, H.; Zhang, H.; Fan, C. H.; Liu, B. Adv. Mater. 2011, 23, 4386-4391.

5. $\quad$ Bajaj, A.; Miranda, O. R.; Kim, I. B.; Phillips, R. L.; Jerry, D. J.; Bunz, U. H. F.; Rotello, V. M. Proc. Natl. Acad. Sci. U.S.A. 2009, 106, 10912-10916.

6. Kim, I. B.; Shin, H.; Garcia, A. J.; Bunz, U. H. F. Bioconjugate Chem. 2007, 18, 815-820.

7. Rahim, N. A. A.; McDaniel, W.; Bardon, K.; Srinivasan, S.; Vickerman, V.; So P. T. C.; Moon, J. H. Adv. Mater. 2009, 21, 3492-3496.

8. Moon, J. H.; McDaniel, W.; MacLean, P.; Hancock, L. E. Angew. Chem. Int. Ed. 2007, 46, 82238225.

9. $\quad$ Yu, J. B.; Wu, C. F.; Zhang, X. J.; Ye, F. M.; Gallina,M. E.; Rong, Y.; Wu, I. C.; Sun, W.; Chan, Y. H.; Chiu, D. T. Adv. Mater. 2012, 24, 3498-3504. 
10. Lee, K.; Lee, J.; Jeong, E. J.; Kronk, A.; Elenitoba-Johnson, K. S. J.; Lim M. S.; Kim, J. Adv. Mater. 2012, 24, 2479-2484.

11. Moon, J. H.; Mendez, E.; Kim, Y.; Kaur, A. Chem. Commun. 2011, 47, 8370-8372.

12. Silva, A. T.; Alien, N.; Ye, C. M.; Verchot, J.; Moon, J. H. BMC Plant Biol. 2010, 10, 291.

13. Feng, X.; Lv, F.; Liu, L.; Yang, Q.; Wang, S.; Bazan, G. C. Adv. Mater. 2012, 24, 5428-5432.

14. Bunz, U. H. F., Acc. Chem. Res. 2001, 34, 998-1010.

15. Roncali, J. Chem. Rev. 1997, 97, 173-205.

16. Thomas, S. W.; Joly, G. D.; Swager, T. M. Chem. Rev. 2007, 107, 1339-1386.

17. Zhu, C. L.; Liu, L. B.; Yang, Q.; Lv, F. T.; Wang, S. Chem. Rev. 2012, 112, 4687-4735.

18. Pecher, J.; Mecking, S. Chem. Rev. 2010, 110, 6260-6279.

19 Tan, S. J.; Jana, N. R.; Gao, S. J.; Patra, P. K.; Ying, J. Y. Chem. Mater. 2010, 22, 2239-2247.

20. Nel, A. E.; Madler, L.; Velegol, D.; Xia, T.; Hoek, E. M. V.; Somasundaran, P.; Klaessig, F.; Castranova, V.; Thompson, M. Nat. Mater. 2009, 8, 543-557.

21. Vokatá, T.; Moon, J. H. Macromolecules 2013, 46, 1253-1259.

22. Ko, Y. J.; Mendez, E.; Moon, J. H. Macromolecules 2011, 44, 5527-5530.

23. Toole, B. P. Nat. Rev. Cancer. 2004, 4, 528-539.

24. Needham, C. J.; Williams, A. K.; Chew, S. A.; Kasper, F. K.; Mikos, A. G. Biomacromolecules 2012, 13, 1429-1437.

25. Goh, E. J.; Kim, K. S.; Kim, Y. R.; Jung, H. S.; Beack, S.; Kong W. H.; Scarcelli, G.; Yun, S. H.; Hahn, S. K. Biomacromolecules 2012, 13, 2554-2561.

26. Choi, K. Y.; Yoon, H. Y.; Kim, J. H.; Bae, M.; Park, R. W.; Kang, Y. M.; Kim, I. S.; Kwon, I. C.; Choi, K.; Jeong, S. Y.; Kim, K.; Park, J. H. ACS Nano 2011, 5, 8591-8599.

27 Li, F.; Bae, B. C.; Na, K. Bioconjugate Chem. 2010, 21, 1312-1320.

28. Choi, K. Y.; Chung, H.; Min, K. H.; Yoon, H. Y.; Kim, K.; Park, J. H.; Kwon I. C.; Jeong, S. Y. Biomaterials 2010, 31, 106-114.

29. Tang, Y. L.; Hill, E. H.; Zhou, Z. J.; Evans, D. G.; Schanze, K. S.; Whitten, D. G. Langmuir 2011, 27, 4945-4955.

30. Tang, Y.; Zhou, Z.; Ogawa, K.; Lopez, G. P.; Schanze, K. S.; Whitten D. G. Langmuir 2009, 25 , 21-25.

31. Kim, J.; Swager, T. M. Nature 2001, 411, 1030-1034.

32. Mendez, E.; Moon, J. H. Chem. Comm. 2013, 49, 6048-6050. 


\section{CHAPTER III}

Differential interactions of conjugated polymer nanoparticles with glycosaminoglycans in synthetic urine

Reproduced in part with permission from Chemical Communications 2015, 51, 4065-4068

Copyright $@ 2015$

Royal Society of Chemistry

Megan Twomey, Tereza Vokatá, Manian Rajesh Kumar, and Joong Ho Moon*

Department of Chemistry \& Biochemistry, Florida International University, Miami, Florida 33199, United States

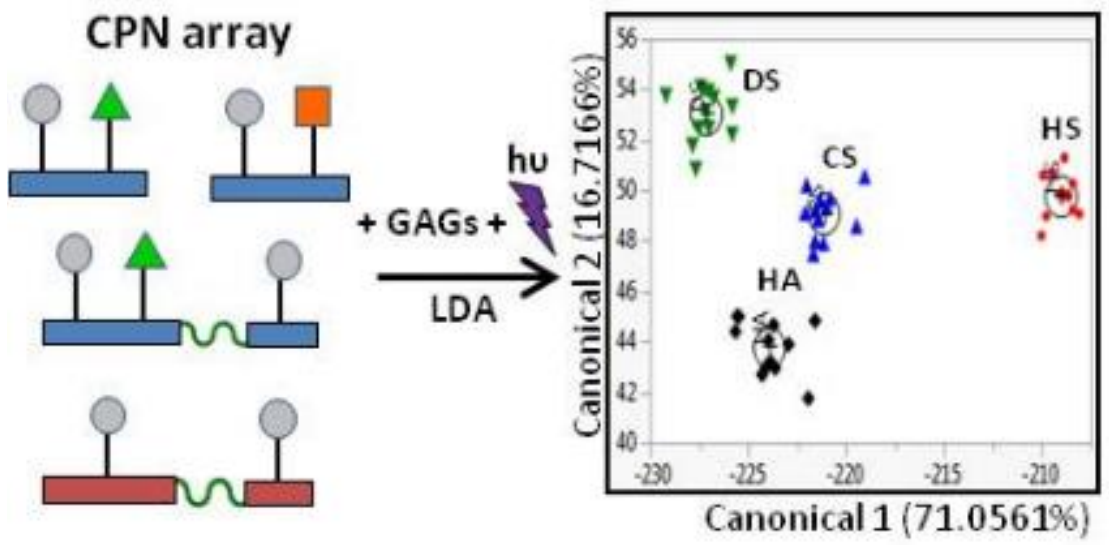




\subsection{Abstract}

Four different conjugated polymer nanoparticles (CPNs) were used to differentiate structurally similar glycosaminoglycans (GAGs) in a urine simulant. Unique emission response patterns of CPNs were analyzed by linear discriminant analysis (LDA), confirming that structurally diverse CPNs are sensitive and effective at differentiating GAGs in a complex biological medium.

\subsection{Introduction}

Changes in urinary glycosaminoglycan (GAG) levels can signify proliferation of several diseases including kidney and bladder disorders, polysaccharide storage diseases, and certain cancers. ${ }^{1-4}$ However, detection or differentiation of GAGs in a biological medium has been challenged by the structural similarity of GAGs and interferences of the concomitant biomolecules commonly present in biological fluids. Conventional GAG detection methods require sample preparation steps followed by complicated instrumental analysis or biochemical assays. ${ }^{5-9}$ Recently, several researchers have developed conceptually important detection methods on the basis of pattern recognition using nanoparticles, conjugated polyelectrolytes, and liposomes. ${ }^{10-14}$ In those examples, differentiation of GAGs was demonstrated by using multivariate statistical methods including linear discriminant analysis (LDA). While current sensory systems perform well in a simplified solution (i.e., highly diluted media with buffers), few sensory systems have been reported for sensitive detection of GAGs in complicated biological fluids. Development of simple and sensitive sensory systems of GAGs in biological media is highly important and practical for future disease diagnostics.

Owing to their excellent photophysical properties, conjugated polymers (CPs) have attracted much attention for optical detection of chemicals, metal ions, and biological substances. ${ }^{15-18}$ Many synthetic and fabrication methods have been developed to increase aqueous compatibility of CPs to achieve necessary sensitivity for specific analytes in aqueous environments. ${ }^{19}$ Depending on the aqueous solubility of CPs and the nature of interaction between CPs and analytes, structural changes can occur in individual CP chains or multiple chain aggregates, which correspond to changes in CP optical properties. ${ }^{20,21}$

Previously, positively charged conjugated polymer nanoparticles (CPNs) were fabricated by treating a nonaqueous soluble, primary amine-containing CP [i.e., poly(p-phenyleneethynylene) (PPE)] with organic acids followed by dialysis. ${ }^{22}$ The aggregation structures and sizes of CPNs were dependent on the nature of organic 
acid treatment. Furthermore, it was found that CPNs fabricated with a semi-flexible CP [i.e., a flexible linker containing poly(p-phenylenebutadiynylene) (PPB)] exhibited backbone reorganization to maximize hydrophobic chain interaction when treated with an anionic linear polysaccharide, hyaluronic acid (HA). ${ }^{23,24}$ The structural reorganization of CPNs was evident by photophysical changes including a new sharp absorption peak at longer wavelength and decreased fluorescence intensity. The physical change was observed as an elongated particle shape shown in atomic force microscopic images.

From these observations, it was hypothesized that the aggregation status of cationic CPNs will be different upon interaction with GAGs due to the differences in ionic strength of the GAGs exhibiting different degrees of acetylation and sulfonation in the repeating disaccharide units (see Figure 3.1). While the hydrophobic interaction among non-aqueous soluble CPs provides the structural integrity of CPNs in a biological medium, the loosely aggregated CPNs will undergo backbone reorganization under polyelectrolyte interactions with GAGs. Depending on the strength of ionic interactions, the aggregation properties of CPNs will change accordingly, resulting in measurable spectral changes.

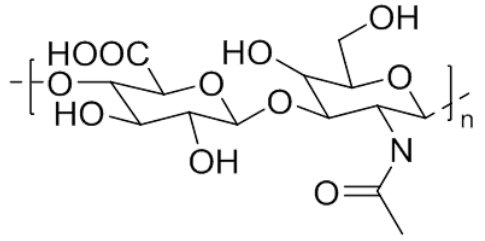

Hyaluronic acid (HA)

Avg. $\mathrm{MW}(\mathrm{KDa})=100$

Avg. charge $=-1$

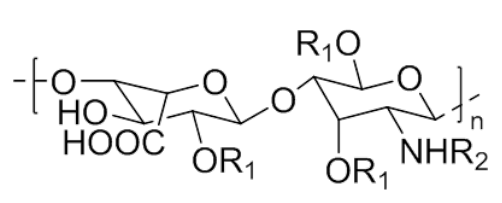

Heparin sulfate (HS)

Avg. $\mathrm{MW}(\mathrm{KDa})=100$

Avg. charge $=-3.7$

$$
\mathbf{R}_{\mathbf{1}}=\mathrm{H}, \mathrm{SO}_{3}^{-} \quad \mathbf{R}_{\mathbf{2}}=\mathrm{H}, \mathrm{SO}_{3}^{-}, \mathrm{COCH}_{3}
$$

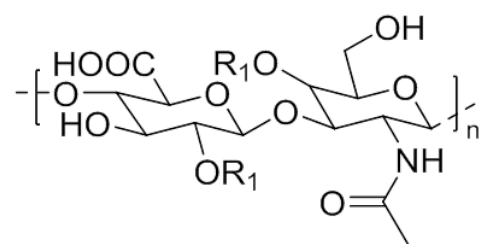

Chondroitin sulfate (CS)

Avg. $\mathrm{MW}(\mathrm{KDa})=20$

Avg. charge $=-2$

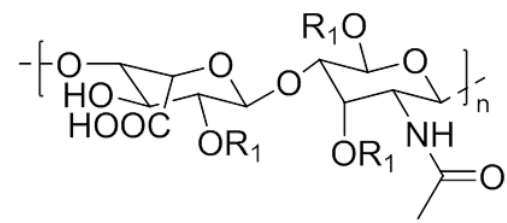

Dermatan sulfate (DS)

Avg. $\mathrm{MW}(\mathrm{KDa})=25$

Avg. charge $=-3$

Figure 3.1. Chemical structures of glycosaminoglycans.

In this chapter, four different CPNs were used that act as both analyte receptors and signal transducers, and analyzed their differential responses to each GAG in a urine simulant. A systematic investigation of the 
aggregation properties of CPNs that vary by side chain amine density, backbone flexibility, and type of backbone structure in response to GAGs was conducted by monitoring changes in absorption/emission profiles and size/size distributions. Finally, entire spectral responses of the structurally diverse CPNs were analyzed by LDA to differentiate structurally similar GAGs at a physiologically relevant concentration ${ }^{25}$ in a urine simulant. Side chain and backbone flexibility strongly affects both the physical and photophysical properties of CPN/GAG complexes. A clear differentiation of recognition patterns was observed in a LDA plot, supporting that structurally diverse CPNs are effective at differentiating GAGs in a complex biological medium.

\subsection{Results and Discussion}

Four CPs having different side chain and backbone structures (Figure 3.2) were used to obtain an array of CPNs with different aggregation natures. P1 was designed to compare a short ethylene oxide (EO) and primary amine [after removal of N-tert-butoxycarbonyl (Boc) group] per repeating unit, to the side chains of P2 with a higher amine density by replacing the EO side chain with a guanidinium group. P3 was designed to increase backbone flexibility by introducing a non-conjugated, flexible moiety in the conjugated phenyleneethynylene (PE) backbone, while maintaining the fluorescent nature and the side chain functionality of P1. Lastly, P4 was used because the flexible phenylenebutadiynylene (PB) backbone induces higher backbone aggregation than the structurally similar PE analogues.
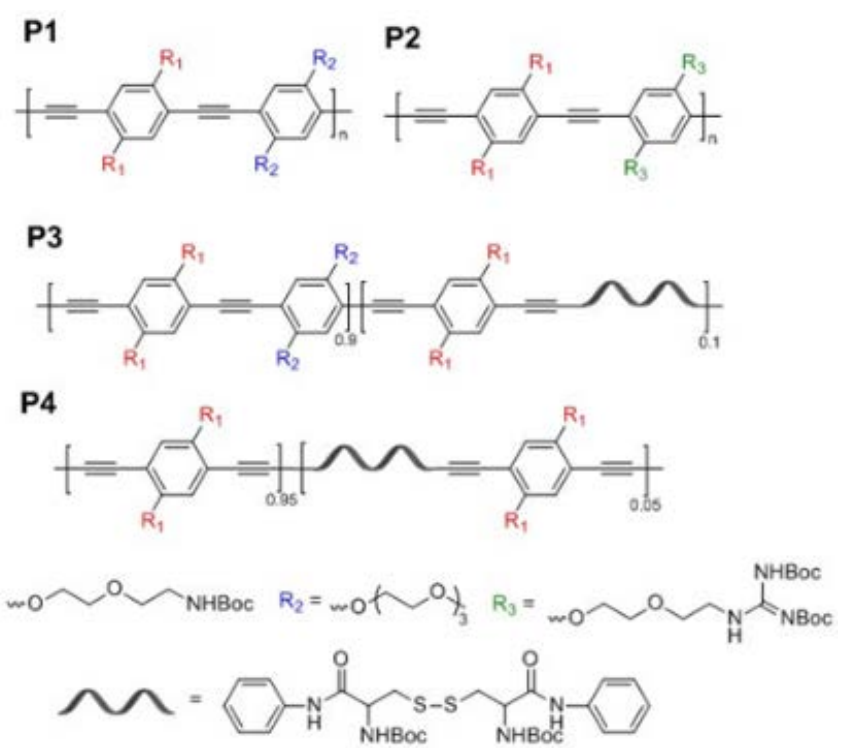

Figure 3.2. Chemical structures of CPs with different side chains (P1 and P2), PPE with flexible backbone (P3), and PPB with flexible backbone (P4). 
The cationic CPNs were fabricated using our previously published method, ${ }^{23}$ in which the corresponding P1P4 were treated with trifluoroacetic and acetic acid followed by dialysis. The resulting nanoparticles in water were homogeneous yellow solutions, and the key characteristic properties are summarized in Table 3.1.

Table 3.1. Key photophysical and physical properties of CPNs.

\begin{tabular}{|c|c|c|c|c|c|c|}
\hline CPN & $\mathrm{CP}$ & $\begin{array}{l}\mathrm{M}_{\mathrm{n}}^{\mathrm{a}} \\
(\mathrm{kDa})\end{array}$ & $\begin{array}{c}\lambda_{\max , \text { abs }}{ }^{\mathrm{b}} \\
(\mathrm{nm})\end{array}$ & $\begin{array}{c}\lambda_{\max , \mathrm{em}^{\mathrm{c}}} \\
(\mathrm{nm})\end{array}$ & $\begin{array}{l}\text { Hydrodynamic } \\
\text { diameter }^{\mathrm{d}}(\mathrm{nm})\end{array}$ & $\begin{array}{l}\text { Zeta potential } \\
(\mathrm{mV})\end{array}$ \\
\hline 1 & P1 & 17,457 & 417 & 482 & $140 \pm 0.9$ & $+40 \pm 1.3$ \\
\hline 2 & $\mathbf{P 2}$ & 14,079 & 411 & 479 & $179 \pm 6.3$ & $+54 \pm 3.1$ \\
\hline 3 & P3 & 14,489 & 422 & 464 & $117 \pm 10.1$ & $+48 \pm 2.9$ \\
\hline 4 & P4 & 35,907 & 444 & 466 & $134 \pm 8.1$ & $+30 \pm 1.7$ \\
\hline
\end{tabular}

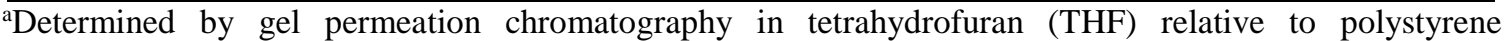
standard. ${ }^{b}$ Measured in water. ${ }^{~}$ Excited at $400 \mathrm{~nm}$. ${ }^{\mathrm{d}}$ Measured by single particle tracking analysis at $25^{\circ} \mathrm{C}$ in

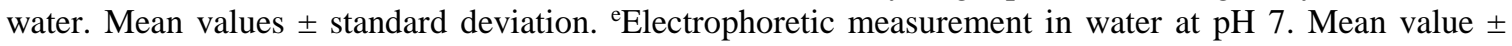
standard deviation.

The hydrodynamic diameters of CPNs were measured using nanoparticle tracking analysis (NTA), which tracks the Brownian motion of each particle to accurately calculate the hydrodynamic diameter of particles with size distributions. ${ }^{26}$ The hydrodynamic diameters of CPNs ranged from $\sim 120$ to $180 \mathrm{~nm}$ in water. The relatively larger diameter of $\mathrm{CPN}-2$ can be attributed to the hydrophilic nature of side chains, which form aggregates of more solvated chains. Upon complexation, diameters of CPNs changed as aggregation properties of CPNs were affected by GAGs with different ionic strengths. As shown in Figure 3.3, CPN-2 showed decreased sizes upon complexation, while the rest of the CPNs exhibited increased sizes. The loosely aggregated, more solvated P2 chains are believed to form compact and smaller complexes with GAGs (see Figure 3.4). Increased sizes observed from the rest of the CPNs were likely due to the formation of hydrophilic GAG shells on CPNs. Among them, CPN-4 showed the largest diameter increase, confirming that the ionic interaction induces further CP chain aggregation due to the nature of PPB backbone with flexibility. A complete summary of NTA results are given in Table S3.1. 


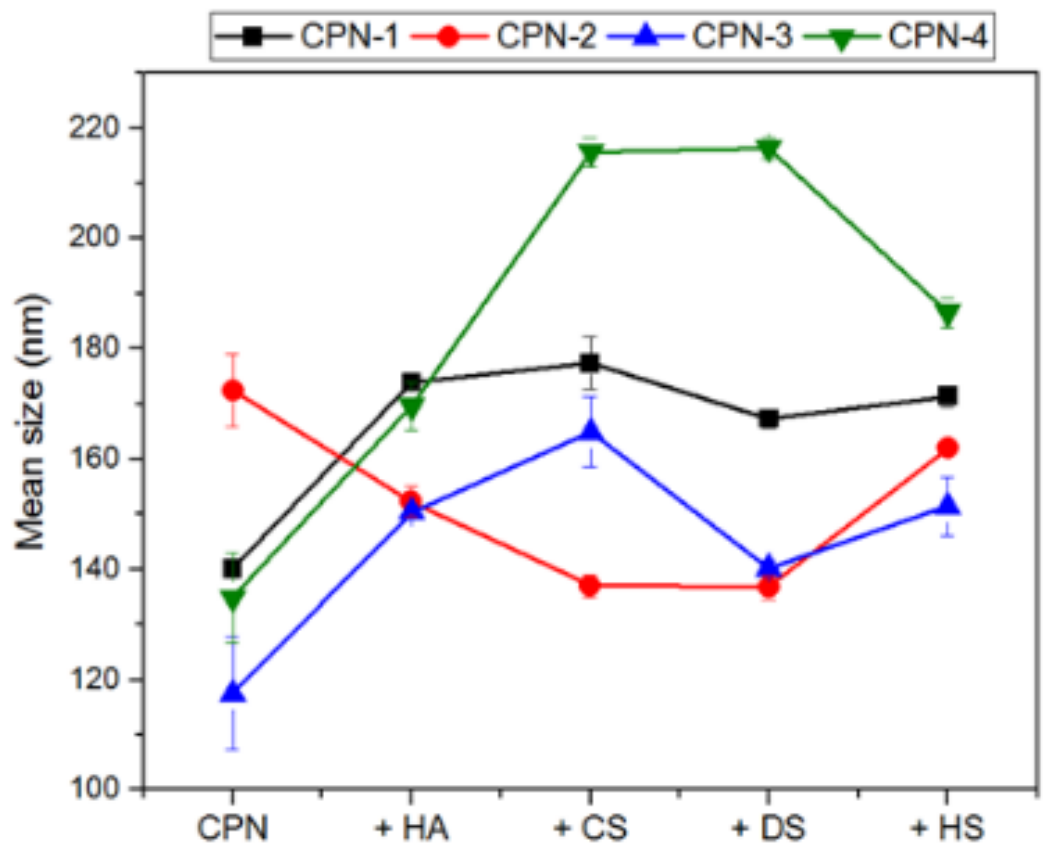

Figure 3.3. Average hydrodynamic diameters of CPN-1 (black square), CPN-2 (red circle), CPN-3 (blue triangle point up), and CPN-4 (green triangle point down) upon complexation with hyaluronic acid (HA), chondroitin sulfate (CS), dermatan sulfate (DS), and heparin sulfate (HS), respectively, measured by NTA in triplicate.

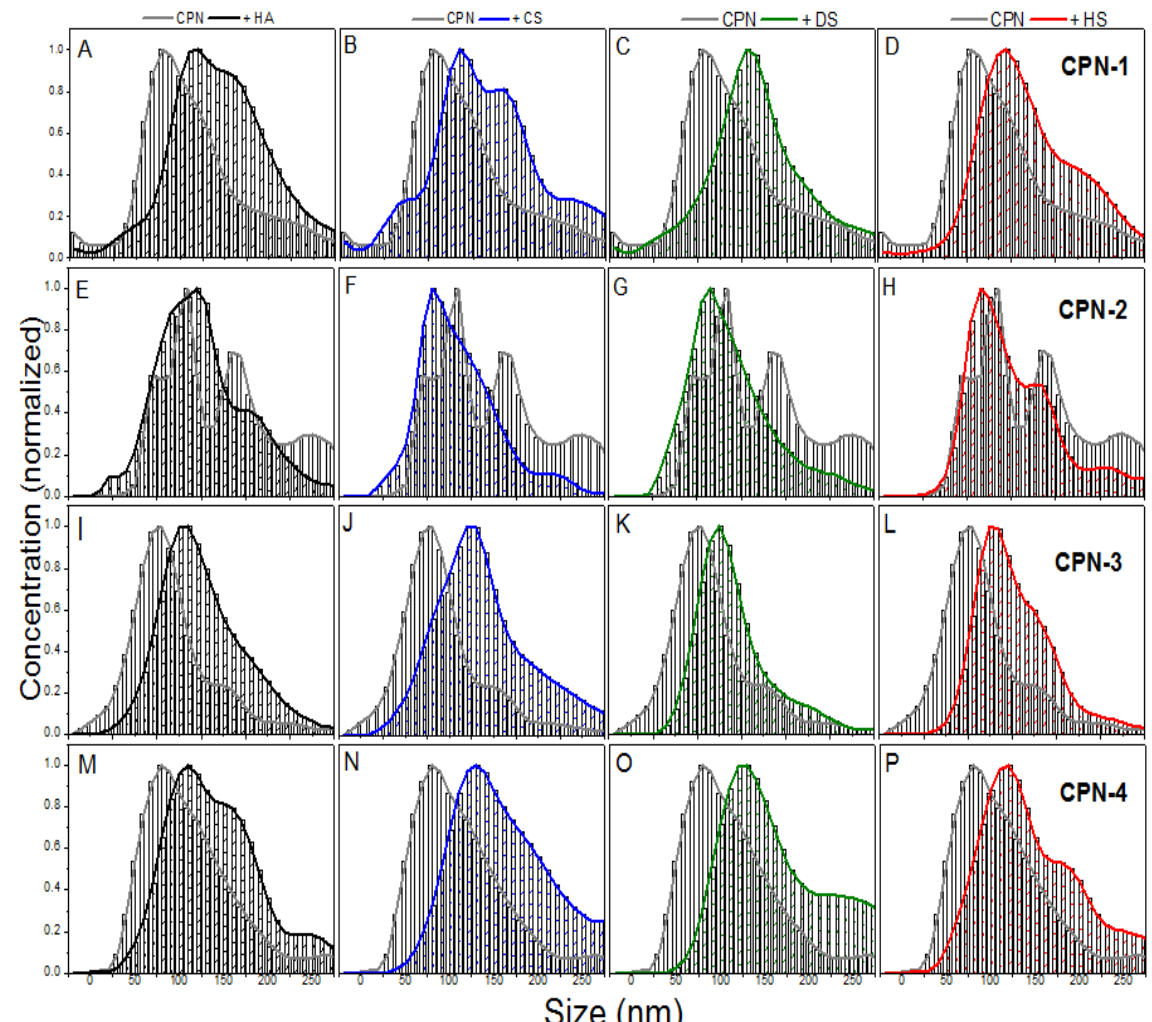

Figure 3.4. Average size distributions of CPNs complexed with GAGs measured by NTA. 
The absorption and fluorescent profiles support the NTA data. GAGs induce changes in the aggregation structures as evidenced by red shifts in absorption and decreased fluorescent intensity in emission spectra for all CPNs (Figure 3.5). As expected, CPN-4 displayed dramatic changes in both absorption and emission profiles due to extensive chain reorganization. CPN-3, which also has flexible linkers along the PPE backbone, displayed relatively small changes in the absorption, implying that the chemical backbone structure of the $\mathrm{CP}$ is an important contributor for chain reorganization. The structurally similar GAGs also interact differently with CPNs with structural diversity. The carboxylated HA induced higher absorption shifts compared to the sulfonated GAGs, although the effects were somewhat minimal, with the exception of CPN-4. Due to the relatively weak ionic strength of carboxylic acid, compared to sulfonic acid, HA is believed to form outer shell layers by contributing to increase $\pi-\pi$ interaction among CPs. Meanwhile, GAGs with sulfonic acids interact strongly with CPs, especially with CP chains of low molecular weights, resulting in more solvated random complexes. As expected, the emission profiles of all CPNs were unique and different upon complexation with GAGs; These spectral changes can then be used for differentiation of GAGs. Hierarchical cluster analysis (HCA) of emission profiles show that the sulfonated GAGs cluster together, indicating that there is differentiation based on functional group (see Figure 3.6). For simplicity, one CPN emission data set in water (triplicate samples) was used for HCA to demonstrate role of functional group in differentiation. The most structurally similar chondroitin sulfate (CS) and dermatan sulfate (DS) form the closest cluster, and the most structurally different hyaluronic acid (HA) and heparin sulfate (HS) form the furthest cluster. 

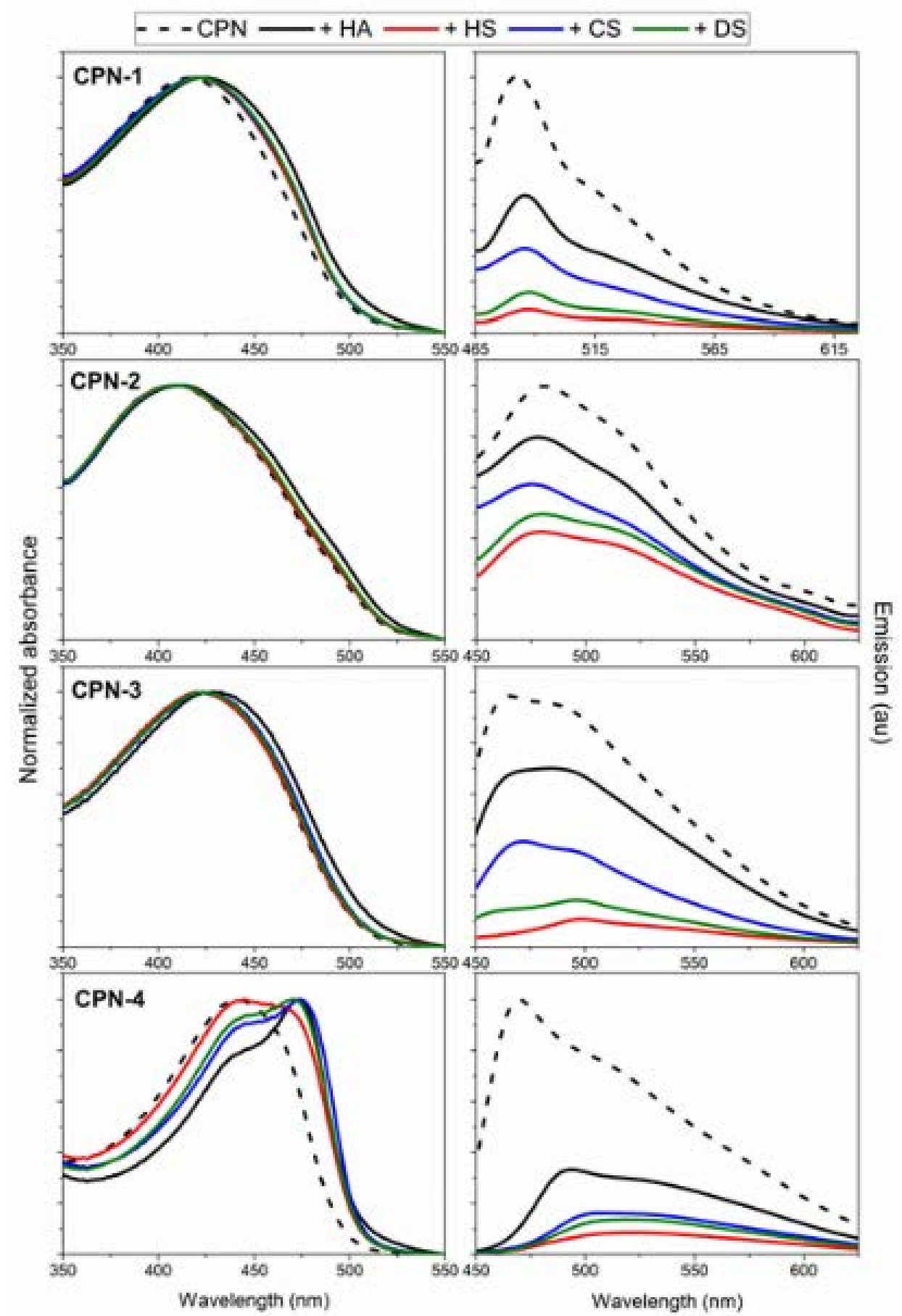

Figure 3.5. Effect of GAG complexation on absorption (left column) and emission (right column) for CPN1 (first row), CPN-2 (second row), CPN-3 (third row), and CPN-4 (fourth row) in water. Optical density of CPNs was fixed at 0.1 . 


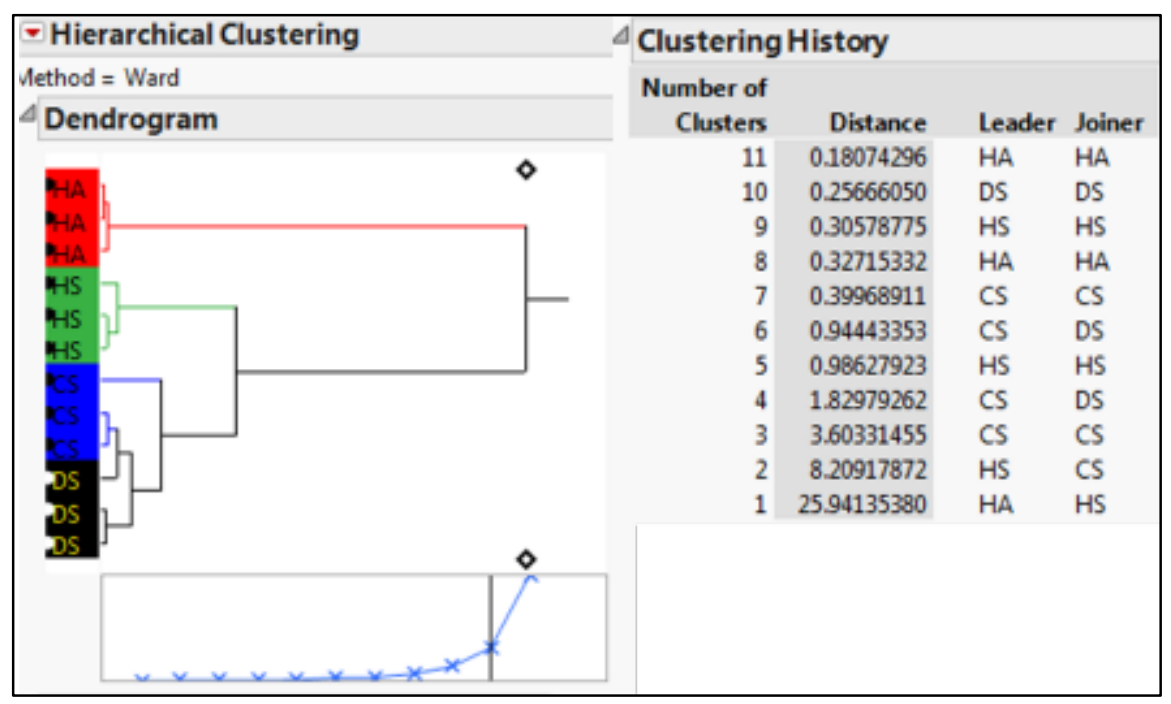

Figure 3.6. Hierarchical cluster analysis (HCA) of CPN-2+GAGs in water.

The differentiation ability of CPNs in a complex aqueous medium was verified using LDA of fluorescence spectral responses of CPNs to GAGs. This method can be used to establish a pattern for a "chemical nose" type array sensor, ${ }^{27}$ which relies on a differential response of the receptor (i.e., CPN) to the analyte (i.e., GAG). After adding CPNs to the GAG solution (100 nM) in a commercially available urine simulant, emission spectra (see Figure 3.7) of complexes were recorded and used for LDA. The ratios of fluorescent intensity were analysed using the statistical software JMP® (version 11). The LDA plot depicting the first two canonicals is shown in Figure 3.8. A summary of canonical scores can be found in Table S3.2. There is a clear separation among the GAGs, with no overlap of groups with a 95\% confidence limit. Three canonical correlations account for $71.1,16.7$, and $12.2 \%$ of the variation, occupying $100 \%$ of the total variation between the groups, indicating that GAG differentiation is maximized. Traditional discriminant functions correctly predict $100 \%$ of group classification, based on their squared distances to each group centroid (Table S3.3). These results confirm that loosely aggregated CPNs can undergo structural reorganization in the presence of strong polyelectrolytes in a complex biological medium due to the hydrophobic CP backbone that provides the necessary structural integrity to resist interference from environmental factors. 

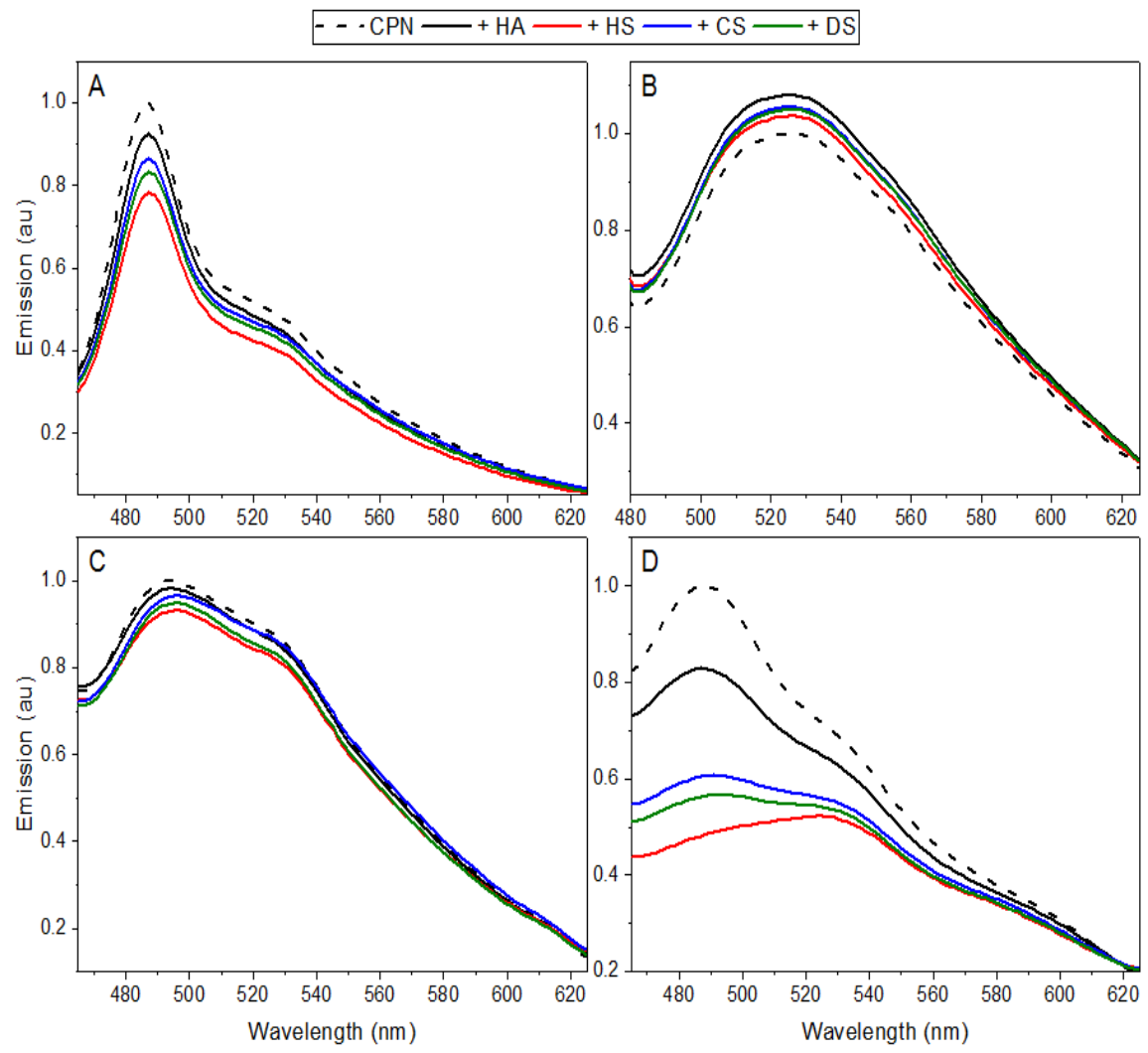

Figure 3.7. Emission spectra of CPN-1 (a), CPN-2 (b), CPN-3 (c), and CPN-4 (d) in the presence of GAGcontaining urine simulant. Excitation wavelength for all CPNs was $450 \mathrm{~nm}$ with $5 \mathrm{~nm}$ slit widths, and $0.5 \mathrm{~s}$ integration time.

\section{HA HS $\Delta$ CS $\nabla$ DS}

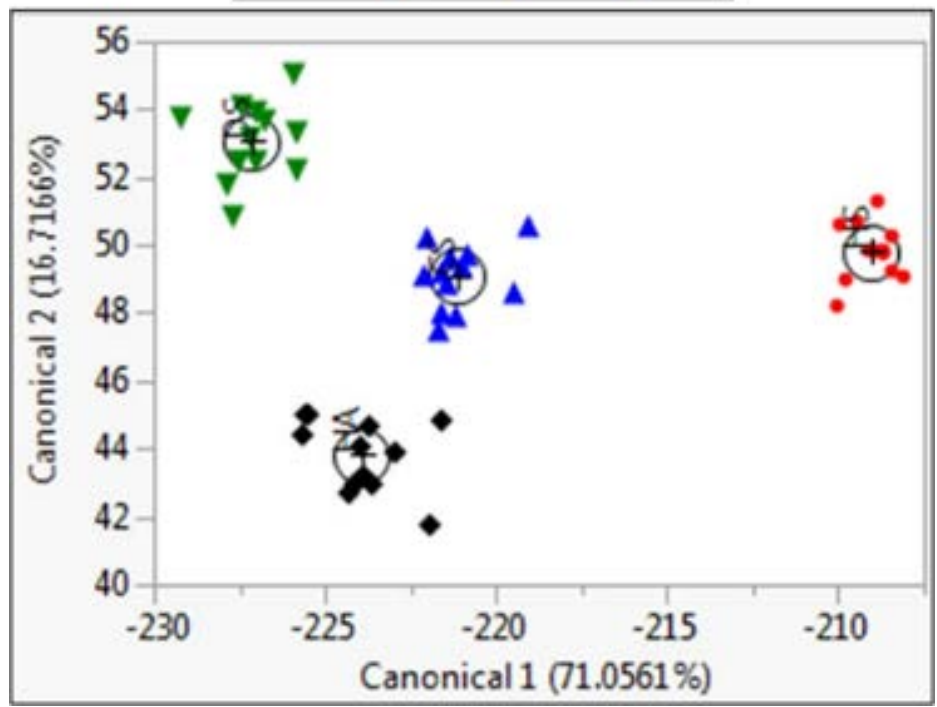

Figure 3.8. An LDA plot between two largest canonical correlations of emission intensity ratios of CPNs with and without GAGs $(100 \mathrm{nM})$ in a urine simulant. HA in black, HS in red, CS in blue, and DS in green, with $(+)$ marking the centroid of each group. 


\subsection{Conclusion}

In summary, a systematic investigation is reported on the aggregation properties of CPNs that vary by side chain and backbone structures in response to GAGs by observing changes in absorption/emission profiles and complex size/size distributions. This investigation demonstrated that side chain and backbone flexibility strongly affect the photophysical and physical properties of CPN/GAG complex. Four CPNs and their differential responses to each GAG were analyzed using the LDA method to demonstrate that structurally diverse CPNs differentiate GAGs in a complex biological medium. The structure-function relationships obtained from this work will lead to further improvements in designing functional polymers for sensing biological and biomedical substances.

\subsection{Outlook}

This chapter presented a simple approach to differential sensing of biologically important molecules. A systematic approach to understanding the effect of side chain and backbone structure was employed to establish a structure-function relationship. To improve differential sensing, an array of more structurally diverse CPNs could be utilized, which would lead to more dramatic differential interactions of CPNs and analytes. Since publication of this work in Chemical Communications, controlled incorporation of homocoupled benzothiadiazole (HBT) blocks into the conjugated polymer backbone has been explored. The HBT blocks provide an electron-accepting moiety in the backbone, which modulates the CP's electronic properties towards red emission. By developing a more structural diverse array, the sensitivity can be greatly improved. The unique signal amplification of CPNs, where any interaction that occurs along the conjugated backbone causes a perturbation in the electron transport that will affect the entire system, makes CPN-based sensors an ideal platform for improving interactions with analytes (more in section 4.5). In addition, a more systematic investigation of CPN structure was conducted to understand the polyanion complexation behavior. ${ }^{29} \mathrm{~A}$ series of four conjugated polyelectrolytes (CPEs) with PPE and PPB-type connectivity and incorporation of a flexible, non-conjugated linker were prepared and displayed backbone-dependent complexation with HA. Cellular uptake and subcellular localization studies of these CPEs would provide more information how exactly backbone connectivity affects cellular interactions. 


\subsection{Experimental}

\subsubsection{General.}

Chemicals and solvents were purchased from Fisher Scientific and used as received. Deuterated solvents were purchased from Cambridge Isotope Laboratories (Cambridge, MA). Molecular weights were determined using gel permeation chromatography (GPC) against polystyrene standards on a Shimadzu high performance liquid chromatography (HPLC) system equipped with PLgel $5 \mu \mathrm{m}$ MIXED-D columns and SPD-20A ultraviolet-visible (UV-vis) detector a flow rate of $1.0 \mathrm{~mL} / \mathrm{min}$. UV-Vis spectra were recorded using Varian Cary 50 Bio spectrophotometer. Fluorescence spectra were obtained using a FluoroLog-3 Spectrofluorometer (Jobin Yvon/Horiba). Quantum yields (QYs) were determined using 9,10-diphenylanthracene $(\mathrm{QY}=0.9)$ in cyclohexane as a fluorescence standard. Fourier transform infrared (FT-IR) spectra were recorded on a PerkinElmer Spectrum 100 FT-IR Spectrometer. Fine powders from lyophilized samples were directly mounted on an attenuated total reflection (ATR) cell of the spectrometer. The purification of the CPNs was conducted using an Ultrafiltration Stirred Cell (Millipore) with a $10 \mathrm{kDa}$ molecular weight cut-off (MWCO) membrane (Ultracel ultrafiltration disc). Nuclear magnetic resonance (NMR) spectra for the conjugated polymers (CPs) were recorded on a $400 \mathrm{MHz}$ Avance Bruker NMR spectrometer. Chemical shifts were reported in parts per million (ppm) for ${ }^{1} \mathrm{H}$ NMR on the $\delta$ scale based on the middle peak $\left(\delta=2.50 \mathrm{ppm}\right.$ ) of the dimethylsulfoxide (DMSO)- $\mathrm{d}_{6}$ solvent as an internal standard. NMR spectra for the conjugated polymer nanoparticles (CPNs) were recorded on a $600 \mathrm{MHz}$ Avance Bruker NMR spectrometer using a $5 \mathrm{~mm}$ BBI probe at $298 \mathrm{~K}$. The $600 \mathrm{MHz}$ NMR spectrometer is equipped with a gradient system capable of producing magnetic field pulse gradients in the z-direction of about $50 \mathrm{G} \mathrm{cm}^{-1}$ and allowing for water peak suppression $\left[\delta=4.79 \mathrm{ppm}\right.$ in deuterium oxide $\left.\left(\mathrm{D}_{2} \mathrm{O}\right)\right]$. Chemical shifts were reported in parts per million (ppm) for ${ }^{1} \mathrm{H}$ NMR on the $\delta$ scale based on the middle peak $(\delta=4.79 \mathrm{ppm})$ of $\mathrm{D}_{2} \mathrm{O}$ solvent as an internal standard. Graphs were plotted using Origin 9.1 software (OriginLab, Northampton, MA, USA).

\subsubsection{Polymer synthesis general procedure.}

A Schlenk flask was charged with aryl halide monomer (1.0 equiv) and diacetylene monomer (1.0 equiv for P2, 0.9 equiv for P3), and cystine linker when applicable (0.1 equiv for P3) along with $\operatorname{Pd}\left[\left(\mathrm{PPh}_{3}\right)_{2} \mathrm{Cl}_{2}\right](0.1$ equiv) and CuI (0.05 equiv). The Schlenk flask was evacuated and filled with $\mathrm{N}_{2}$ three times. A solution of 
anhydrous dimethylformamide (DMF) (3 mL) and morpholine $(1 \mathrm{~mL})$ was degassed, and $2 \mathrm{~mL}$ of the mixed solution was transferred to the Schlenk flask using a cannular needle. The reaction mixture was heated at 50 ${ }^{\circ} \mathrm{C}$ for $18 \mathrm{~h}$. The solution was then cooled to room temperature and transferred dropwise to cold ethyl ether, resulting in precipitation. After centrifugation (5 min, $4000 \mathrm{rpm}$ ), the supernatant was decanted, and the precipitate was redissolved in DMF (1 mL). The resulting polymer was characterized using GPC by diluting an aliquot of polymer solution in $1 \mathrm{~mL}$ of HPLC grade tetrahydrofuran (THF) and filtered through $0.45 \mu \mathrm{m}$ polytetrafluoroethylene (PTFE) syringe filter prior to injection. The absorption and emission profiles were measured in a $10 \mathrm{~mm}$ quartz cuvette $(2 \mathrm{~mL})$ using a diluted aliquot of the polymer solution in DMF. The material was then reprecipitated in pure ether, the supernatant was decanted, and the precipitate was purified two more times. The precipitated polymer was allowed to dry under high vacuum for 4 hours prior to FT-IR and ${ }^{1} \mathrm{H}$ NMR characterization.

\subsubsection{CPN fabrication.}

Boc-deprotection of the polymer was carried out by adding the polymer solution in DMSO- $\mathrm{d}_{6}$ to a stirred mixture of trifluoroacetic acid (2 mL) and acetic acid $(2 \mathrm{~mL})$ and allowed to stir at room temperature for 2 days. The mixture was then diluted by addition of acetic acid (10 mL), and added dropwise (2 drops/s) to 500 $\mathrm{mL}$ water (18 $\Omega$ ) while stirring. Using a solvent-resistant stir cell fitted with a $10 \mathrm{kDa}-\mathrm{MWCO}$ membrane, the solution was concentrated to approximately $10 \mathrm{~mL}$, and dialyzed against $2 \mathrm{~L}$ of water. The solution was subsequently filtered through a cellulose syringe filter $(0.45 \mu \mathrm{m})$, characterized, and stored for future use.

P1: Detailed monomer and polymer synthesis, CPN fabrication and characterization is described elsewhere. ${ }^{28}$

P2: Detailed monomer synthesis and characterization of monomers $\mathbf{A}$ and $\mathbf{B 1}$ is described elsewhere. ${ }^{28,29}$ Using the general procedure described above, the polymerization of monomer A (7.8 $\mathrm{mg}, 0.0147 \mathrm{mmol})$ and monomer B1 (15.0 mg, $0.0147 \mathrm{mmol})$ in the presence of $\mathrm{Pd}\left[\left(\mathrm{PPh}_{3}\right)_{2} \mathrm{Cl}_{2}\right](1.4 \mathrm{mg}, 0.00147 \mathrm{mmol})$ and $\mathrm{CuI}$ (0.1 mg, $0.000735 \mathrm{mmol}$ ) yielded P2 (11.5 mg, $0.00866 \mathrm{mmol}$, 58.9\%), see scheme S3.1. CPN fabrication was carried out as described in the general procedure to yield CPN-2, see scheme S3.2. 
Scheme S3.1. Synthetic route to P2.

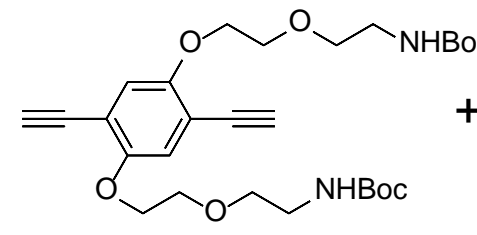

A

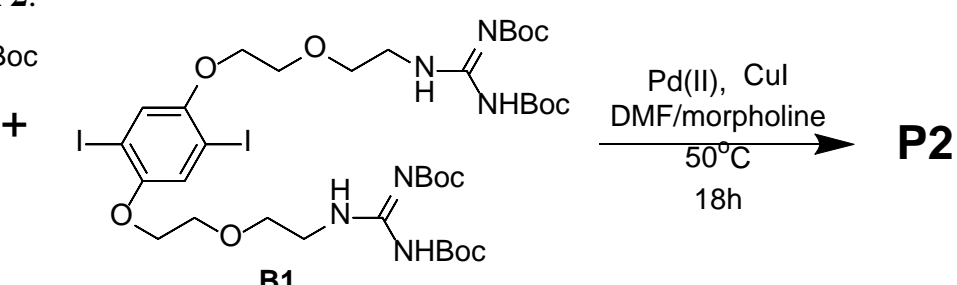

B1

P2: ${ }^{1} \mathrm{H}$ NMR (400 MHz, DMSO-d 6 ): $\delta 13.19$ (s, 0.15H), 12.38 (s, 1.18H), 9.00 (s, 0.15H), 8.19 (s, $1.16 \mathrm{H}$ ), 7.11 (d, 2.07H), 6.70 (s, 1.23), 4.17 (br m, 4.39H), 3.85-3.76 (br m, 7.09H), 3.56-3.45 (br m, 13.5 H), 3.08 (s, 2.88H), 1.44-1.34 (d, 27.0 H). FT-IR (neat): 3351, 2973, 2965, 2928, 2256, 1709, 1630, 1583, 1505, $1455,1411,1366,1301,1273,1245,1146,1113,1050,1023 \mathrm{~cm}^{-1}$. GPC: $\mathrm{M}_{\mathrm{w}}=23,618 \mathrm{Da}, \mathrm{M}_{\mathrm{n}}=14,079 \mathrm{Da}$, $\mathrm{PDI}=1.68 . \mathrm{UV}-\mathrm{Vis}(\mathrm{DMF}) \lambda_{\max }=439 \mathrm{~nm}$, fluo $\lambda_{\max }(430 \mathrm{~nm}$ ex $)=475 \mathrm{~nm}, \mathrm{QY}=20 \%$.

Scheme S3.2. Boc-deprotection of $\mathbf{P 2}$ to yield $\mathbf{C P N}-2$.
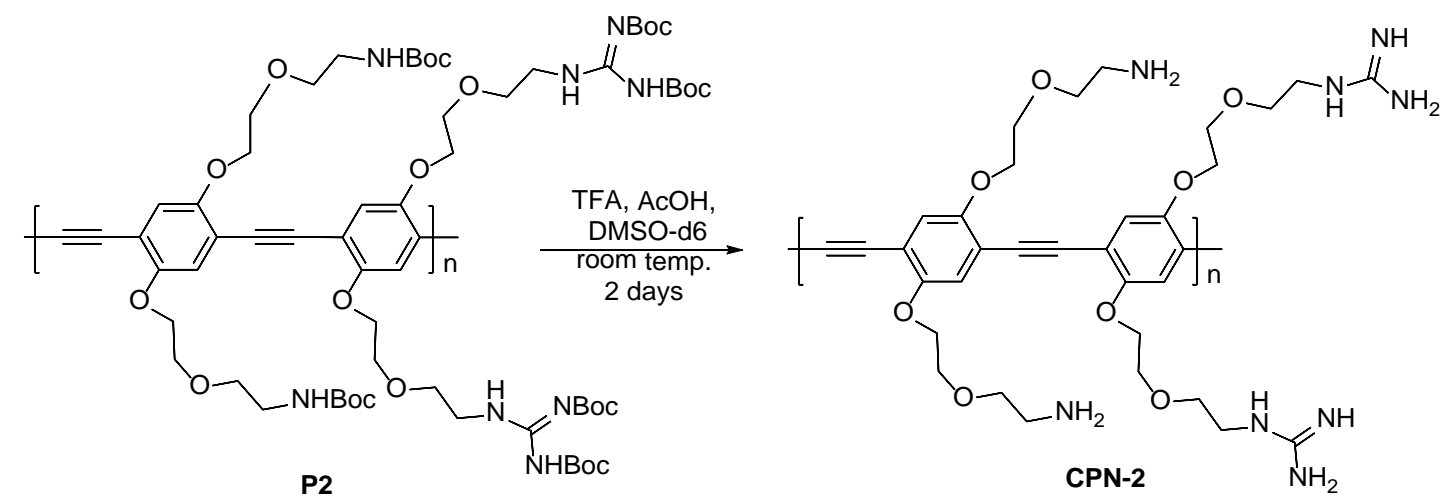

CPN-2: ${ }^{1} \mathrm{H}$ NMR (600 MHz, $\mathrm{D}_{2} \mathrm{O}$, ) ): 7.06 (br, 2H, Ar-H), 4.30-3.19 (br, 11.87H, $\mathrm{CH}_{2} \mathrm{CH}_{2} \mathrm{OCH}_{2} \mathrm{CH}_{2}$ ), 1.16 (s, 0.02H); FT-IR (Neat): v = 3310, 2863, 1560, 1419, 1359, 1301, 1273, 1199, 1110, 1053, $1020 \mathrm{~cm}^{-1}$; UVvis $\left(\mathrm{H}_{2} \mathrm{O}\right): \lambda_{\max }=411 \mathrm{~nm}$; fluo $\lambda_{\max }(400 \mathrm{~nm}$ ex $)=479 \mathrm{~nm} ; \mathrm{QY}=0.64 \%$.

P3: Detailed monomer synthesis and characterization of monomers $\mathbf{B} 2$ and $\mathbf{C}$ is described elsewhere. ${ }^{1,2}$ Using the general procedure described above, the polymerization of monomer $\mathbf{A}$ (20.0 $\mathrm{mg}, 0.0375 \mathrm{mmol}$ ), monomer B2 (22.1 mg, $0.0337 \mathrm{mmol})$, and monomer $\mathbf{C}(3.2 \mathrm{mg}, 0.00375 \mathrm{mmol})$ in the presence of $\mathrm{Pd}\left[\left(\mathrm{PPh}_{3}\right) \mathrm{Cl}_{2}\right]$ (2.6 mg, $0.00375 \mathrm{mmol}$ ) and CuI (0.4 mg, $0.00187 \mathrm{mmol}$ ) yielded P3 (17.9 mg, $0.0225 \mathrm{mmol}, 60.0 \%$ ), see scheme S3.3. CPN fabrication was carried out as described in the general procedure to yield $\mathbf{C P N}-\mathbf{3}$, see scheme S3.4. 
Scheme S3.3. Synthetic route to P3.

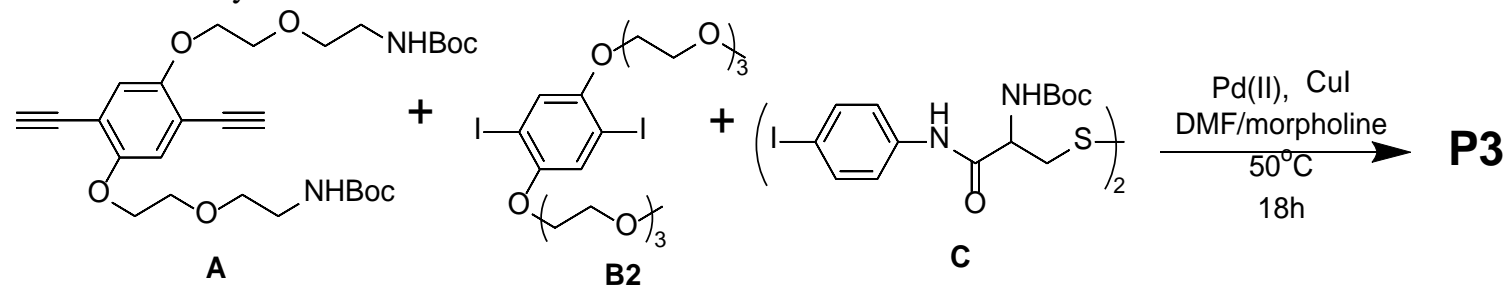

P3: ${ }^{1} \mathrm{H}$ NMR (400 MHz, DMSO-d 6 ): $\delta 8.70(\mathrm{~s}, 0.11 \mathrm{H}), 7.71(\mathrm{~s}, 0.10 \mathrm{H}), 7.51(\mathrm{~s}, 0.13 \mathrm{H}), 7.16(\mathrm{~s}, 2.10), 6.70$ (s, 1.06H), 6.39 (s, 0.12), 5.75 (s, 0.38), 4.00 (br m, 4.00H), 3.80 (br m, 4.87), 3.66 (br m, 2.27H), 3.19 (br m, 3.44H), 1.35 (s, 11.18 H). FT-IR (neat): 3002, 2778, 2505, 1654, 1486, 1462, 1429, 1362, 1321, 1244, 1176, 1133, 1052, $1043 \mathrm{~cm}^{-1}$. GPC: $\mathrm{M}_{\mathrm{w}}=23,181 \mathrm{Da}, \mathrm{M}_{\mathrm{n}}=14,489 \mathrm{Da}, \mathrm{PDI}=1.59 . \mathrm{UV}-\mathrm{Vis}$ (DMF) $\lambda_{\max }=$ $439 \mathrm{~nm}$, fluo $\lambda_{\max }(400 \mathrm{~nm}$ ex $)=511 \mathrm{~nm}, \mathrm{QY}=39 \%$.

Scheme S3.4. Boc-deprotection of P3 to yield CPN-3.
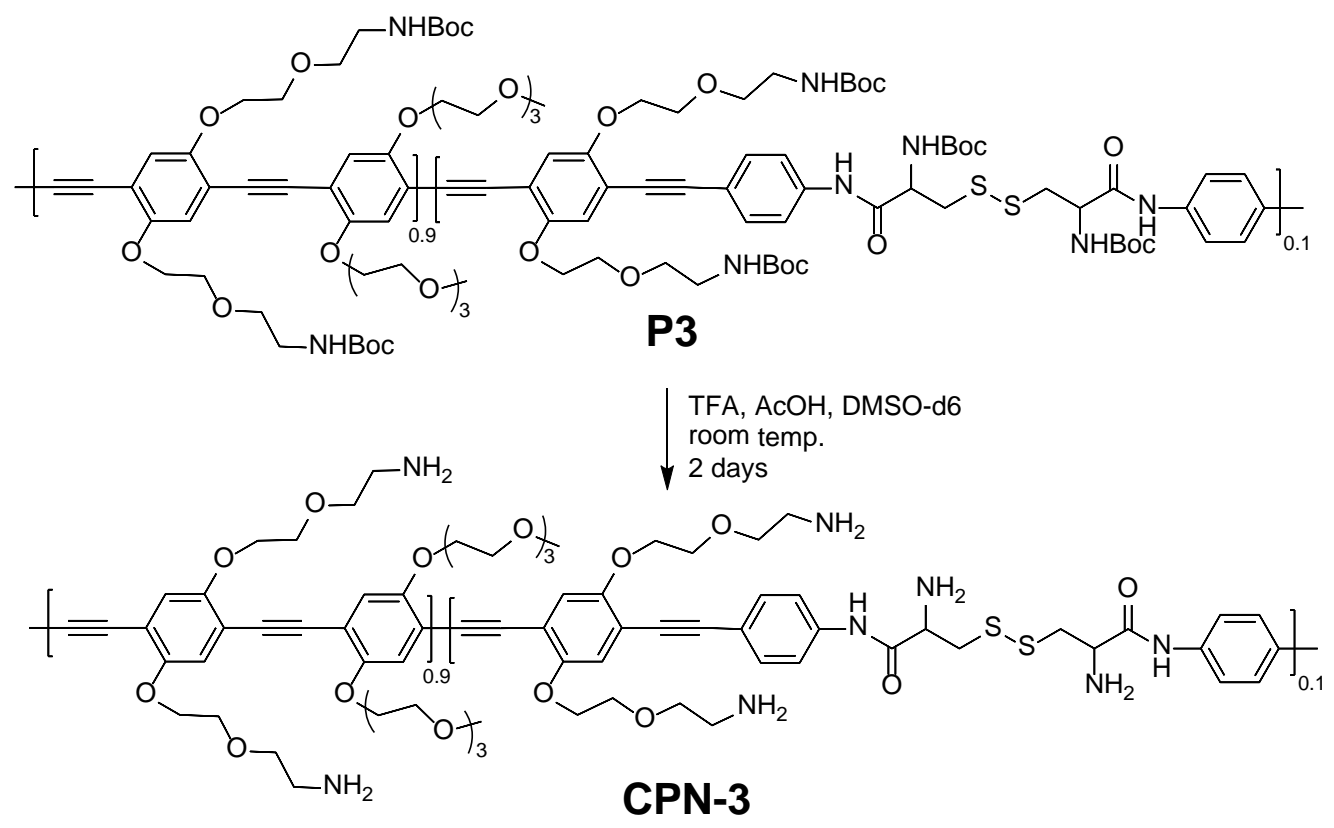

CPN-3: ${ }^{1} \mathrm{H}$ NMR (600 MHz, $\mathrm{D}_{2} \mathrm{O}, \delta$ ): 7.00 (br, 2H, Ar-H), 4.24-3.26 (br, $17.34 \mathrm{H}, \mathrm{CH}_{2} \mathrm{CH}_{2} \mathrm{OCH}_{2} \mathrm{CH}_{2}$ ); FTIR (Neat): $v=3357,2873,1581,1444,1420,1351,1302,1199,1093,1047 \mathrm{~cm}^{-1}$; UV-vis $\left(\mathrm{H}_{2} \mathrm{O}\right): \lambda_{\max }=422$ $\mathrm{nm}$; fluo $\lambda_{\max }(400 \mathrm{~nm}$ ex $)=464 \mathrm{~nm} ; \mathrm{QY}=0.36 \%$.

P4: Detailed monomer and polymer synthesis, CPN fabrication and characterization is described elsewhere. ${ }^{23,28}$ 


\subsubsection{CPN/GAG complexation.}

Sodium hyaluronate (HA) was purchased from Lifecore (MW $100 \mathrm{~K}$ ) and used as received. A stock solution was prepared by dissolving $2.0 \mathrm{mg}$ of $\mathrm{HA}$ in $1 \mathrm{~mL}$ of deionized water. Heparin sodium was purchased from Acros Organics and used as received. A stock solution was prepared by dissolving $4.0 \mathrm{mg}$ of $\mathrm{HS}$ in $1 \mathrm{~mL}$ of deionized water. Chondroitin sulfate A (CS) and chondroitin sulfate B (dermatan sulfate, DS) were purchased from Sigma Aldrich and used as received. Stock solutions of CS and DS were prepared by dissolving $2.0 \mathrm{mg}$ and $1.0 \mathrm{mg}$, respectively, in $1 \mathrm{~mL}$ of deionized water. CPN and GAG were mixed and allowed to incubate for 30 minutes prior to measurements. For consistency between the CPNs, the concentration was adjusted to give an optical density of 0.1 , which corresponds to approximately $10 \mu \mathrm{M}$ (based on polymer repeating unit). The GAG concentration used was $30 \mu \mathrm{M}$ (based on GAG repeating unit). Samples were prepared in deionized water, unless otherwise stated.

\subsubsection{Determination of hydrodynamic diameters of CPNs.}

Light scattering measurements were performed with a LM10 HS (NanoSight, Amesbury, United Kingdom), equipped with a sCMOS camera, sample chamber with a $488 \mathrm{~nm}$ blue laser, and Viton fluoroelastomer oring. The samples were prepared in similar manner for absorption and emission measurements using water $(18 \Omega)$ filtered through $0.45 \mu \mathrm{m}$ PTFE syringe filters. The samples were injected into the sample chamber with $1 \mathrm{~mL}$ sterile syringes (Restek Corporation, Pennsylvania, USA) until the liquid reached the tip of the nozzle. All measurements were performed at $25^{\circ} \mathrm{C}$ using a LM14C temperature controller (NanoSight, Amesbury, United Kingdom). Each sample was measured three times.

\subsubsection{Determination of zeta potentials of CPNs.}

Dynamic light scattering measurements were performed by Zetasizer nano-ZS (Zen 3600, Malvern Instruments Ltd.) using a folded capillary cell (Catalog \# DTS1060), at room temperature. The samples were prepared at approximately $0.5 \mathrm{mM}$ in water $(18 \Omega)$, which was filtered through $0.45 \mu \mathrm{M}$ PTFE syringe filter. Each sample was measured six times. 


\subsubsection{Hierarchical cluster analysis (HCA) of emission spectra.}

The commercially available statistical software JMP ${ }^{\circledR}$ (version 11) was used for analysis. For simplicity, one CPN emission data set in water (triplicate samples) was used for HCA to demonstrate role of functional group in differentiation. The default distance calculation method, Ward's, was used for cluster distances.

\subsubsection{CPN in synthetic urine.}

Surine $^{\mathrm{TM}}$ was purchased from Dyna-Tek Industries, Inc. (product \#720) and used as received. Surine ${ }^{\mathrm{TM}}$ is a urine simulant, with a proprietary formula, which contains creatinine and urea, and was used as media for CPN/GAG measurements. For the differentiation application and to simulate detection of GAGs present in urine, GAGs $(100 \mathrm{nM})$ were mixed in Surine ${ }^{\mathrm{TM}}$, then CPN was added to the GAG-containing urine simulant and allowed to incubate for 30 minutes prior to measurements.

\subsubsection{LDA of emission spectra.}

The commercially available statistical software $\mathrm{JMP}^{\circledR}$ (version 11) was used for analysis. The data included in the training matrix were the emission spectra ratios of CPN to CPN+GAG in urine simulant. The matrix training set was 4 CPNs $x 4$ GAGs $x 3$ replicates, an input of 48 data sets (spectrum ratios). The discrimination method used was the linear, common covariance method.

\subsubsection{Supporting data}

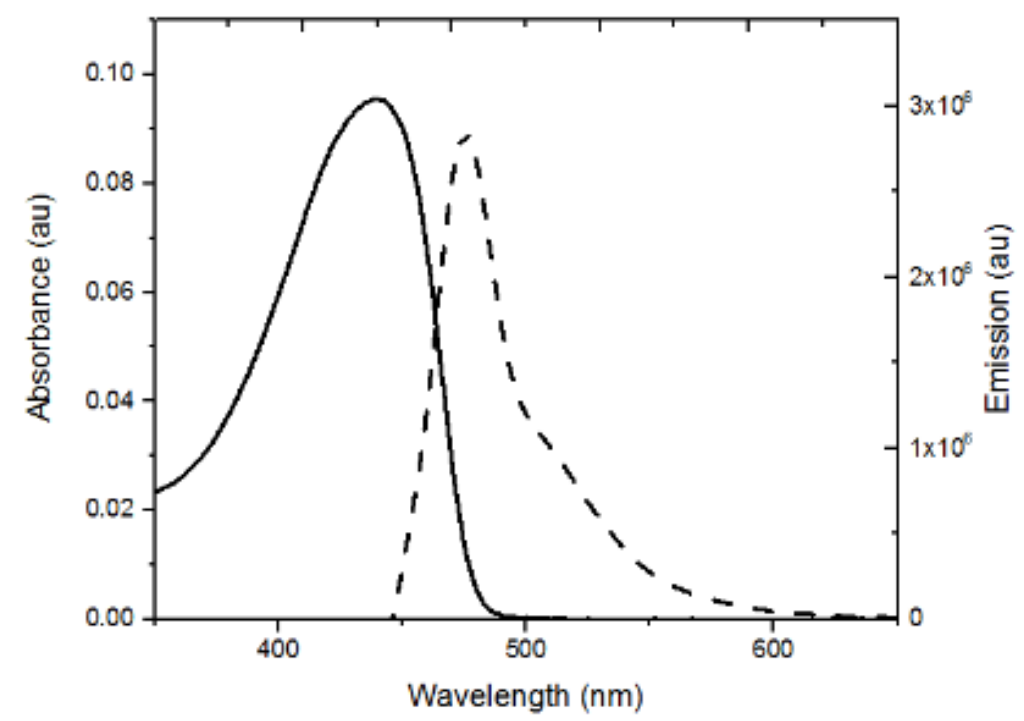

Fig. S3.1 Absorption and emission spectra for P2 in DMF (Excitation $=430 \mathrm{~nm}$, slit widths $=3 \mathrm{~nm}$, integration time $=0.1 \mathrm{~s}$ ). 


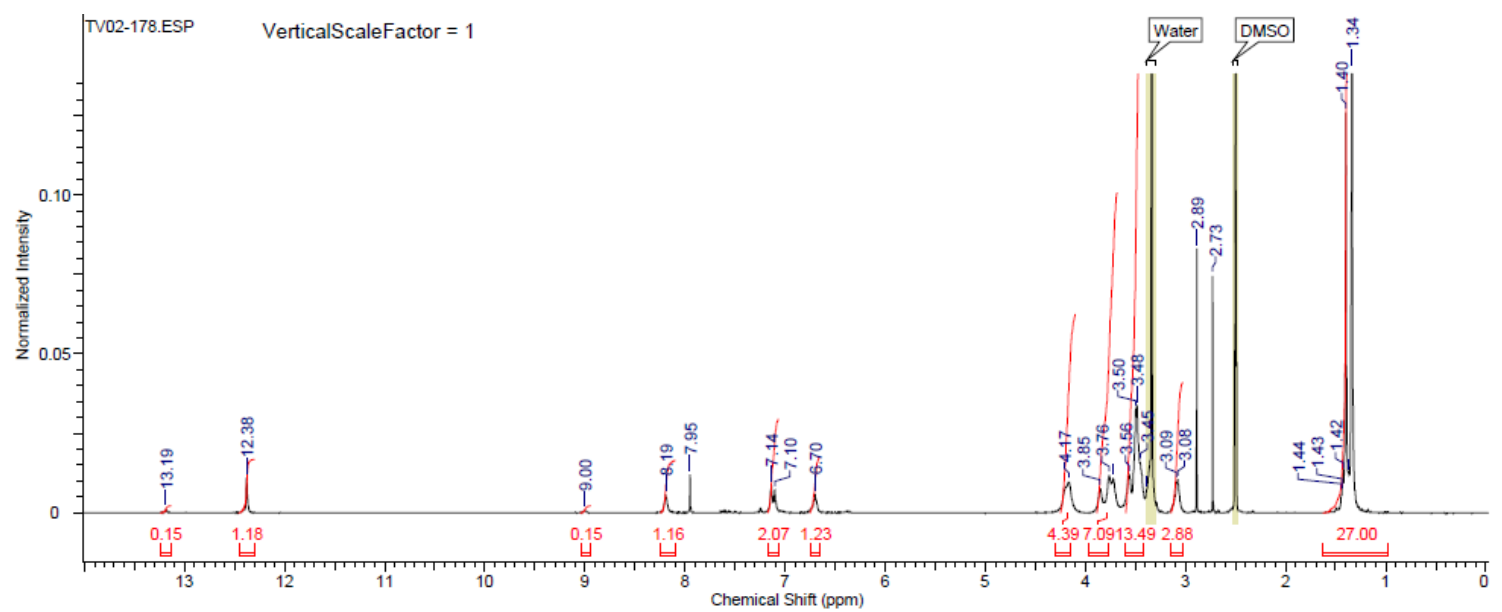

Fig. S3.2 ${ }^{1} \mathrm{H}$ NMR (400 MHz) of P2 in DMSO-d6.

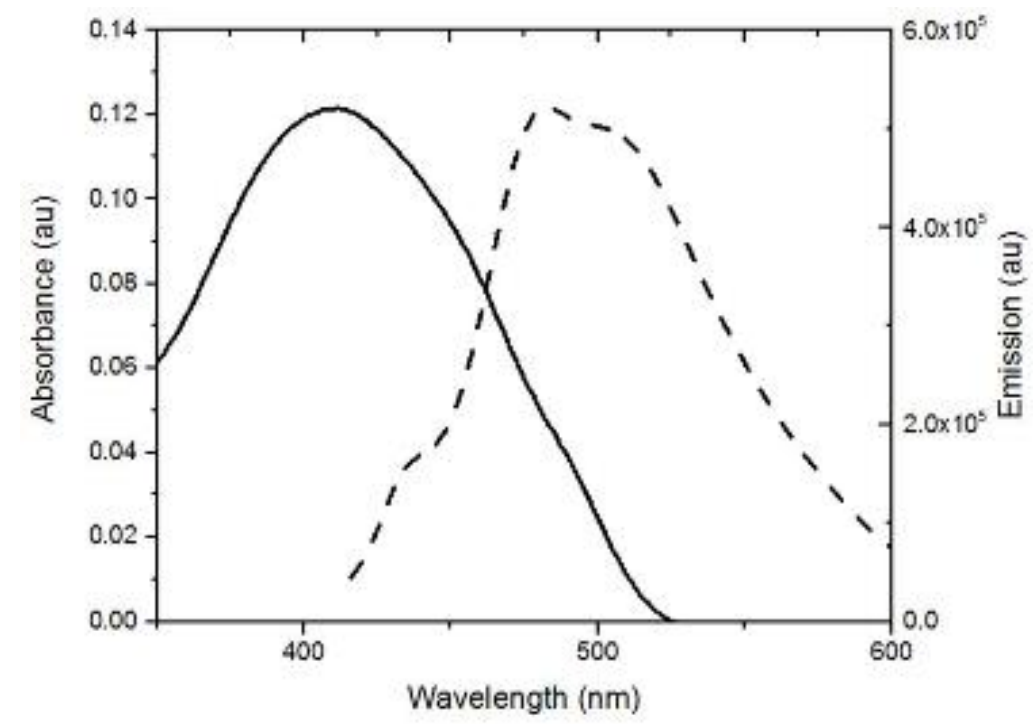

Fig. S3.3 Absorption and emission spectra for CPN-2 in water (Excitation $=400 \mathrm{~nm}$, slit widths $=3 \mathrm{~nm}$, integration time $=0.1 \mathrm{~s}$ ).

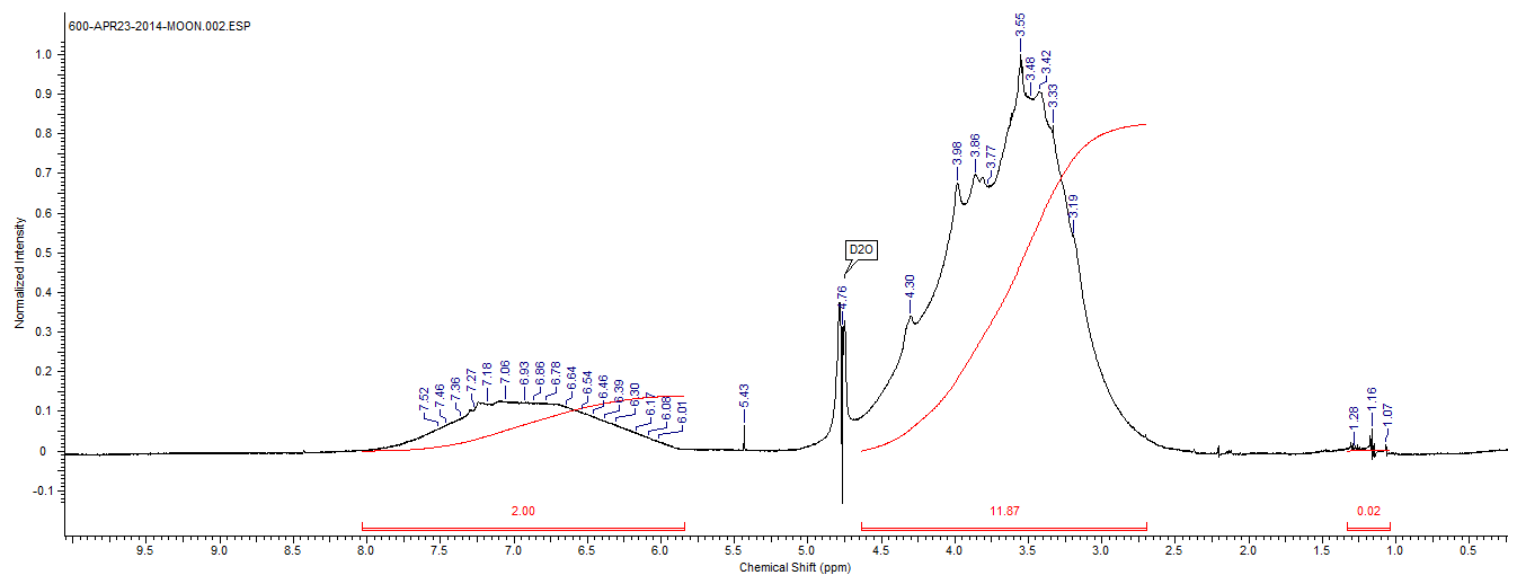

Fig. S3.4 ${ }^{1} \mathrm{H}$ NMR (600 MHz) of $\mathbf{C P N}-2$ in $\mathrm{D}_{2} \mathrm{O}$. 


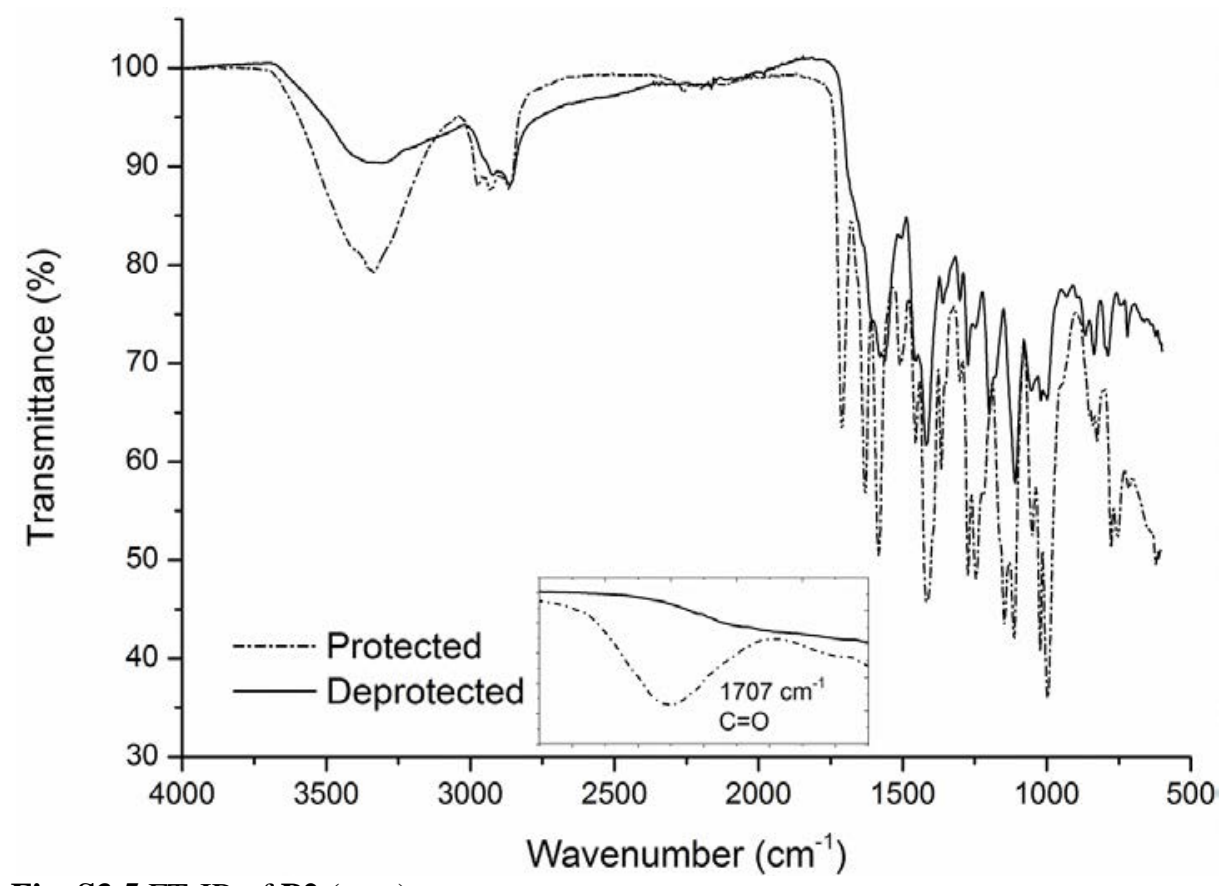

Fig. S3.5 FT-IR of P2 (neat).

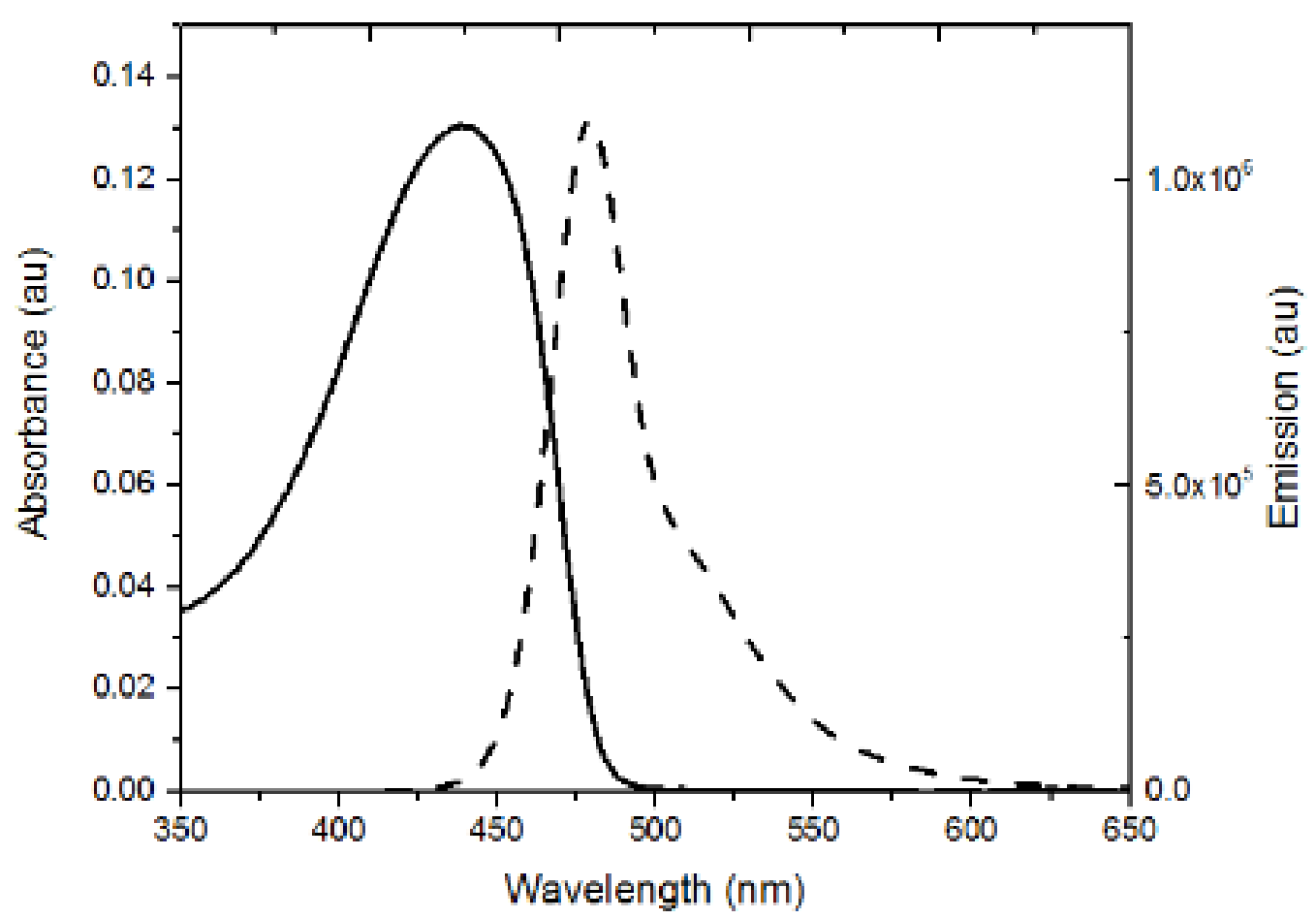

Fig. S3.6 Absorption and emission spectra for P3 in DMF (Excitation $=400 \mathrm{~nm}$, slit widths $=3 \mathrm{~nm}$, integration time $=0.1 \mathrm{~s}$ ). 


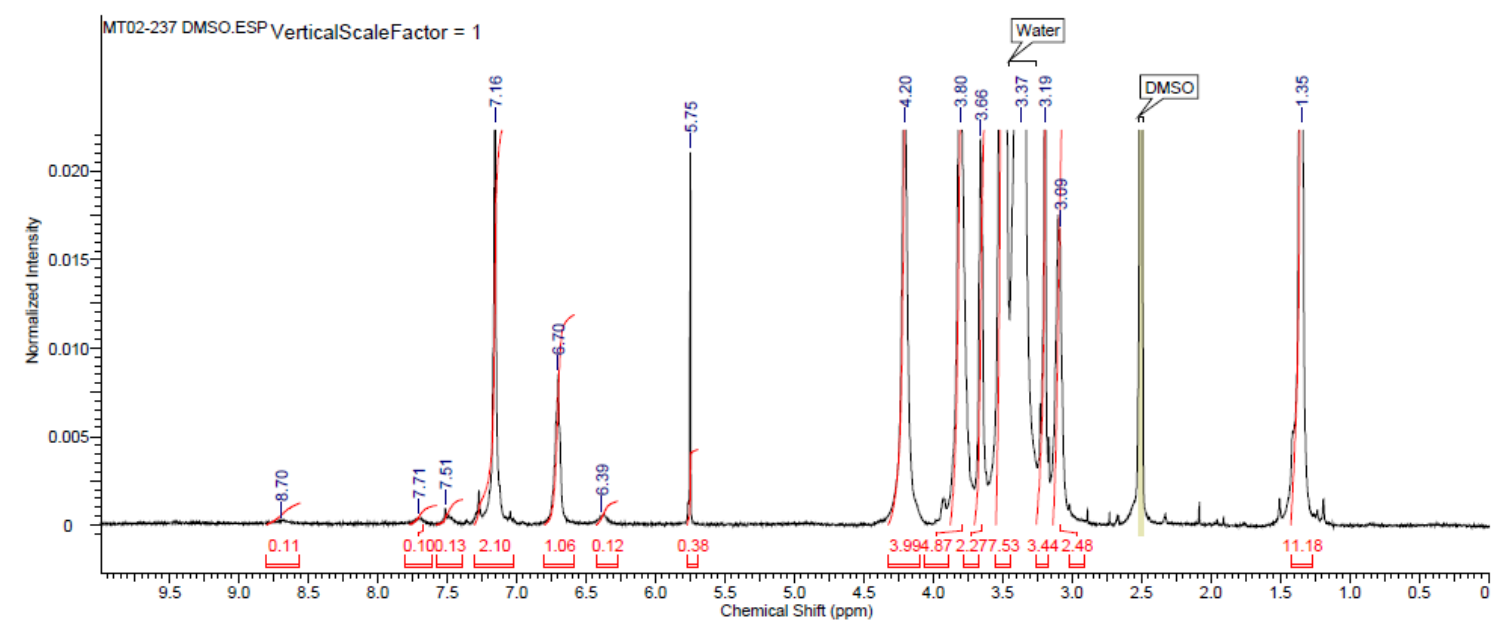

Fig. S3.7 ${ }^{1} \mathrm{H}$ NMR (400 MHz) of P3 in DMSO-d6.

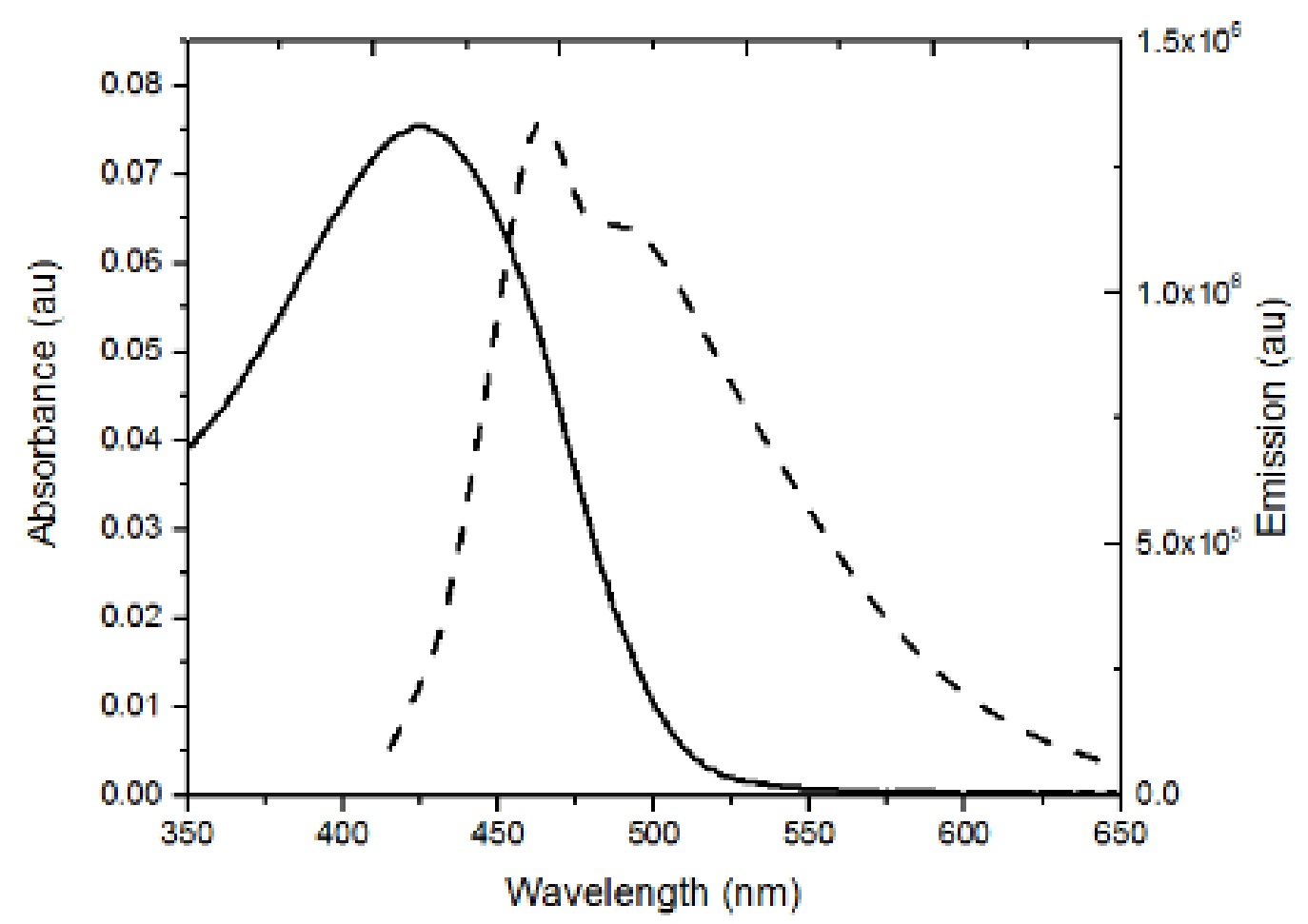

Fig. S3.8 Absorption and emission spectra for CPN-3 in water (Excitation $=400 \mathrm{~nm}$, slit widths $=3 \mathrm{~nm}$, integration time $=0.1 \mathrm{~s}$ ). 


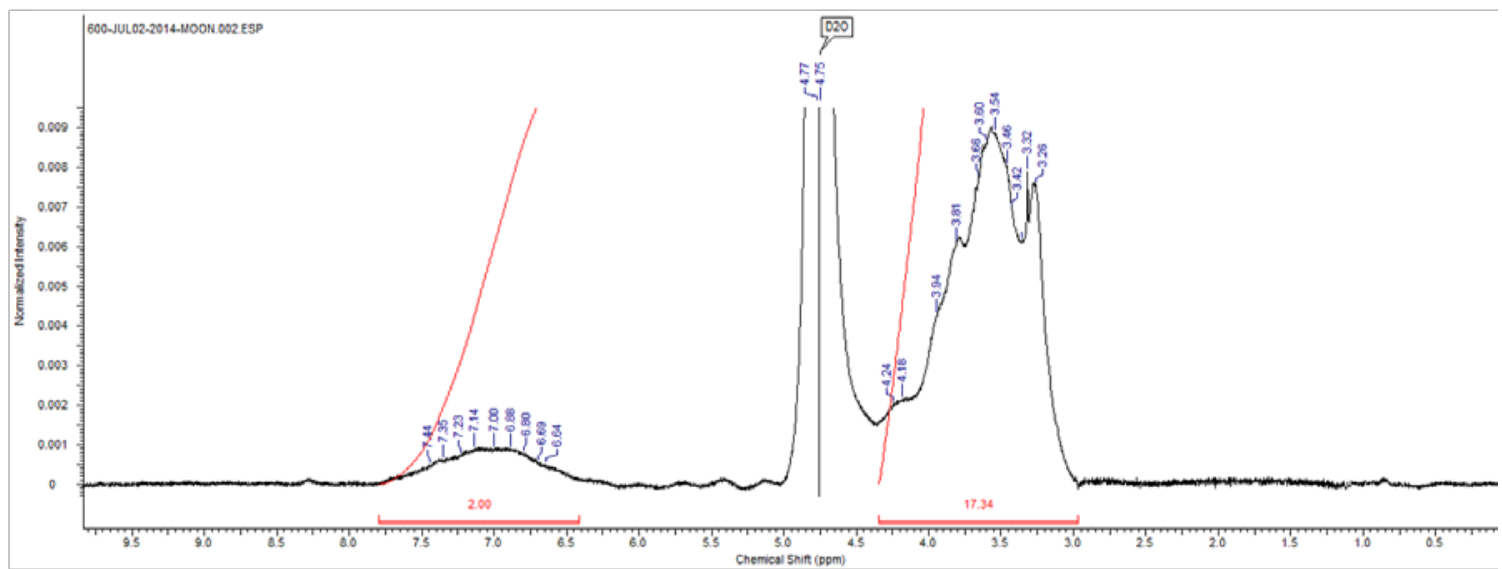

Fig. S3.9 ${ }^{1} \mathrm{H}$ NMR (600 MHz) of $\mathbf{C P N}-3$ in $\mathrm{D}_{2} \mathrm{O}$.

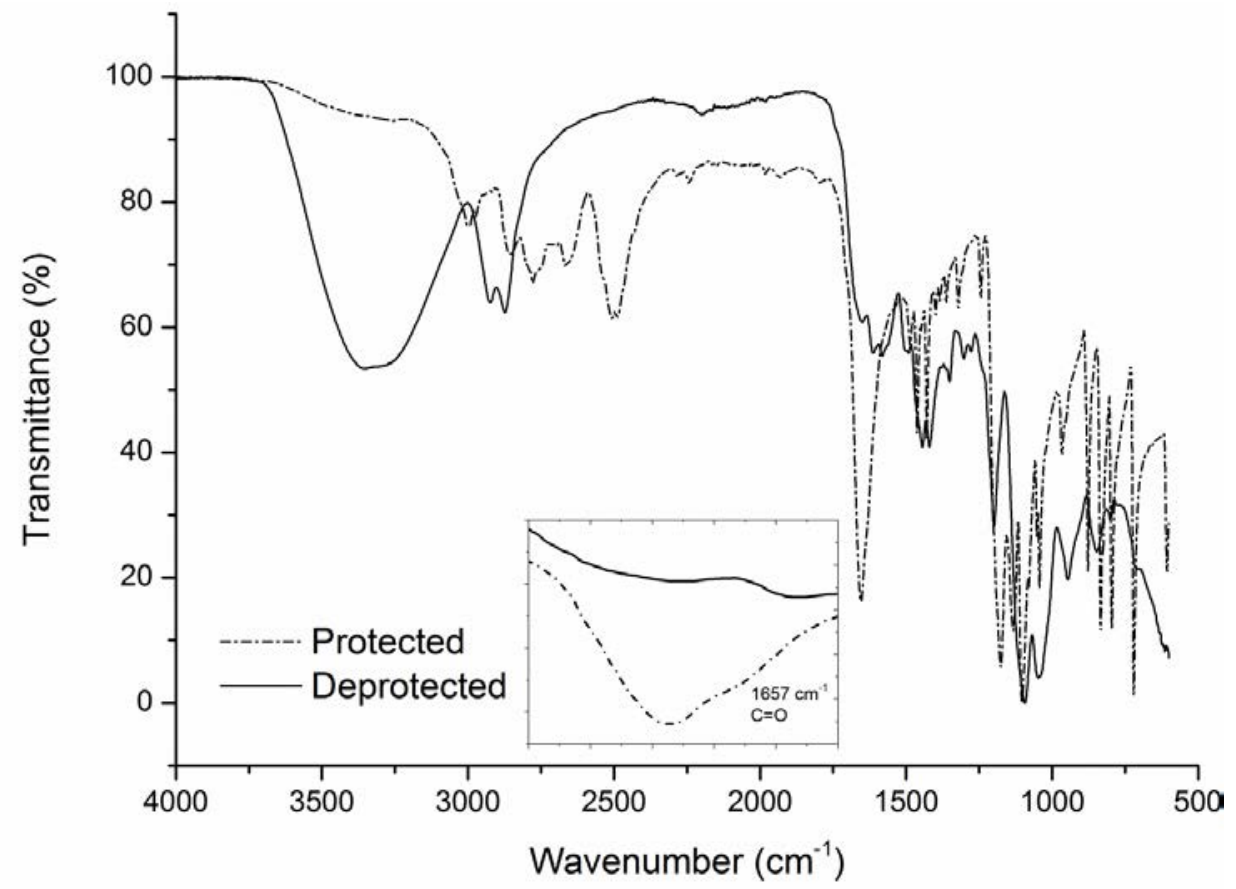

Fig. S3.10 FT-IR of P3 (neat). 


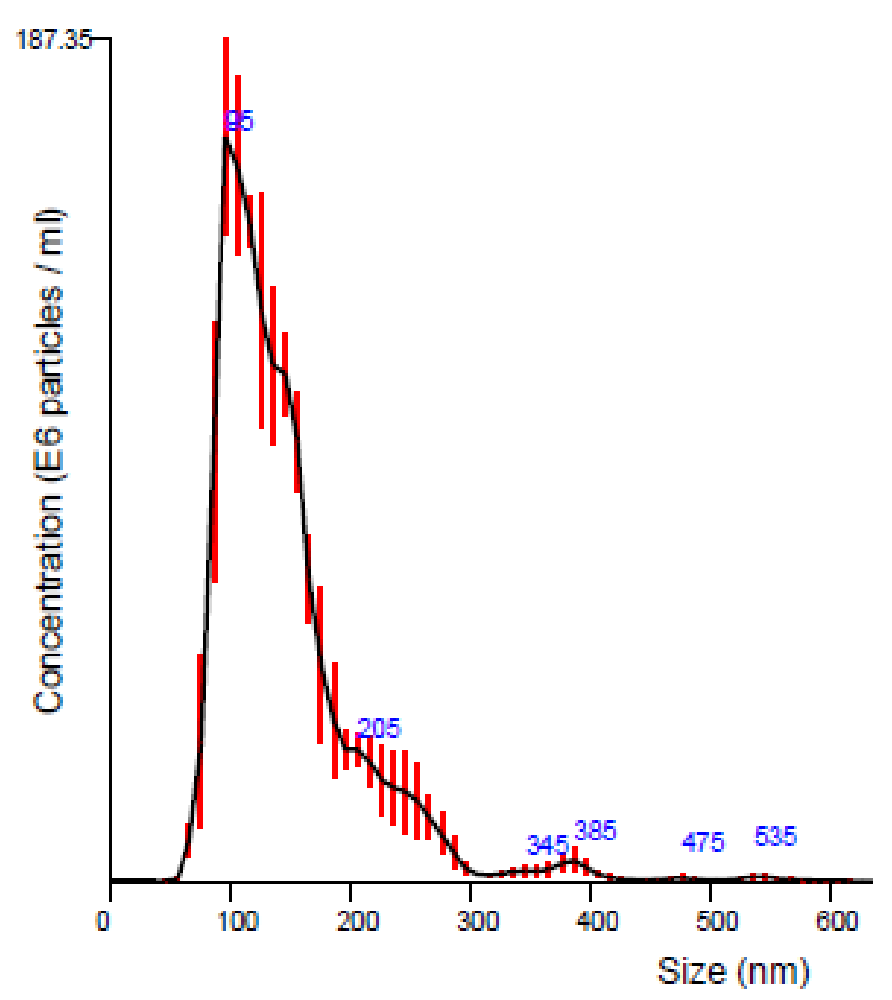

Fig. S3.11 NTA of CPN-1 in water.

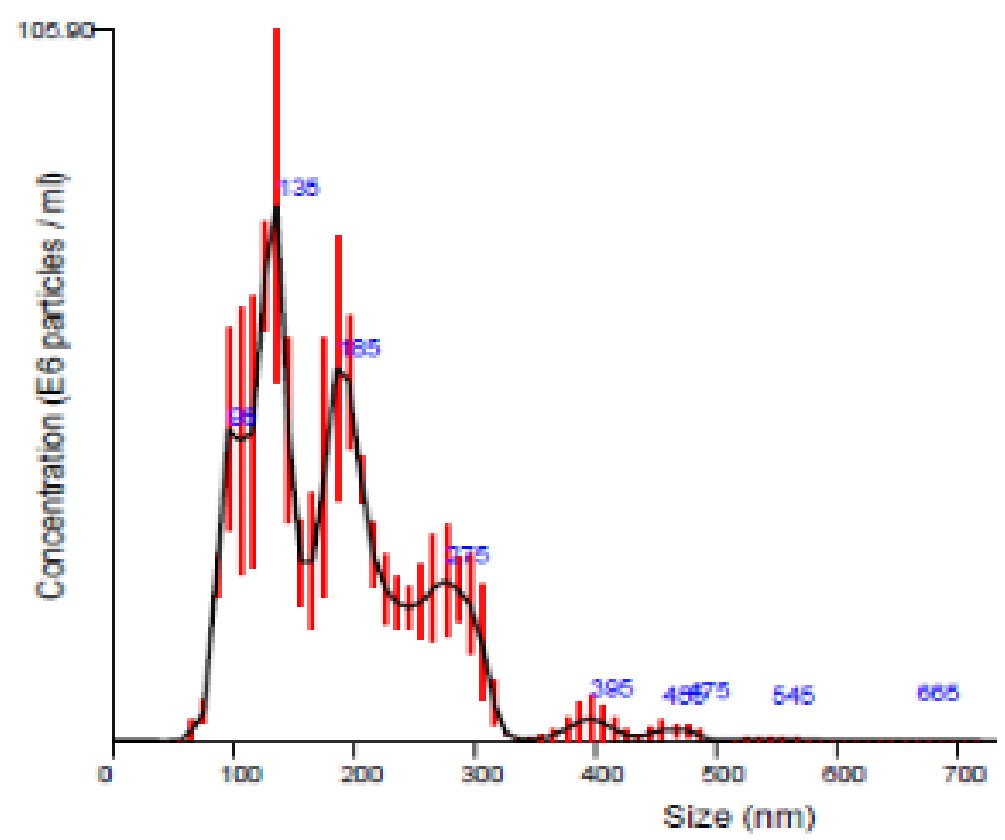

Fig. S3.12 NTA of CPN-2 in water. 


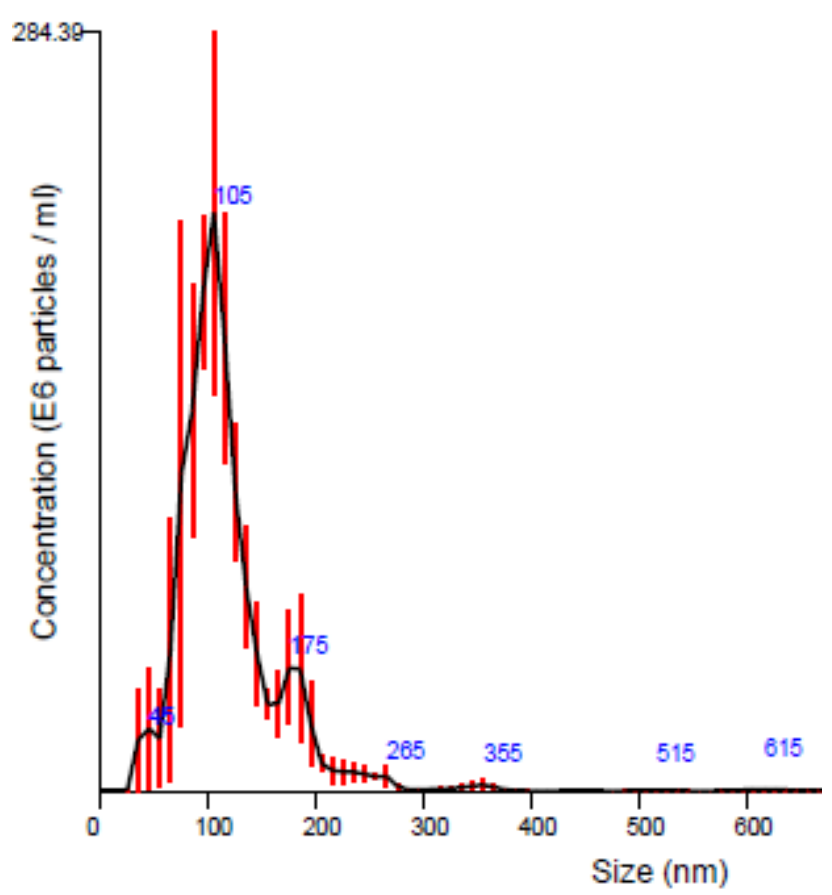

Fig. S3.13 NTA of CPN-3 in water.

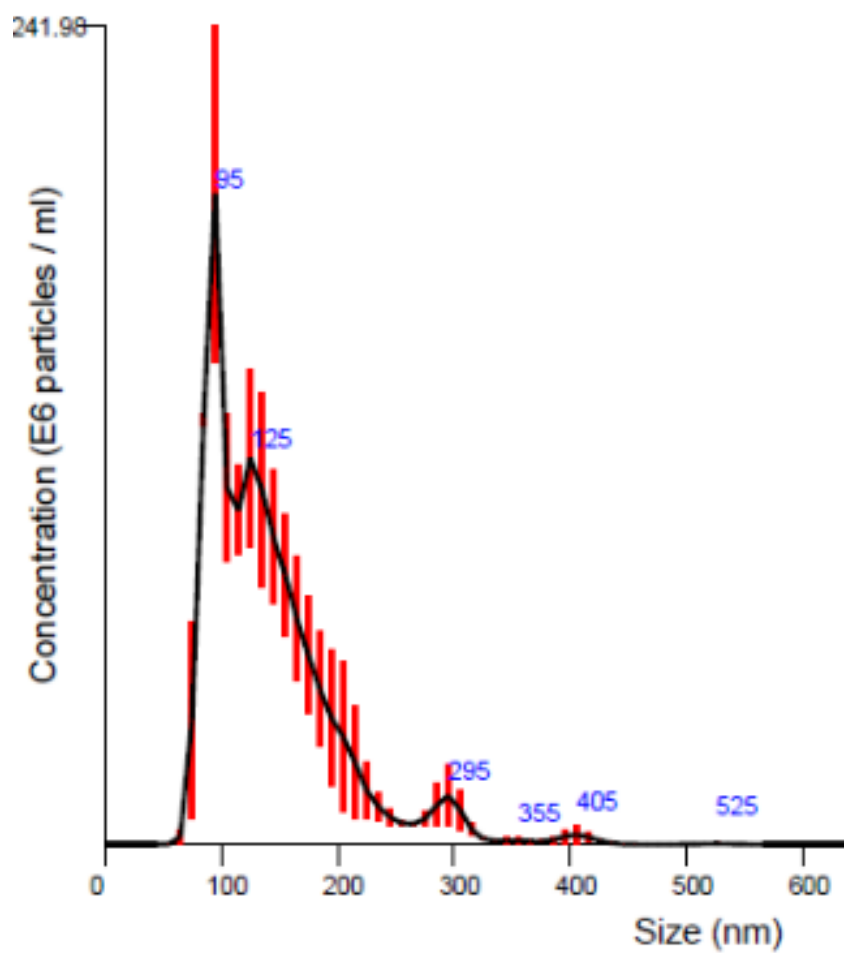

Fig. S3.14 NTA of CPN-4 in water. 


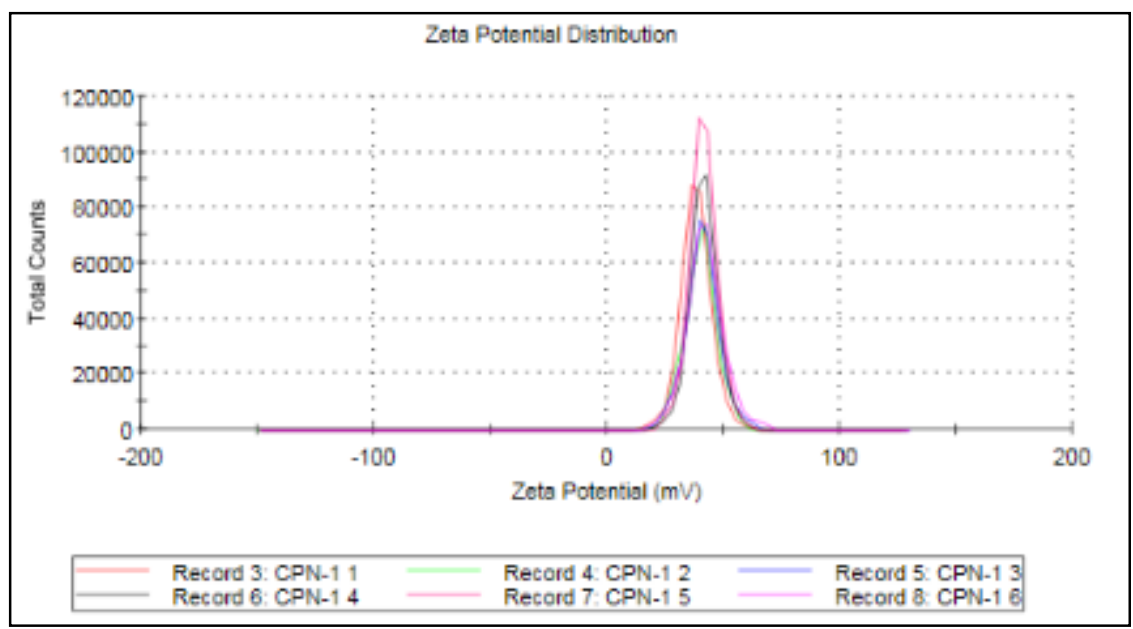

Fig. S3.15 Zeta potential of CPN-1 in water.

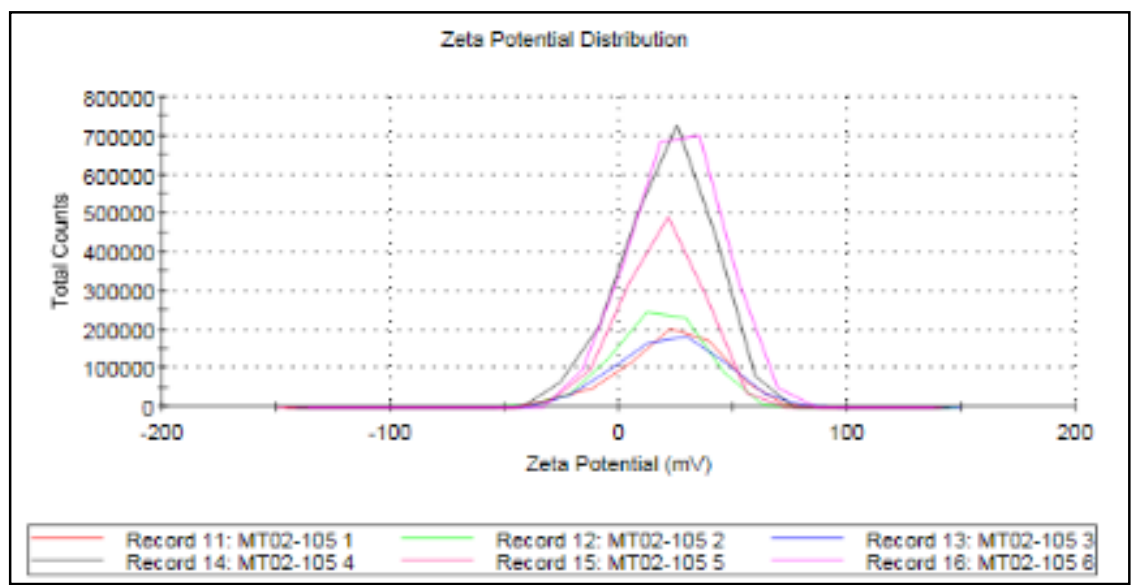

Fig. S3.16 Zeta potential of CPN-2 in water.

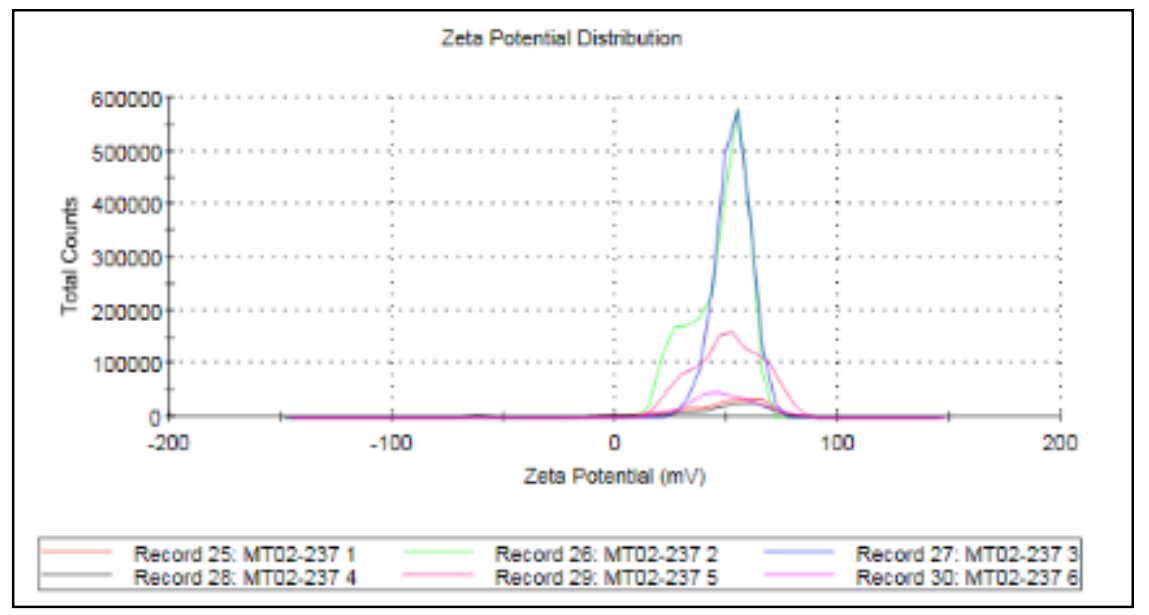

Fig. S3.17 Zeta potential of CPN-3 in water. 


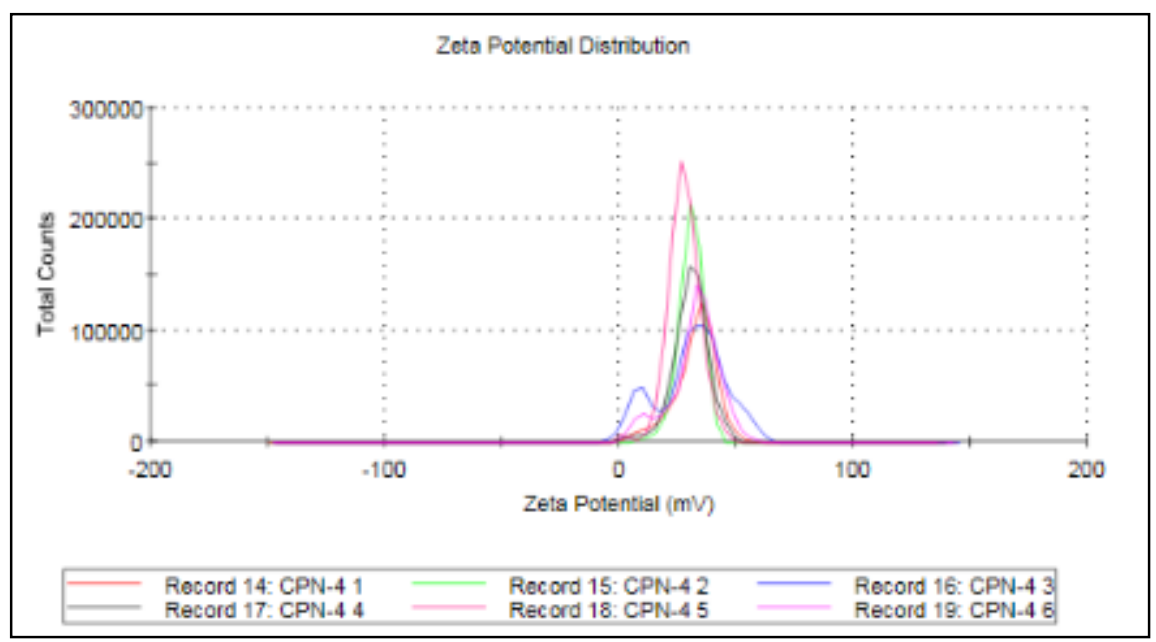

Fig. S3.18 Zeta potential of CPN-4 in water.

Table S3.1 Summary of NTA data for CPN/GAG complexes.

\begin{tabular}{|c|c|c|c|}
\hline Sample & $\begin{array}{c}\text { Mean } \\
(\mathrm{nm})\end{array}$ & $\begin{array}{c}\text { Mode } \\
(\mathrm{nm})\end{array}$ & $\begin{array}{l}\mathrm{SD}^{\mathrm{a}} \\
(\mathrm{nm})\end{array}$ \\
\hline CPN-1 & $140 \pm 0.9$ & $108 \pm 8.4$ & $63 \pm 3.2$ \\
\hline$+\mathrm{HA}$ & $174 \pm 1.4$ & $145 \pm 9.4$ & $65 \pm 3.9$ \\
\hline$+\mathrm{HS}$ & $171 \pm 1.9$ & $142 \pm 7.7$ & $58 \pm 1.9$ \\
\hline$+\mathrm{CS}$ & $177 \pm 4.8$ & $148 \pm 13.3$ & $82 \pm 8.2$ \\
\hline$+\mathrm{DS}$ & $167 \pm 1.6$ & $159 \pm 0.6$ & $58 \pm 3.2$ \\
\hline CPN-2 & $179 \pm 6.3$ & $125 \pm 6.5$ & $74 \pm 5.8$ \\
\hline$+\mathrm{HA}$ & $152 \pm 2.7$ & $138 \pm 8.5$ & $62 \pm 5.3$ \\
\hline$+\mathrm{HS}$ & $162 \pm 1.1$ & $124 \pm 7.1$ & $70 \pm 3.3$ \\
\hline$+\mathrm{CS}$ & $137 \pm 2.1$ & $111 \pm 6.0$ & $59 \pm 3.7$ \\
\hline$+\mathrm{DS}$ & $137 \pm 2.3$ & $114 \pm 3.2$ & $59 \pm 3.7$ \\
\hline CPN-3 & $118 \pm 10.1$ & $90 \pm 7.8$ & $66 \pm 23.5$ \\
\hline$+\mathrm{HA}$ & $150 \pm 4.6$ & $125 \pm 4.6$ & $57 \pm 4.7$ \\
\hline$+\mathrm{HS}$ & $151 \pm 5.4$ & $125 \pm 2.5$ & $45 \pm 4.9$ \\
\hline$+\mathrm{CS}$ & $165 \pm 6.4$ & $149 \pm 1.6$ & $60 \pm 5.1$ \\
\hline$+\mathrm{DS}$ & $140 \pm 1.3$ & $125 \pm 4.2$ & $47 \pm 3.5$ \\
\hline $\mathrm{CPN}-4$ & $135 \pm 8.1$ & $95 \pm 8.1$ & $55 \pm 3.8$ \\
\hline$+\mathrm{HA}$ & $170 \pm 4.4$ & $132 \pm 6.6$ & $75 \pm 8.2$ \\
\hline$+\mathrm{HS}$ & $187 \pm 2.7$ & $144 \pm 5.3$ & $87 \pm 11.8$ \\
\hline$+\mathrm{CS}$ & $216 \pm 2.6$ & $151 \pm 3.7$ & $97 \pm 5.2$ \\
\hline$+\mathrm{DS}$ & $216 \pm 1.9$ & $148 \pm 6.6$ & $92 \pm 2.8$ \\
\hline
\end{tabular}

${ }^{a} \mathrm{SD}$ is the standard deviation characteristic of the width of the mean peak. 
Table S3.2 Summary of canonical scores from LDA plot.

\begin{tabular}{|c|c|c|c|c|c|}
\hline Canonical & Eigenvalue & $\begin{array}{c}\text { Percent of } \\
\text { variation }\end{array}$ & $\begin{array}{c}\text { Canonical } \\
\text { correlation }\end{array}$ & $\begin{array}{c}\text { Likelihood } \\
\text { ratio }\end{array}$ & P-value \\
\hline 1 & 51.1592 & 71.0561 & 0.9903 & 0.00015 & $<0.0001$ \\
\hline 2 & 12.0357 & 16.7166 & 0.9608 & 0.00782 & $<0.0001$ \\
\hline 3 & 8.8034 & 12.2273 & 0.9476 & 0.10200 & $<0.0001$ \\
\hline
\end{tabular}

Table S3.3 Squared Mahalanobis distances to each group centroid.

\begin{tabular}{|c|c|c|c|c|c|}
\hline Row & Actual & CS & DS & HA & HS \\
\hline 1 & CS & 40.333331755 & 12294.647468 & 4063.5247309 & 7101.4731662 \\
\hline 2 & CS & 40.33333189 & 12318.702339 & 4091.8694316 & 7100.0157848 \\
\hline 3 & CS & 40.333331247 & 12295.354372 & 4055.8629135 & 7077.7114241 \\
\hline 4 & CS & 40.333332814 & 12320.127561 & 4063.8690325 & 7028.8745497 \\
\hline 5 & CS & 40.333332332 & 12284.219683 & 4022.5165612 & 7068.8817155 \\
\hline 6 & $\mathrm{CS}$ & 40.33333311 & 12305.608646 & 4078.0823462 & 7070.0813947 \\
\hline 7 & CS & 40.333332299 & 12324.225381 & 4101.1928134 & 7029.6991414 \\
\hline 8 & $\mathrm{CS}$ & 40.333332583 & 12297.892499 & 4043.5769265 & 7080.2601624 \\
\hline 9 & CS & 40.333332368 & 12290.603231 & 4079.2351324 & 7099.9786147 \\
\hline 10 & CS & 40.333332042 & 12317.900963 & 4084.3114748 & 7104.2194088 \\
\hline 11 & CS & 40.333332023 & 12321.138359 & 4086.9472166 & 7088.7210233 \\
\hline 12 & CS & 40.333331329 & 12327.718608 & 4068.9691099 & 7091.0784818 \\
\hline 13 & DS & 12336.168794 & 40.333332198 & 4485.4027952 & 5017.6750565 \\
\hline 14 & DS & 12308.32746 & 40.333331405 & 4461.5478914 & 5024.6443335 \\
\hline 15 & DS & 12285.66102 & 40.333332437 & 4444.505515 & 4960.6845733 \\
\hline 16 & DS & 12322.927181 & 40.333333024 & 4494.3974088 & 5092.3506657 \\
\hline 17 & DS & 12284.738557 & 40.33333405 & 4431.2023819 & 5018.2556851 \\
\hline 18 & DS & 12309.48622 & 40.333333605 & 4465.5395729 & 4968.2806311 \\
\hline 19 & DS & 12308.350358 & 40.333333771 & 4497.3940618 & 4981.3115882 \\
\hline 20 & DS & 12299.854928 & 40.333333821 & 4458.0858604 & 5006.5912977 \\
\hline 21 & DS & 12288.977123 & 40.333333818 & 4449.3440504 & 5029.0243225 \\
\hline 22 & DS & 12316.660014 & 40.333333525 & 4479.2810227 & 5006.1744725 \\
\hline 23 & DS & 12309.957045 & 40.333332342 & 4471.8327096 & 5016.2428069 \\
\hline 24 & DS & 12327.030404 & 40.333332195 & 4490.8880232 & 5031.904691 \\
\hline 25 & HA & 4071.5727552 & 4479.4540603 & 40.333334415 & 4189.6090669 \\
\hline 26 & HA & 4051.7432194 & 4464.1781327 & 40.333333094 & 4181.7118782 \\
\hline 27 & HA & 4038.7851613 & 4447.7852168 & 40.333333772 & 4226.4907325 \\
\hline 28 & HA & 4069.2033941 & 4452.9086196 & 40.333333224 & 4166.0945872 \\
\hline 29 & HA & 4043.7940084 & 4465.4116832 & 40.33333387 & 4101.2929977 \\
\hline 30 & HA & 4091.1485334 & 4485.9916447 & 40.333333783 & 4207.6709894 \\
\hline 31 & HA & 4087.8395223 & 4435.7896652 & 40.333333211 & 4216.5914613 \\
\hline 32 & HA & 4079.9438813 & 4435.5311514 & 40.333333177 & 4219.9600424 \\
\hline 33 & HA & 4067.9651299 & 4520.1123035 & 40.333333286 & 4148.2627038 \\
\hline
\end{tabular}




\begin{tabular}{|l|c|c|c|c|c|}
\hline 34 & HA & 4091.700492 & 4486.0424938 & 40.333333274 & 4184.9552956 \\
\hline 35 & HA & 4093.2678457 & 4481.8711676 & 40.333333602 & 4202.0467871 \\
\hline 36 & HA & 4052.9937363 & 4474.345146 & 40.333333464 & 4153.3574859 \\
\hline 37 & HS & 7096.5304652 & 5022.950778 & 4191.1591296 & 40.333333658 \\
\hline 38 & HS & 7074.0622865 & 5010.9473948 & 4206.4649295 & 40.333333755 \\
\hline 39 & HS & 7059.2479562 & 4991.6573071 & 4181.3105117 & 40.333334356 \\
\hline 40 & HS & 7096.8954728 & 5039.1040348 & 4194.8850944 & 40.333334461 \\
\hline 41 & HS & 7070.6823557 & 4973.1371849 & 4165.069548 & 40.33333396 \\
\hline 42 & HS & 7087.2351087 & 5032.825679 & 4206.7409419 & 40.333333788 \\
\hline 43 & HS & 7056.5975622 & 4986.0591011 & 4134.249703 & 40.33333355 \\
\hline 44 & HS & 7081.3330154 & 5052.2305167 & 4202.4199089 & 40.3333335 \\
\hline 45 & HS & 7060.9924572 & 4989.4852004 & 4150.13598 & 40.333333518 \\
\hline 46 & HS & 7083.6742369 & 5025.4448787 & 4194.4434217 & 40.333333149 \\
\hline 47 & HS & 7095.8214509 & 5018.3121938 & 4188.5907513 & 40.333333749 \\
\hline 48 & HS & 7077.9224838 & 5010.9858432 & 4182.5741073 & 40.333332594 \\
\hline
\end{tabular}

3.7 References

1. V. Lokeshwar, M. Selzer, D. Unwala, V. Estrella, M. Gomez, R. R. Golshani, R. Kester, D. Klumpp, A. Gousse. J.Urol. 2006, 176, 1001-1007.

2. E. Nabih, M. Sayed. Egyptian Journal of Chest Diseases and Tuberculosis. 2013, 62, 343-348.

3. R. Soler, H. Brushini, J. Truzzi, J. Martins, N. Camara, M. Alves, K. Leit, H. Nader, M. Srougi, V. Ortiz. Int Braz J Urol. 2008, 34, 503-511.

4. G. Yip, M. Smollich, M. Gotte. Mol Cancer Ther. 2008, 5, 2139-2148.

5. M. Kalita, S. Balivada, V. P. Swarup, C. Mencio, K. Raman, U. R. Desai, D. Troyer, B. Kuberan. J. Am. Chem. Soc. 2014, 136, 554-7.

6. N. Volpi, F. Maccari, R. Linhardt. J.Anal. Biochem. 2009, 388, 140-145.

7. D. J. Langeslay, C. N.Beecher, A. Naggi, M. Guerrini, G.Torri, C. K. Larive. Anal. Chem. 2013, $85,1247-1255$.

8. J. Spencer, J. Kauffman, J. Reepmeyer, C. Gryniewicz, W.Ye, D. Toler, L. Buhse, B. Westenberger. J. Pharm. Sci. 2009, 98,3540-3547.

9. H. Wu, C. Saez, M. Campana, E. Megehee, E. Wang. Anal. Chim. Acta. 2013, 804, 221-227.

10. S. Elci, D. Moyano, S. Rana, G. Tonga, R. Phillips, U. H. F. Bunz, V. Rotello. Chem. Sci. 2013, 4, 2076-2080.

11. E. Nyren-Erickson, M. Haldar, Y. Gu, S. Qian, D. Friesner, S. Mallik. Anal. Chem. 2011, 83, 598995.

12. R. B. Jagt, R. F. Gomez-Biagi, M. Nitz. Angew. Chemie. 2009, 48, 1995-7.

13. P. Muller-Graff, H. Szelke, K. Severin, R. Kramer. Org Biomol Chem 2010, 8, 2327-31. 
14. D. F. Moyano, V. M. Rotello. Langmuir 2011, 27 (17), 10376-85.

15. M. R. Pinto, K. S. Schanze. Proc. Natl. Acad. Sci. U.S.A. 2004, 101, 7505-7510.

16. B. S. Gaylord, A. J. Heeger, G. Bazan. Proc. Natl. Acad. Sci. U.S.A. 2002, 99, 10954-10957.

17. T. Nelson, C. O'Sullivan, N. Greene, M. Maynor, J. Lavigne. J. Am. Chem. Soc. 2006, 128, 56405641.

18. L. H. Wang, K. Y. Pu, J. Li, X. Y. Qi, H. Li, H. Zhang, C. H. Fan, B. Liu. Adv. Mater. 2011, 23, 4386-4391.

19. J. Pecher, S. Mecking, Chem. Rev. 2010, 110, 6260-6279.

20. S. W. Thomas, G. D. Joly, T. M. Swager. Chem. Rev. 2007, 107, 1339-1386.

21. C. L. Zhu, L. B. Liu, Q. Yang, F. T. Lv, S. Wang. Chem. Rev. 2012, 112, 4687-4735.

22. Y. J. Ko, E. Mendez, J. H. Moon. Macromolecules. 2011, 44, 5527-5530.

23. T. Vokatá, J. H. Moon. Macromolecules. 2013, 46, 1253-1259.

24. M. Twomey, Y. Na, Z. Roche, E. Mendez, N. Panday, J. He, J. H. Moon. Macromoleules. 2013, 46, 6374-6378.

25. To estimate GAG concentration levels in urine, we approximated molar concentrations based on previously reported concentration of total uronic acid excreted from normal (i.e, healthy) subjects. The value for normal subjects was reported as $\sim 8 \mathrm{mg} / \mathrm{L}$ (total uronic acid in $\mathrm{mg}$ per liter of urine) (see Y. Michelacci, R. Glashan, N. Schor. Kidney Int. 1989, 36, 1022-1028.). We converted to molarity based on the repeating unit of $\mathrm{HS}$ ( $\mathrm{MW}=\sim 665 \mathrm{~g} / \mathrm{mol}$ ), resulting in a value of $\sim 10 \mu \mathrm{M}$. HS was chosen because it has the highest repeating unit, therefore, resulting in the lowest concentration upon conversion. To demonstrate sensitivity of our system in a biological medium, we performed LDA at a concentration 100 times below (100 nM) the reported value for all GAGs.

26. V. Filipe, A. Hawe, W. Jiskoot. Pharm. Res. 2010, 27, 796-81.

27. C. C. You, O. R. Miranda, B. Gider, P. S. Ghosh, I. B. Kim, B. Erdogan, S. A. Krovi, U. H. F. Bunz, V. M. Rotello. Nat. Nanotechnol. 2007, 2, 318-323.

28. E. Mendez, J. H. Moon. Chem. Comm. 2013, 49, 6048-6050.

29. T. Vokatá, M. Twomey, E. Mendez, J. H. Moon. J. Polym. Sci., Part A: Polym. Chem. 2015, 53, 1403-1412. 


\section{CHAPTER IV}

Mitochondria-specific conjugated polymer nanoparticles

Reproduced in part with permission from Chemical Communications 2016, 52, 4910-4913

Copyright $(\mathbb{2} 2016$

Royal Society of Chemistry

Megan Twomey, ${ }^{a}$ Eladio Mendez, ${ }^{a}$ Rajesh Kumar Manian, ${ }^{a, b}$ Sunwoo Lee ${ }^{b *}$ and Joong Ho Moon ${ }^{a^{*}}$

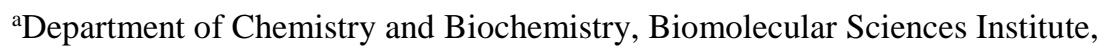

Florida International University, Miami, Florida, 33199

bepartment of Chemistry, Chonnam National University, Gwangju, South Korea 


\subsection{Abstract}

Biodegradable conjugated polymer nanoparticles (CPNs) were prepared for high mitochondria targeting in live cancer cells. The degradable CPNs are nontoxic and specifically localized to mitochondria of live tumor cells through macropinocytosis followed by intracellular degradation and trafficking.

\subsection{Introduction}

Conjugated polymer nanoparticles (CPNs) are intrinsic fluorescent soft materials fabricated by self-assembly of non-aqueous soluble conjugated polymers (CPs) in an aqueous solution. ${ }^{1,2}$ Owing to their excellent photophysical (i.e., high brightness and photostability) and biophysical properties (i.e., high cellular entry and nontoxicity), CPNs have recently gained much attention for microscopic live cell/tissue imaging, ${ }^{3-5}$ biological sensing, ${ }^{6,7}$ and nucleic acid delivery. ${ }^{8,9}$ By introducing non-conjugated degradable linkers along the conjugated backbones, biodegradable CPs were synthesized and exhibit similar fluorescent properties of fully conjugated CPs. ${ }^{10}$ The backbone modification with flexible linker affects the self-assembly properties of CPs, resulting in significantly changed cellular entry pathways. ${ }^{11}$

Despite successful cellular applications, the high molecular weights of CPs are not ideal for labelling or delivery to intracellular organelles due to inefficient diffusion and trafficking ability. For organelles with additional membranes such as nucleus and mitochondria, the necessary penetration of the organelle membranes further decreases the labeling and delivery efficiency. For the first time, presented here is a strategy to achieve efficient mitochondria labeling of live cells using intracellular degradation of CPNs. Biodegradable CPNs were designed by self-assembly of disulfide containing CPs. Upon endocytosis, CPNs were disassembled and degraded to low molecular weight, fluorescent conjugated oligomers (COs) that were efficiently trafficked to mitochondria (Fig. 4.1). The results support that biodegradable CPNs are promising materials for labeling and therapeutic delivery to mitochondria, which is an important therapeutic target given that mitochondria dysfunction is related to various diseases such as cancer, obesity, diabetes, and neurodegenerative disorders. ${ }^{12}$ 


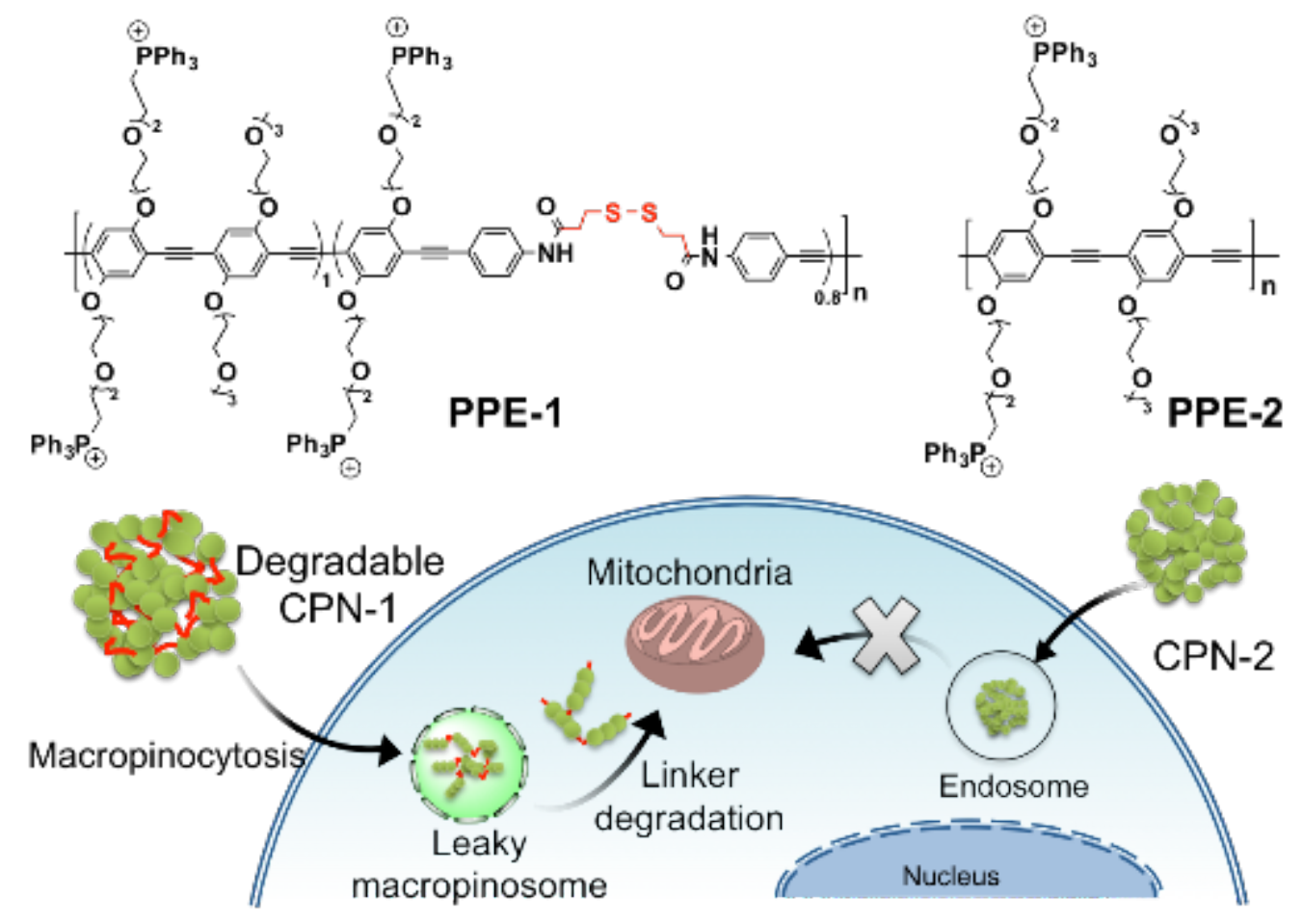

Figure 4.1. Chemical structures of PPEs with (PPE-1) and without (PPE-2) biodegradable linkers in the backbone and a schematic illustration of cellular entry and mitochondria localization of biodegradable CPNs. CPN-1 and CPN-2 were fabricated with the corresponding PPEs via self-assembly in water.

\subsection{Results and Discussion.}

Biodegradable poly(p-phenyleneethynylene)s (PPEs) were synthesized by polymerizing monomers including a disulfide-containing monomer (see experimental). Disulfide bonds are reducible under a high concentration of intracellular glutathione $(\sim 0.5-10 \mathrm{mM})^{13}$. To obtain the fluorescent properties comparable to a fully conjugated PPE, the amount of disulfide monomer in the PPE backbone was maintained less than 50\%. To improve mitochondria targeting function, triphenylphosphonium (TPP) salts were introduced by reacting the PPEs containing bromine side chains with triphenylphosphine (see experimental). TPP is a wellknown mitochondria-targeting small molecule and has been used for modifying small molecules, nanoparticles, and liposomes. ${ }^{14,} 15$ Proton and phosphorus nuclear magnetic resonance (NMR) spectroscopy confirmed the quantitative introduction of TPP salts (see experimental). The resulting PPE-1 $\left(M_{n}=9,300\right)$ and -2 $\left(M_{n}=16,000\right)$ were soluble in organic solvents such as dimethylsulfoxide (DMSO) and methanol. The ratio of repeating units of PPE-1 was calculated as 1.0:0.8 using proton NMR integration values, 
indicating that the average length of COs along PPE-1 backbone is around 5 repeating units (i.e., pentamer) or slightly larger.

CPNs were prepared by diluting TPP-modified PPEs in DMSO with an excess amount of water (i.e., final DMSO concentration of less than $1 \%$ ). Using fully conjugated PPEs, non-degradable CPNs were also fabricated to support the concept that the degradability of CPNs is crucial for high intracellular organelle targeting. The photophysical and physical properties of both CPNs were somewhat similar. Both CPNs exhibit broad emission spectra centered on $\sim 470 \mathrm{~nm}$. Using nanoparticle tracking analysis, the mean hydrodynamic diameters of CPN-1 and -2 were determined as $139.9 \pm 3.0$ and $152.7 \pm 6.7 \mathrm{~nm}$, respectively (see Figure S4.25 and S4.27). The difference in the average size is believed to associate with the size distribution of CPNs. Indeed, both CPNs exhibit very similar mode diameters (i.e., the diameter of the major population) of $92-93 \mathrm{~nm}$. Zeta potentials of CPN-1 and -2 were +24 and $+29 \mathrm{mV}$, respectively. Attempts to take transmission electron microscopic (TEM) images were not successful as CPNs heavily aggregated on a copper grid during sample preparations.

The metabolic activities of human cervical carcinoma (HeLa) cells incubated with biodegradable CPN-1 and non-degradable CPN-2, respectively, for overnight were monitored to evaluate the toxicity of CPNs. Interestingly, while degradable CPN-1 exhibits no viability inhibition up to $40 \mu \mathrm{M}$, non-degradable CPN-2 exhibits substantially high toxicity starting from $10 \mu \mathrm{M}$ (see Figure S4.35). To test whether the molecular weights of PPEs are associated with the toxicity, a low molecular weight $\left(M_{n}=6,960\right)$ non-degradable PPE (PPE-3) as synthesized by breaking the monomer stoichiometric balance in polymerization. The CPN fabricated with low molecular weight PPE (i.e., CPN-3) exhibits very similar toxicity to that of CPN-2, suggesting that the toxicity of CPN-2 is not related to the molecular weight of the corresponding PPE. Although further systematic investigations are necessary to better understand the backbone structure and the cellular toxicity relationship, it is speculated that the degraded oligomers containing thiols may contribute to the lower toxicity by reducing the level of reactive oxygen species. ${ }^{17}$

CPNs were taken up by live cells and found in the cytosolic compartment (Fig.4.2). Unlike CPN-2, which exhibits a characteristic punctuated pattern, the fluorescent image of CPN-1 was somewhat diffused along with some granular patterns (Fig. 4.2). The diffused pattern is indicative of disassembly of CPNs followed 
by degradation to COs via disulfide degradation under high intracellular glutathione concentration. Nondegraded CPNs were seen as punctuated dots. Nonetheless, the microscopic images are clearly different between biodegradable CPN-1 and non-degradable CPN-2. The possibility of pre-degradation of CPN-1 in extracellular environment followed by diffusion or uptake of COs by cells was ruled out, as disulfide reduction at the extracellular environment will be very inefficient due to lower glutathione concentration (i.e., one to three orders of magnitude lower). ${ }^{13}$
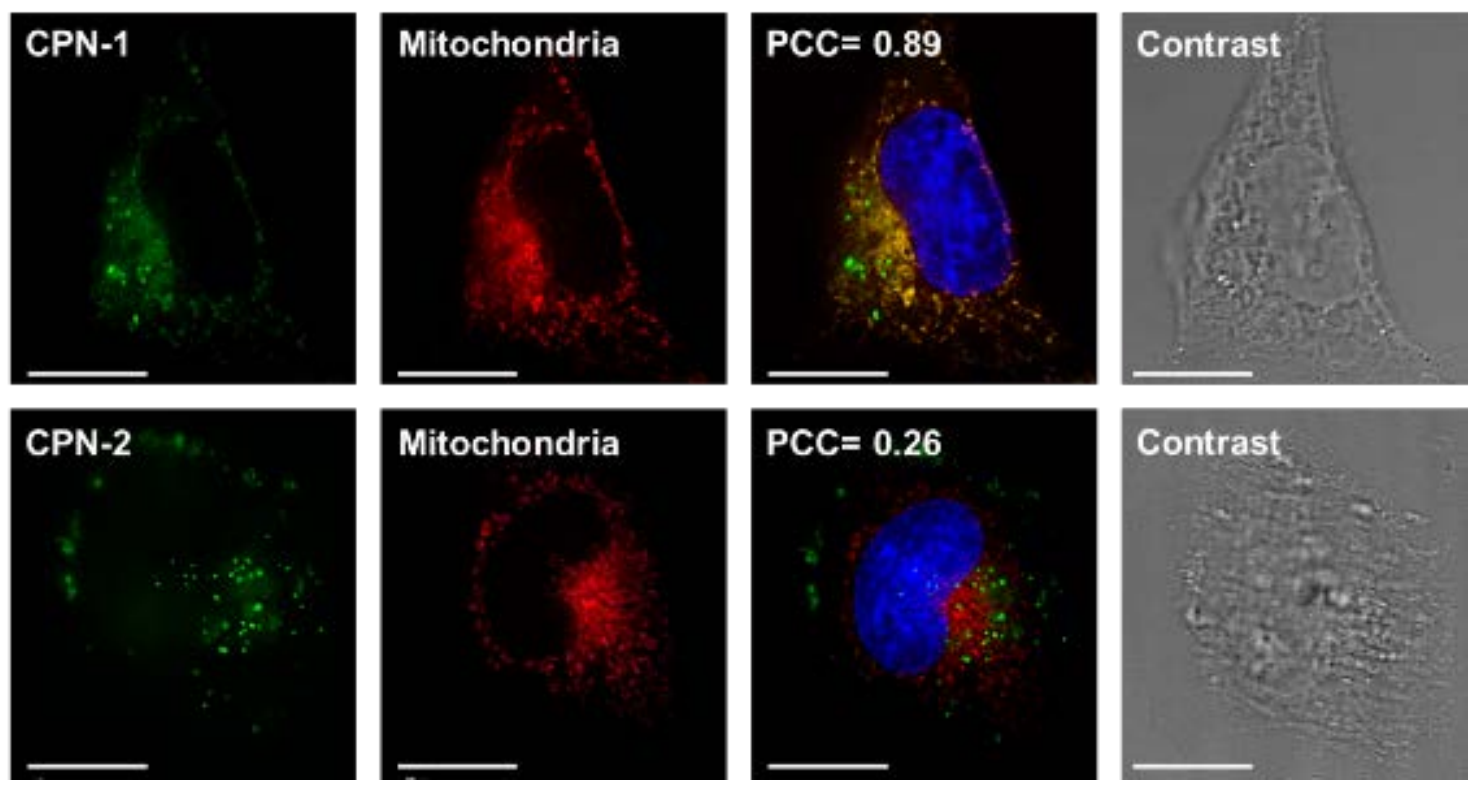

Fig. 4.2. Microscopic images of HeLa cells incubated with CPNs for $18 \mathrm{~h}$ followed by mitochondria (red) and nuclear (blue) staining. The scale bar is $20 \mu \mathrm{m}$.

The PPE stock concentration in DMSO is influential for CPN formation and thus for fluorescent microscopic imaging. The higher stock concentration, the more punctuated spots observed in the microscopic images (see Fig 4.3). As polymer concentration increases, the chances of interpolymer aggregation via $\pi$ - $\pi$ staking in a poor solvent increase. The punctuated spots are indicative of aggregated nondegraded CPNs. Attempts to degrade CPNs under high reducing conditions were not conclusive, assuming that the strength of interpolymer interactions in an aqueous environment are somewhat strong. Partial backbone degradation to induce de-aggregation of CPNs was speculated from slight blue shifts on both absorption and emission wavelengths upon treating CPNs with excess amount of glutathione (see Fig. 4.4). 


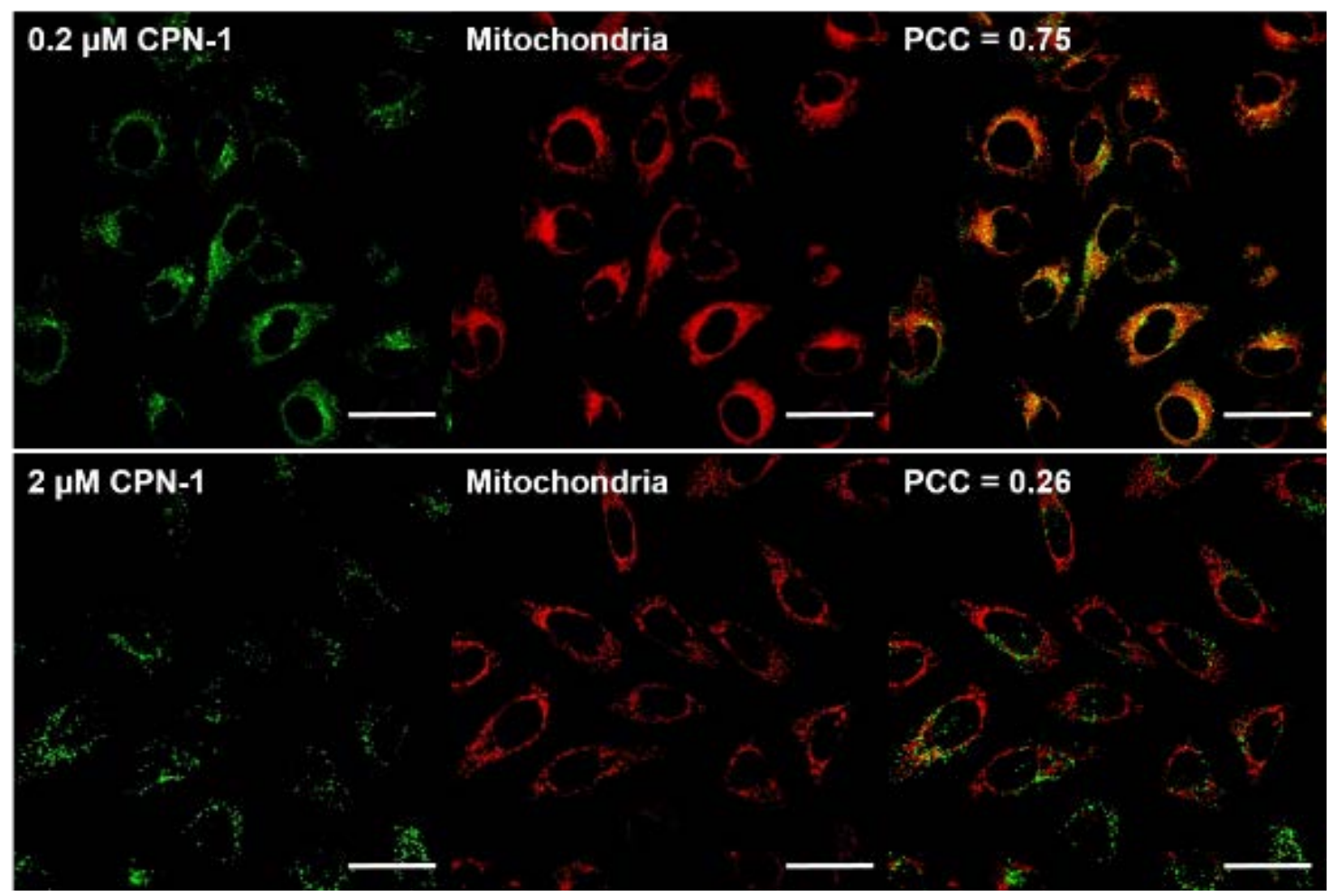

Fig. 4.3. Confocal microscopic images of HeLa cells incubated with CPN-1 $0.2 \mu \mathrm{M}$ (top row) and $2 \mu \mathrm{M}$ (bottom row) for $18 \mathrm{~h}$ followed by mitochondria (red) staining. Scale bar is $20 \mu \mathrm{m}$.

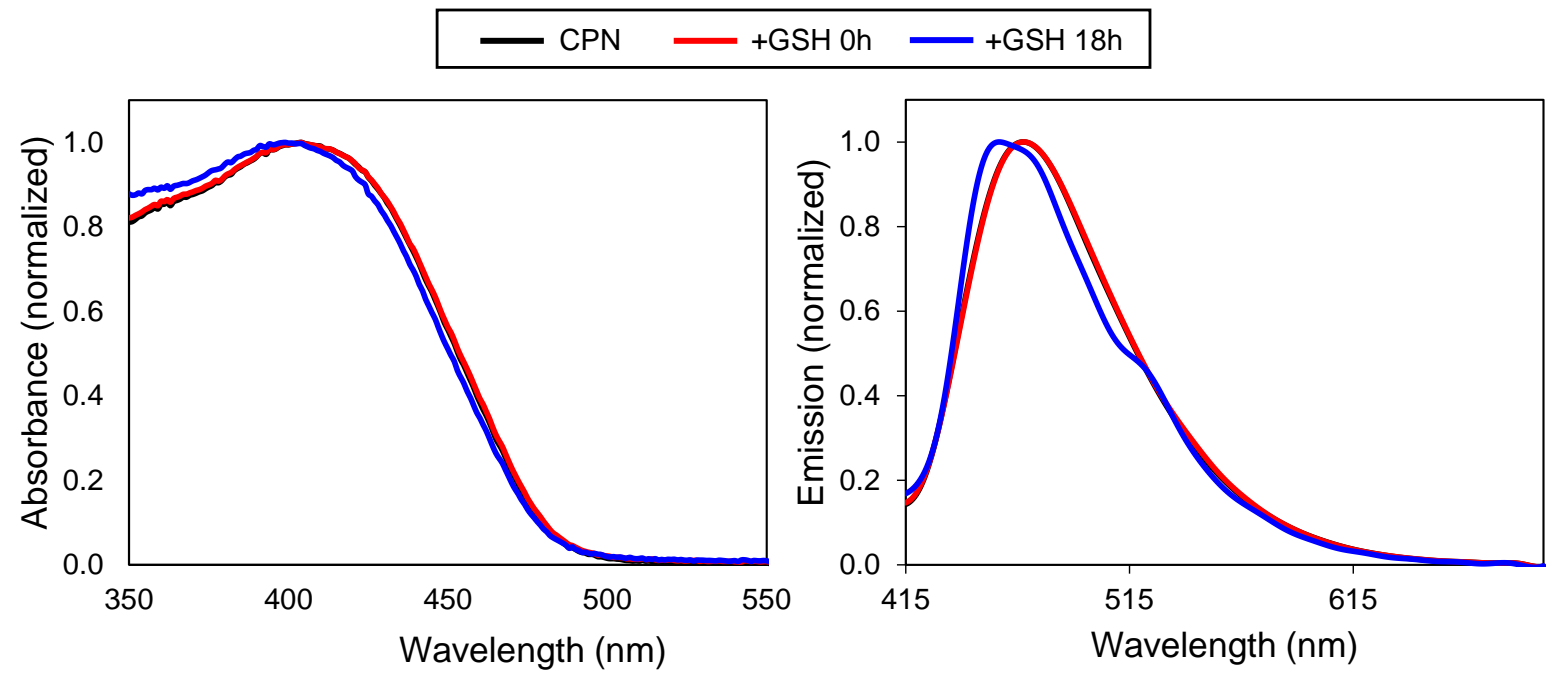

Fig. 4.4. Absorption (left) and emission (right) spectra of CPN-1 treated with $10 \mathrm{mM}$ glutathione (GSH) for 0 and $18 \mathrm{~h}$ at $37^{\circ} \mathrm{C}$.

For analyzing subcellular localizations of CPNs, various organelles (i.e., nucleus, Golgi, endosome, and mitochondria) were post-stained using commercially available dyes. The Pearson's Correlation Coefficient (PCC) was used to quantitatively evaluate co-localization. ${ }^{18}$ PCC scores of 0 and 1 correspond to uncorrelated 
and perfectly linearly correlated colors, respectively. Mean PCC scores from three independent images of an entire cell were selected and analyzed to increase the analysis objectivity. CPN-1 exhibited high mitochondrial co-localization (PCC $\sim 0.8$ ), while minimal endosome ( $\sim .3)$ and Golgi apparatus ( 0.2) colocalizations were observed. Meanwhile, CPN-2 showed high endosomal co-localization ( $\sim 0.7)$ with minimal mitochondrial and Golgi apparatus co-localization. Non-degradable CPN-3, which was fabricated with low molecular weight PPE-3, also exhibits punctuated staining pattern with low mitochondria localization $(\sim 0.4)$, supporting that the degradability of CPNs is an important factor increasing mitochondria trafficking (see Fig. 4.5).

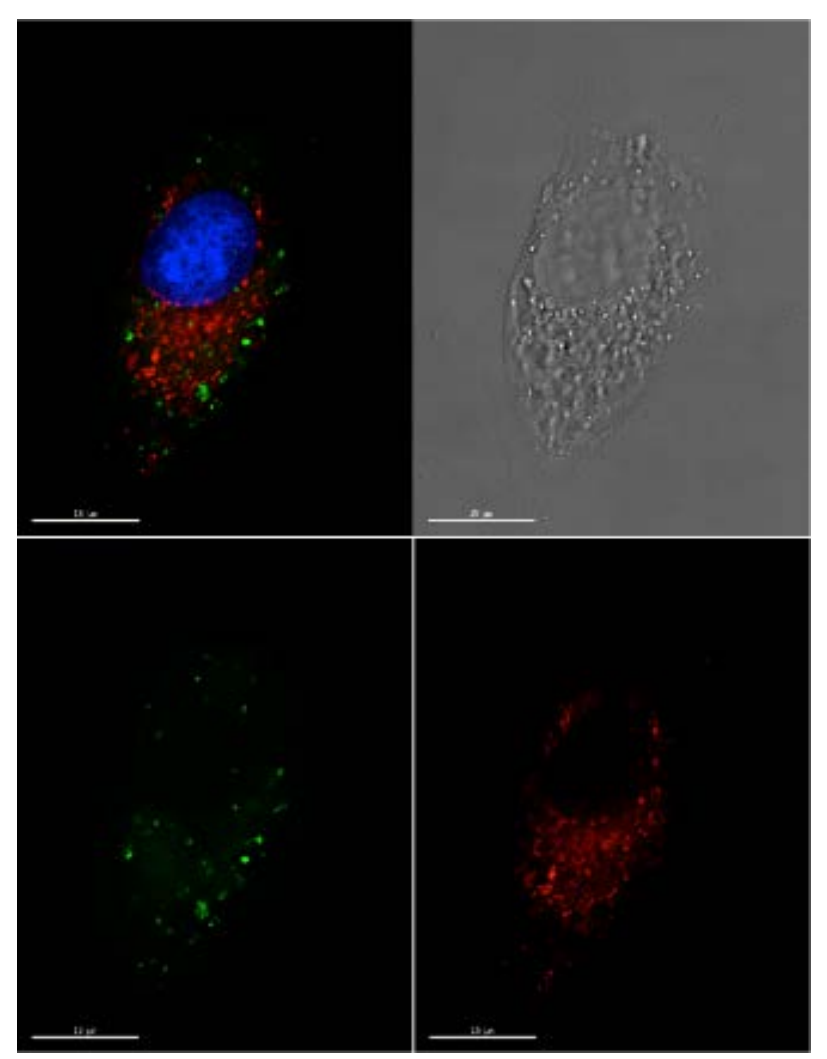

Fig. 4.5. Microscopic images of HeLa cells incubated with CPN-3 for $18 \mathrm{~h}$ followed by mitochondrial (red) and nuclear (blue) staining. The scale bar is $15 \mu \mathrm{m}$.

The presence of punctuated spots in microscopic images and inefficient degradation of CPN-1 in a highly reducing environment imply that CPNs are somewhat hydrophobic despite of the charged TPP groups. To increase the hydrophilicity, CPNs were further complexed with an anionic linear polysaccharide, hyaluronic acid (HA). When the same biodegradable CPN-1 was complexed with HA (CPN-1/HA) followed by 
incubation with HeLa cells for overnight, the mitochondria imaging pattern was improved substantially with reduced punctuated spots (Fig. 4.6). As shown in Fig.4.6, CPN-1/HA exhibits characteristic mitochondrial granular patterns and high PCC scores, while no changes were observed from CPN-2/HA complex. Nondegradable CPN exhibit punctuated fluorescent image patterns regardless of HA complexation.

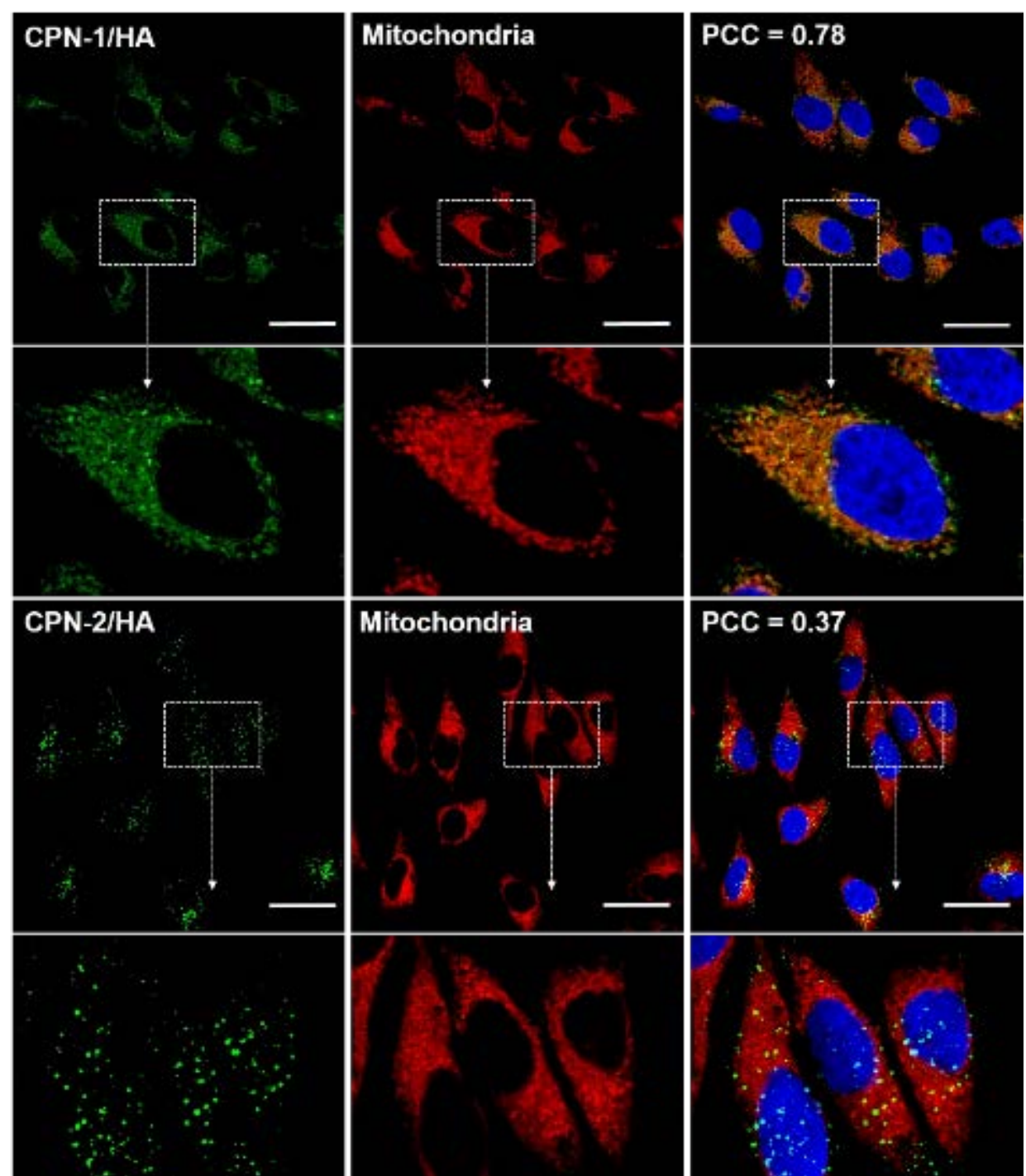

Fig. 4.6. Confocal microscopic images of HeLa cells incubated with CPN/HA (green) for $18 \mathrm{~h}$ followed by mitochondria (red) and nucleus (blue) staining. The scale bar is $20 \mu \mathrm{m}$.

To further confirm that CPN-1 indeed trafficked to mitochondria, live cells incubated with CPNs were treated with a commercial mitochondria isolation kit followed by centrifugation to isolate a mitochondria fraction. Fluorescence spectroscopic analysis of the mitochondrial fraction of live HeLa cells clearly supports the high mitochondrial localization of CPN-1. As shown in Fig. 4.7, fluorescent signals were exclusively observed 
from the mitochondria fraction of live HeLa cells incubated with CPN-1. CPN-2 exhibited high cytosolic fluorescent intensity compared to the mitochondria fraction, supporting the data from microscopic image analysis.

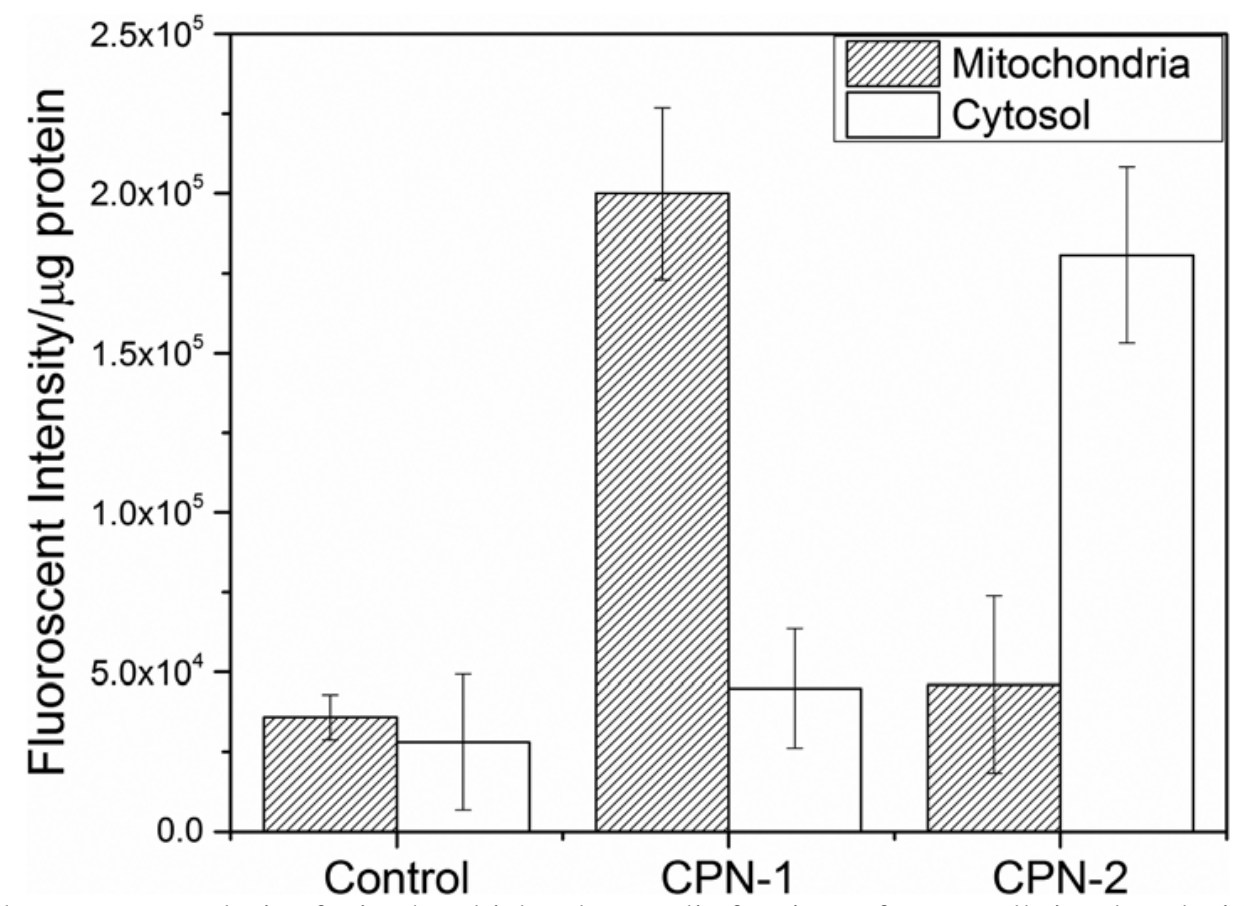

Fig. 4.7. Fluorescence analysis of mitochondrial and cytosolic fractions of HeLa cells incubated with CPNs. CPN-1 was found at the mitochondrial fraction, while CPN-2 was found mainly in the cytosolic fraction. The error bar represents \pm standard deviation $(\mathrm{n}=3)$.

This dramatic subcellular localization difference between CPNs with the same side chains (i.e., TPP) implies that self-assembly structures of CPNs possibly influence cellular entry routes and the intracellular fates. Previously, it was found that CPs with flexible linkers tend to form aggregates with high interpolymer interactions compared with CPs without the flexibility. ${ }^{19}$ Because of the flexible disulfide containing units, the surface properties of CPN-1 will be different and influential for cellular interactions and subsequent cellular entry. To test endocytosis pathways, live HeLa cells pretreated with pharmacological inhibitors of various endocytosis pathways were incubated with CPNs for $2 \mathrm{~h}$ and mean fluorescence intensity of live cells was monitored by flow cytometry. ${ }^{20}$ As shown in Fig. 4.8, CPNs use various endocytosis pathways to enter HeLa cell, determined by the reduced uptake of CPNs under the inhibitors tested. In addition to caveolaemediated endocytosis (CvME), uptake of CPN-1 via macropinocytosis (MPC) was also noticeably reduced. Although further mechanistic and trafficking studies are needed, it is speculated that the high mitochondria 
localization of CPN-1 is closely related to a series of cellular processes of MPC internalization of CPN-1, better escape of macropinosomes, degradation of disulfide bonds to generate low molecular weight COs, and increased diffusion for trafficking to mitochondria.

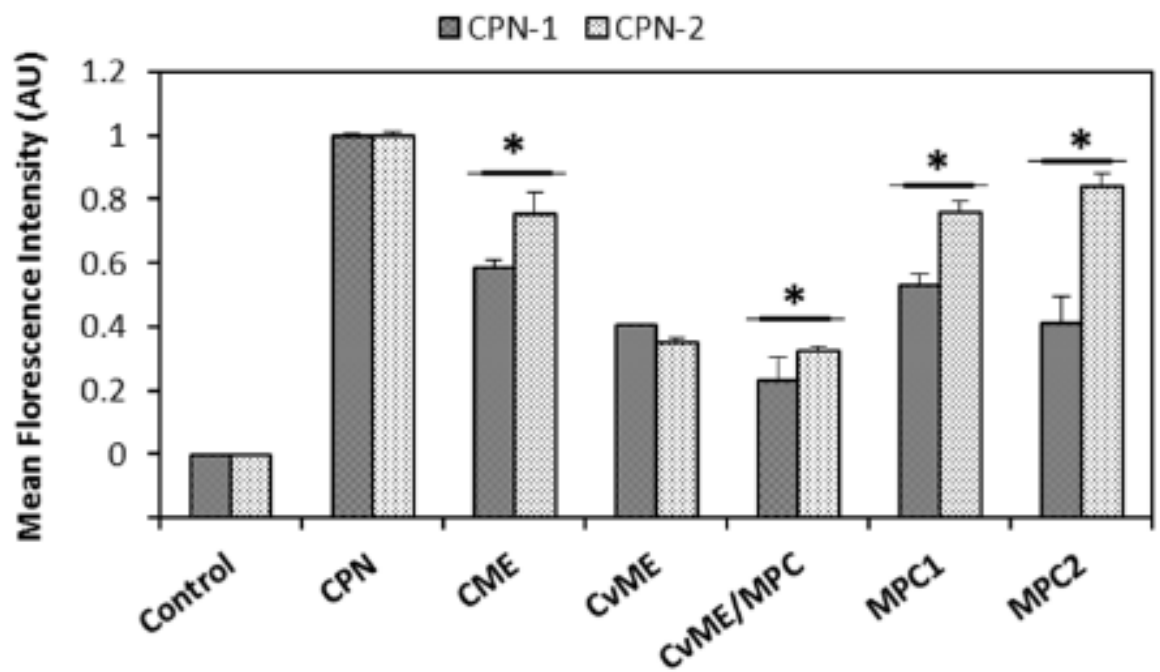

Fig. 4.8 Endocytosis inhibition study under pharmacological inhibitors treatments. Mean fluorescence intensity of HeLa cell (Control), CPNs only (CPN), chlorpromazine (24.0 mM, CME), genistein (0.21 mM, CvME), methyl- $\beta$-cyclodextrin (1.00 mM, CvME/MPC), LY294002 (0.12 mM, MPC1), and cytochalasin D (0.04 mM, MPC2) were measured using flow cytometry. Error bars represent standard deviation. ${ }^{*} \mathrm{p}<0.05$

To determine whether the observed $\mathrm{CPN}-1$ mitochondrial specificity is related to the degradation kinetics, endosome and mitochondrial co-localizations were measured as a function of incubation time. Subcellular localization was monitored by fluorescent microscopic imaging, with CPNs incubated for 3, 6, 12, and $18 \mathrm{~h}$ in live HeLa cells. PCC scores between CPNs and mitochondrial or endosomal markers were measured and averaged from three independent trials (Fig. 4.9). CPN-1 exhibited a time-dependent mitochondrial and endosomal co-localization. As incubation time increased, CPN-1 localized more strongly toward the mitochondria, with almost exclusive mitochondrial localization at $18 \mathrm{~h}$ incubation. CPN-2, meanwhile, did not show time-dependent co-localization, exhibiting no significant change in mitochondrial or endosomal localization as incubation time increased. Although $\mathrm{CPN}-2$ has high concentration of mitochondria targeting TPPs, the non-degradability, large molecular weight, and poor endosome escaping and diffusion efficiency limit mitochondria targeting. The time-dependency of CPN-1 on co-localization supports that intracellular degradation of biodegradable linkers play a significant role in mitochondrial trafficking of TPP functionalized COs. 

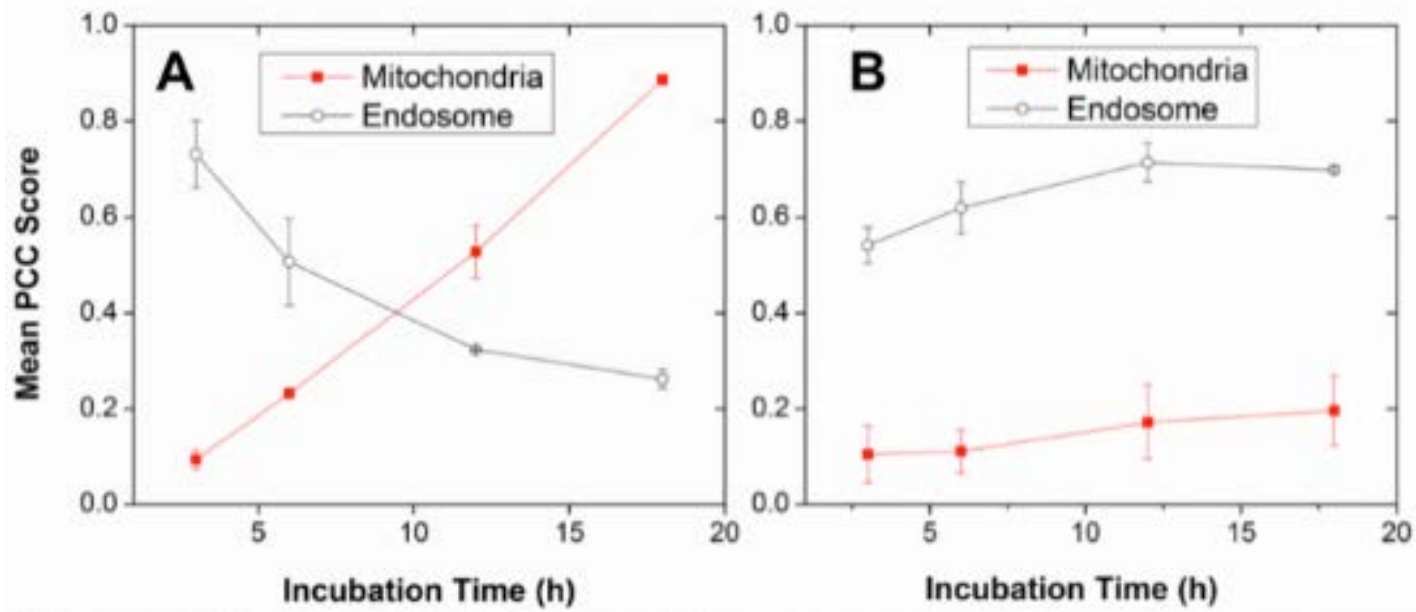

Fig. 4.9. Time-course localizations of $\mathrm{A}) \mathrm{CPN}-1$ and $\mathrm{B}$ ) $\mathrm{CPN}-2$ on endosomes (open circles) and mitochondria (filled circles). Subcellular localization of $\mathrm{CPN}-1$ changes from endosome to mitochondria, while CPN-2 remains constant regardless of incubation time. Error bars represent standard deviation from $\mathrm{n}$ $=3$.

\subsection{Conclusion}

In conclusion, a new strategy to achieve a unique mitochondrial-specific localization of CPs was presented.

It was demonstrated that disulfide-containing CPNs exhibit active cellular internalization as polymer nanoparticles via a variety of endocytosis, and undergoes degradation to low molecular weight oligomers that were efficiently trafficked to target mitochondria. The concept demonstrated here will lead to the development of novel CP-based materials for improved intracellular sensing, labeling, and potential therapeutic delivery.

\subsection{Outlook}

This chapter presented a strategy for mitochondrial targeting that was dependent on the biodegradability and flexibility of the conjugated backbone. With mitochondria-specificity, CPNs could be used for sensing and delivery applications by incorporating a reactive oxygen species (ROS)-sensitive functionality. Since the writing of this chapter, a series of non-flexible-containing TPP copolymers with varying molecular weight and a series of flexible TPP-containing copolymers with varying flexible unit incorporation were synthesized and screened for mitochondrial-specificity. Preliminary results indicated that the presence of the flexible linker and at relatively higher percentage ( $>40 \%$ ) was crucial for mitochondrial localization. This is could be 
due to the hydrophobicity of the polymer backbone, which causes aggregation in aqueous environment. If the polymer aggregates, the availability of the degradable linker in the backbone would be compromised and degradation by glutathione would be difficult. A series of TPP-containing polymers that also incorporated the hydrogen peroxide-sensitive moiety, aryl boronate, and degradable linker (Scheme 4.1). However, due to the hydrophobicity of this group and in combination with the bulky TPP group, dense aggregation was observed both in photophysical properties (red shifted spectra) and decreased emission intensity. The dense aggregation led to non-specific binding to the cellular membrane, and low mitochondria localization, for both linker and non-linker CPNs.

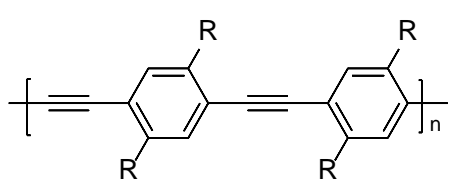

PPE

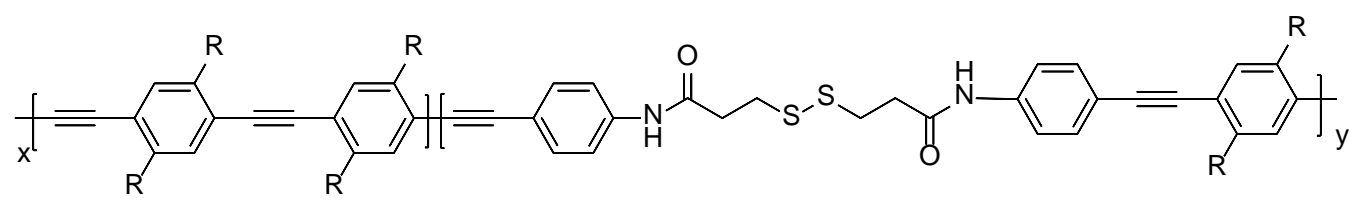

PPE-L

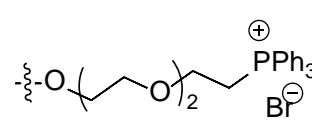

R1

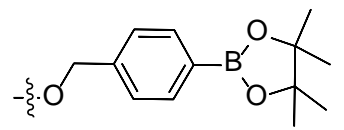

R2

Scheme 4.1: TPP (R1)-containing polymers with (PPE-L) and without (PPE) biodegradable linker and boronate-moiety (R2).

To address these issues, more in-depth studies on the effect of the flexible linker for subcellular targeting are needed. In addition, improving the hydrophilicity of the TPP-containing CPNs would provide information as to how polymer solubility, CPN aggregation, and cellular uptake/trafficking can be modulated.

\subsection{Experimental}

Dr. Rajesh Kumar completed polymer synthesis and characterization. Dr. Eladio Mendez completed timecourse imaging and flow cytometry experiments. 


\subsubsection{General Information}

Chemicals, including solvents, were purchased from Fisher Scientific and used as received. Deuterated solvents were purchased from Cambridge Isotope Laboratories (Cambridge, MA). UV-vis spectra were recorded using a Varian Cary 50 Bio spectrophotometer. Fluorescence spectra were obtained using a FluoroLog-3 Spectrofluorometer (Jobin Yvon/Horiba). 9, 10-diphenylanthracene (QY = 1.0) in cyclohexane was used as a fluorescence standard for QY determination. The average molecular weight $\left(\mathrm{M}_{\mathrm{n}}\right)$ and polydispersity (PDI $=M_{w} / M_{n}$ ) of the polymers were determined by gel permeation chromatography (GPC) against polystyrene standards using a Shimadzu high performance liquid chromatography (HPLC) system fitted with PLgel $5 \mu \mathrm{m}$ MIXED-D columns and SPD-20A ultraviolet-visible (UV-vis) detector. Nuclear magnetic resonance (NMR) spectra were recorded on a $400 \mathrm{MHz}$ Avance Bruker NMR spectrometer. ${ }^{1} \mathrm{H}$ and ${ }^{13} \mathrm{C}$ NMR chemical shifts are given in ppm relative to $\mathrm{Si}\left(\mathrm{CH}_{3}\right)_{4}$, with the solvent resonance used as an internal reference. $1 \mathrm{H}$ NMR on the scale based on the middle peak $(\delta=2.50 \mathrm{ppm})$ of the dimethyl sulfoxide DMSO$\mathrm{d}_{6}$ solvent as an internal standard or $7.26 \mathrm{ppm}$ for chloroform $\left(\mathrm{CDCl}_{3}\right)$ experiments. 31P NMR chemical shifts are reported in ppm relative to $\mathrm{H3PO}$. The mass spectrometric data were obtained at the mass spectrometry facility of Florida International University. Analytical thin layer chromatography (TLC) was performed on TLC Silica gel 60 F254. The TLC plates were visualized by shortwave (254 nm) or longwave (360 nm) UV light. Flash chromatography on silica gel (230-400 mesh) was performed. Fourier transform infrared (FT-IR) spectrum was obtained on a PerkinElmer Spectrum 100 FT-IR Spectrometer; fine powder sample was mounted on an attenuated total reflection cell.

\subsubsection{Monomer Synthesis}

Scheme S4.1: Synthetic route to M1<smiles>Oc1cc(I)c(I)cc1O</smiles>

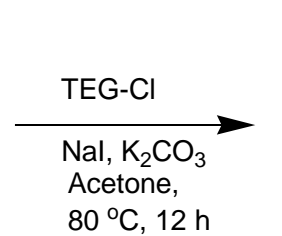

$80^{\circ} \mathrm{C}, 12 \mathrm{~h}$

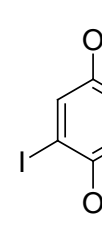<smiles>COc1ccc(OC)cc1</smiles><smiles>COCCOCCO</smiles>

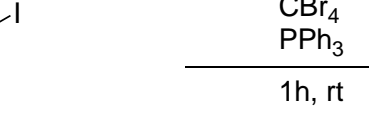<smiles>CCOCCOCCO</smiles> 


\subsubsection{Synthesis of compound $\mathbf{1}^{20}$}

1, 4-Dihydroxy-2, 5-diiodobenzene (5.00 g, $13.80 \mathrm{mmol})$ was dissolved in acetone $(20 \mathrm{~mL})$ and the resulting solution was added slowly to a stirred suspension of $\mathrm{K}_{2} \mathrm{CO}_{3}(7.60 \mathrm{~g}, 55.25 \mathrm{mmol})$ and $\mathrm{NaI}$ (8.30 g, 55.25 mmol). The reaction mixture was allowed to stir for $10 \mathrm{~min}$, followed by the slow addition of triethylene glycol monochlorohydrin (TEG-Cl) $(5.80 \mathrm{~g}, 34.53 \mathrm{mmol})$. The reaction was heated to $80{ }^{\circ} \mathrm{C}$ for $12 \mathrm{~h}$. The solution was cooled to room temperature, the solvent was removed, and the resulting slurry was re-dissolved in $\mathrm{CH}_{2} \mathrm{Cl}_{2}(100 \mathrm{~mL})$. The solution was extracted with water $(100 \mathrm{~mL} \mathrm{x} 3)$ and the organic fractions were collected and concentrated in vacuo until an oily residue remained. The crude product was purified by silica gel chromatography (95:5 EA/Hexane). The product was off-white solid. Yield: 5.80 g (67.0\%). ${ }^{1} \mathrm{H}$ NMR (400 MHz, $\mathrm{CDCl}_{3}$ ): $\delta 7.24$ (s, 2H), 4.09- 4.15 (t, 4H), 3.86-3.92 (t, 4H), 3.77-3.82 (m, 4H), 3.69-3.76 (m, 8H), 3.60-3.66 (m, 4H), 2.29 (br, 2H); ${ }^{13} \mathrm{C}$ NMR (100 MHz, $\left.\mathrm{CDCl}_{3}\right): \delta 153.51,123.21,86.52,72.51,71.22$, 70.54, 70.28, 69.66, 61.82.

\subsubsection{Synthesis of monomer $\mathrm{M1}^{21}$}

A suspension of compound 1 (5.00 g, $7.98 \mathrm{mmol})$ was dissolved in $25 \mathrm{~mL}$ of DCM. Carbon tetrabromide (8.00 g, $23.95 \mathrm{mmol}$ ) and triphenyl phosphine (12.60 g, $23.95 \mathrm{mmol}$ ) were added slowly to the reaction mass. The mixture was stirred at room temperature for $2 \mathrm{~h}$. The solvent was removed and the crude product was purified by column chromatography (1: 4 EA / Hexane). The product was white solid. Yield: $5.30 \mathrm{~g}(81.2 \%)$. ${ }^{1} \mathrm{H}$ NMR (400 MHz, $\mathrm{CDCl}_{3}$ ): $\delta$ 7.30 (s, 2H), 4.10-4.15 (t, 4H), 3.89-3.92 (t, 4H), 3.85-3.87 (t, 4H), 3.68-3.76 (m, 4H), 3.60-3.65 (m, 4H), 3.45-3.51 (m, 4H). $\left.{ }^{13} \mathrm{C} \mathrm{NMR} \mathrm{(100} \mathrm{MHz,} \mathrm{CDCl}_{3}\right): \delta 153.37,123.49,86.47,71.30$, 71.19, 70.71, 70.33, 69.70, 30.42 .

Scheme S4.2: Synthetic route to M2

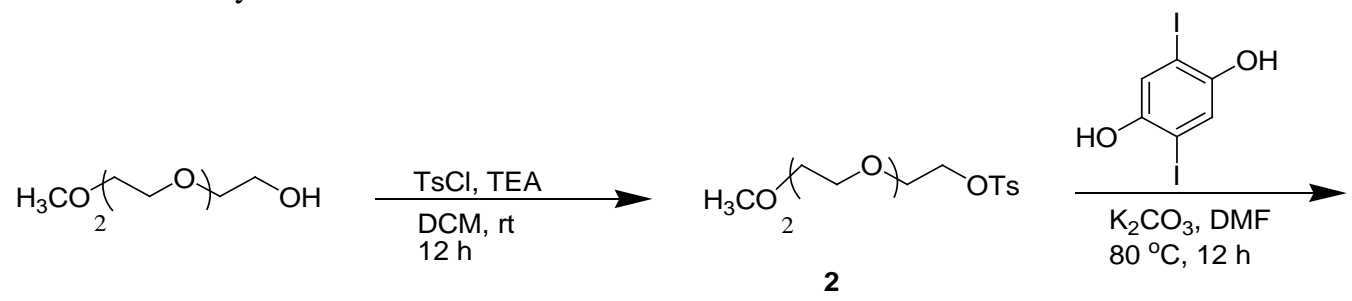




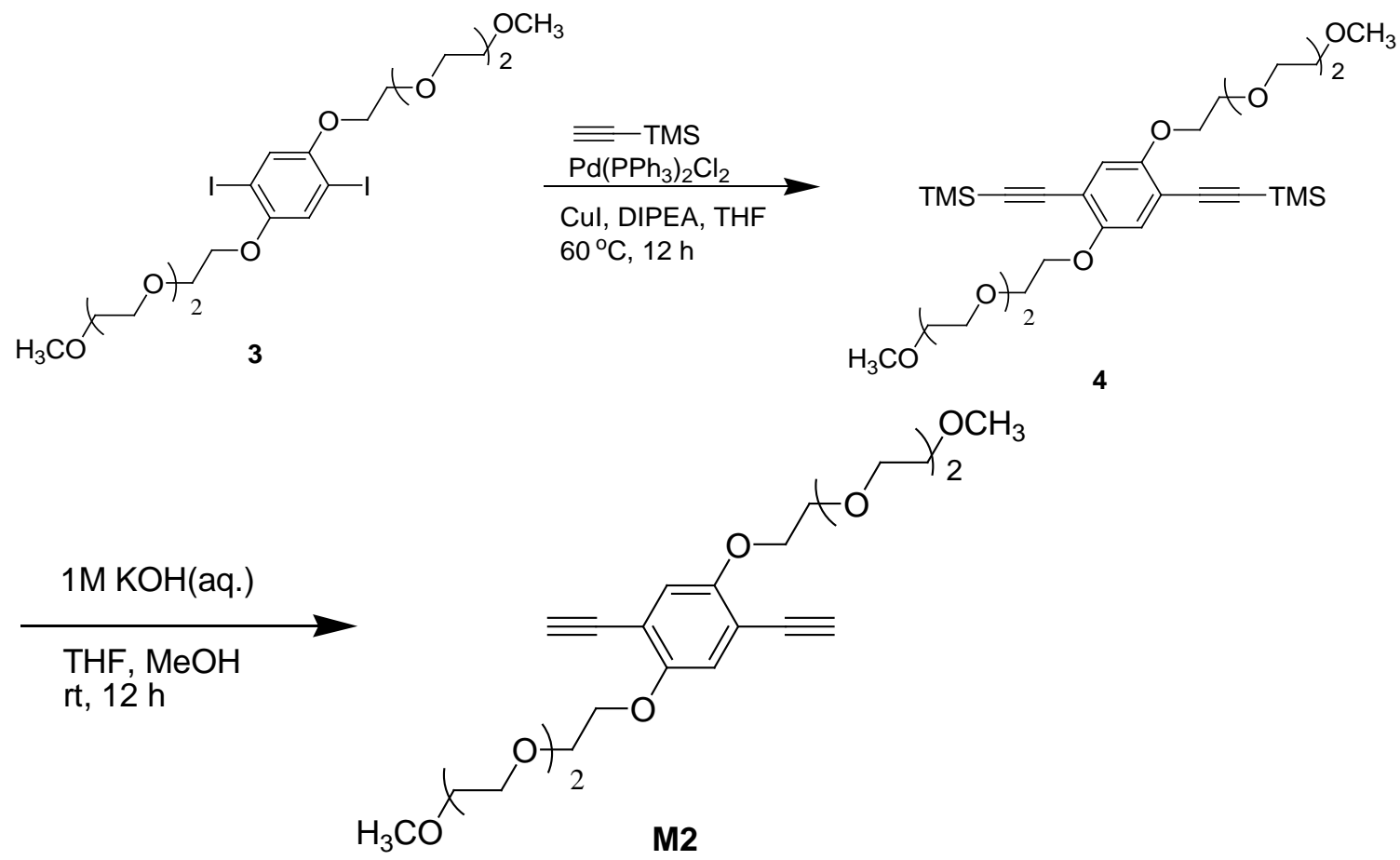

Monomer 2 (M2) was synthesized according to literature procedure (Scheme S4.2). ${ }^{22}$

Scheme S4.3: Synthetic route to M3

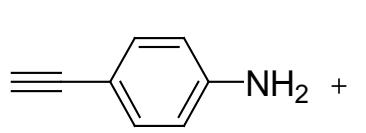

5<smiles>O=C(O)CCSSCCC(=O)O</smiles>

6
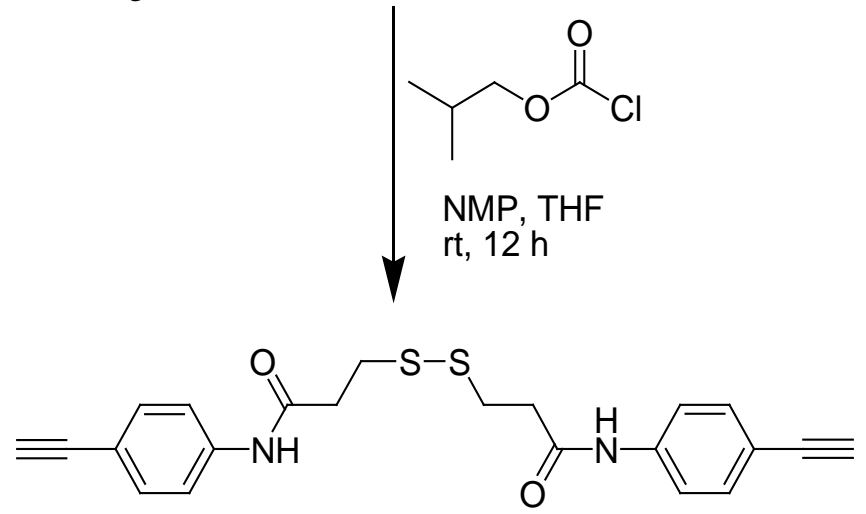

M3

\subsubsection{Synthesis of monomer M3}

3,3'-Dithiodipropionic acid, 6 (1.00 g, $4.76 \mathrm{mmol})$ was dissolved in anhydrous THF (20 $\mathrm{mL}$ ) in a round bottom flask, which was evacuated and filled with nitrogen. $N$-methylmorpholine $(0.90 \mathrm{~g}, 9.52 \mathrm{mmol})$ was added, and the suspension was allowed to stir until the entire solid was dissolved. Isobutyl chloroformate 
(1.30 g, $9.52 \mathrm{mmol}$ ) was added, and the mixture was allowed to stir at room temperature for 15 min. A solution of 4-ethynylaniline, 5 (1.10 g, $9.52 \mathrm{mmol})$ in anhydrous THF (10 mL) was prepared under a $\mathrm{N}_{2}$ atmosphere and transferred into the reaction flask using a cannula. The reaction was allowed to proceed overnight, after which the reaction mixture was filtered. The filtrate was concentrated in vacuo and the resulting solid was precipitated overnight from the DCM / diethyl ether solvent system. Yield: 0.70 g (36.0\%). ${ }^{1} \mathrm{H}$ NMR (400 MHz, DMSO): $\delta 10.20$ (s, 2H), 7.50-7.61 (d, 4H), 7.39-7.41 (d, 4H), 4.07 (s, $\left.2 \mathrm{H}\right)$, 2.99-3.03 (t, 4H), 2.74-2.77 (t, 4H). ${ }^{13} \mathrm{C}$ NMR (100 MHz, DMSO): $\delta 169.53,139.68,132.36,118.94,116.12$, 83.49, 79.65, 36.08, 33.38. HRMS (ESI): Calc for $\mathrm{C}_{22} \mathrm{H}_{20} \mathrm{~N}_{2} \mathrm{O}_{2} \mathrm{~S}_{2}[\mathrm{M}+\mathrm{Na}]^{+}$: 431.0858; found [M+Na] $]^{+}$: 431.0801.
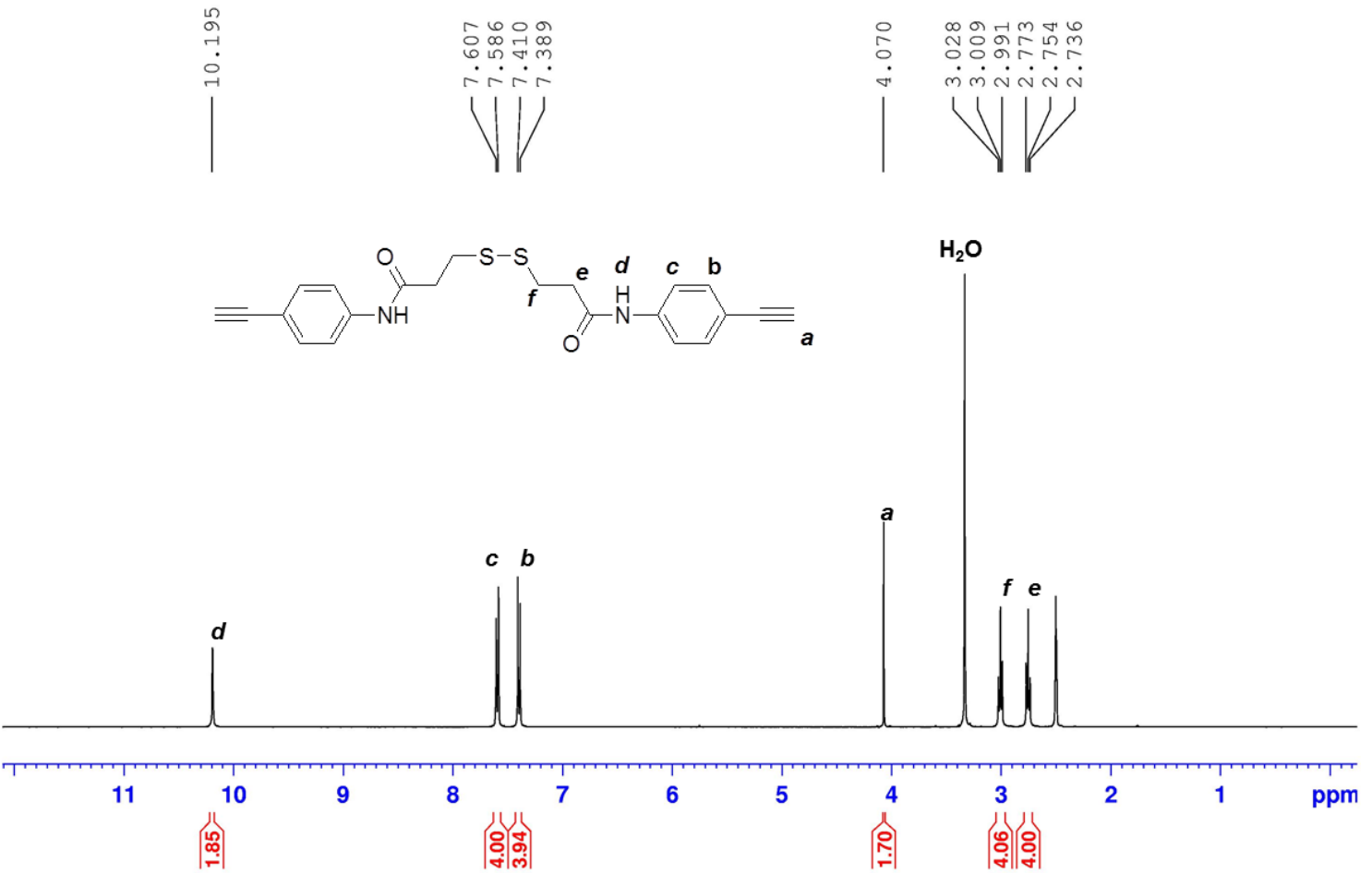

Figure S4.1. ${ }^{1} \mathrm{H}$ NMR of $\mathbf{M} 3$ in DMSO- $\mathrm{d}_{6}$ 

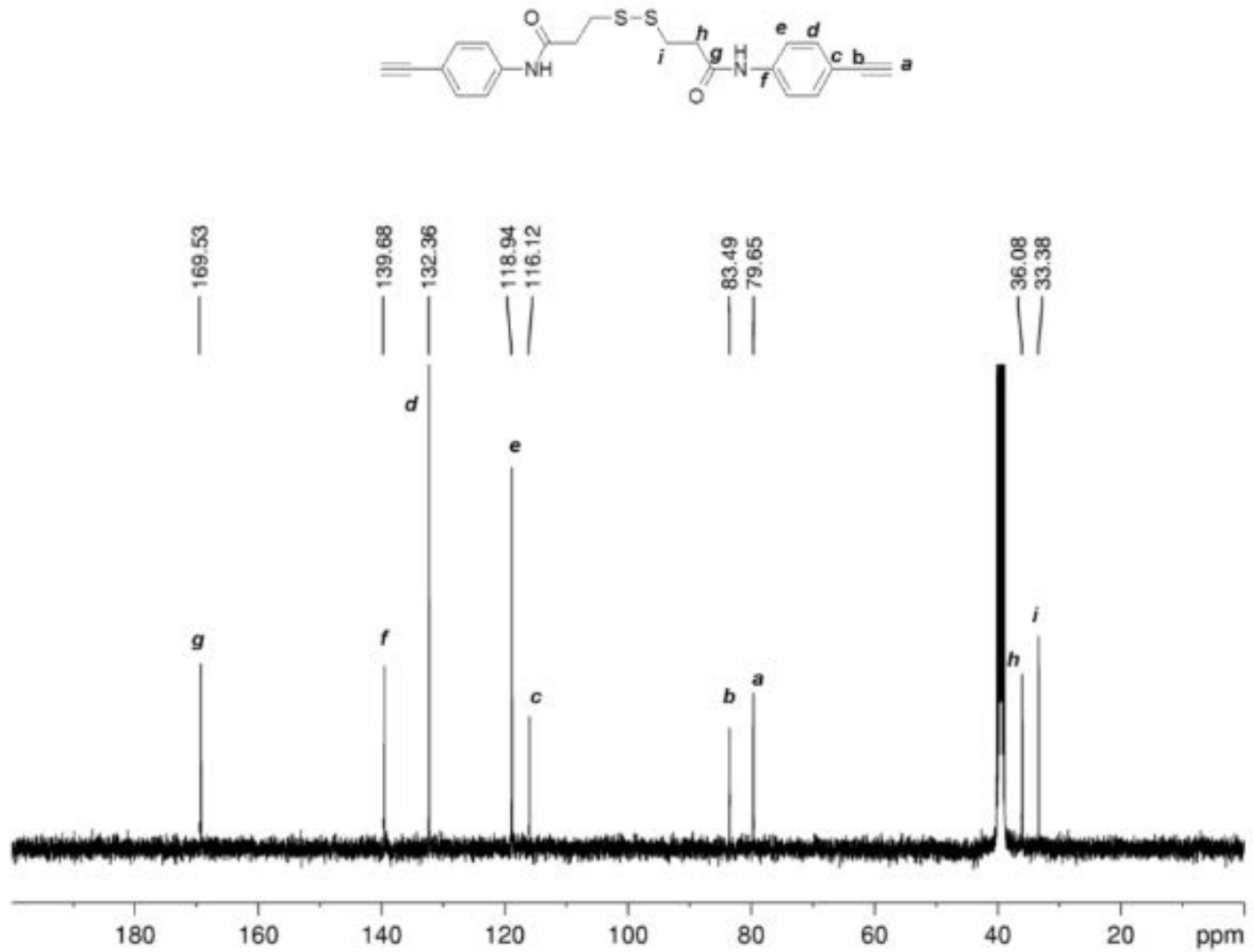

Figure S4.2. ${ }^{13} \mathrm{C}$ NMR of M3 in DMSO-d 6

\subsubsection{Polymer Synthesis}

General Procedure. A Schlenk flask was charged with monomer M1 (1.00 eqiv.), M2 (0.5 eqiv.), M3 (0.5 eqiv.), $\mathrm{Pd}\left[\left(\mathrm{PPh}_{3}\right)_{2} \mathrm{Cl}_{2}\right]$ (0.10 equiv.), and $\mathrm{CuI}$ (0.05 equiv.). The Schlenk flask was evacuated and filled with $\mathrm{N}_{2}$ and solution of anhydrous dimethylformamide $(4 \mathrm{~mL})$ and diisopropylamine $(1 \mathrm{~mL})$ was degassed, and $2.5 \mathrm{~mL}$ of the mixed solution was transferred to the Schlenk flask using a cannular needle. The reaction was allowed to stir at room temperature for $12 \mathrm{~h}$. The solution was then added drop wise to methanol, resulting in precipitation. After centrifugation (3 min, $4000 \mathrm{rpm}$ ) the supernatant was decanted, and the precipitate was redissolved in DCM (1 mL) for further purification. 


\subsubsection{Synthesis of PPE-1}

Scheme S4.4: Synthetic route to PPE-1
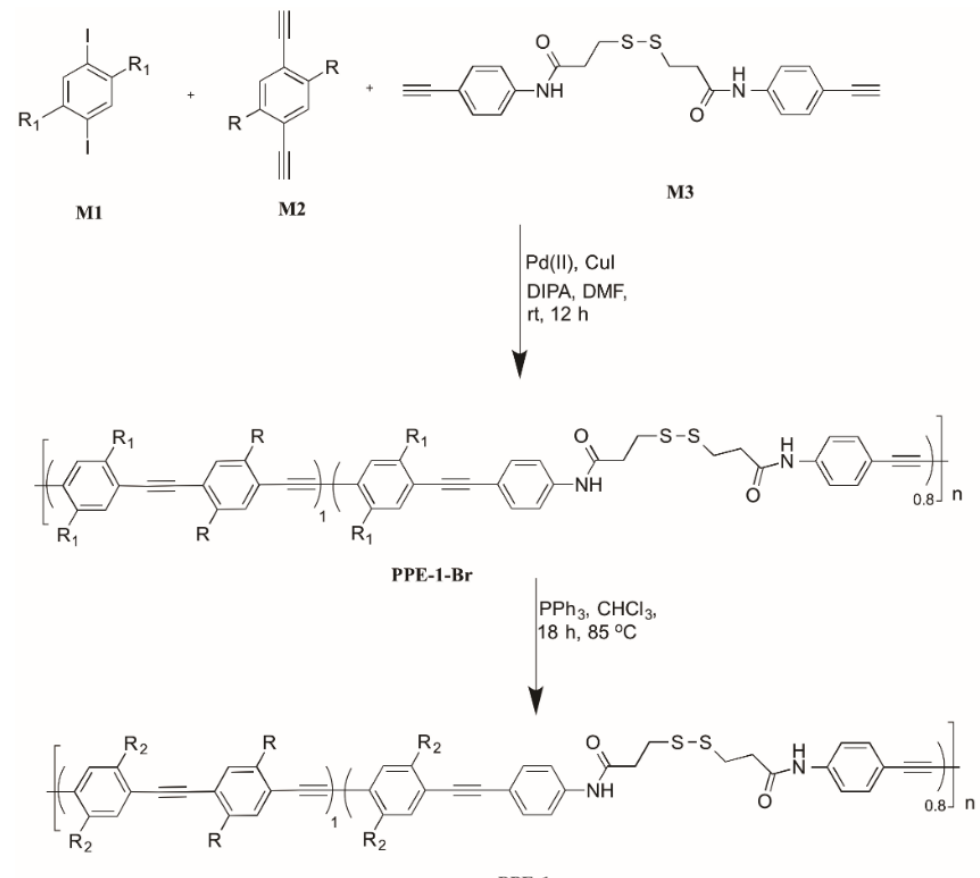

PPE-1

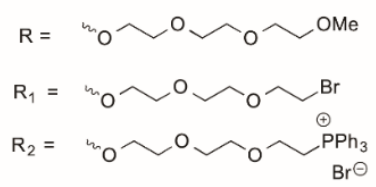

PPE-1-Br. Using the general procedure described above, the polymerization of monomer M1 (100 mg, 0.133 mmol), M2 (30 mg, $0.066 \mathrm{mmol})$ and $\mathbf{M} 3$ (28 mg, $0.066 \mathrm{mmol}$ ) was carried out in presence of $\mathrm{Pd}\left[\left(\mathrm{PPh}_{3}\right)_{2} \mathrm{Cl}_{2}\right]$ (9.3 mg, $0.013 \mathrm{mmol}$ ), and CuI (1.3 mg, $0.007 \mathrm{mmol}$ ). The resulting mixture was purified by precipitation in methanol (x3) Yield: 105 mg (42.5 \%). An aliquot was redissolved in THF, and the molecular weight was obtained, and photo physical properties were characterized in DCM.

PPE-1-Br: GPC: $\mathrm{M}_{\mathrm{w}}=13.66 \mathrm{kDa} ; \mathrm{M}_{\mathrm{n}}=09.30 \mathrm{kDa}$; PDI $=1.42$. UV-vis: $\lambda_{\max }=400 \mathrm{~nm}$; fluo $\lambda_{\max }=467$ $\mathrm{nm} ; \mathrm{QY}=69 \%$ and $\varepsilon=42716.79 \mathrm{M}^{-1} \mathrm{~cm}^{-1}$ (Per repeating Unit) in DCM. ${ }^{1} \mathrm{H}$ NMR (400 MHz, $\left.\mathrm{CDCl}_{3}\right): \delta 7.91$ (br, 1H), 7.51 (br, 2H), 7.39 (br, 2H), 6.96 (br, 4H), 4.16 (br, 8H), 3.84 (br, 8H), 3.70 (br, 15H), 3.57 (br, 10H), 3.44 (br, 4H), 3.54 (br, 4H), 3.27 (br, 4H) 3.02 (br, 2H), 2.75 (br, 2H). FT-IR (neat): v = 2924, 2868, $1671,1589,1512,1408,1352,1265,1214,1097,1041,937,838 \mathrm{~cm}^{-1}$. 


\section{Synthesis of PPE-1.}

Triphenyl phosphine (141 mg, $0.540 \mathrm{mmol}$ ) was dissolved in chloroform and the resulting solution was added to a stirred suspension of PPE-1-Br (100 mg, $0.054 \mathrm{mmol}$ ). The reaction was heated to $85{ }^{\circ} \mathrm{C}$ for $12 \mathrm{~h}$. The resulting mixture was purified by precipitation in ethyl acetate (x5). Yield: $70 \mathrm{mg}$ (44.7\%).

PPE-1: UV-vis: $\lambda_{\max }=405 \mathrm{~nm}$; fluo $\lambda_{\max }=472 \mathrm{~nm}$; QY $=40 \%$ and $\varepsilon=67606.65 \mathrm{M}^{-1} \mathrm{~cm}^{-1}$ (Per repeating Unit) in DMSO. ${ }^{1} \mathrm{H}$ NMR (400 MHz, DMSO-d6): $\delta 10.34$ (br, 1H), $7.75(\mathrm{br}, 33 \mathrm{H}), 7.41$ (br, 4H), 7.24 (br, 2H), 7.14 (br, 2H), 4.16 (br, 9H), 3.89 (br, 5H), 3.78 (br, 6H), 3.62 (br, 17H), 3.47 (br, 8H), 3.28 (br, 3H), 3.17 (br, 4H), 2.81 (br, 2H). ${ }^{31} \mathrm{P}$ NMR (161.9 MHz, DMSO-d6): 25.38. FT-IR (neat): $v=2921,2870,1683$, $1597,1520,1430,1408,1210,1099,1047 \mathrm{~cm}^{-1}$.

CPN-1: UV-vis: $\lambda_{\max }=407 \mathrm{~nm}$; Emission $\lambda_{\max }=470 \mathrm{~nm}$; QY $=14 \%$ were measured using $1 \%$ DMSO in water $(\mathrm{v} / \mathrm{v})$.
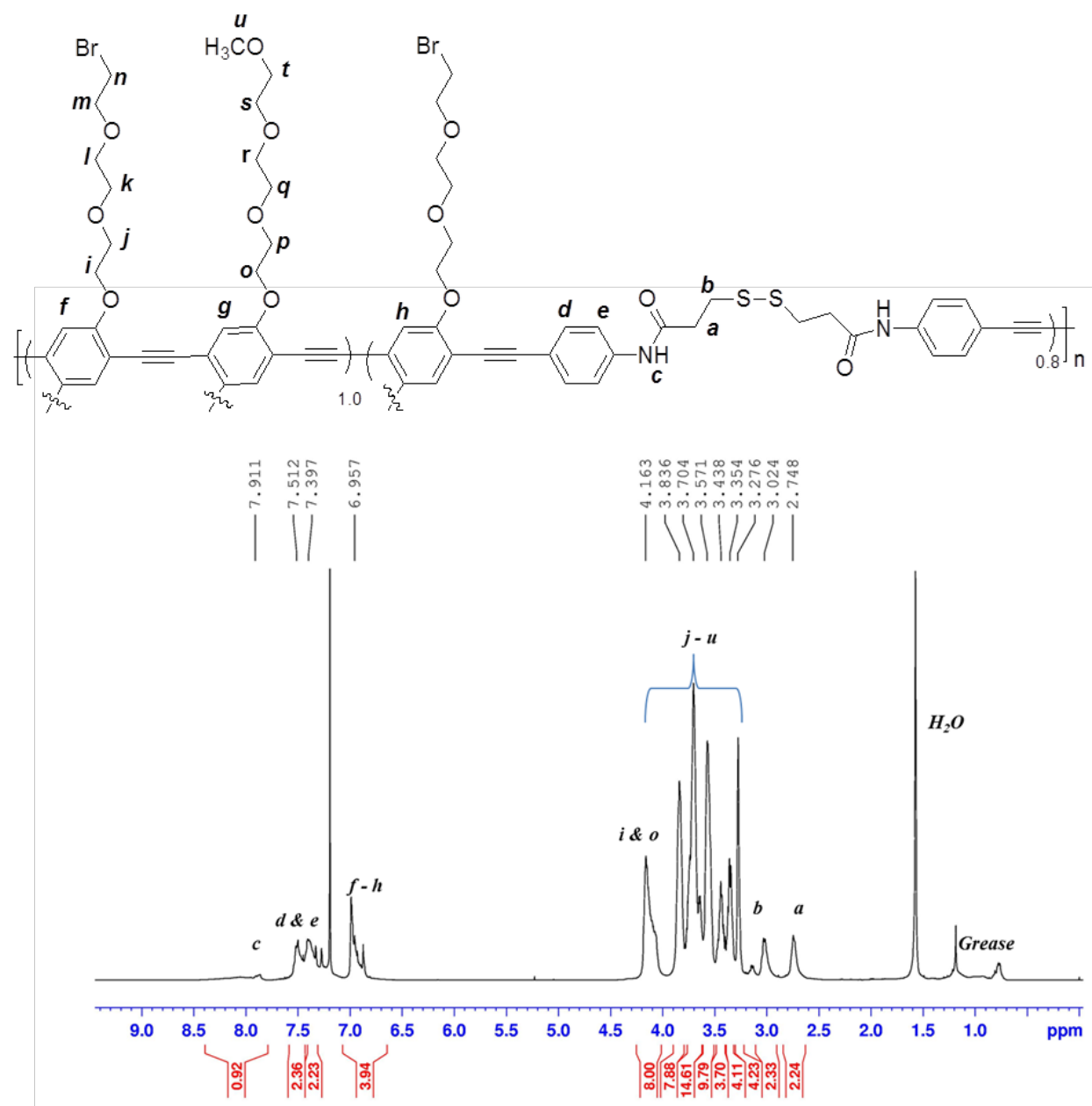

Figure S4.3. ${ }^{1} \mathrm{H}$ NMR of PPE-1-Br in $\mathrm{CDCl}_{3}$. 

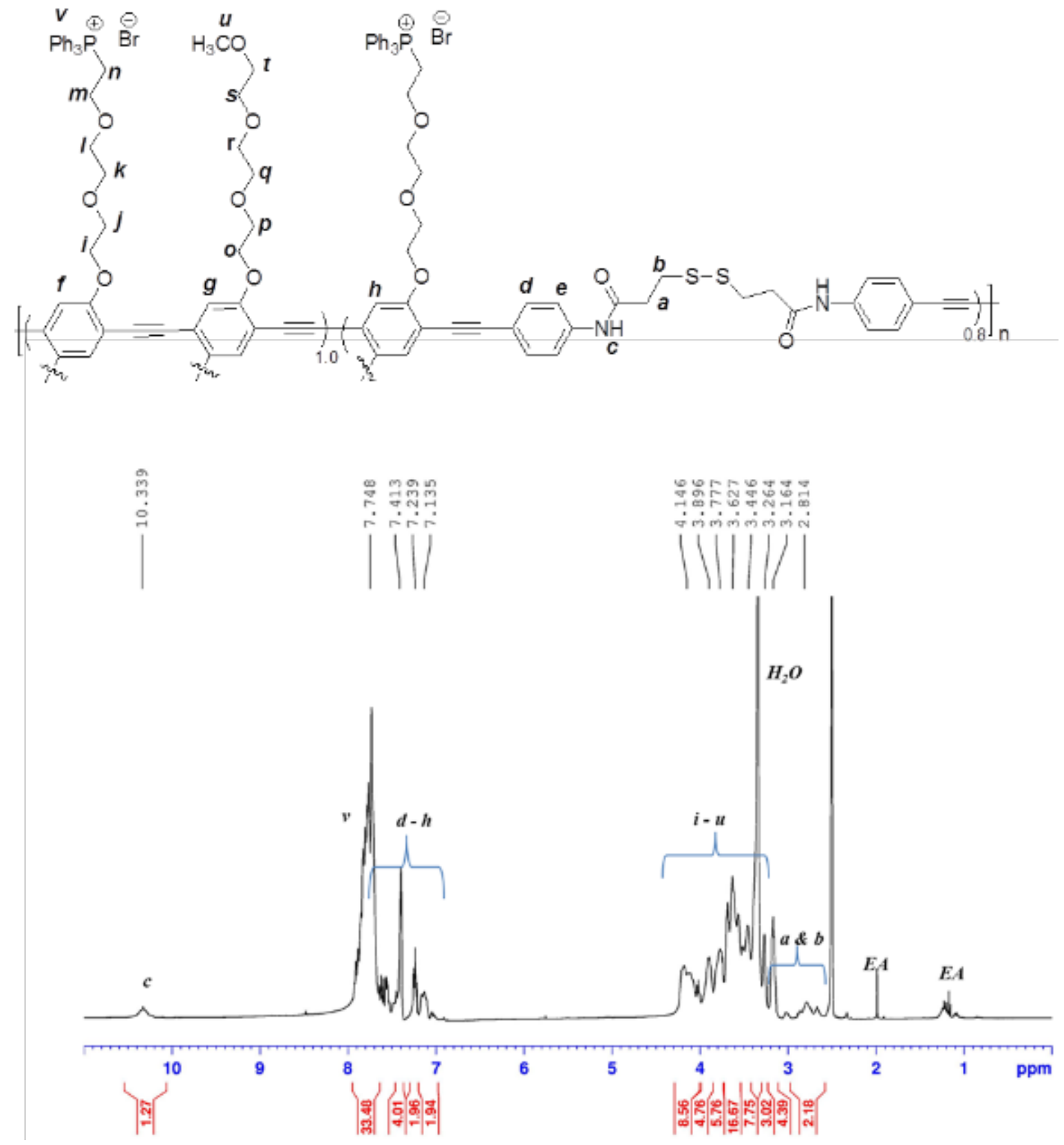

Figure S4.4. ${ }^{1} \mathrm{H}$ NMR of PPE-1 in DMSO-d ${ }_{6}$. 


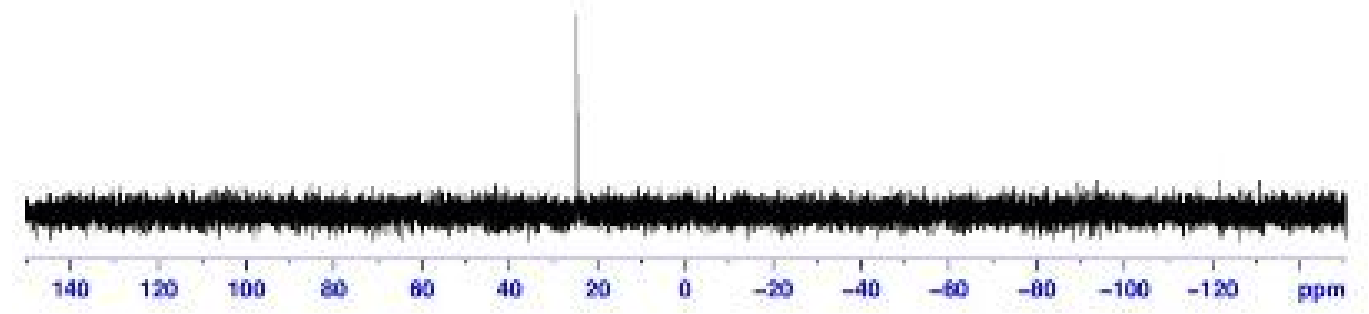

Figure S4.5. ${ }^{31} \mathrm{P}$ NMR of PPE-1 in in DMSO- $\mathrm{d}_{6}$.

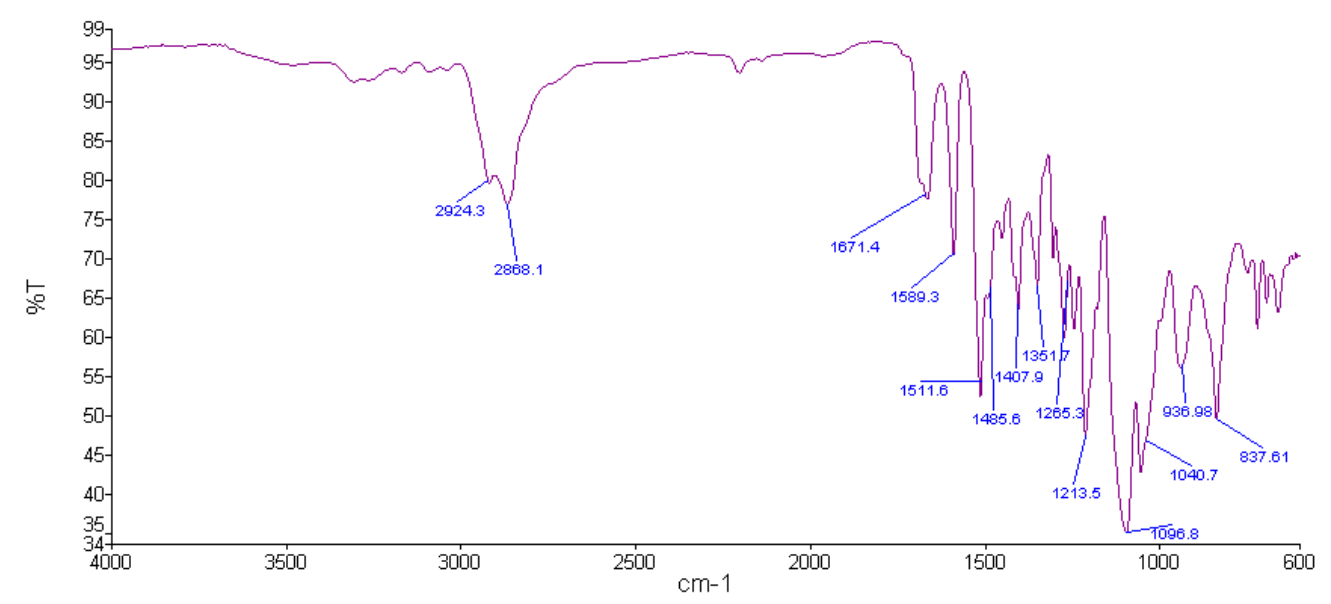

Figure S4.6. FT-IR of PPE-1-Br (neat). 


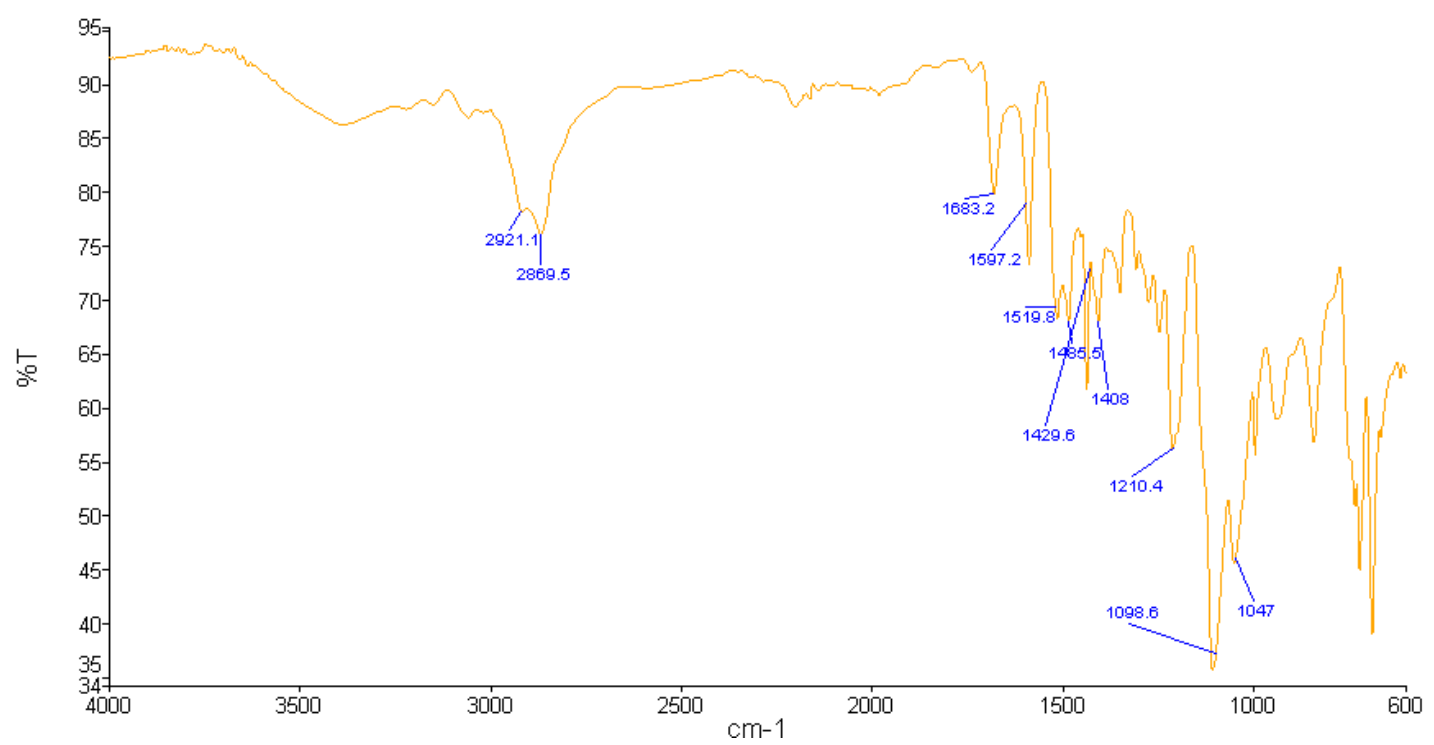

Figure S4.7. FT-IR of PPE-1 (neat).

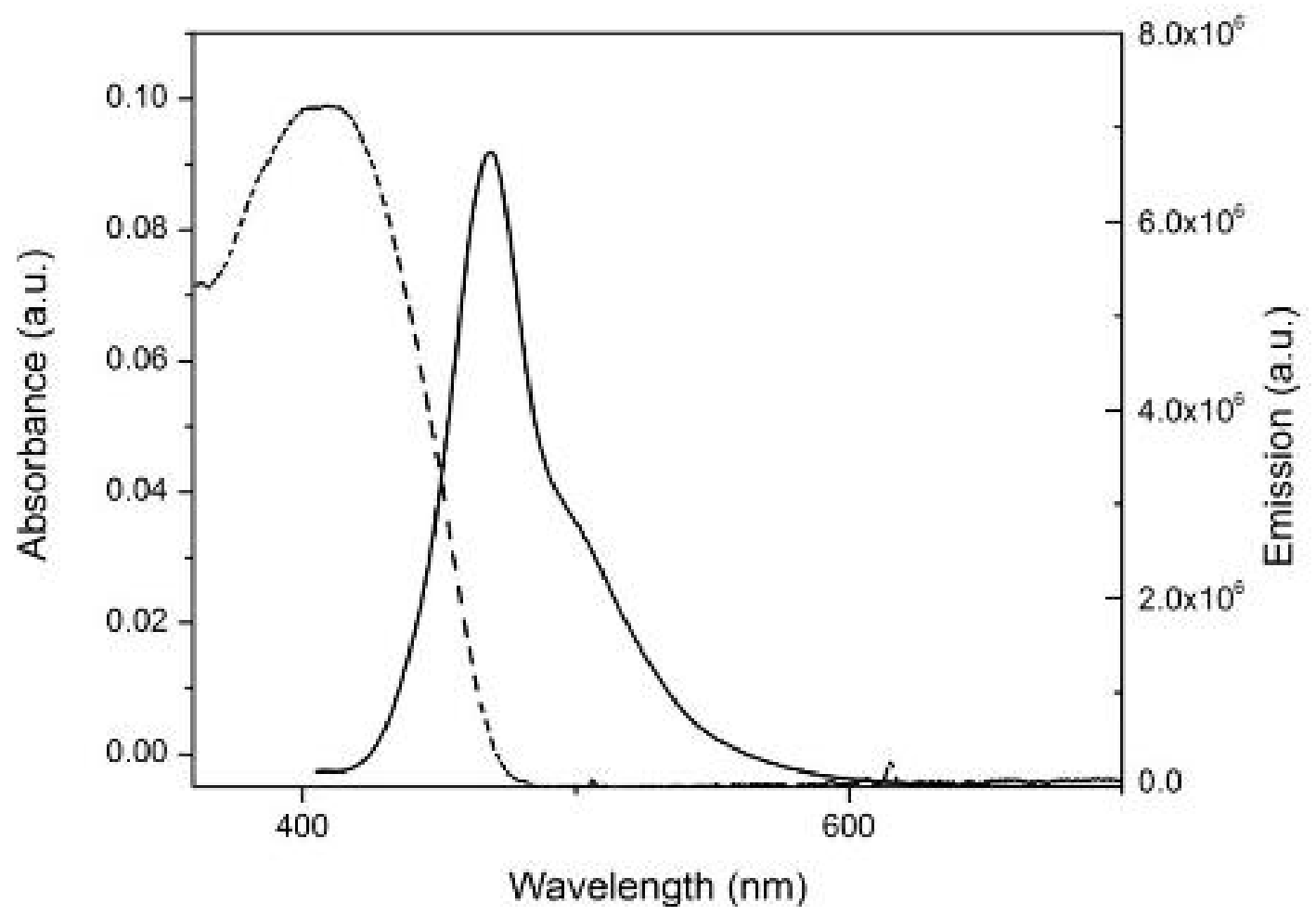

Figure S4.8. UV-vis and emission spectra of PPE-1-Br in DCM. 


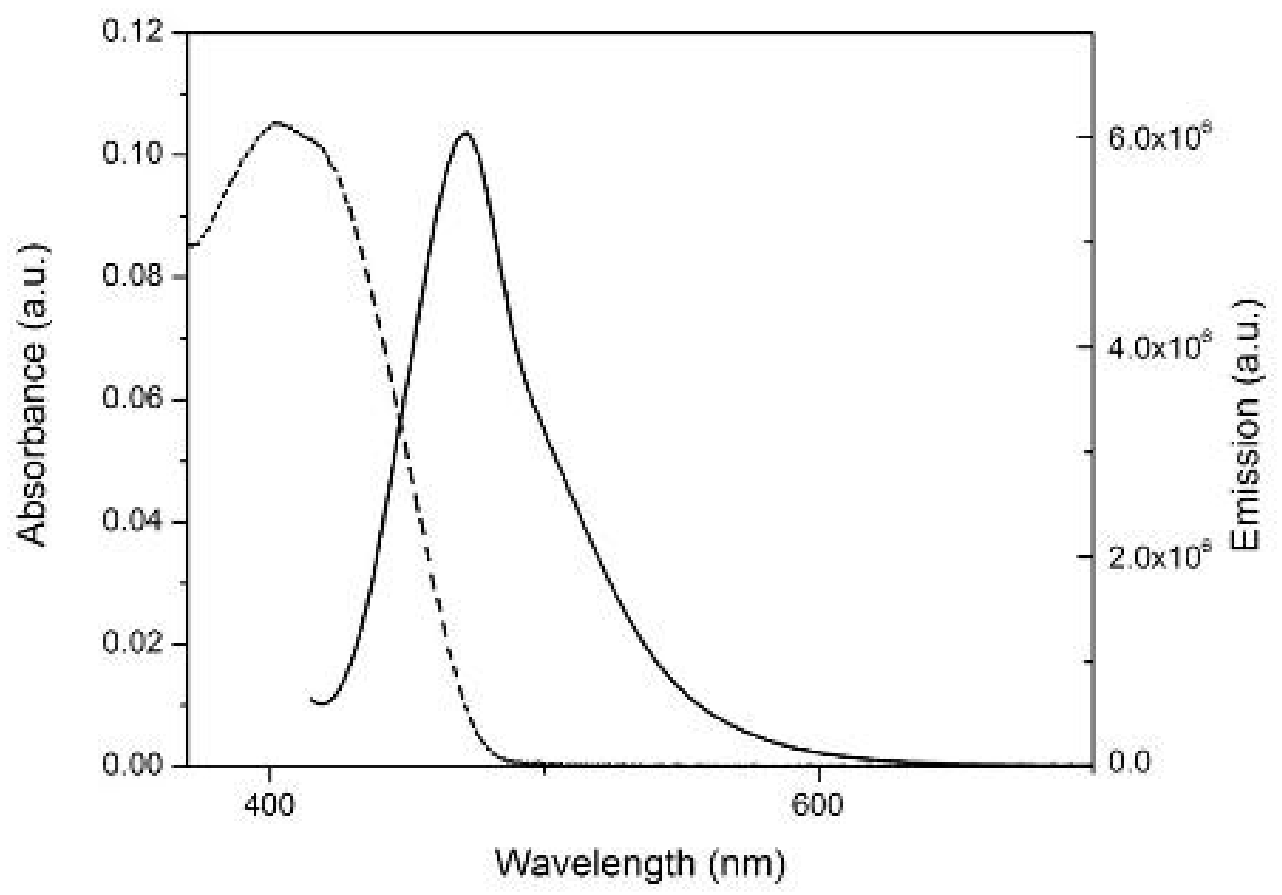

Figure S4.9. UV-vis and emission spectra of PPE-1 in DMSO.

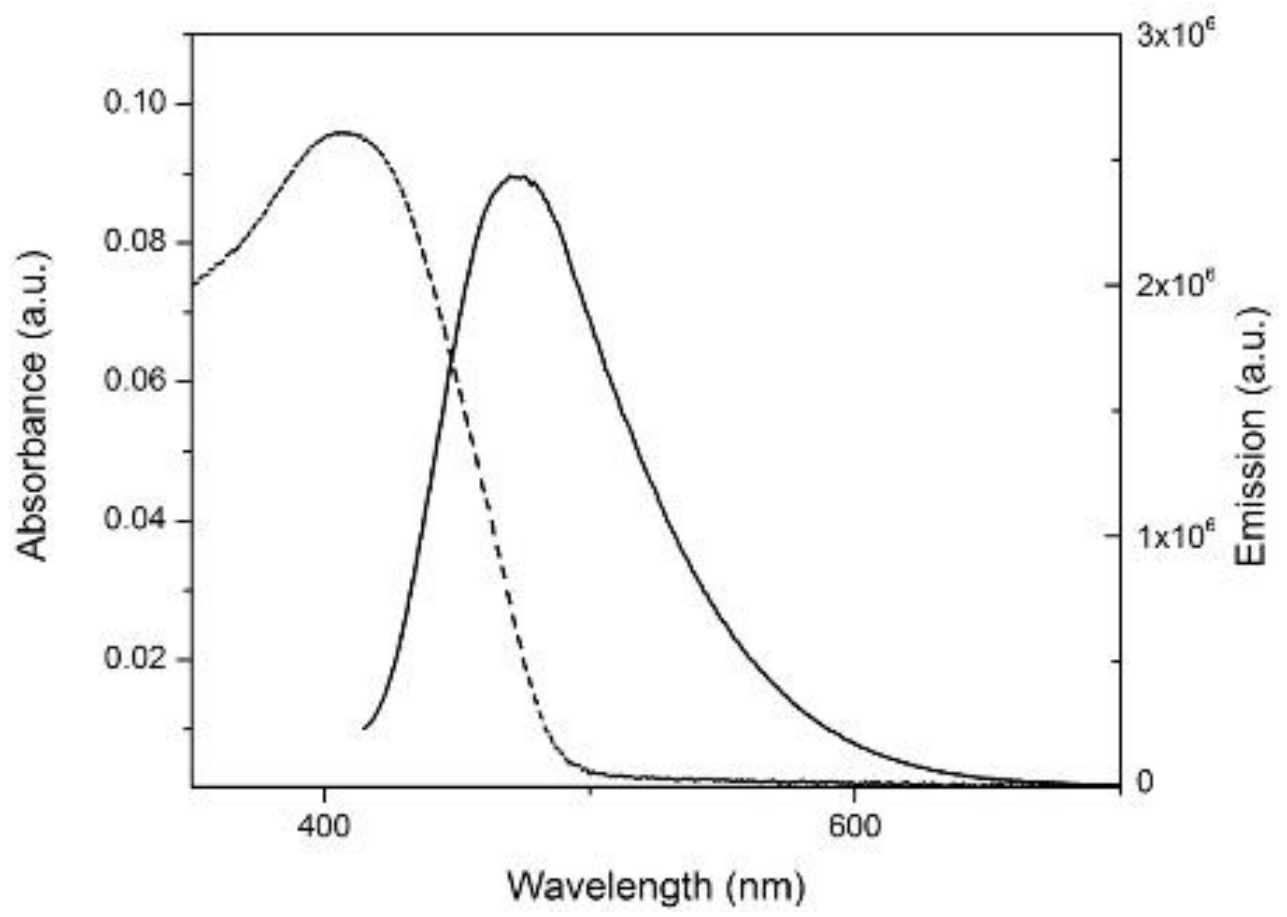

Figure S4.10. UV-vis and emission spectra of CPN-1 1\% DMSO in water (v/v).

\subsubsection{Synthesis of PPE-2.}


Scheme S4.5: Synthetic route to PPE-2
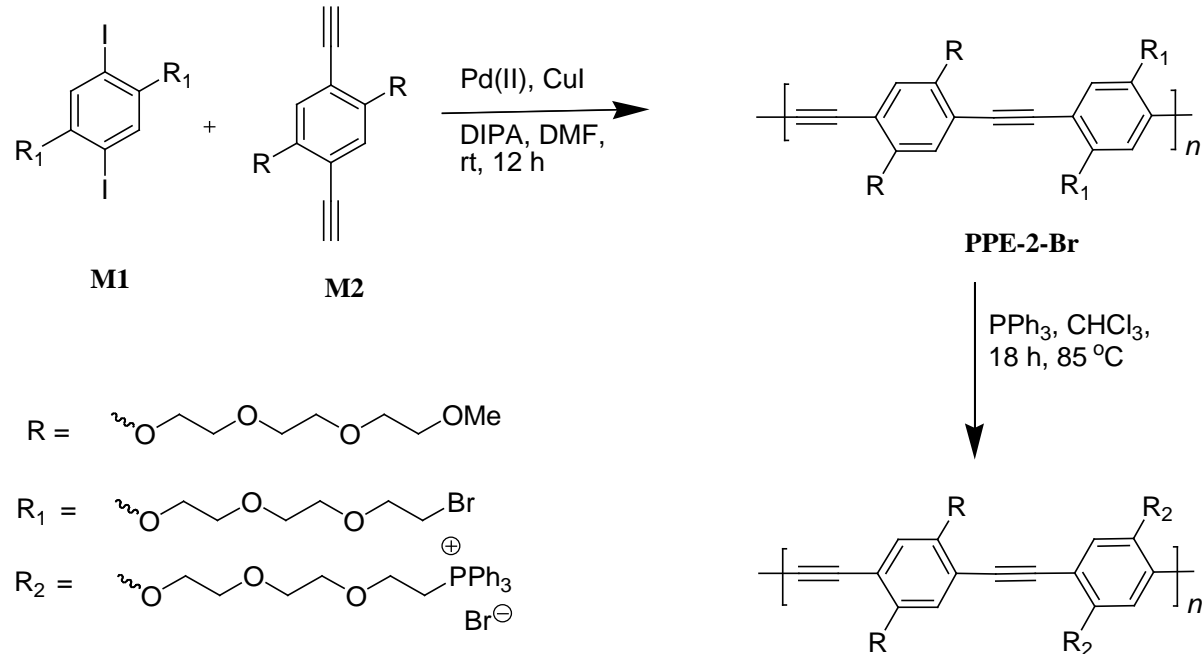

PPE-2-Br

$\mathrm{PPh}_{3}, \mathrm{CHCl}_{3}$ $18 \mathrm{~h}, 85^{\circ} \mathrm{C}$

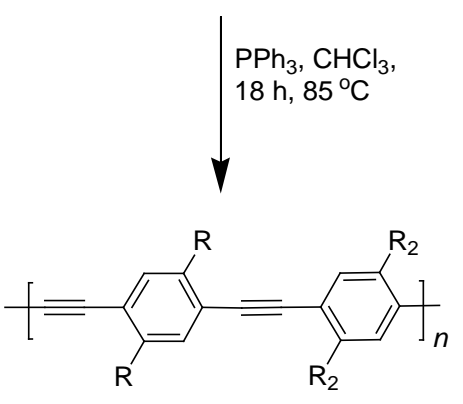

PPE-2

PPE-2-Br. Using the general procedure described above, the polymerization of monomer M1 (100 mg, 0.133 $\mathrm{mmol}$ ) and $\mathbf{M} 2$ (60.0 mg, $0.133 \mathrm{mmol})$ was carried out in presence of $\mathrm{Pd}\left[\left(\mathrm{PPh}_{3}\right)_{2} \mathrm{Cl}_{2}\right](9.3 \mathrm{mg}, 0.013 \mathrm{mmol})$, and $\mathrm{CuI}$ (1.3 mg, $0.007 \mathrm{mmol})$. The resulting mixture was purified by precipitation in methanol (x3) Yield: $0.15 \mathrm{~g}$ (88.5 \%). An aliquot was redissolved in THF, the molecular weight was obtained, and photo physical properties were characterized in DCM.

PPE-2-Br: GPC: $\mathrm{M}_{\mathrm{w}}=26.36 \mathrm{kDa} ; \mathrm{M}_{\mathrm{n}}=15.98 \mathrm{kDa}$; PDI $=1.65 . \mathrm{UV}$-vis: $\lambda_{\max }=432 \mathrm{~nm}$; fluo $\lambda_{\max }=473$ $\mathrm{nm} ; \mathrm{QY}=53 \%$ and $\varepsilon=23526.82 \mathrm{M}^{-1} \mathrm{~cm}^{-1}$ (Per repeating Unit) in DCM. ${ }^{1} \mathrm{H}$ NMR (400 MHz, $\left.\mathrm{CDCl}_{3}\right): \delta 7.06$ (br, 4H), 4.24 (br, 8H), 3.92 (br, 9H), 3.78 (br, 12H), 3.64 (br, 13H), 3.52 (br, 5H), 3.43 (br, 4H), 3.35 (br, 7H). FT-IR (neat): $v=2923,2870,2197,1510,1423,1270,1241,1092,1060 \mathrm{~cm}^{-1}$.

\section{Synthesis of PPE-2.}

Triphenyl phosphine (270 mg, $1.02 \mathrm{mmol}$ ) was dissolved in chloroform and the resulting solution was added to a stirred suspension of PPE-2-Br (100 mg, $0.102 \mathrm{mmol}$ ). The reaction was heated to $85{ }^{\circ} \mathrm{C}$ for $12 \mathrm{~h}$. The resulting mixture was purified by precipitation in ethyl acetate (x5). Yield: 95 mg (61.7\%).

PPE-2: UV-vis: $\lambda_{\max }=436 \mathrm{~nm}$; fluo $\lambda_{\max }=478 \mathrm{~nm}$; $\mathrm{QY}=25 \%$ and $\varepsilon=46128.66 \mathrm{M}^{-1} \mathrm{~cm}^{-1}$ (Per repeating Unit) in DMSO. ${ }^{1} \mathrm{H}$ NMR (400 MHz, DMSO-d6): $\delta 7.75$ (br, 30H), 7.15 (br, 4H), 4.13 (br, 8H), 3.91 (br, 
4H), 3.77 (br, 6H), 3.61 (br, 16H), 3.45 (br, 12H), 3.27 (br, 4.H), 3.15 (br, 10H). ${ }^{31} \mathrm{P}$ NMR (161.9 MHz, DMSO-d6): 24.85. FT-IR (neat): $v=3393,2874,2190,1625,1493,1437,1420,1353,1207,1102$, and 1046 $\mathrm{cm}^{-1}$.

CPN-2: UV-vis: $\lambda_{\max }=431 \mathrm{~nm}$; fluo $\lambda_{\max }=469 \mathrm{~nm} ; \mathrm{QY}=5 \%$ were measured using $1 \%$ DMSO in water $(\mathrm{v} / \mathrm{v})$

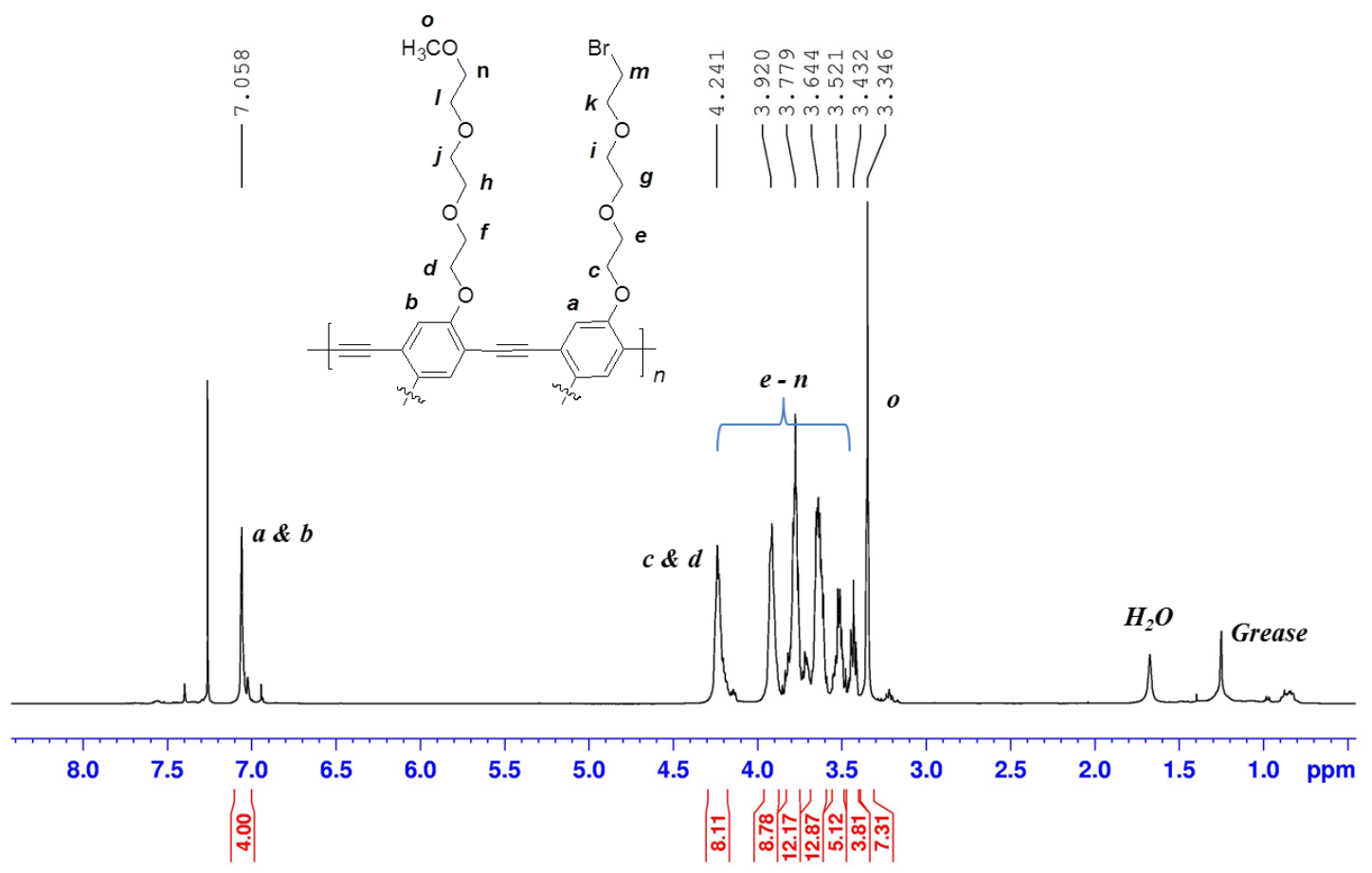

Figure S4.11. ${ }^{1} \mathrm{H}$ NMR of PPE-2-Br in $\mathrm{CDCl}_{3}$. 


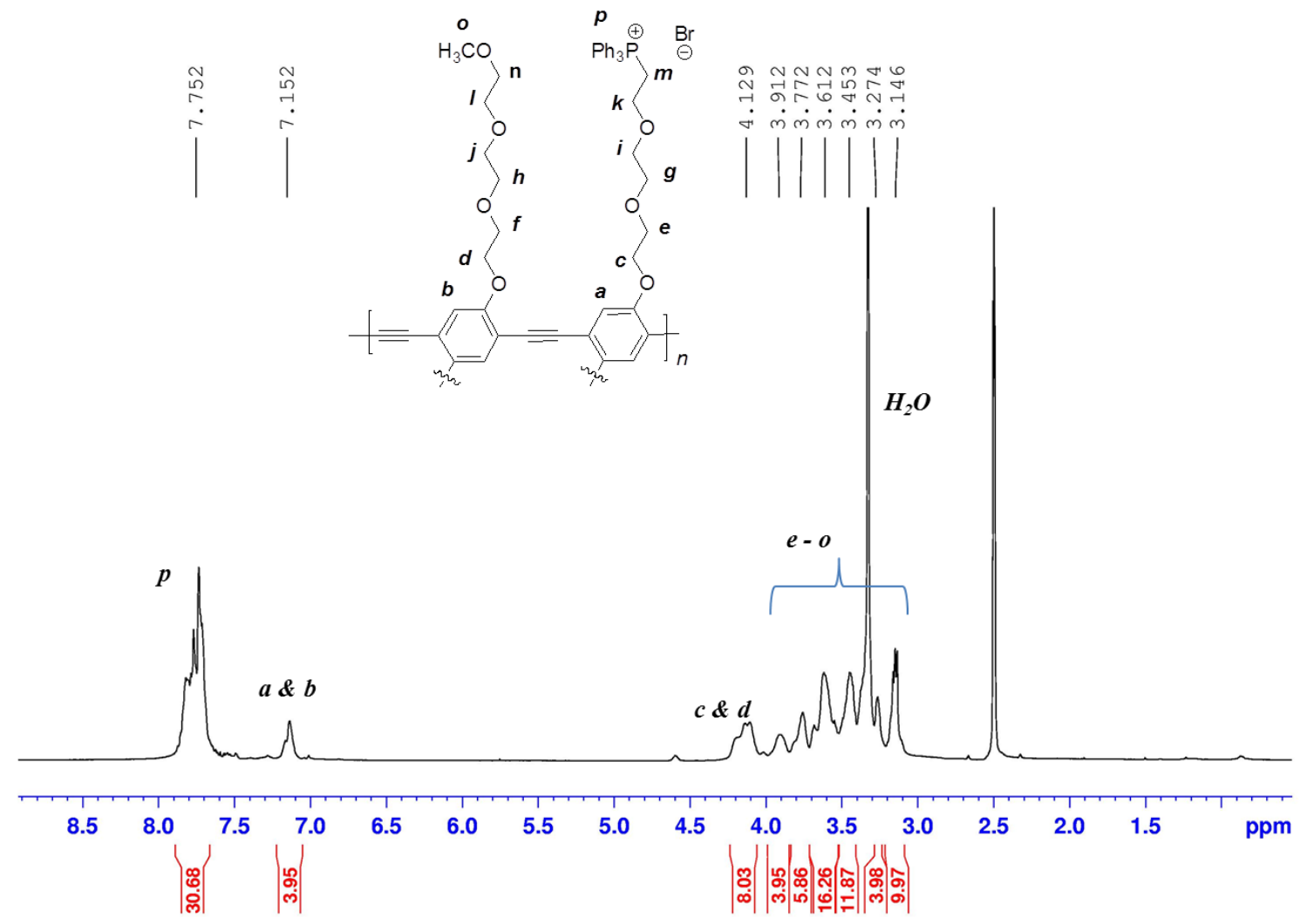

Figure S4.12. ${ }^{1} \mathrm{H}$ NMR of PPE-2 in DMSO- $\mathrm{d}_{6}$.
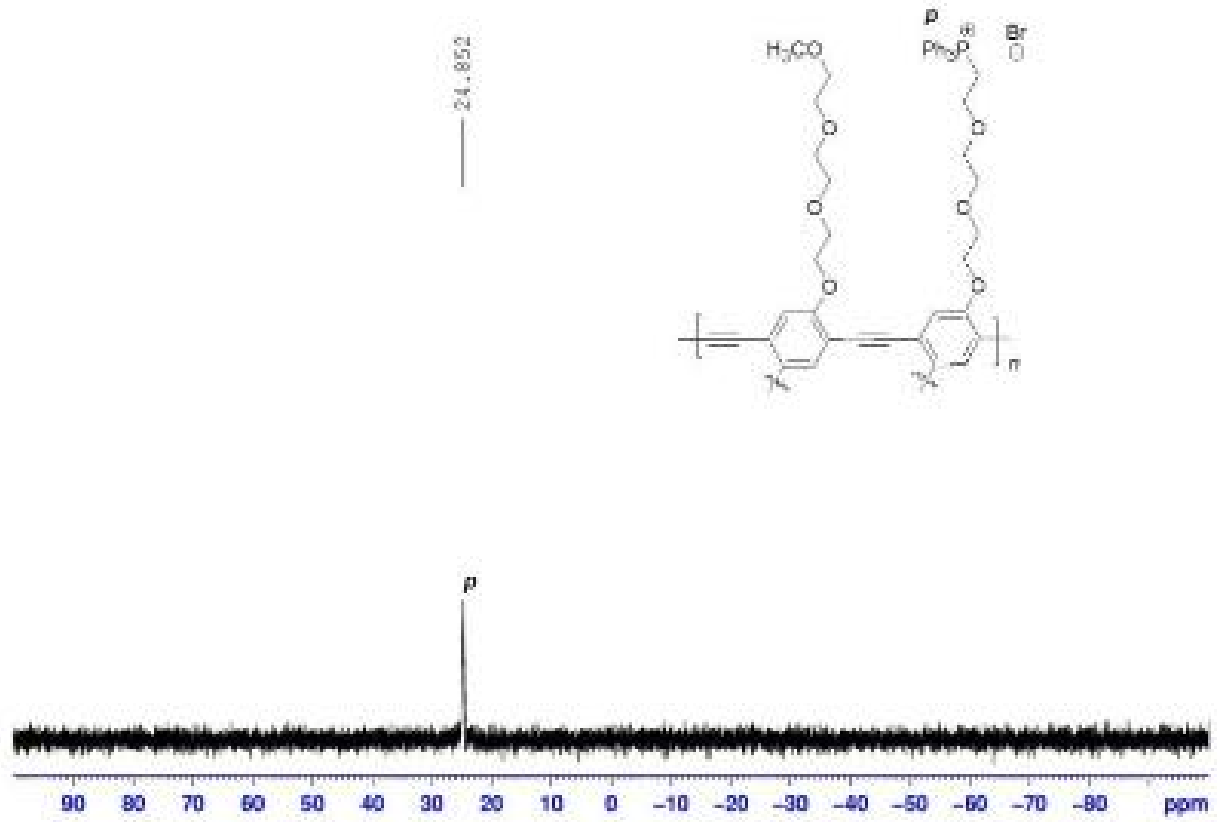

Figure S4.13. ${ }^{31} \mathrm{P}$ NMR of PPE-2 in DMSO- $\mathrm{d}_{6}$. 


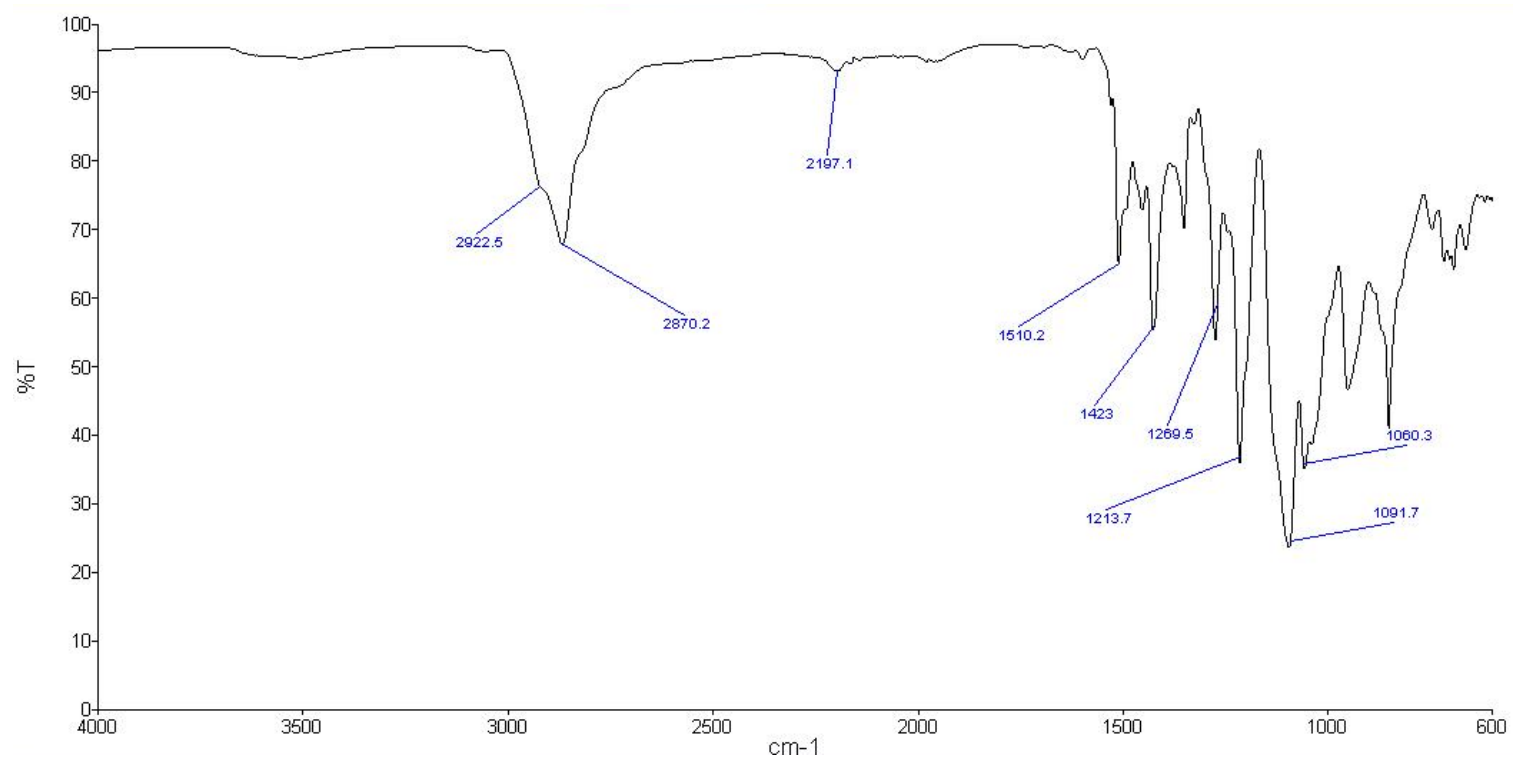

Figure S4.14. FT-IR of PPE-2-Br (neat).

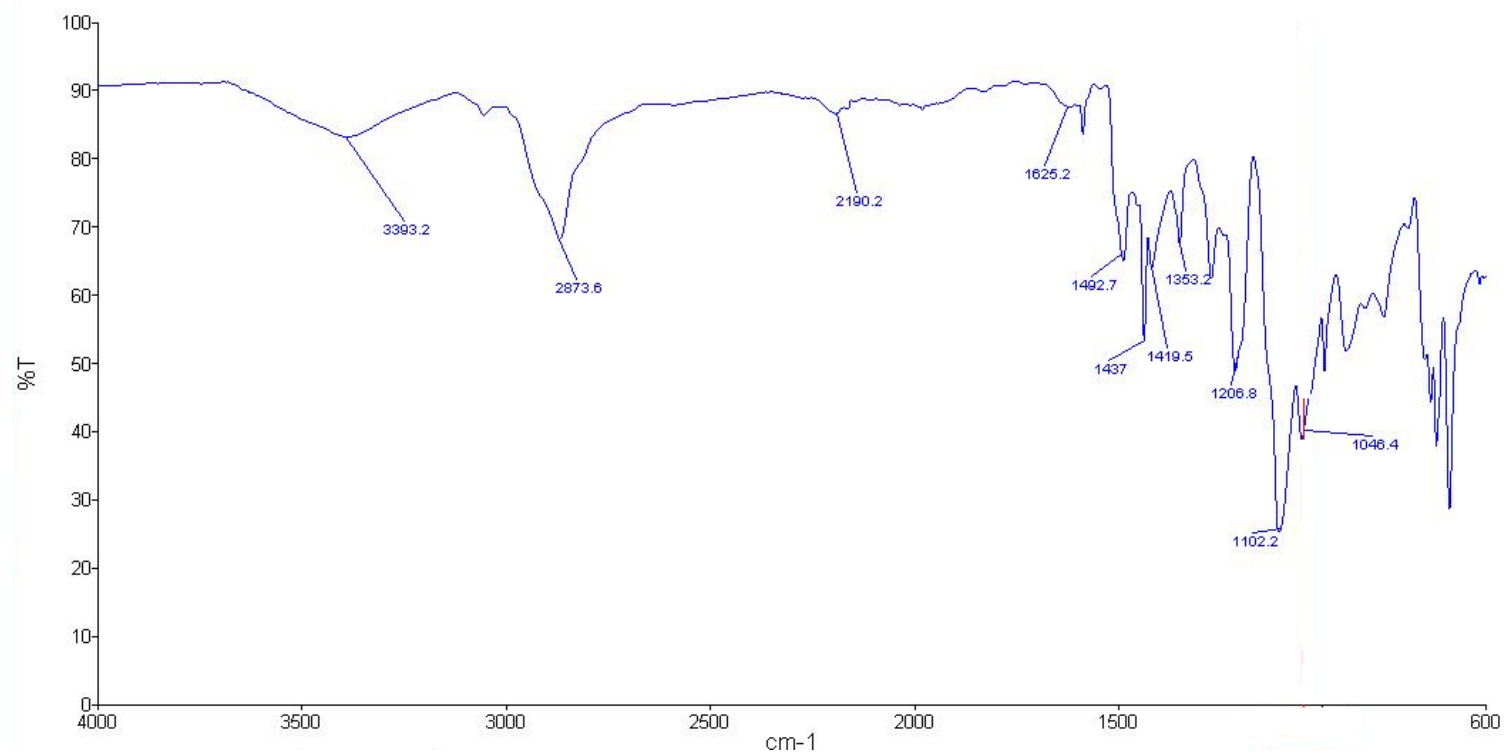

Figure S4.15. FT-IR of PPE-2 (neat). 


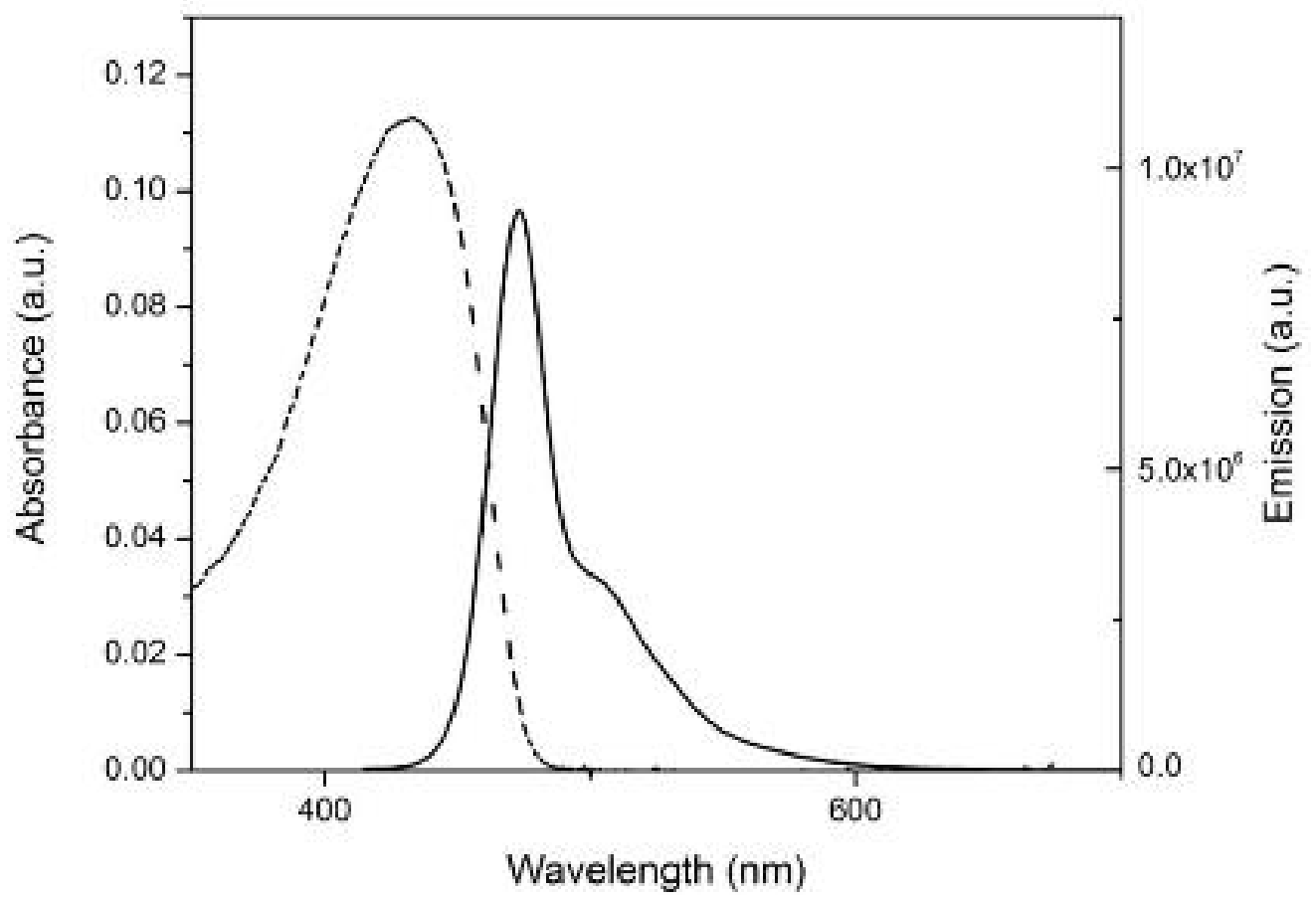

Figure S4.16. UV-vis and emission spectra of PPE-2-Br in DCM.

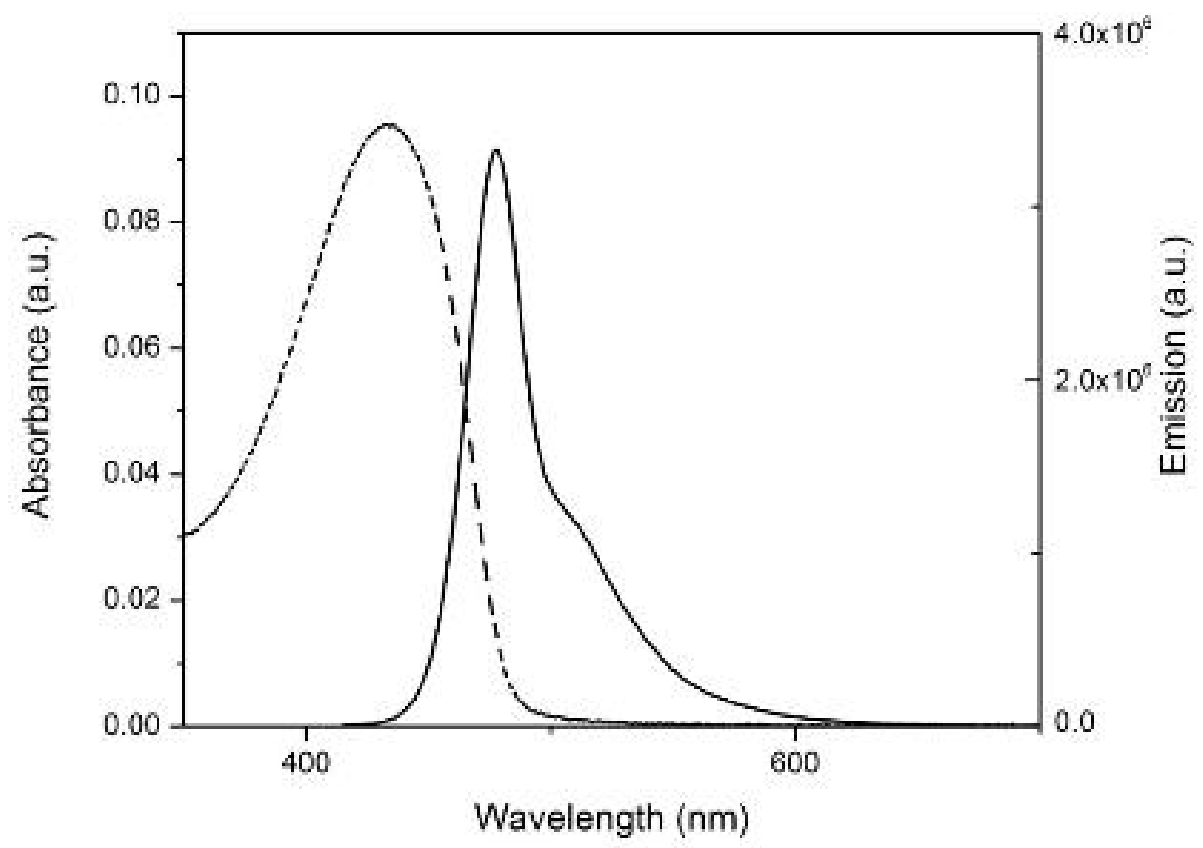

Figure S4.17. UV-vis and emission spectra of PPE-2 in DMSO. 


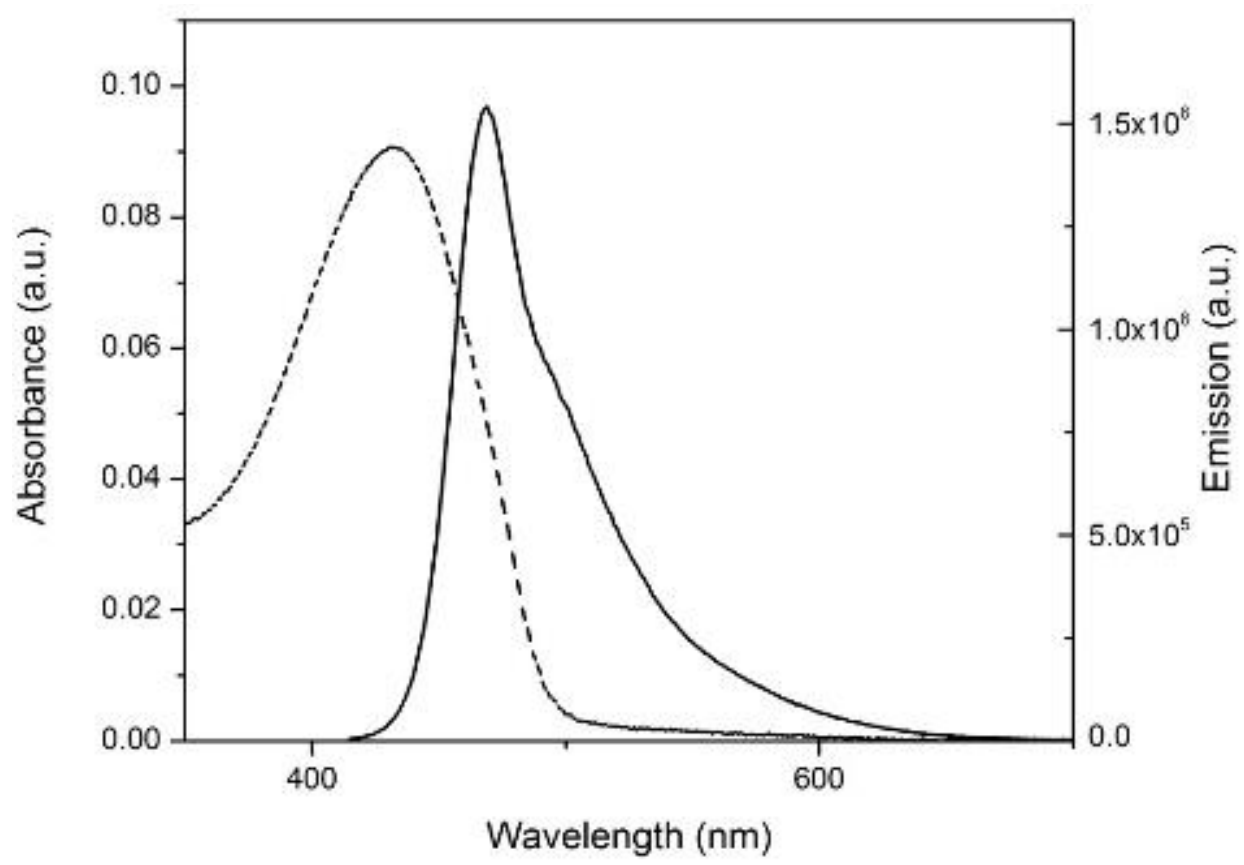

Figure S4.18. UV-vis and emission spectra of CPN-2 1\% DMSO in water (v/v).

\subsubsection{Synthesis of non-degradable PPE with low molecular weight.}

PPE-3-Br. Using general procedure described above, the polymerization of monomer M1 (104.0 mg, 0.138 mmol) and $\mathbf{M} 2$ (50.0 mg, $0.110 \mathrm{mmol})$ in presence of $\mathrm{Pd}\left[\left(\mathrm{PPh}_{3}\right)_{2} \mathrm{Cl}_{2}\right](9.3 \mathrm{mg}, 0.013 \mathrm{mmol})$, and $\mathrm{CuI}(1.3$ mg, 0.007 mmol). Resulting mixture was purified by precipitation in methanol (x3) Yield: 0.09 g (67.6\%). An aliquot was redissolved in THF and molecular weight obtained and photo physical properties characterized in DCM.

PPE-3-Br: GPC: $\mathrm{M}_{\mathrm{w}}=09.62 \mathrm{kDa} ; \mathrm{M}_{\mathrm{n}}=06.96 \mathrm{kDa}$; PDI $=1.38 . \mathrm{UV}$-vis: $\lambda_{\max }=428 \mathrm{~nm}$; fluo $\lambda_{\max }=473$ $\mathrm{nm} ; \mathrm{QY}=15 \%$ and $\varepsilon=15931.37 \mathrm{M}^{-1} \mathrm{~cm}^{-1}$ (Per repeating Unit) in DCM. ${ }^{1} \mathrm{H}$ NMR (400 MHz, $\left.\mathrm{CDCl}_{3}\right): \delta 7.05$ (br, 4H), 4.24 (br, 9H), 3.92 (br, 9H), 3.78 (br, 12H), 3.72 (br, 2H), 3.65 (br, 13H), 3.51 (8H), 3.44 (br, $4 \mathrm{H})$, 3.35 (br, 9H).

\section{Synthesis of PPE-3.}

Triphenyl phosphine (90 mg, $1.02 \mathrm{mmol}$ ) was dissolved in chloroform and resulting solution was added to a stirred suspension of PPE-3-Br (33 mg, $0.102 \mathrm{mmol}$ ). The reaction was heated to $85^{\circ} \mathrm{C}$ for $12 \mathrm{~h}$. The resulting mixture was purified by precipitation in ethyl acetate (x5). Yield: 26 mg (52.0\%). 
PPE-3: UV-vis: $\lambda_{\max }=428 \mathrm{~nm}$; fluo $\lambda_{\max }=476 \mathrm{~nm}$; $\mathrm{QY}=6 \%$ and $\varepsilon=29777.78 \mathrm{M}^{-1} \mathrm{~cm}^{-1}$ (Per repeating Unit) in DMSO. ${ }^{1} \mathrm{H}$ NMR (400 MHz, DMSO-d6): $\delta 7.76$ (br, 30H), 7.14 (br, 4H), 4.16 (br, 8H), 3.91 (br, 4H), 3.76 (br, 6H), 3.61 (br, 14H), 3.45 (br, 12H), 3.26 (br, 4H), 3.14 (br, 9H). ${ }^{31}$ P NMR (161.9 MHz, DMSOd6): 24.84.

CPN-3: UV-vis: $\lambda_{\max }=427 \mathrm{~nm}$; fluo $\lambda_{\max }=468 \mathrm{~nm}$; QY $=5 \%$ were measured using $1 \%$ DMSO in water $(\mathrm{v} / \mathrm{v})$.

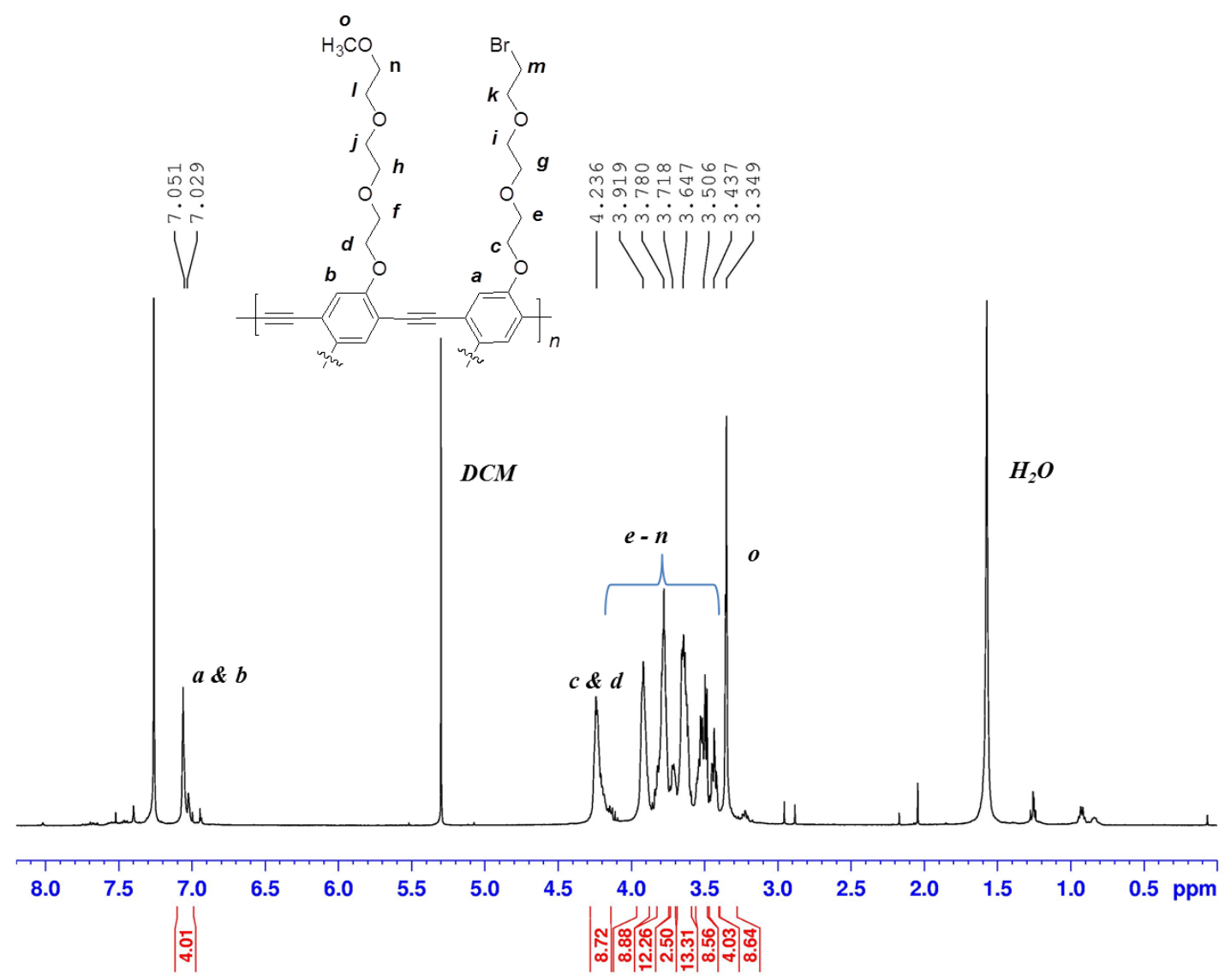

Figure S4.19. ${ }^{1} \mathrm{H}$ NMR of PPE-3-Br in $\mathrm{CDCl}_{3}$. 


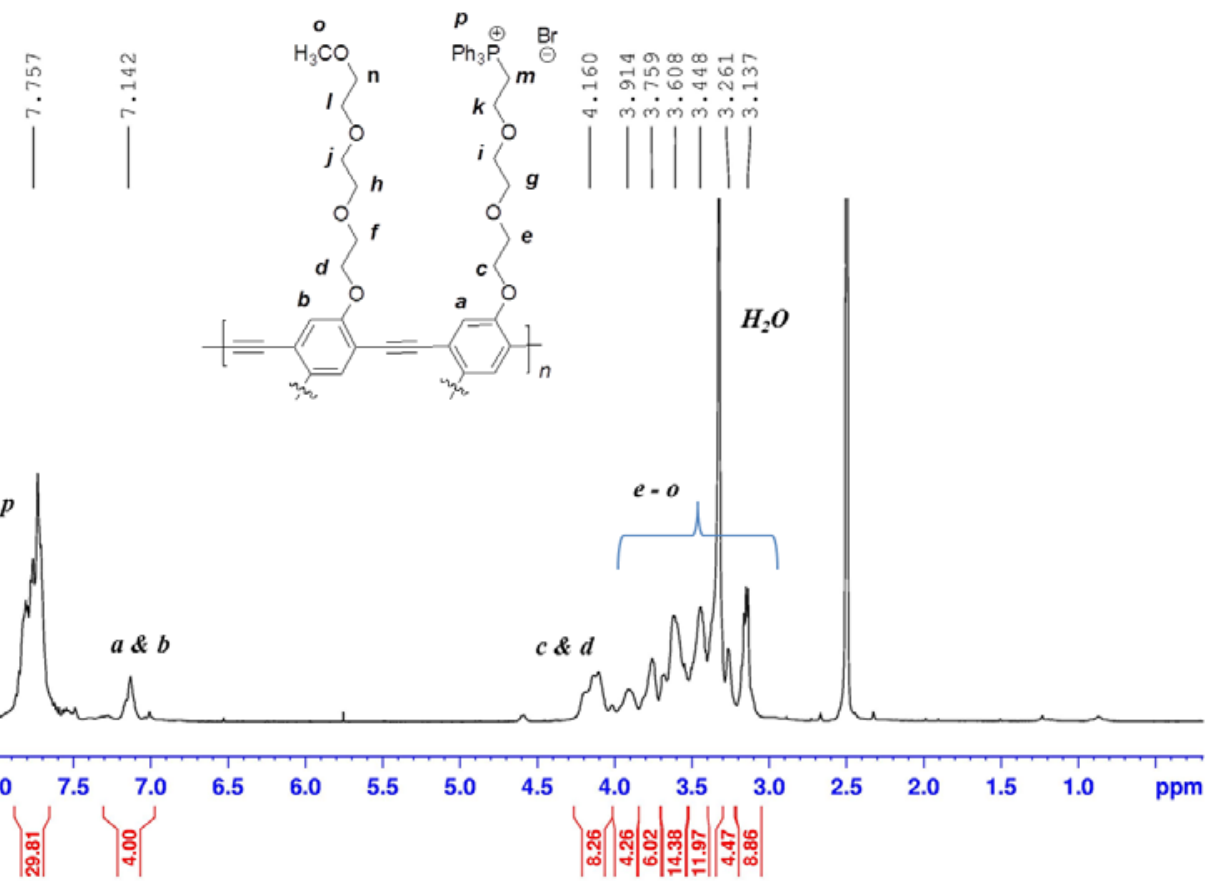

Figure S4.20. ${ }^{1} \mathrm{H}$ NMR of PPE-3 in DMSO- $\mathrm{d}_{6}$.

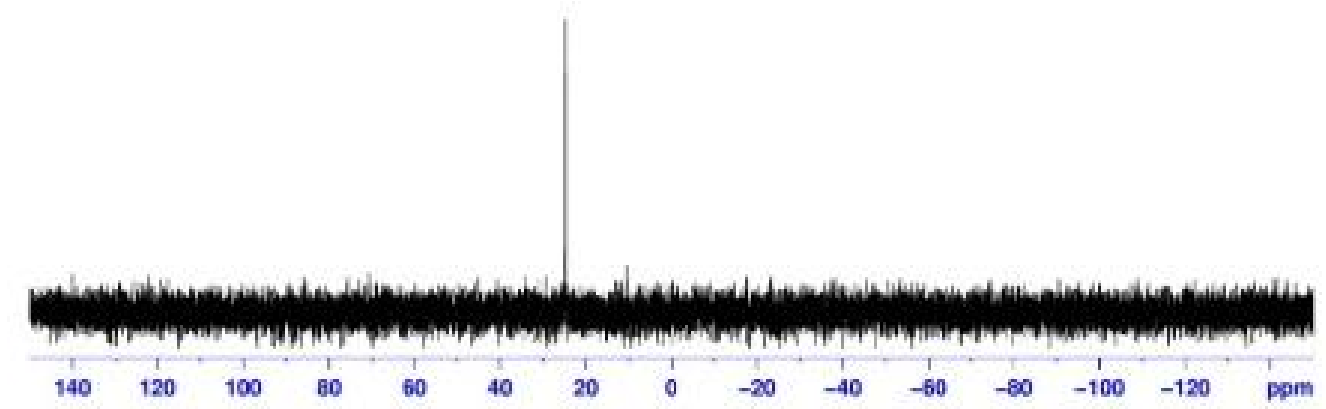

Figure S4.21. ${ }^{31} \mathrm{P}$ NMR of PPE-3 in DMSO- $\mathrm{d}_{6}$. 


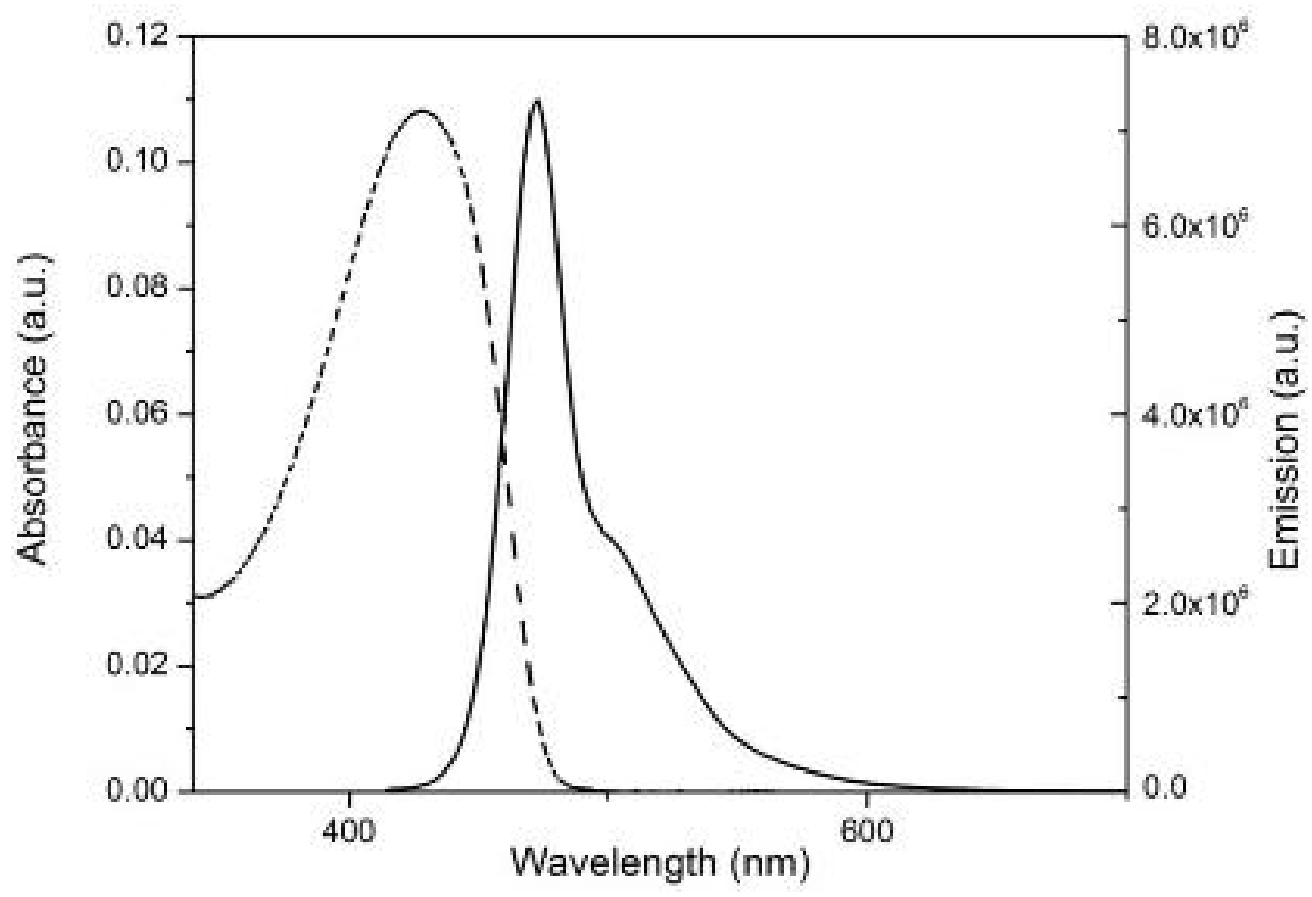

Figure S4.22. UV-vis and emission spectra of PPE-3-Br in DCM.

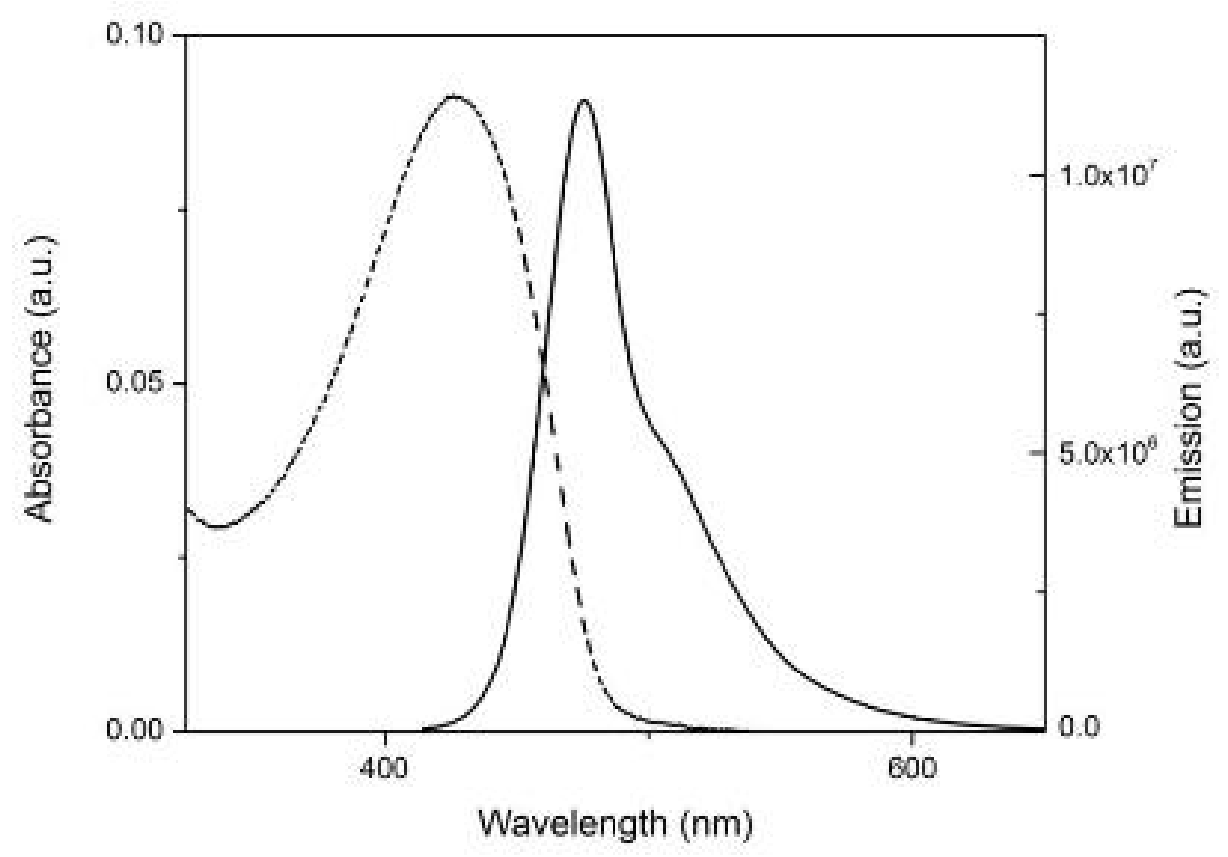

Figure S4.23. UV-vis and emission spectra of PPE-3 in DMSO. 


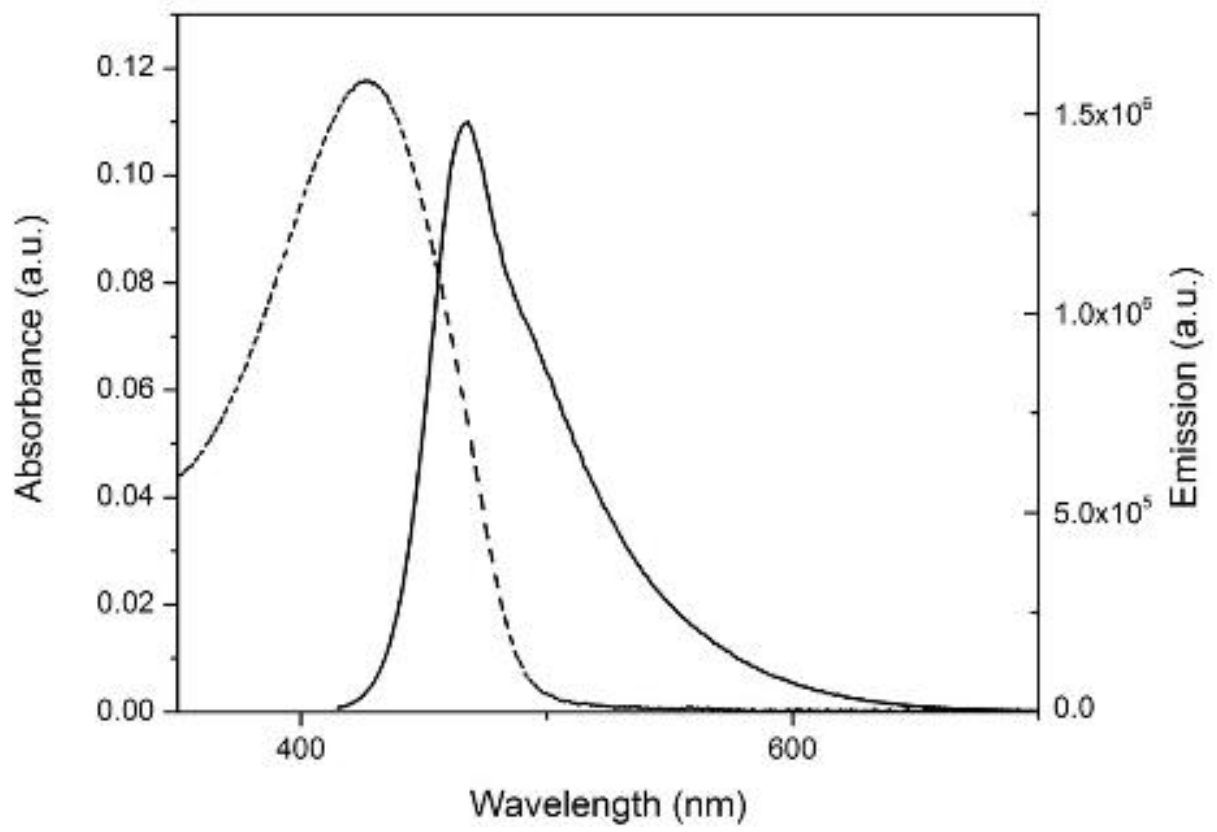

Figure S4.24. UV-vis and emission spectra of CPN-3 1\% DMSO in water (v/v).

\subsubsection{Particle formation and HA complexation.}

Particles were prepared prior to each experiment from a concentrated polymer stock solution in DMSO by diluting with an appropriate volume of filtered (0.22 $\mu \mathrm{m}$ PTFE syringe filter) deionized water $(18 \Omega)$. Sodium hyaluronate (HA) was purchased from Lifecore (MW $40 \mathrm{KDa}$ ) and used as received. A stock solution was prepared by dissolving $2.0 \mathrm{mg}$ of $\mathrm{HA}$ in $1 \mathrm{~mL}$ of deionized water. CPN/HA complexes were prepared by mixing CPN and HA at a molar ratio of 1 to 3 (based on polymer and disaccharide repeating unit) then diluting to the desired concentration with deionized water. CPN/HA complexes were allowed to incubate for 30 minutes prior to measurement.

\subsubsection{Determination of hydrodynamic diameters of CPNs and CPN/HA complexes.}

Nanoparticle tracking analysis (NTA) measurements were performed with a LM10 HS (NanoSight, Amesbury, United Kingdom), equipped with a sCMOS camera, sample chamber with a $488 \mathrm{~nm}$ blue laser, and Viton fluoroelastomer o-ring. The CPN samples were prepared from concentrated stock solutions of polymer in DMSO, at approximately $10 \mu \mathrm{M}$ in a $0.5 \%$ DMSO (v/v) solution using deionized water (18 $\Omega$ ) filtered through $0.22 \mu \mathrm{m}$ polytetrafluoroethylene (PTFE) syringe filter. The samples were injected into the sample chamber with $1 \mathrm{~mL}$ sterile syringes (Restek Corporation, Pennsylvania, USA) until the liquid 
reached the tip of the nozzle. All measurements were performed at $25^{\circ} \mathrm{C}$ using a LM14C temperature controller (NanoSight, Amesbury, United Kingdom). Each sample was measured three times.

Table S4.1. Hydrodynamic diameters and zeta potential of CPNs.

\begin{tabular}{|c|c|c|}
\hline Entry & $\begin{array}{c}\text { Hydrodynamic } \\
\text { diameter (nm) }\end{array}$ & $\begin{array}{c}\text { Zeta potential } \\
(\mathbf{m V})\end{array}$ \\
\hline $\mathrm{CPN}-1$ & $139.9 \pm 3.0$ & $+23.5 \pm 1.84$ \\
\hline $\mathrm{CPN}-1 / \mathrm{HA}$ & $191.8 \pm 2.6$ & $-44.4 \pm 1.01$ \\
\hline $\mathrm{CPN}-2$ & $152.7 \pm 6.7$ & $+29.2 \pm 3.56$ \\
\hline $\mathrm{CPN}-2 / \mathrm{HA}$ & $223.1 \pm 6.9$ & $-42.7 \pm 0.83$ \\
\hline $\mathrm{CPN}-3$ & $158.8 \pm 16.4$ & $+20.1 \pm 5.87$ \\
\hline
\end{tabular}

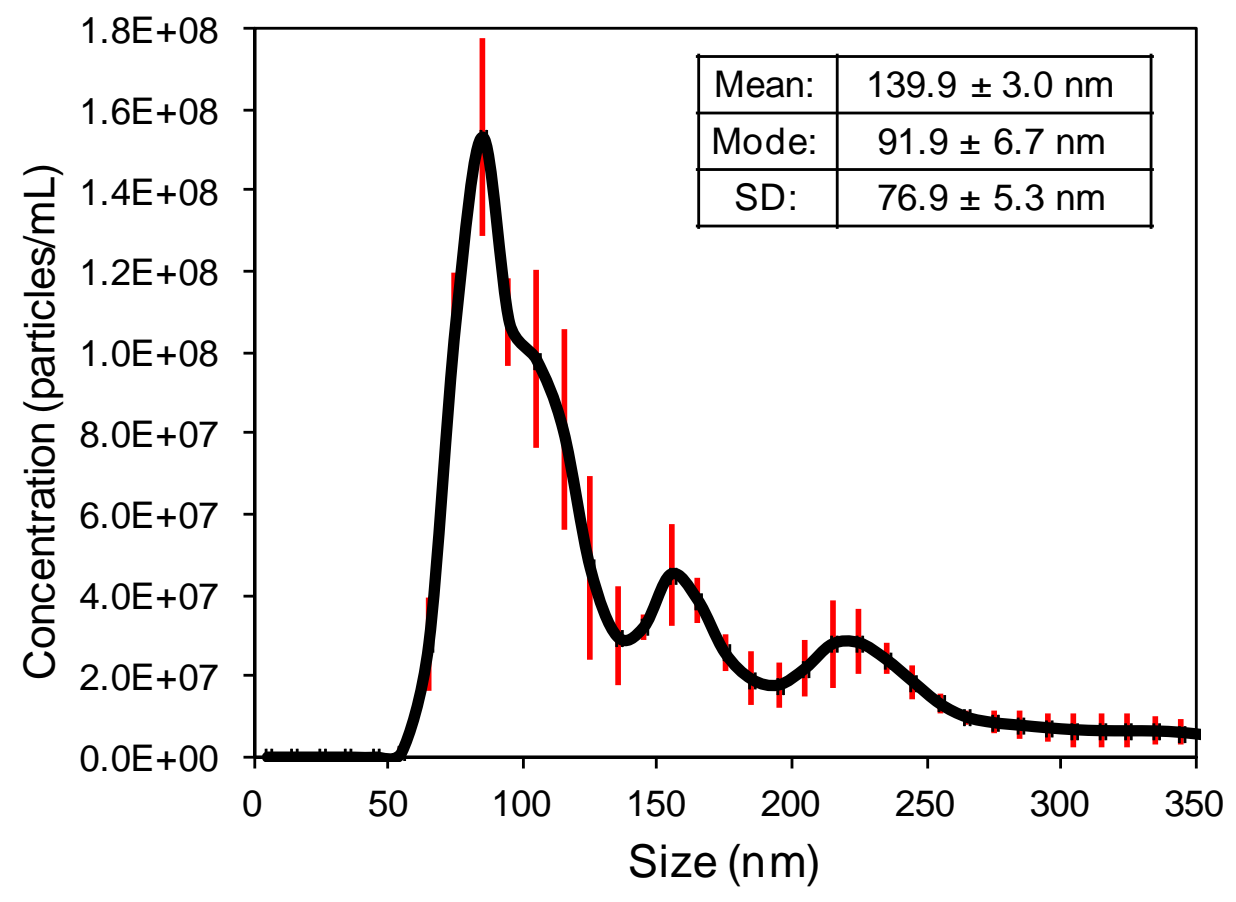

Figure S4.25. NTA for CPN-1. 


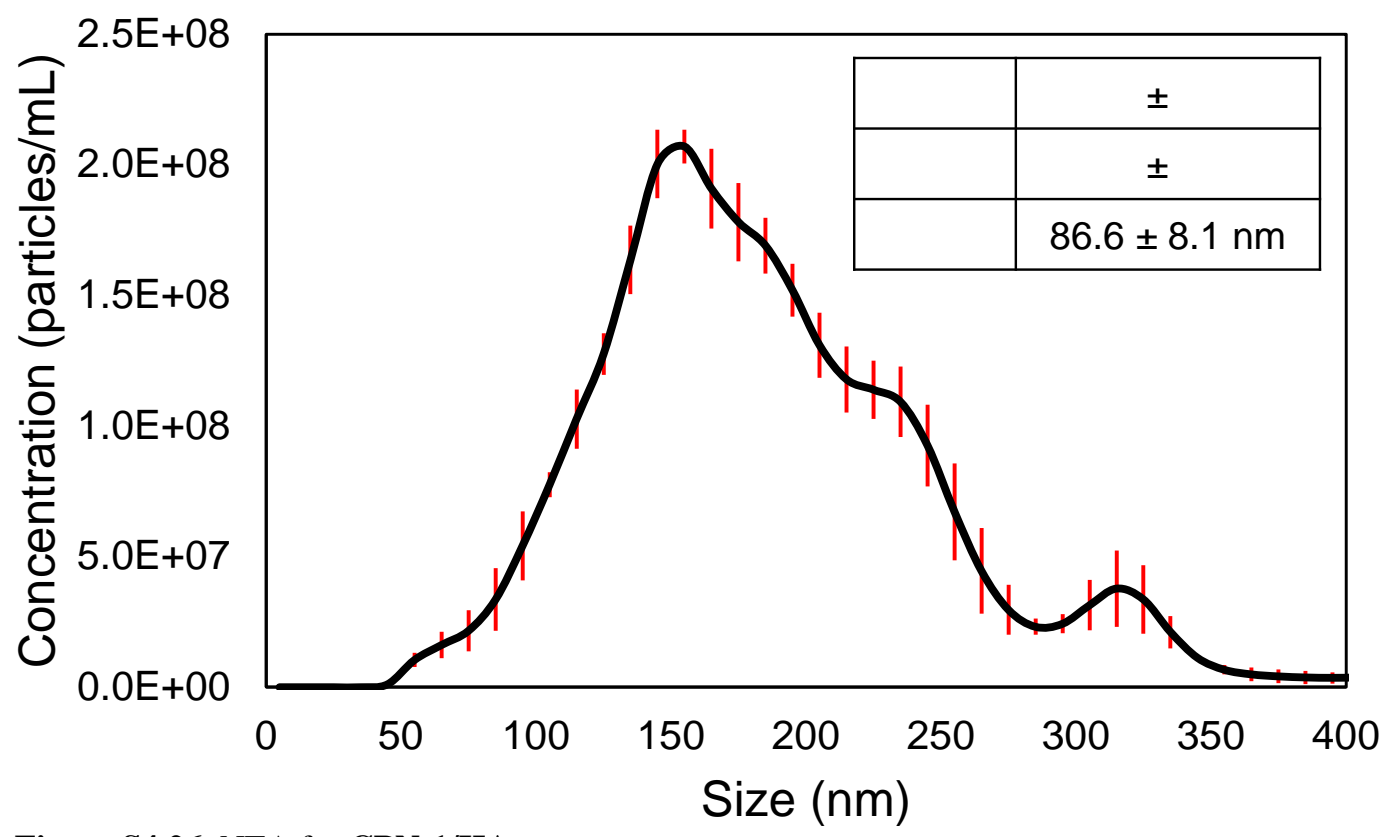

Figure S4.26. NTA for CPN-1/HA.

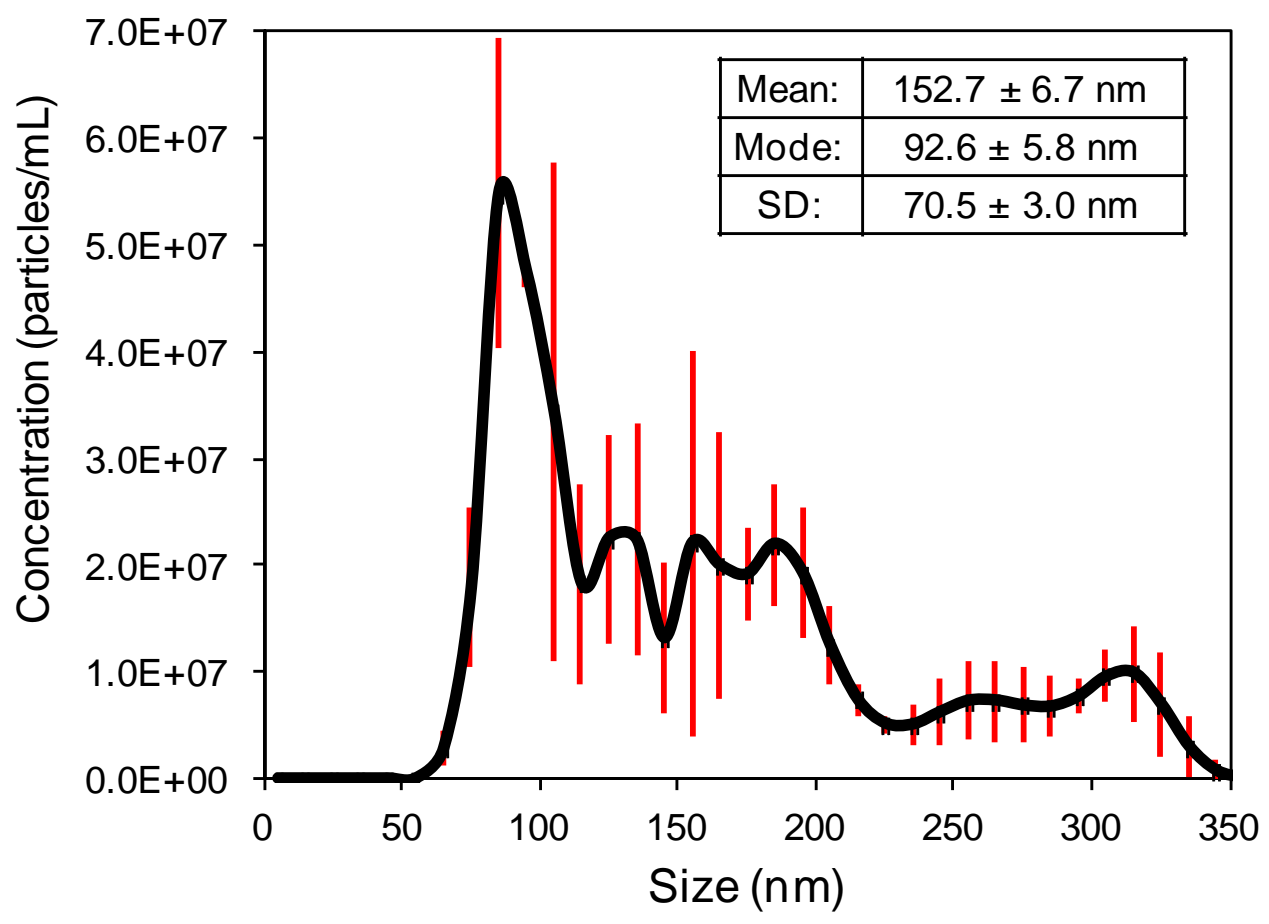

Figure S4.27. NTA for CPN-2. 


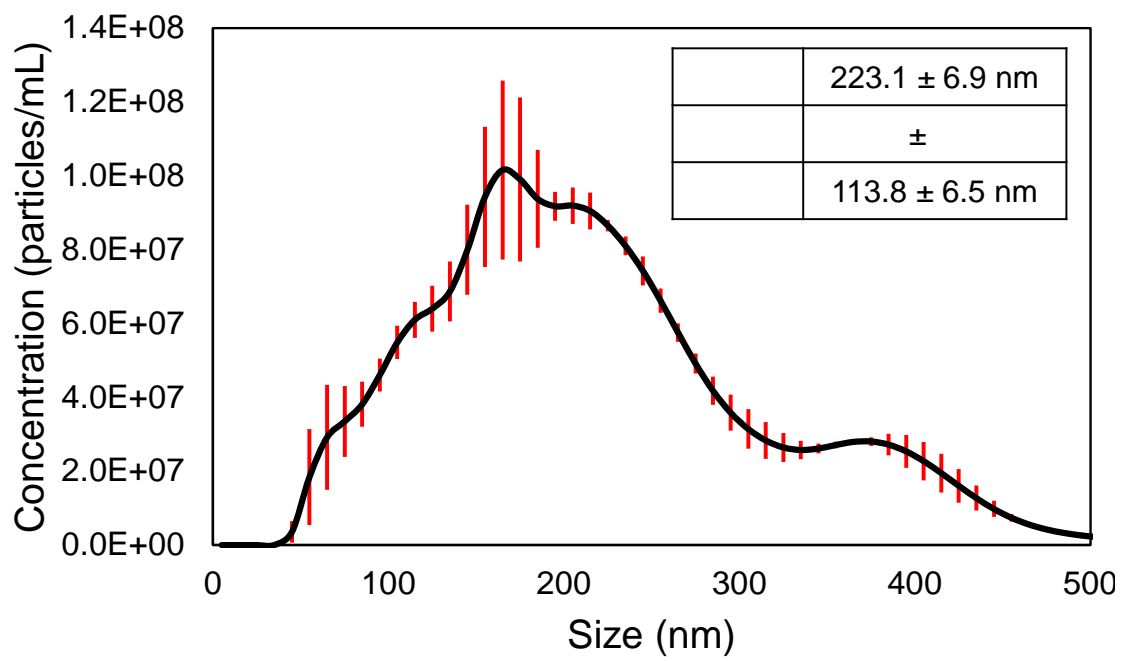

Figure S4.28 NTA for CPN-2/HA.

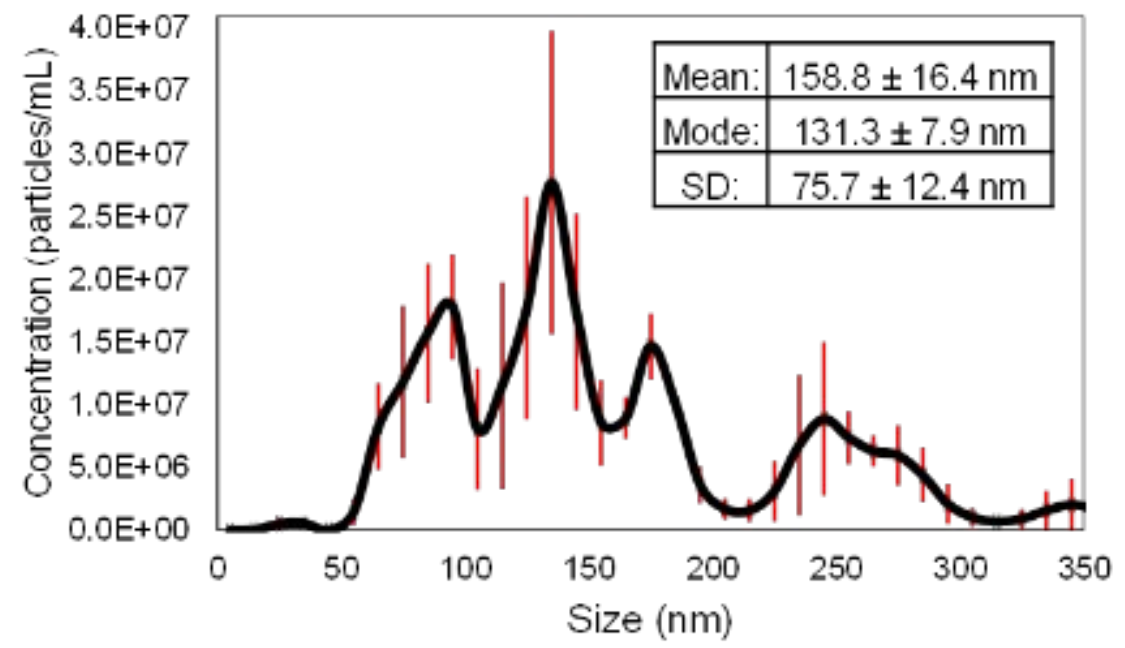

Figure S4.29. NTA for CPN-3.

\subsubsection{Determination of zeta potentials of CPNs and CPN/HA complexes}

Dynamic light scattering (DLS) measurements were performed by Zetasizer nano-ZS (Zen 3600, Malvern Instruments Ltd.) using a folded capillary cell (Catalog \# DTS1060), at room temperature. The CPN samples were prepared from concentrated stock solutions of polymer dissolved in DMSO, at approximately $0.1 \mathrm{mM}$ in a $5 \%$ DMSO $(\mathrm{v} / \mathrm{v})$ solution using deionized water $(18 \Omega)$ filtered through $0.22 \mu \mathrm{m}$ PTFE syringe filter. Each sample was measured six times. 


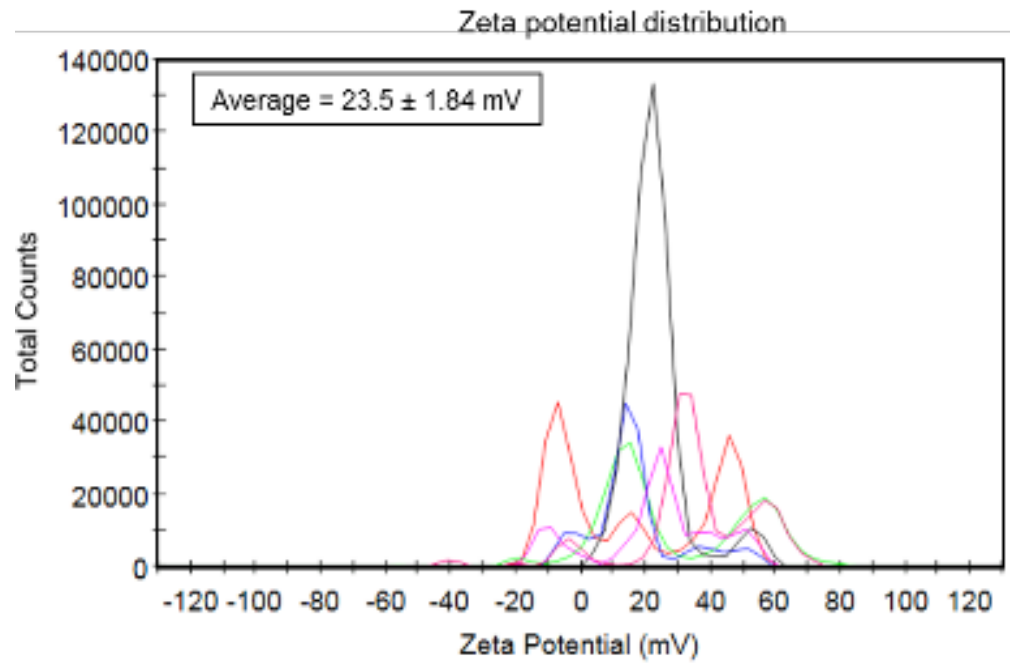

Figure S4.30. Zeta potential of CPN-1.

Zeta Potential Distribution

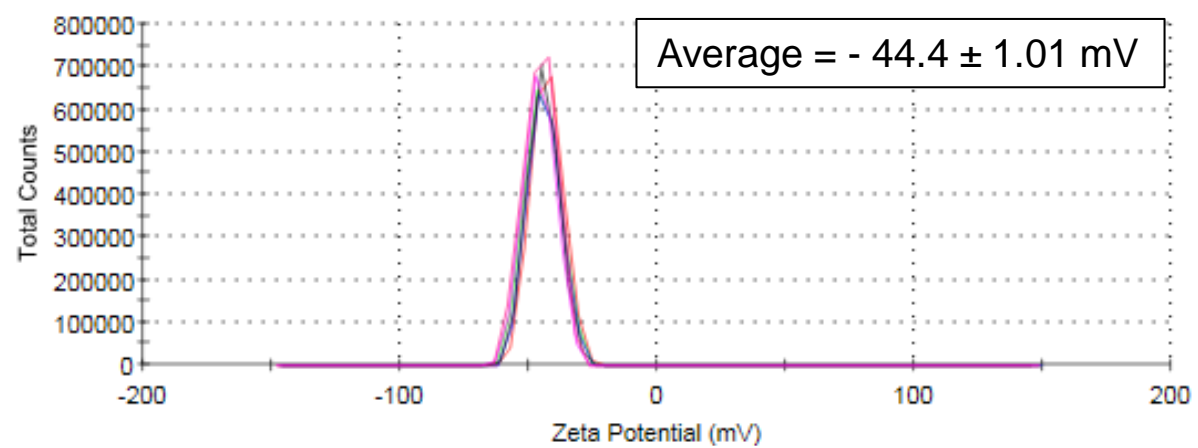

Figure S4.31. Zeta potential of CPN-1/HA.

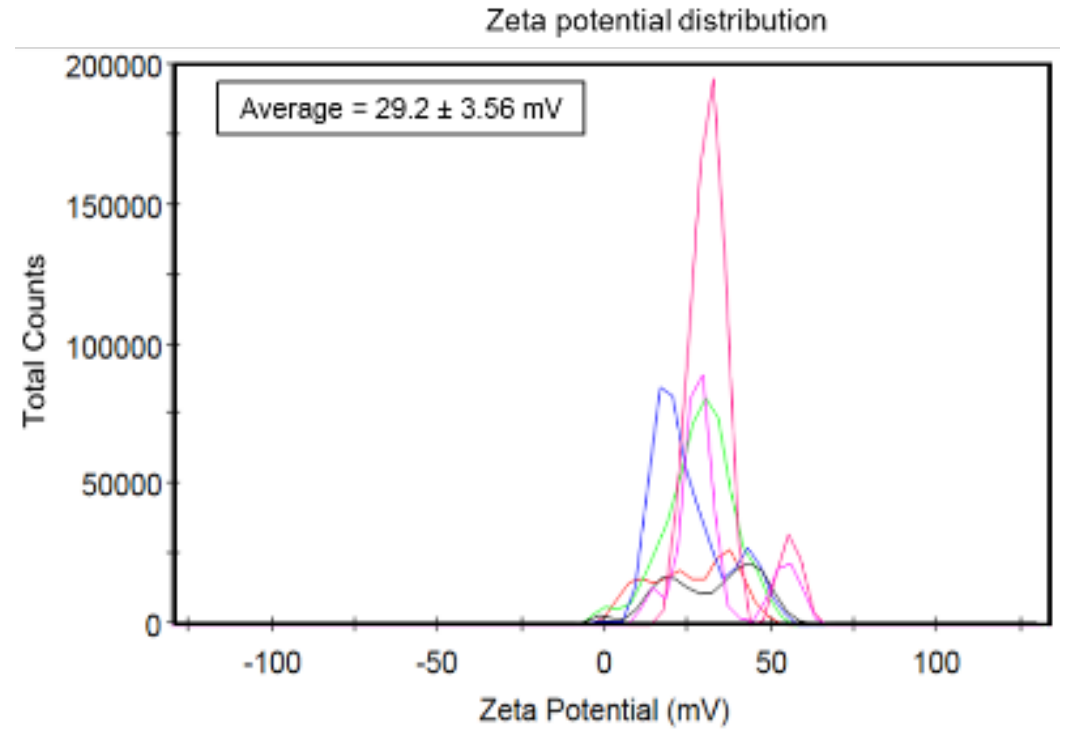

Figure S4.32. Zeta potential of CPN-2. 


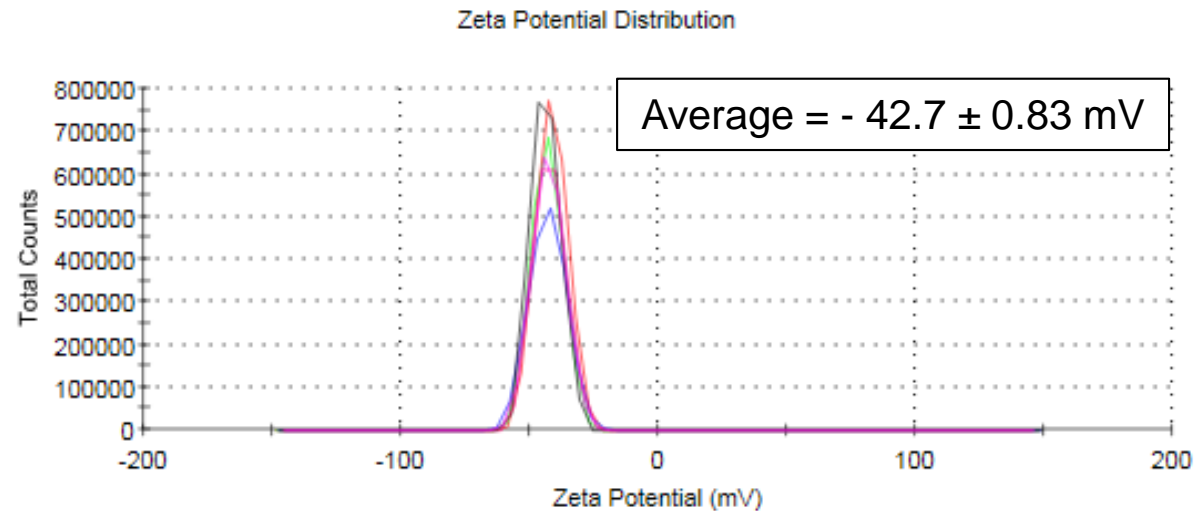

Figure S4.33. Zeta potential of CPN-2/HA.

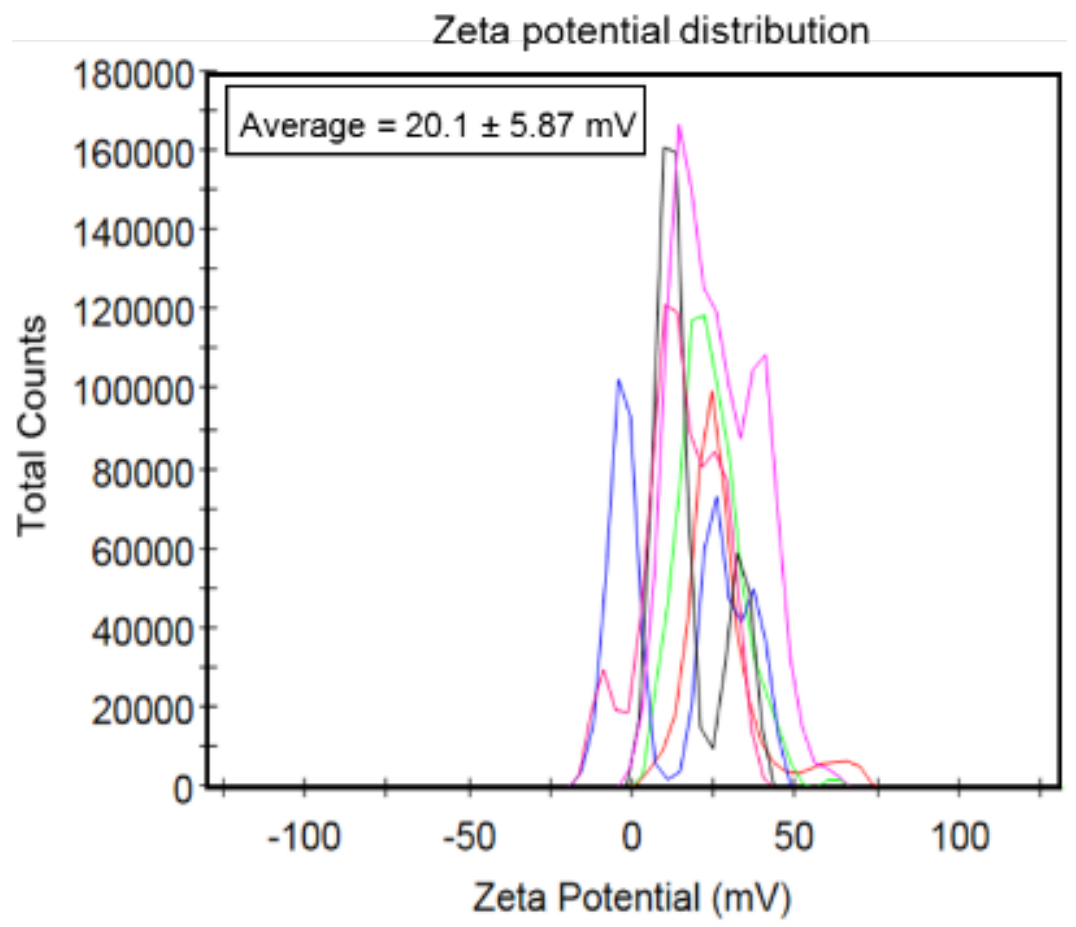

Figure S4.34. Zeta potential of CPN-3.

\subsubsection{Degradation of $\mathrm{CPN}-1$.}

To test degradation under intracellular conditions, $\mathrm{CPN}-1\left(5 \mu \mathrm{M}\right.$ in $\left.\mathrm{H}_{2} \mathrm{O}\right)$ was treated with excess glutathione (GSH) at $10 \mathrm{mM}$. The UV-vis and fluorescence emisson spectra (see Figure S34) were recorded for CPN-1 only, upon immediate addition of GSH, and after $18 \mathrm{~h}$ at $37^{\circ} \mathrm{C}$ with GSH. 


\subsubsection{General Cell Culture.}

HeLa cells (human cervical cancer, purchased from ATCC) were seeded into a 100 x 20 mm style sterile tissue culture dish (\#353003 BD Falcon, Durham, NC, USA). Then, they were cultured in complete media: Dulbecco’s minimum essential medium (DMEM)/high glucose (10 mL, HyClone, SH3024301) medium containing $10 \%$ fetal bovine serum (FBS), $100 \mathrm{U} / \mathrm{mL}$ penicillin and $100 \mu \mathrm{g} / \mathrm{mL}$ streptomycin (P/S) for $24 \mathrm{~h}$ under $5 \% \mathrm{CO}_{2}$ at $37^{\circ} \mathrm{C}$. Then, they were subcultured every 48 h. pHRhodo Dextran 10 kDa (\#P10361 Molecular probes, Life technologies, New York, USA), MitoTracker Deep Red (\#M22425 Molecular probes, Life technologies, New York, USA) and BODIPY-TR C5-ceramide-BSA complex (\#B34400 Molecular probes, Life technologies, New York, USA) were used for endosome, mitochondria, and Golgi apparatus staining, respectively. The isolation of mitochondrial and cytosolic fractions of HeLa cells was performed using an Eppendorf Centrifuge 5430R, Mitochondria Isolation Kit for for cultured cells (\#89874, Thermo Fisher Scientific), and Pierce BCA Protein Assay Kit (\#23227, Thermo Fisher Scientific). Pharmacological endocytosis inhibitors such as chlorpromazine hydrochloride (\#ALX-270-171-G005, Enzo Life Sciences, Inc.), genistein (\#AC32827-1000, Acros Organics), methyl- $\beta$-cyclodextrin (\#377110050, Acros Organics), LY2994002 (\#70920, Caymen Chemical), and cytochalasin D (\#BML-T109-0001, Enzo Life Sciences, Inc.) were purchased from Fisher Scientific.

\subsubsection{Toxicity Assay}

HeLa cells (ca. 10,000 cells per well), in $200 \mu \mathrm{L}$ of complete medium, were seeded into a 96-well plate and cultured for one day in a $5 \% \mathrm{CO}_{2}$ incubator at $37^{\circ} \mathrm{C} .4 \mathrm{mM}$ of $\mathrm{CPN}-1, \mathrm{CPN}-2$ and $\mathrm{CPN}-3$ stock solutions were prepared by dissolving dried powder in DMSO. $2 \mathrm{mM}, 1 \mathrm{mM}, 0.5 \mathrm{mM}$, and $0.1 \mathrm{mM}$ substock solutions were made by diluting with DMSO. Final concentrations of $40 \mu \mathrm{M}, 20 \mu \mathrm{M}, 10 \mu \mathrm{M}, 5 \mu \mathrm{M}$, and $1 \mu \mathrm{M}$ of CPNs were added into complete media by dilution with CPN-1, CPN-2, and CPN-3 stock solutions and then incubated for $18 \mathrm{~h}$. To measure toxicity, $10 \mu \mathrm{L}$ of MTT solution $\left(5 \mathrm{mg} \mathrm{mL}^{-1}\right.$ in PBS, CALBIOCHEM, Germany) and $90 \mu \mathrm{L}$ of complete medium were then added into each well, and the plate was further incubated for $4 \mathrm{~h}$ at $37^{\circ} \mathrm{C}$. After the formed MTT formazan crystals were dissolved in dimethyl sulfoxide $(100 \mu \mathrm{L})$, the absorbance intensity was measured by a microplate well reader (infinite M1000 PRO, TECAN, Switzerland) at $540 \mathrm{~nm}$. Relative cell viability (\%) as a function of CPN concentration was expressed as a percentage 
relative to the untreated control cells. All measurements were performed in triplicate and standard deviation was included in the error bar (see Figure S30).

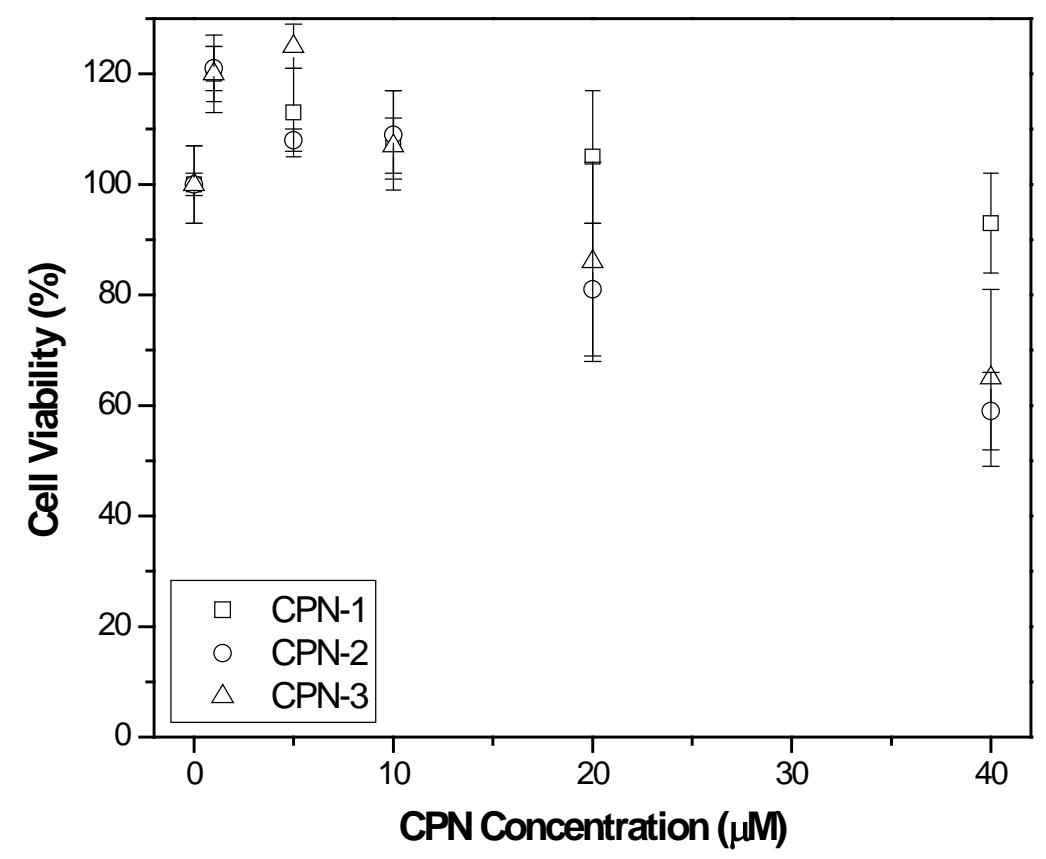

Figure S4.35. Cellular toxicity of CPN-1, CPN-2, and CPN-3.

\subsubsection{Microscopic imaging, co-localization, and time-course subcellular localization.}

10,000 HeLa cells were seeded into a glass-bottomed eight-well chamber slide (Lab-Tek Thermo Scientific) and cultured in complete media $(400 \mu \mathrm{L})$ for $24 \mathrm{~h}$ under $5 \% \mathrm{CO}_{2}$ at $37^{\circ} \mathrm{C} .80 \mu \mathrm{L}$ of $20 \mu \mathrm{M}$ CPNs in water was added to the culture medium directly, and the cells were further cultured overnight (final CPN concentration: $4 \mu \mathrm{M})$. For endosome staining, pHRhodo Dextran $10 \mathrm{kDa}(5 \mu \mathrm{M})$ was incubated for $30 \mathrm{~min}$ at $37^{\circ} \mathrm{C}$. For Golgi apparatus staining, BODIPY-TR C5 -ceramide-BSA complex (final $10 \mu \mathrm{M}$ ) was incubated for $30 \mathrm{~min}$ at $4^{\circ} \mathrm{C}$. For mitochondria staining, MitoTracker Deep Red (100 nM) was incubated for $30 \mathrm{~min}$ at $37^{\circ} \mathrm{C}$. After washing with fresh medium, the cells were further incubated for $15 \min$ at $37^{\circ} \mathrm{C}$. A $1 \mu \mathrm{L}$ aliquot of Hoechst $(5 \mu \mathrm{g} / \mathrm{mL})$ was added to the culture medium and incubated with the cells for 10 min at $37^{\circ} \mathrm{C}$, and washed two times with phosphate bu

ffered saline (PBS 10 min. Fluorescent images of the cells were obtained using a DeltaVision Elite Microscope System (Applied Precision, Issaquah, Washington, USA) equipped with bandpass filters such as blue (410-460 nm, Hoechst), green (500-550 nm, CPNs), and red (595-635nm, Golgi apparatus, endosome, and mitochondria) using a 
60X oil immersion lens (NA 1.42) and $n=1.520$ immersion oil. Top and bottom of the chosen cells was identified, and a Z-stack plot was imaged for each channel. Z-stack microscope images of each sample was obtained as described above. Colocalization analysis was conducted for three independent cells per polymer and per organelle (Golgi apparatus, endosome, and mitochondria). Colocalization analysis was conducted using the microscope software (Softworx 5.0 application, Applied Precision, Issaquah, Washington, USA). Region of interest (ROI) was selected to contain all of the cell. Pearson's Correlation Coefficient (PCC) was used to determine colocalization. Negative control of colocalization was performed by analysis of blue and green channels staining the nucleus and CPN, respectively. Three independent images of an entire cell were selected and analyzed to increase analysis objectivity.
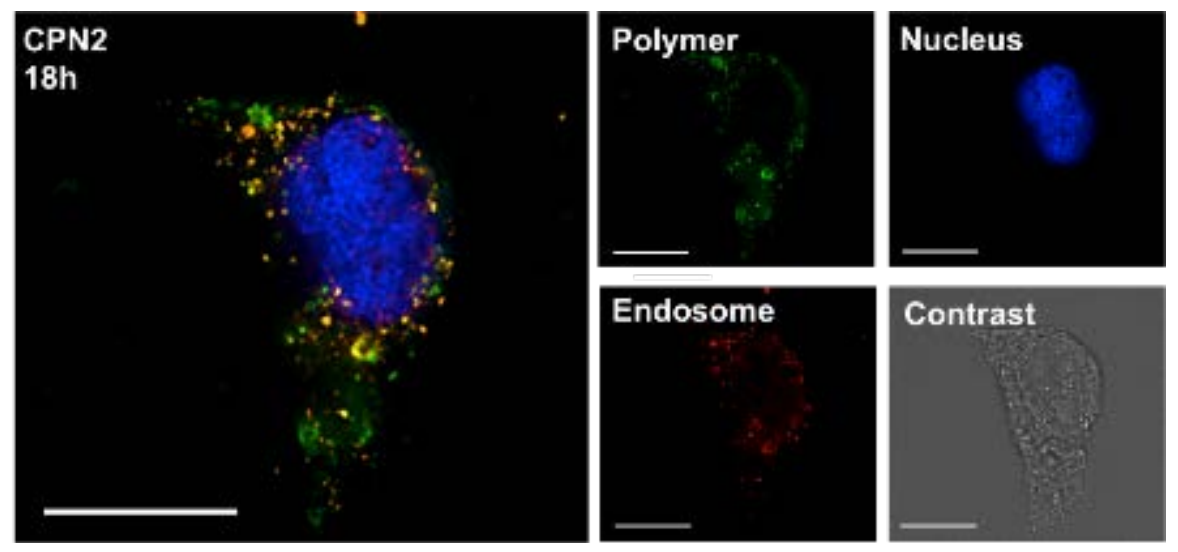

Figure S4.36. Microscopic images of HeLa cells incubated with CPN-2 for $18 \mathrm{~h}$ followed by endosome (red) and nuclear (blue) staining. The scale bar is $20 \mu \mathrm{m}$.

\subsubsection{Confocal microscopic imaging, CPN/HA co-localization, and concentration-dependent co- localization.}

HeLa cells were seeded into a 12-well plate ( 50,000/well) with glass coverslip (\#1254584, Fisher Scientific) one day prior to $\mathrm{CPN}$ treatment and cultured in complete media $(500 \mu \mathrm{L})$ for $24 \mathrm{~h}$ under $5 \% \mathrm{CO}_{2}$ at $37^{\circ} \mathrm{C}$. After cell attachment, fresh complete media $(500 \mu \mathrm{L})$ containing $50 \mu \mathrm{L}$ of $2 \mu \mathrm{M}$ or $20 \mu \mathrm{M}$ CPN in $\mathrm{H}_{2} \mathrm{O}$ (final CPN concentration: $0.2 \mu \mathrm{M}$ or $2 \mu \mathrm{M}$, see Figure S37) was added and cultured for $18 \mathrm{~h}$. For CPN/HA, fresh complete media $(500 \mu \mathrm{L})$ containing a premixed solution of $50 \mu \mathrm{L}$ of $2 \mu \mathrm{M}$ CPN in $\mathrm{H}_{2} \mathrm{O}$ (final CPN concentration: $0.2 \mu \mathrm{M}$ ) and $15 \mu \mathrm{L} 20 \mu \mathrm{M}$ HA was added and cultured for $18 \mathrm{~h}$ After incubation, cells were washed with 1X PBS and stained with MitoTracker Deep Red (50 nM) in DMEM only (no FBS or P/S) for $30 \mathrm{~min}$ at $37^{\circ} \mathrm{C}$. After washing with fresh DMEM for $15 \mathrm{~min}$ at $37^{\circ} \mathrm{C}$, cells were fixed with $4 \%$ 
paraformaldehyde for $10 \mathrm{~min}$ at room temperature. After washing with $1 \mathrm{X}$ PBS, a $1 \mu \mathrm{L}$ aliquot of Hoechst ( $5 \mu \mathrm{g} / \mathrm{mL}$ ) was added to $199 \mu \mathrm{L}$ PBS and incubated with the cells for $10 \mathrm{~min}$ at room temperature. Nuclear staining is not shown in Figure S37 due to non-specific binding of aggregated CPNs at high concentration. Cells were then washed three times with $1 \mathrm{X}$ PBS and coverslips were mounted on microscope slides (\#1125441, Fisher Scientific) using a 1:1 glycerol/PBS mounting medium. Fluorescent images of the cells were obtained using an Olympus FluorView FV1200 confocal microscope (Melville, NY USA) equipped with bandpass filters such as blue (417-477 nm, nucleus), green (513-556 nm, CPNs), and deep red (672$712 \mathrm{~nm}$, mitochondria) using a 60X oil immersion lens (NA 1.35) and $\mathrm{n}=1.519$ immersion oil. The top and bottom of the samples were identified, and a Z-stack plot was imaged for each channel. Co-localization analysis of the green (CPN) and red (mitochondria) channels was conducted for three independent images using ImageJ software (version 1.50b, U. S. National Institute of Health, Bethesda, Maryland, USA). PCC scores were calculated to determine co-localization by setting pixel threshold for the entire image per channel and per sample in order to exclude noise and background signal. ${ }^{23}$

\subsubsection{Isolation of Mitochondrial and cytosolic fractions of HeLa cells.}

HeLa cells (ca. 10,000,000) were seeded into a 100 x 20 mm style sterile tissue culture dish (\#353003 BD Falcon, Durham, NC, USA) with $10 \mathrm{~mL}$ of complete medium. After $24 \mathrm{~h}$ incubation at $37^{\circ} \mathrm{C}$, cells were treated with 5 $-\square$ thdoCQRAR, respectively, for $24 \mathrm{~h}$. After washing with PBS three times, the mitochondrial and the cytosolic fractions were isolated using a commercial kit (Mitochondria Isolation Kit for cultured cells \#89874, Thermo Fisher Scientific). Briefly, HeLa cells were isolated by scraping and washed with PBS. The kit reagent A was added followed by incubating on ice for 2 min. The kit reagent B was added followed by vortexing and incubation on ice. After 5 min of incubation, the kit reagent $\mathrm{C}$ was added and the cells were centrifuged $\left(700 \mathrm{x} g\right.$ at $\left.4{ }^{\circ} \mathrm{C}\right)$. The pellet containing nuclei and cellular debris was discarded and the supernatant was centrifuged $\left(12,000 \mathrm{x} g\right.$ at $\left.4{ }^{\circ} \mathrm{C}\right)$. The supernatant containing the cytosolic fraction was removed and the pellet composed of mitochondria was washed with the kit reagent $C$. The pure mitochondria fractions were isolated by centrifugation $\left(12,000 \mathrm{xg}\right.$ at $\left.4{ }^{\circ} \mathrm{C}\right)$. The mitochondrial fraction and the cytosolic fraction were diluted to $200 \mu \mathrm{L} 100 \%$ DMSO and $200 \mu \mathrm{L}$ of 50\% DMSO, respectively. Then the total fluorescence intensities of the mitochondrial and the cytosolic fractions resulting from the excitation 
at $455 \mathrm{~nm}$ were measured, normalized by the amount protein $(\mu \mathrm{g})$ of the fraction, and determined by BCA protein assay (Pierce BCA Protein Assay Kit \#23227, Thermo Fisher Scientific).

\subsubsection{Flow Cytometry}

HeLa cells incubated with CPNs were treated with trypan blue (Amresco Inc.) before flow cytometry measurements (Accuri C6, USA). HeLa cells incubated with $10 \mu \mathrm{M}$ of CPN for $4 \mathrm{~h}$ were treated with trypan blue $(200 \mu \mathrm{M})$ for $10 \mathrm{~min}$. After washing with $1 \mathrm{X}$ PBS buffer three times, cells were detached by trypsin/EDTA and fixed with $4 \%$ paraformaldehyde. Post-cell treatment with trypan blue was conducted for all flow cytometry analyses for entry kinetics and mechanisms.

\subsubsection{Endocytosis Inhibition Assay}

For endocytosis inhibition studies, HeLa cells were seeded into a 6-well plate ( 200,000/well) one day before CPN treatment. Cells were then treated without (control) or in the presence of pharmaceutical inhibitors chlorpromazine (24.0 mM), genistein (0.21mM), methyl- $\beta$-cyclodextrin (1.00 mM), LY294002 (0.12 mM), or cytochalasin D (0.04 mM) for 30 min before CPN treatment. After CPN treatment, cells were washed with 1X PBS three times followed by trypan blue treatment $(0.20 \mathrm{mM})$ for $10 \mathrm{~m}$. The detached cells were fixed with $4 \%$ paraformaldehyde in 1x PBS for $10 \mathrm{~m}$ and resuspended in a flow cytometry buffer (1X PBS containing $5 \%$ bovine serum albumin and $0.02 \%$ sodium azide). 10,000 events per measurement were counted and mean fluorescence intensity of CPN (FL2 channel, 518-548 nm wavelength range) was measured. Averaged mean fluorescent intensity was calculated using three independent sample sets.

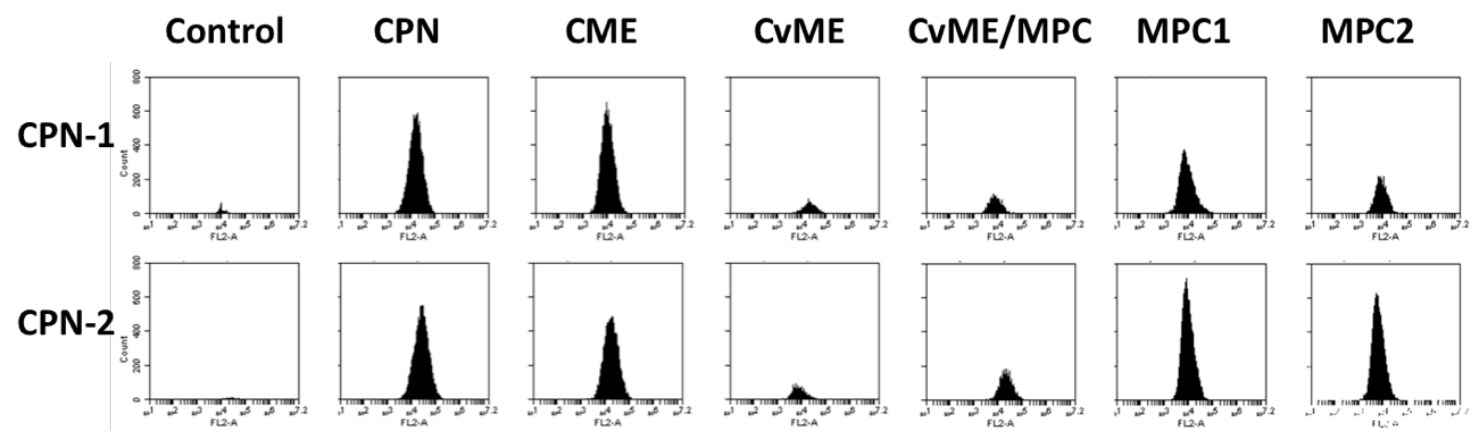

Figure S4.37. Fluorescent intensity plots obtained at the FL2 channel (518-548 nm). 
4.7 References

1. L. Feng, C. Zhu, H. Yuan, L. Liu, F. Lv and S. Wang, Chemical Society Reviews, 2013, 42, 66206633.

2. D. Tuncel and H. V. Demir, Nanoscale, 2010, 2, 484-494.

3. N. A. A. Rahim, W. McDaniel, K. Bardon, S. Srinivasan, V. Vickerman, P. T. C. So and J. H. Moon, Advanced Materials, 2009, 21, 3492-3496.

4. C. Wu, B. Bull, C. Szymanski, K. Christensen and J. McNeill, Acs Nano, 2008, 2, 2415-2423.

5. J. H. Moon, W. McDaniel, P. MacLean and L. E. Hancock, Angewandte ChemieInternational Edition, 2007, 46, 8223-8225.

6. L. H. Chen, D. W. McBranch, H. L. Wang, R. Helgeson, F. Wudl and D. G. Whitten, Proceedings of the National Academy of Sciences of the United States of America, 1999, 96, 12287-12292.

7. H. A. Ho, M. Boissinot, M. G. Bergeron, G. Corbeil, K. Dore, D. Boudreau and M. Leclerc, Angewandte Chemie-International Edition, 2002, 41, 1548-1551.

8. J. H. Moon, E. Mendez, Y. Kim and A. Kaur, Chemical Communications, 2011, 47, 8370-8372.

9. X. Feng, F. Lv, L. Liu, Q. Yang, S. Wang and G. C. Bazan, Advanced Materials, 2012, 24, 54285432.

10. T. Vokata, M. Twomey, E. Mendez and J. H. Moon, Journal of Polymer Science Part a-Polymer Chemistry, 2015, 53, 1403-1412.

11. E. Mendez and J. H. Moon, Chemical Communications, 2013, 49, 6048-6050.

12. A. Szewczyk and L. Wojtczak, Pharmacological Reviews, 2002, 54, 101-127.

13. S. S. M. Hassan and G. A. Rechnitz, Analytical Chemistry, 1982, 54, 1972-1976.

14. R. A. J. Smith, C. M. Porteous, A. M. Gane and M. P. Murphy, Proceedings of the National Academy of Sciences of the United States of America, 2003, 100, 5407- 5412.

15. A. T. Hoye, J. E. Davoren, P. Wipf, M. P. Fink and V. E. Kagan, Accounts of Chemical Research, 2008, 41, 87-97.

16. W. Davis, Z. Ronai and K. D. Tew, Journal of Pharmacology and Experimental Therapeutics, 2001, 296, 1-6.

17. V. Zinchuk, O. Zinchuk and T. Okada, Acta Histochemica Et Cytochemica, 2007, 40, $101-111$.

18. T. Vokata and J. H. Moon, Macromolecules, 2013, 46, 1253-1259.

19. D. Vercauteren, R. E. Vandenbroucke, A. T. Jones, J. Rejman, J. Demeester, S. C. De Smedt, N. N. Sanders and K. Braeckmans, Molecular Therapy, 2010, 18, 561-569. 


\section{CHAPTER V}

Dual targeting conjugated polymer nanoparticles of folate and hyaluronan receptors for bioimaging

In preparation.

Megan Twomey ${ }^{a}$, Rajib Choudhury ${ }^{a}$, Prakash Manandhar ${ }^{a}$, Irina U. Agoulnik ${ }^{b}$, and Joong Ho Moon ${ }^{*}$

aDepartment of Chemistry and Biochemistry, Biomolecular Science Institute, Florida International University, Miami, Florida, 33199, USA

bDepartment of Cell Biology and Pharmacology, Herbert Wertheim College of Medicine, Florida International University, 11200 SW 8th St. Miami, FL 33199, USA. 


\subsection{Abstract}

Red emissive conjugated polymer nanoparticles (CPNs) grafted with folic acid (FA)-modified poly(ethylene glycol) (PEG 2000 ) side chains were fabricated and complexed with hyaluronic acid (HA) for improved targeting and labelling of HeLa cells. Modulation of uptake kinetics and amount of internalized CPNs was achieved by electrostatic complexation as measured by flow cytometry and visualization by high resolution fluorescence microscopy. These dual ligand-targeting CPNs designed for folate and hyaluronan receptors are effective for labelling and bioimaging.

\subsection{Introduction}

Conjugated polymer nanoparticles (CPNs) possess excellent light harvesting and light amplifying properties owing to their delocalized electronic structure along a $\pi$-conjugated backbone, making them excellent materials for a variety of applications, such as optoelectronic devices, sensing, imaging, and drug delivery. ${ }^{1-}$ 4, 7, 28 These CPNs have become of significant interest in therapeutics and diagnostics (theranostics) as a consequence of their desirable properties including low cytotoxicity, biocompatibility, facile synthesis, high sensitivity and superior photostability. ${ }^{5}$ Structural modifications of CP side chains to incorporate ionic and hydrophilic moieties (e.g.; charged functional groups and polyethylene glycol chains) allow for tunable biophysical properties for improved biocompatibility and functionalized performance. ${ }^{10,31}$ Recently, CPNs have been utilized as sensitive tumor imaging probes for both in vitro and in vivo applications. ${ }^{6,8,27,29}$ More importantly, efficient delivery of CPNs to tumor cells has been achieved in theranostics and bioimaging by utilizing cell surface receptors. ${ }^{8,26}$ Rapid delivery of desired materials in large quantities to a wide variety of tumor cells has become feasible using targeting delivery vehicles. ${ }^{9}$ In these approaches, ligands are covalently attached to CPNs to selectively interact/bind with overexpressed receptors on the cell surface. Although these targeting approaches improves selectivity of CPNs towards tumor cells, it still suffers from the fact that these nanomaterials (e.g., drug candidates, imaging agents) are often not highly tumor specific, and cause toxicity to normal cells. To overcome this problem, some research groups have utilized a dual targeting approach to improve the tumor selectivity of chemotherapy and imaging agents aiming to simultaneously target multiple receptors on the cell surface. ${ }^{21}$ In their designs, two ligands are conjugated to the cargo via covalent 
attachment. Upon interaction with cancer cells, each ligand binds to their specified receptors resulting in improved selectivity while sparing unintentional targets (i.e., healthy cells). ${ }^{22}$

To address these issues, “dual targeting” CPNs have been designed for improved labelling and bioimaging. In our approach, folic acid (FA) binding folate receptors (FR) and hyaluronic acid (HA) binding hyaluronan receptors were chosen as targeting components as these will ultimately increase interactions between CPNs and tumor cells. Folate receptors are composed of a glycosylphosphatidylinositol (GPI)-linked membrane glycoprotein that exists in two membrane-bound isoforms, FR- $\alpha$ and FR- $\beta$, which are overexpressed in ovarian, breast, endometrial, colorectal, and lung carinomas. ${ }^{23}$ Folic acid is easily internalized into the cytoplasm as it has a very high affinity to these receptors $\left(K_{\mathrm{d}} \sim 1 \mathrm{nM}\right)$, which is an advantage for efficient intracellular delivery, ${ }^{24}$ The binding affinity of FA to FR has been shown to remain very high even when conjugated to other materials. ${ }^{11}$ Conjugation of FA to macromolecules presents synthetic issues given its hydrophobic nature and limited solubility. However, FA has been shown to take on the properties of the material after conjugation. ${ }^{32}$ Hyaluronic acid is an anionic, hydrophilic, and biodegradable high molecular weight polymer ( $10^{6}$ to $10^{7} \mathrm{Da}$ ) that interacts through several receptors, including CD44 (Cluster Determinant 44), RHAMM (receptor for HA-mediated motility), HARE (HA receptor for endocytosis), and ICAM-1 (Intercellular Adhesion Molecule-1), which are overexpressed in tumor cells and multiple carcinomas. ${ }^{19,20}$ Hyaluronic acid is an excellent candidate for cancer cell targeting and has been used for more than 30 years as a drug carrier and/or targeting moiety for biomaterials to target cancer cells.

Reported herein are highly fluorescent far-red emissive $(600-800 \mathrm{~nm})$ conjugated polymeric nanomaterials bearing two types of targeting ligands: covalently attached FA and electrostatically complexed HA. The CP contains a benzothiadiazole (BT)-doped poly(p-phenyleneethynylene) (PPE) hydrophobic backbone, which emits in the far-red region. In general, the hydrophobic backbone of CPs causes polymer chains to aggregate in aqueous solvent, resulting in decreased quantum yield and undesired nonspecific binding. To overcome these drawbacks, the side chain of the polymer was modified with a quaternary ammonium group in a onepot synthesis, and poly(ethylene glycol) ( $\mathrm{PEG}_{2000}$ ) was conjugated onto the side chain of the CPs to improve water solubility, reduce undesirable aggregation, and eliminate nonspecific interactions with cells. Folic acid and PEG 2000 were attached to the CP side chain via simple "click reaction”, whereas HA was incorporated 
into the polymers via mixing in solution, by taking advantage of electrostatic and hydrophobic interactions. Thus, the design principle reduces tedious synthetic effort to conjugate high molecular weight HA onto PPE. Moreover, non-covalent complexation provided the freedom of fine-tuning the outer surface of the nanomaterials with various HAs (i.e.; molecular weight and molar ratio).

Chapter V describes the synthesis, characterization, and effectiveness of a conjugated polymer-based dualtargeting imaging system. The hypothesis was tested in HeLa cells, which express both folate and hyaluronan receptors. The results demonstrated that the dual-targeting imaging system can indeed be employed to image cancer cells with heightened efficiency.

\subsection{Results and Discussion}

The grafted copolymers for CPN-PEG 2000-FA and CPN-PEG 2000, (shown in Figure 5.1) were prepared utilizing azide functionalized PPE copolymers coupled with alkynyl end of $\mathrm{PEG}_{2000}-\mathrm{FA}$ and $\mathrm{PEG}_{2000}$, respectively (see experimental). Grafting efficiency of $\mathrm{PEG}_{2000}$-FA to the copolymer was determined to be $40 \%$ resulting in a triblock copolymer (see Figure S5.1). The grafting of $\mathrm{PEG}_{2000}$ only was much more efficient with all of the azide functionalized side chain converted resulting in a 1:1 copolymer. In addition, the one-pot conversion to quaternary ammonium salt was only $50 \%$ because of synthetic conditions to control benzothiadiazole incorporation (see Figure S5.4) After purification via dialysis and followed by freeze drying, both $\mathrm{PEG}_{2000}$ only and FA-attached $\mathrm{PEG}_{2000}$ grafted conjugated polymers were used to construct nanostructures through a self-assembly process. The polymers were first dissolved in good solvent (DMSO) then added to deionized water under vigorous stirring to induce self-assembly.
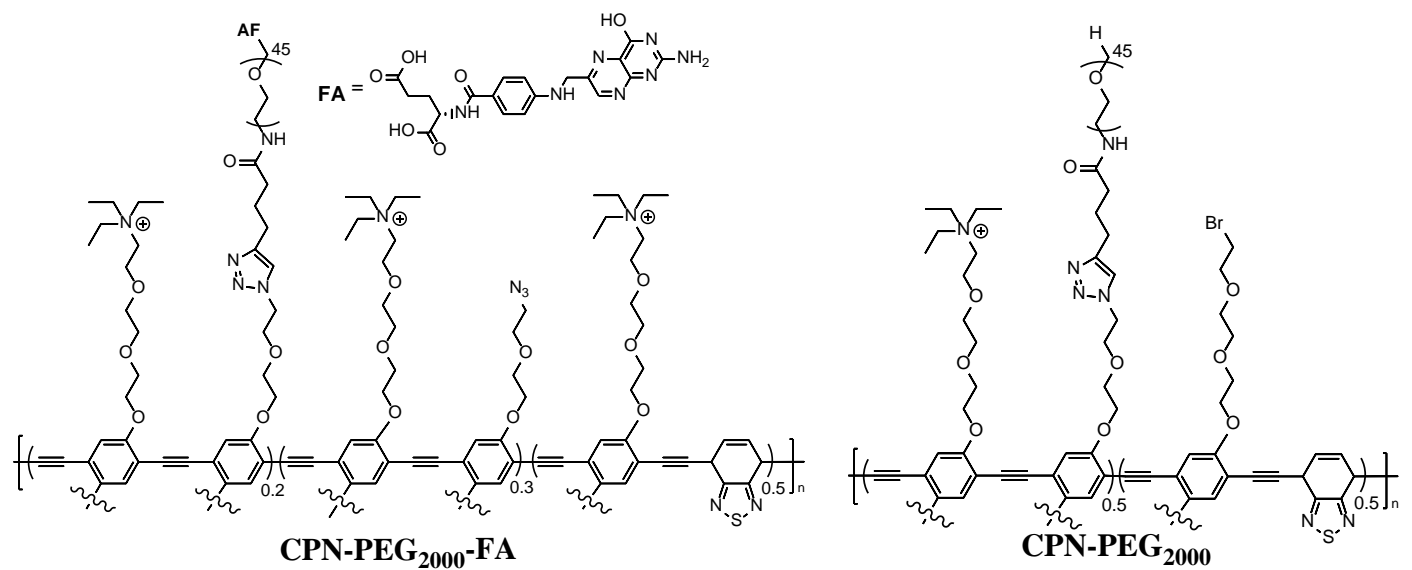

Figure 5.1. Chemical structures CPNs grafted with folic acid-modified $\mathrm{PEG}_{2000}\left(\mathrm{CPN}-\mathrm{PEG}_{2000}-\mathrm{FA}\right)$ and $\mathrm{PEG}_{2000}$ only (CPN-PEG 2000 ). 
The UV-vis absorption and emission spectra of CPN-PEG 2000-FA and CPN-PEG 2000 in water are shown in Figure 5.2. The absorption maxima for CPN-PEG 2000-FA and CPN-PEG 2000 are 488 and 483 nm, respectively. These values are $\sim 30 \mathrm{~nm}$ higher than the absorption maxima in THF, indicating hydrophobic main chain induced aggregation and lengthening of the conjugated chains (see Figure S5.21). The emission maxima for CPN-PEG 2000-FA and CPN-PEG 2000 in water are $618 \mathrm{~nm}$ and $620 \mathrm{~nm}$, respectively. The significantly red emission compared to structurally similar PPE polymers without BTz units suggests efficient donor-acceptor type energy transfer from PPE unit to BTz unit. Moreover, a slight increase in emission maxima ( 20 nm) in water compared to organic solvents such as chloroform, DCM and THF indicates minimal chain-chain type aggregation in water. The emission quantum yields $(\Phi)$ for CPNPEG $2000-F A_{\text {and }} \mathbf{C P N}_{\mathbf{P}} \mathbf{P E G}_{2000}$ are 0.55 and 0.50, respectively, calculated using coumarin 6 in ethanol as the standard. The incorporated bulky side-chains (i.e.; $\mathrm{PEG}_{2000}$, FA, and quaternary ammonium moieties) likely sterically hinder excessive inter-chain aggregation in water. Compared to the more green and red emissive conjugated polymers typically used for nanoparticle fabrication and imaging, CPN-PEG 2000-FA and CPN-PEG 2000 have relatively higher quantum yields in water, which is ideal for NPs used in bioimaging.
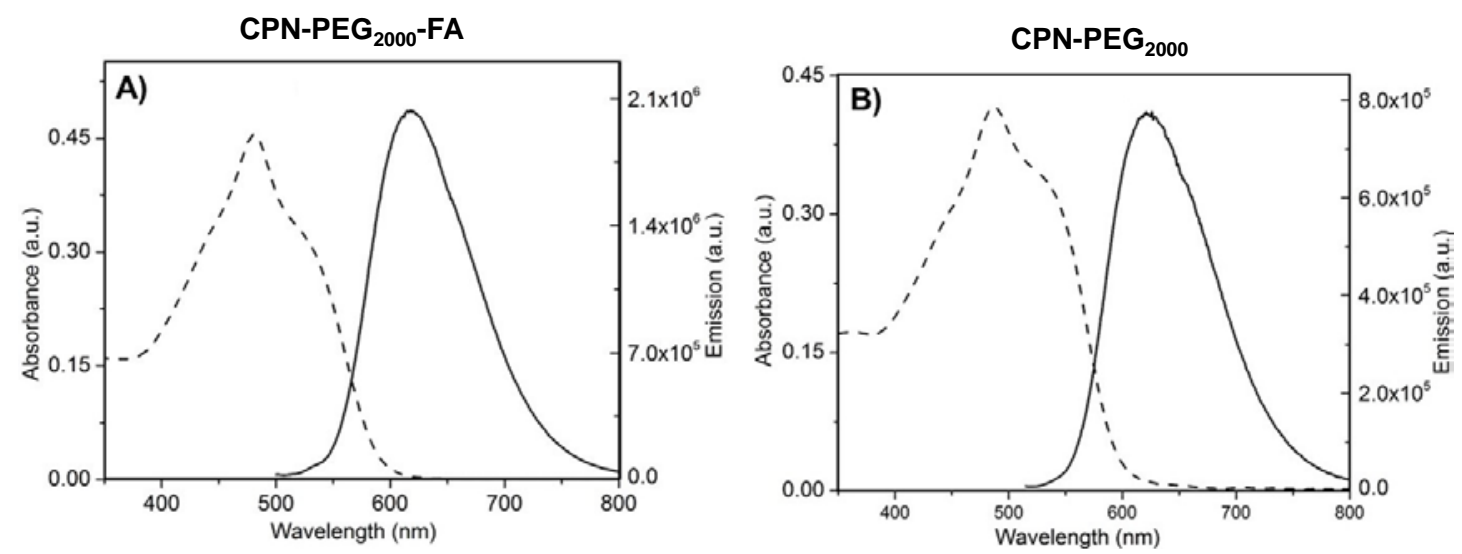

Figure 5.2. Absorption and emission spectra for (a) CPN-PEG 2000-FA and (b) CPN-PEG 2000 in water $($ Excitation $=480 \mathrm{~nm}$, slit width $=3 \mathrm{~nm}$, integration time $=0.1 \mathrm{~s})$.

The resulting NPs had diameters ranging from 105 to 108 nm, as determined by NTA analysis (see Figure 5.6). The side chains with the nonionic diethylene oxide moiety and hydrophilic PEG $_{2000}$ apparently stabilize the surface of the NPs, thereby hindering aggregation and precipitation. Typically, hydrophobic NPs aggregate in water as a mechanism of water exclusion from the hydrophobic surface. It was expected that the 
CPNs fabricated from polymers containing hydrophilic side chains (i.e., $\mathrm{PEG}_{2000}$ ) and ionic side chains (i.e.; quaternary ammonium salt) will remain stable over time. To more thoroughly asses the stability of these NPs, the size distribution of the colloidal suspension was checked after one month. No significant change to the size distribution of the NPs, suggesting NPs are highly stable in water. High colloidal stability of the CPNs and uniform particle formation were also confirmed by zeta potential measurements. Both $\mathbf{C P N}_{\mathbf{P}} \mathbf{P G}_{\mathbf{2 0 0 0}}$ FA $(\zeta+89.7 \pm 2.21 \mathrm{mV})$ and $\mathbf{C P N}-\mathbf{P E G}_{2000}(\zeta+44.7 \pm 1.02 \mathrm{mV})$ have very high positive surface charge with narrow charge distribution in water (shown in Figure S5.22). The high positive charges can be attributed to the presence of quaternary ammonium ions on the nanoparticle surface. While the high zeta potentials of CPNs may cause issues upon interactions with cellular proteins, the particle surface can be further modified with optimization of quaternary ammonium salt incorporation.

Both CPNs (FA functionalized and non-functionalized) showed limited cytotoxicity after $18 \mathrm{~h}$ of incubation, only decreasing to $80 \%$ viability upon treatment with $40 \mu \mathrm{M}$ CPN-PEG 2000-FA (see Figure S5.23). Moreover, this concentration of CPNs was approximately seven times higher than that of CPNs used for in vitro experiments. The minimal toxicity of the CPNs towards HeLa cells, in spite of presence of ionic side chains, was attributed to the presence of biocompatible PEG $_{2000}$ in the outer layer of the polymer. ${ }^{5}$ Toxicity of the CPN/HA complexes were not measured, since HAs are known to be nontoxic and have been used to reduce toxicity of polyethyleneimine (PEI) cationic polymers.

Flow cytometry experiments were performed to quantitate the efficacy of CPNs for dual-targeting in tumor cells. The CPNs were complexed with HA at different ratios using 40 and $100 \mathrm{kDa} \mathrm{HA}$, as it is known that molecular weight of HA plays a role in receptor interactions and biodistribution. ${ }^{27}$ It was expected that the molar ratio between CPN and HA will be an important factor to optimize when using different MW HAs. Our previous work indicated that complexation of cationic CPNs with $30 \mu \mathrm{M}$ of $100 \mathrm{kDa}$ HA increased cancer cell labelling and this served as a starting point for screenings. ${ }^{29}$ To improve maximum fluorescence signal from cell internalized CPN/HA complexes, several ratios of CPN/HA complexes were investigated. The HeLa cells were incubated for $18 \mathrm{~h}$ in presence of CPN/HA complexes at molar ratios of 1:1, 1:2, and 1:5. After incubation, cells were treated with trypan blue $(200 \mu \mathrm{M})$ to quench fluorescence originating from CPNs attached to the cell membrane, which was verified in solution (Figure S5.24). Trypan blue is known to 
absorb energy in the wavelength range that CPNs emit fluorescence, and treatment is an established method to quench extracellular fluorescence. ${ }^{33-36}$ Mean fluorescence intensities (average of three replicates) determined by the scattering of control cells (Figure S5.25) were plotted against different ratios of CPN/HA complexes with 40 and $100 \mathrm{kDa} H A$ as shown in Figure 5.3. From these results, it was concluded that 40k HA showed higher fluorescence for internalized CPN/HA complexes. The HeLa cells exhibited maximum fluorescence intensity at $\mathbf{C P N}-\mathbf{P E G}_{\mathbf{2 0 0 0}} \mathbf{- F A} / \mathrm{HA}$ ratio of 1:1, with a two-fold increase using 40k HA. Any excess of HA further reduced the fluorescence intensity, which could be the result of excess HA and negative charges that will not favor uptake of the CPNs at the cell surface. The control CPN-PEG 2000 showed maximum fluorescence intensity at 1:2 ratio $\left(\mathbf{C P N}_{\mathbf{P}} \mathbf{P E G}_{\mathbf{2 0 0 0}} / \mathrm{HA}\right)$, however, the increase in fluorescence was very minimal compared $\mathbf{C P N}-\mathbf{P E G}_{\mathbf{2 0 0 0}} \mathbf{- F A}$. A similar trend was observed for $100 \mathrm{kDa}$ HA, with a greater reduction in fluorescence compared to $40 \mathrm{kDa} H A$. Moreover, complexation with $100 \mathrm{kDa}$ HA gave viscous

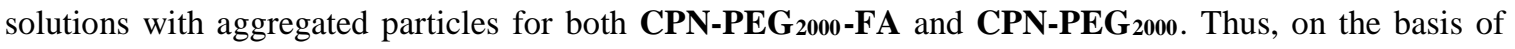

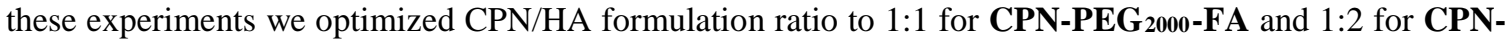
PEG 2000 complexed with 40 kDa HA for further studies.
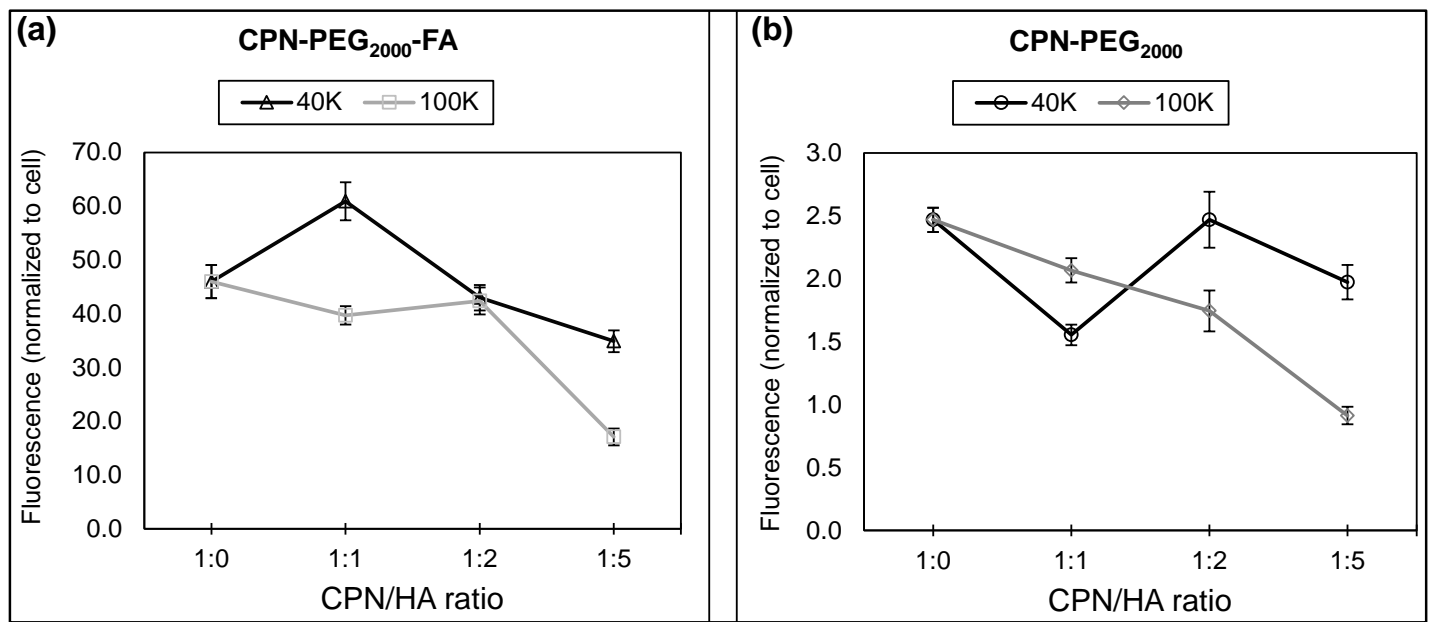

Figure 5.3. Cellular uptake of CPN-PEG 2000-FA (a) and CPN-PEG 200 (b) complexed with 40 kDa and 100 $\mathrm{kDa} \mathrm{HA}$ at various ratios after $18 \mathrm{~h}$ incubation. Error bars represent standard deviation from $\mathrm{n}=3$.

The effectiveness of the dual targeting CPNs was investigated in a time course experiment. It was anticipated that internalization of the $\mathbf{C P N}-\mathbf{P E G}_{\mathbf{2 0 0 0}} \mathbf{- F A} / \mathrm{HA}$ complex would occur via both folate and hyaluronan receptors, and these events would enhance uptake of the complex resulting in an increased fluorescence signal 
from inside the cells. Figure 5.4 shows a comparison plot for fluorescence associated with internalized CPNs and CPN/HA complexes at different incubation time. In the case of CPN-PEG 2000-FA only, maximum fluorescence was obtained after $18 \mathrm{~h}$ of incubation. Typically, folate receptor mediated endocytosis is rapid ( $\sim .5 \mathrm{~h}$ ) for free FA and FA conjugated to small molecules, ${ }^{11}$ but when FA is conjugated to macromolecules (i.e., PPEs), maximum uptake can range 4-24 h. ${ }^{12,13}$ Upon complexation of $\mathbf{C P N - P E G}$ 2000-FA with HA (1:1) ratio, the maximum fluorescence was obtained at $8 \mathrm{~h}$, with $6 \mathrm{X}$ increase in intensity compared to $\mathbf{C P N}$ $\mathbf{P E G}_{2000}$-FA only. The maximum uptake time of $8 \mathrm{~h}$ has been for reported for similar HA-conjugated NPs under various formulations. ${ }^{14}$ This observation indicates an increase in CPN uptake in shorter time because of hyaluronan mediated endocytosis, and more importantly, modulation of endocytosis can be achieved for enhanced uptake by simple mixing with HA. It is hypothesized that the substantial increase in uptake of the CPN-PEG $2000-\mathbf{F A} /$ HA complex results from increased interactions through hyaluronan receptors as HA has been shown to modulate multiple receptors during mediated endocytosis. ${ }^{14}$ By utilizing HA with CPN$\mathrm{PEG}_{2000}-\mathrm{FA}$, it is also hypothesized that a cooperative effect contributes to the substantial uptake increase due to multiple ligands/receptors and CPNs at the cell surface. ${ }^{18}$ The cooperative effect can be attributed to the availability of both FA and HA on the outside of CPN/HA complex for receptor -ligand interactions. It is assumed that the CPNs form random complexes with HA as there are no significant changes in UV absorption and emission spectral shapes upon complexation with HA, indicating no significant structural reorganization of polymer chains (see Figure 5.5). There is also an increase in particle size and broadening of particle size distributions (see Figure 5.6), which indicate random complex formation. Similar uptake values at $18 \mathrm{~h}$ for both $\mathbf{C P N}_{\mathbf{P}} \mathbf{P E G}_{\mathbf{2 0 0 0}} \mathbf{- F A}$ and $\mathbf{C P N}_{\mathbf{P}} \mathbf{P E G}_{\mathbf{2 0 0 0}} \mathbf{- F A} / \mathrm{HA}$ are speculated to be driven by FR mediated endocytosis with recycling of receptors back to the cell surface ${ }^{11}$ Time course experiments with CPN-PEG 2000 exhibited a more intense signal (3X) from cells upon incubation with mixing with HA (1:2) for $4 \mathrm{~h}$, with similar maximum uptake time. This suggests that the kinetics of endocytosis for both CPN$\mathrm{PEG}_{2000}$ and $\mathrm{CPN}-\mathrm{PEG}_{2000} / \mathrm{HA}$ are driven by factors independent from hyaluronan mediated endocytosis. Both CPN/HA complexes display a decrease in uptake after maximum uptake time, which could be attributed to inefficient endosomal escape at longer incubation times. Further studies are needed to better understand the kinetics of the dual ligand system. 

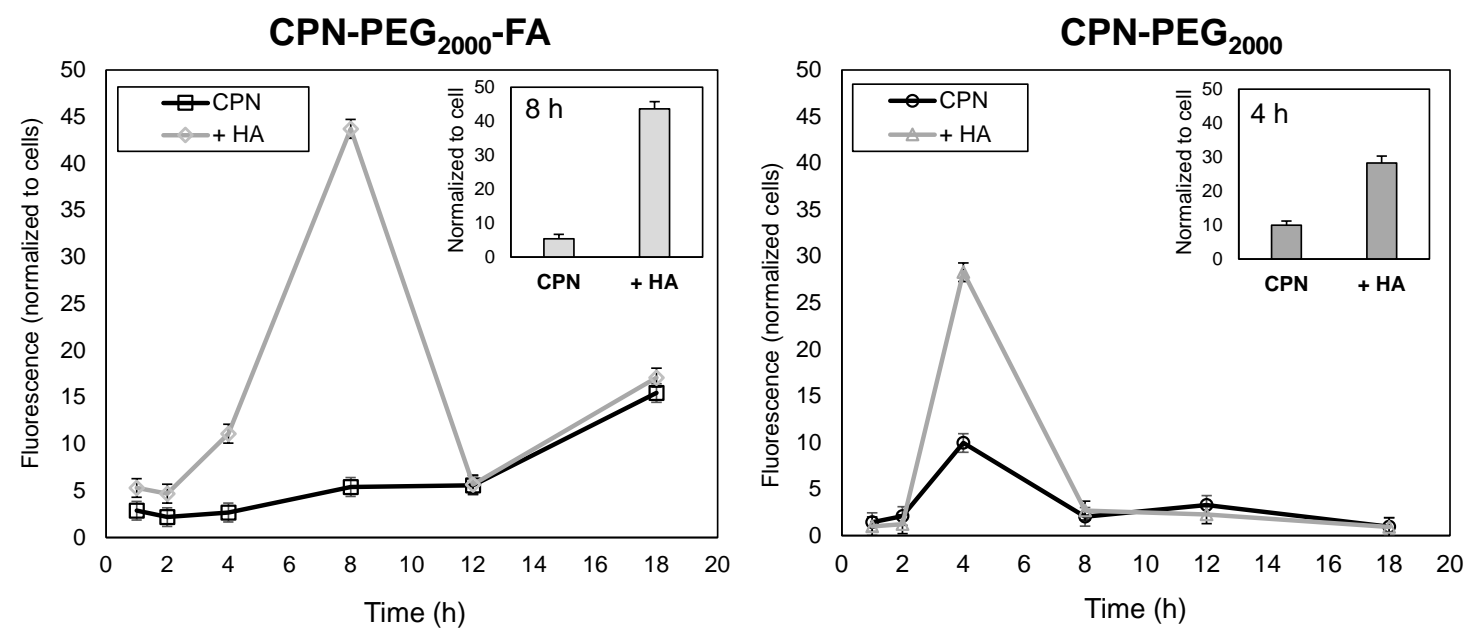

Figure 5.4. Plot of fluorescence intensities of CPN vs incubation time at optimized formulation of CPN/HA showing, (a) six-fold enhancement of $\mathbf{C P N}-\mathbf{P E G}_{2000}-\mathbf{F A}$ uptake (8h vs 18h), (b) three-fold enhancement of CPN-PEG 2000 uptake at $4 \mathrm{~h}$. Error bars represent standard deviation from $\mathrm{n}=3$.
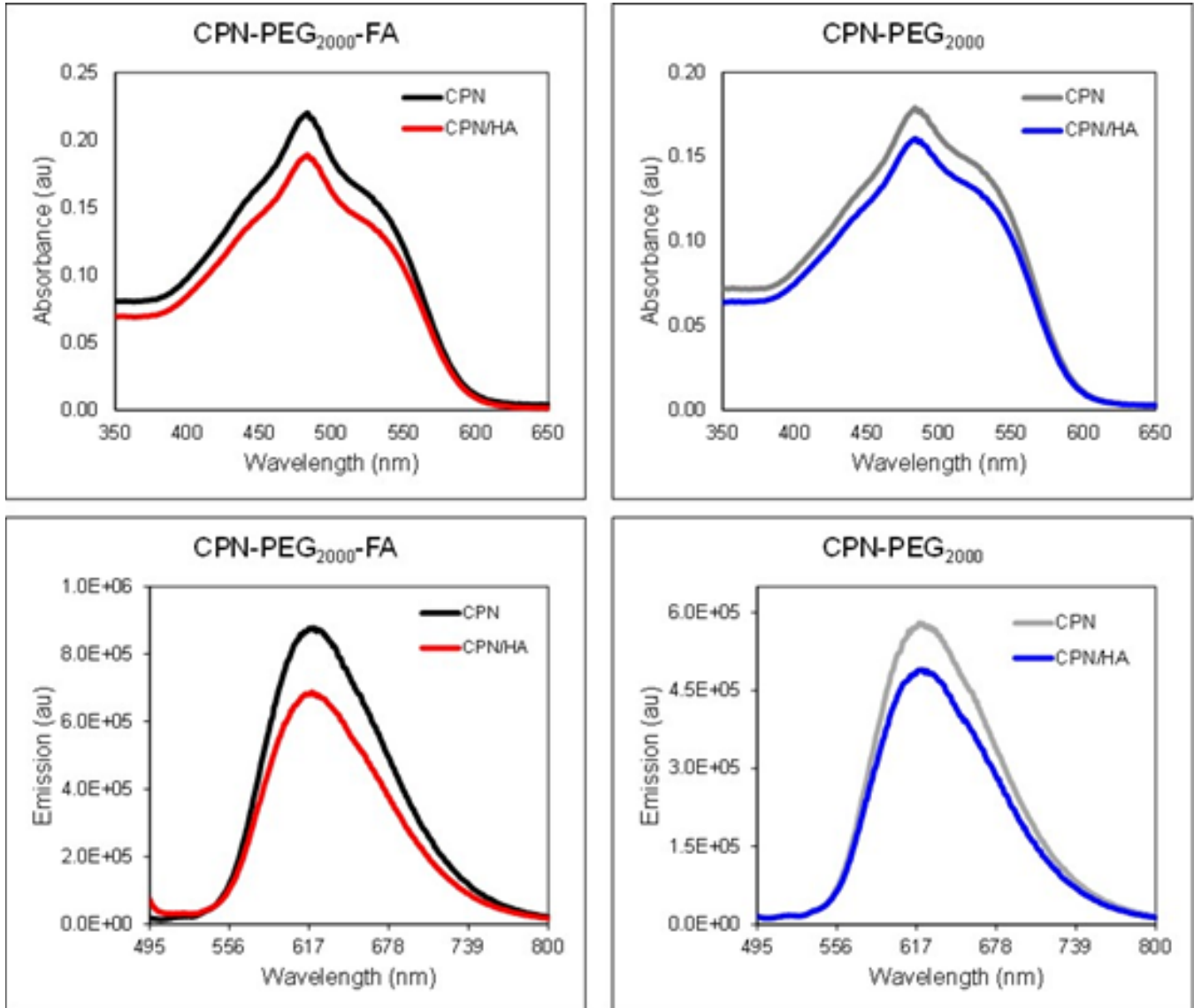

Figure 5.5. UV-absorption (top row) and emission (bottom row) spectra for $\mathbf{C P N}^{-P E G}$ 2000-FA (left column) and CPN-PEG 2000(right column) complexed with $40 \mathrm{kDa}$ HA at 1:1 and 1:2 molar ratios, respectively. 
(a)

\begin{tabular}{|l|c|}
\hline \multicolumn{1}{|c|}{ Sample } & Mean size $(\mathrm{nm})$ \\
\hline CPN-PEG $_{2000}$-FA & $105.5 \pm 1.4$ \\
\hline CPN-PEG & 2000 \\
-FA/HA & $202.6 \pm 2.2$ \\
\hline CPN-PEG $_{2000}$ & $107.9 \pm 4.5$ \\
\hline CPN-PEG $_{2000} / \mathrm{HA}$ & $233.4 \pm 22.7$ \\
\hline
\end{tabular}

(b)
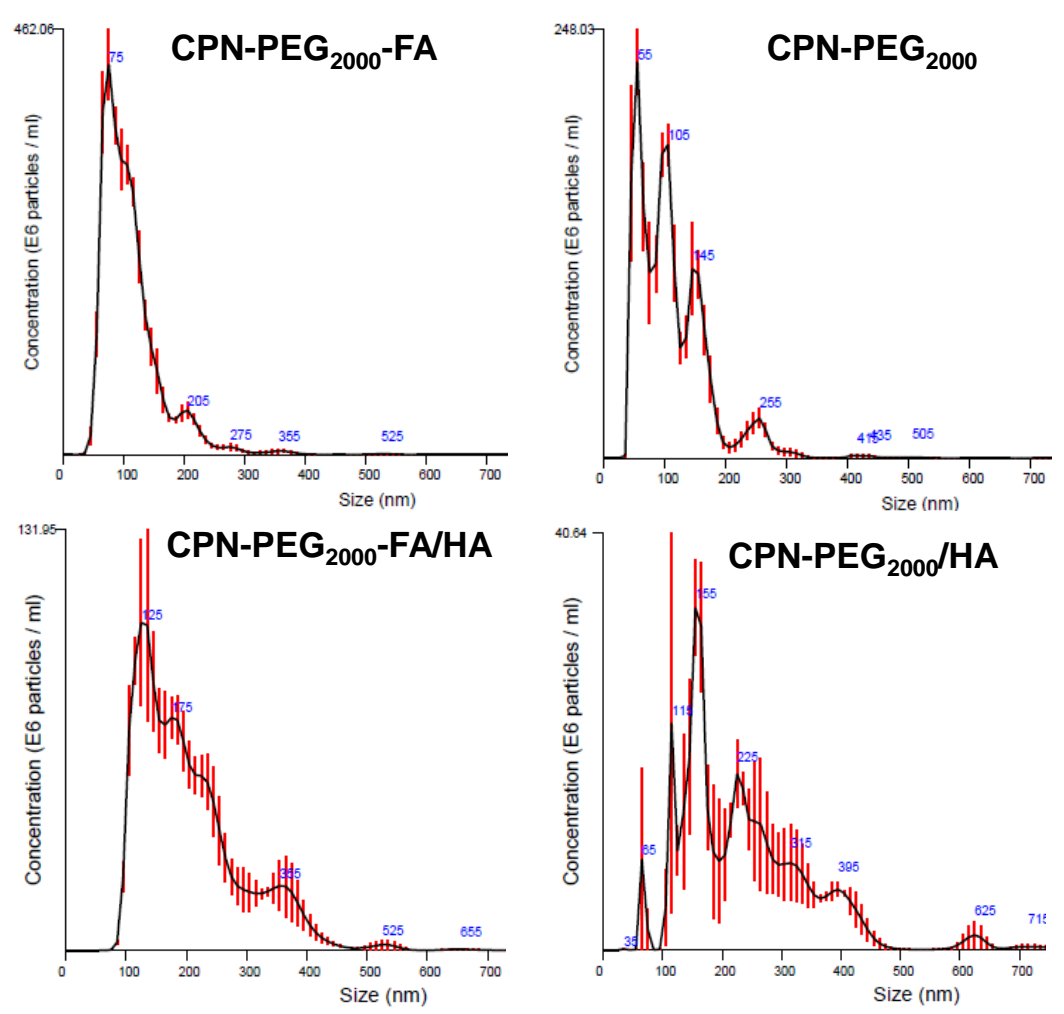

Figure 5.6. Mean sizes (a) and size distributions (b) as measured by NTA of CPN/HA complexes.

The results from the previous flow cytometry uptake studies and microscopic imaging experiments suggest different endocytosis mechanisms involved during uptake of CPNs and CPN/HA complexes. Mechanistic studies for CPN and CPN/HA complexes were performed using several known endocytic inhibitors and competitive inhibition by adding free FA and free HA to the culture medium prior to CPN treatment. As

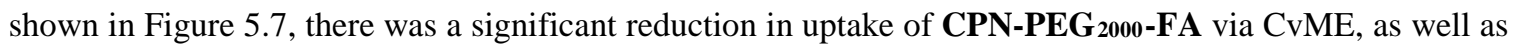
reduced uptake via MPC and excess FA. These results are expected as FR are known to be on the cell surface and as well as within caveolae. ${ }^{16,23}$ Similar pathways were inhibited for CPN-PEG 2000-FA/HA, with 
significant reduction from excess HA. The inhibition studies indicate that $\mathbf{C P N}-\mathbf{P E G} \mathbf{2 0 0 0}_{\mathbf{2}} \mathbf{- F A}$ follows typical folate-mediated endocytosis and when electrostatically complexed with HA, displays both folate and hyaluronan receptor mediated endocytosis. The control $\mathbf{C P N}-\mathbf{P E G}_{2000}$ displayed reduced update via CME, CvME, and MPC pathways, whereas $\mathbf{C P N}_{\mathbf{P}} \mathbf{P E G}_{\mathbf{2 0 0 0}} / \mathrm{HA}$ showed reduced uptake via MPC and excess HA. These results suggest that $\mathbf{C P N}-\mathbf{P E G}_{2000}$ has multiple endocytosis pathways, which could be attributed to the hydrophilic nature of the $\mathrm{PEG}_{2000}$ side chain that allows for longer circulation time,${ }^{17}$ and may not be as specific as $\mathbf{C P N}-\mathbf{P E G}_{\mathbf{2 0 0 0}} \mathbf{- F A}$. While it is evident that there are different uptake mechanisms for CPNs only and CPN/HA complexes, mechanistic studies did not provide definitive results, which could be the explained by the differences in optimal uptake times and the time dependence of intracellular trafficking.
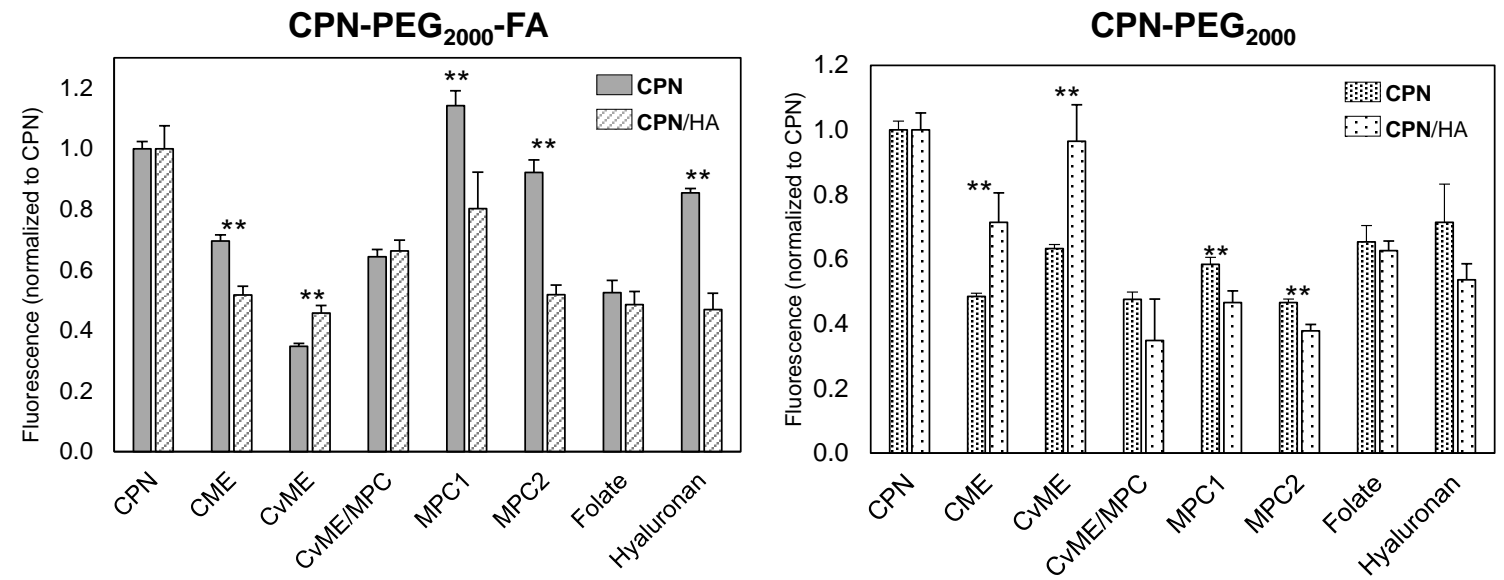

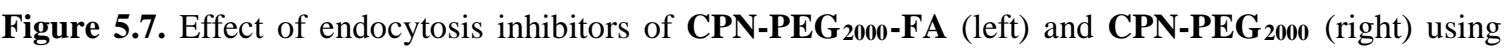
chlorpromazine $(24 \mu \mathrm{M})$ for clathrin mediated endocytosis $(\mathrm{CME})$, genistein $(210 \mu \mathrm{M})$ for caveolae mediated endocytosis (CvME), methyl- $\beta$-cyclodextrin $(1000 \mu \mathrm{M})$ for CME and macropinocytosis (CvME/MPC), LY294002 $(120 \mu \mathrm{M})$ for macropinocytosis (MPC1), or cytochalasin D $(0.04 \mathrm{mM})$ for macropinocytosis (MPC2) incubated 30 minutes before CPN treatment. For blocking any potential pathways of FA (folate) and HA (hyaluronan), cells were treated with excess FA (1 mM) and HA (100 kDa, $1.5 \mathrm{mM})$, respectively, for one hour before CPN treatment. Average values are reported $(n=3)$ with standard deviation represented in error bars. ${ }^{*} \mathrm{p}=0.01$.

High resolution fluorescence microscopy was used to visualize the distribution of internalized CPNs. After incubation of cells with CPNs and CPN/HA complexes for desired time, cells were washed with PBS, fixed with $4 \%$ paraformaldehyde, and then treated with Hoechst dye to differentiate nuclei. After a final washing with PBS, the fixed cells were observed under a high resolution microscope. For each sample top and bottom of the selected cells were identified, and a Z-stack plot was imaged for each channel. Although some internalization of CPNs was observed after just $2 \mathrm{~h}$ incubation, the optimal incubation time for each CPN/HA complex is reported here, which is $8 \mathrm{~h}$ and $4 \mathrm{~h}$ for CPN-PEG 2000-FA/HA and CPN-PEG 2000/HA, 
respectively. As shown in Figure 5.8a, the overlay image obtained from the blue and red channels for CPNPEG 2000-FA showed red fluorescence dots in the cytoplasm and surrounding the nucleus, as well as outside of the cell. The incorporation of $\mathrm{PEG}_{2000}$ to the side chains was intended to prevent nonspecific binding, yet some is still observed. Interestingly in the case of $\mathbf{C P N}_{\mathbf{P E}} \mathbf{E G}_{\mathbf{2 0 0}} \mathbf{- F A} / \mathrm{HA}$ complex, it is speculated that the polymers were internalized as large dense aggregates as seen in the contrast image (Figure 5.8f), indicating a strong non-covalent association between $\mathbf{C P N}_{\mathbf{P}} \mathbf{P E G _ { 2 0 0 0 }}$-FA and HA, with essentially no nonspecific binding observed after complexation. Moreover, the fluorescence intensity of CPN-PEG 2000-FA/HA complex increased significantly compared to the only $\mathbf{C P N}-\mathbf{P E G}$ 2000-FA, shown in the overlay image (Figure 5.6b). Similarly, a stronger more fluorescent signal was observed when cells were treated with CPN$\mathbf{P E G}_{2000} / \mathrm{HA}$ (Figure 5.8d) than CPN-PEG 2000 only (Figure 5.8c). While CPN-PEG 2000 displayed punctuated dots in the cytoplasm, $\mathrm{CPN}_{-} \mathrm{PEG}_{2000} / \mathrm{HA}$ shows punctuated dots as well as diffuse red signal throughout the cytoplasm, indicating a difference in uptake mechanism. These results emphasize and visually display the involvement of hyaluronan mediated endocytosis for significantly enhancing internalization of CPN/HA complexes.

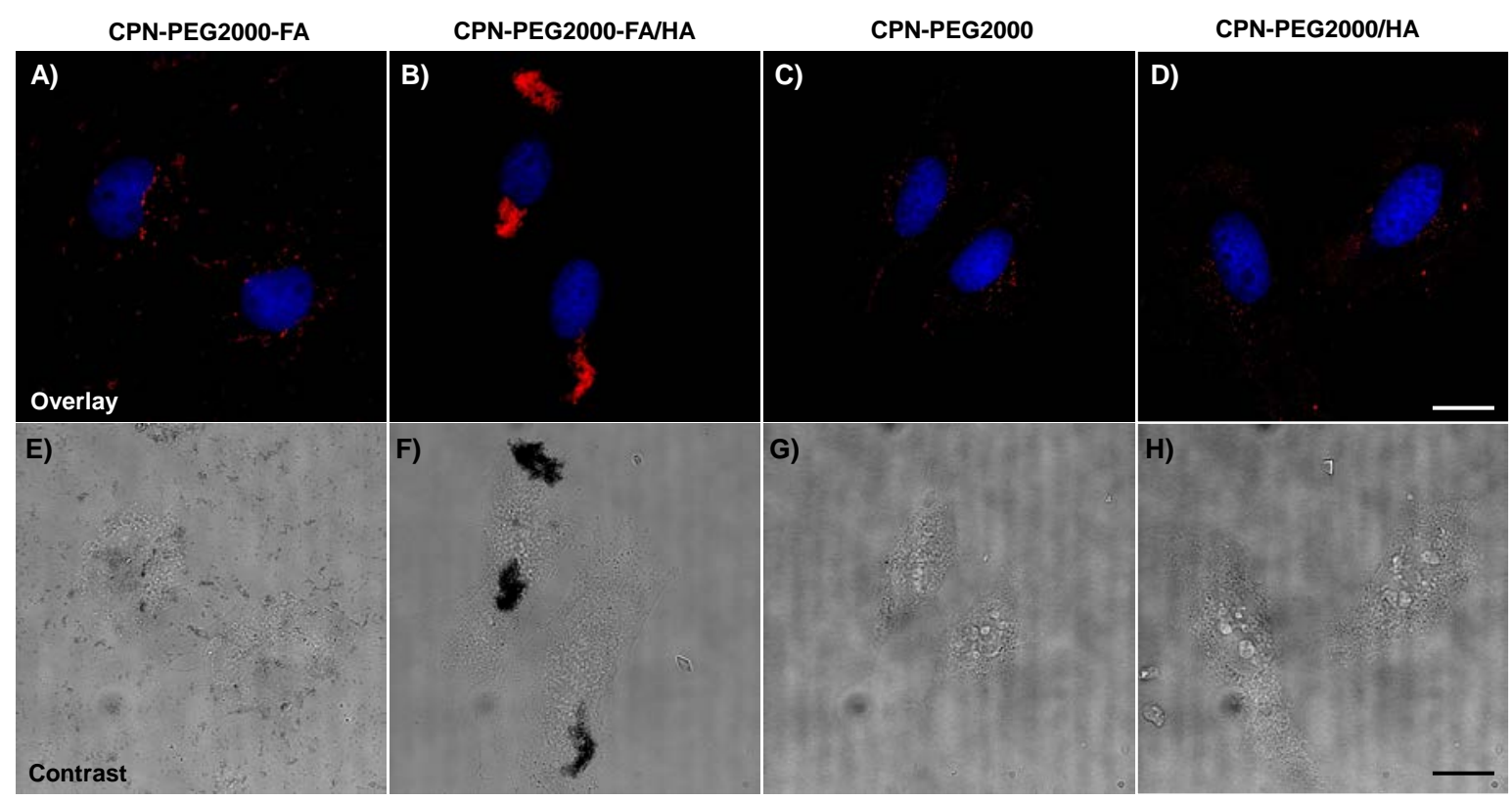

Figure 5.8. High-resolution fluorescence microscopic images (top row) of blue (nuclei) and red (CPNs) channel overlay, and contrast images (bottom row) of cellular uptake in HeLa cells for CPN-PEG 2000-FA (a and e), $\mathbf{C P N}_{\mathbf{P}} \mathbf{P E}_{2000}-\mathbf{F A} / \mathrm{HA}$ (b and f), $\mathbf{C P N}_{\mathbf{P}} \mathbf{P E G}_{2000}$ (c and g), and $\mathbf{C P N}_{\mathbf{P}} \mathbf{P G}_{2000} / \mathrm{HA}$ (d and h). Scale bar is $15 \mu \mathrm{m}$. 


\subsection{Conclusion}

In conclusion, highly fluorescent "dual-targeting” CPNs for bioimaging have been developed. The design utilizes simple synthetic strategies to modify the side chain of the conjugated polymer while conserving the emission of the polymer backbone in water. Specifically, ionic side chains and $\mathrm{PEG}_{2000}$ were incorporated into the polymer to create stable water-soluble nanomaterials. During PEG conjugation, the FA was selectively attached to the one end of PEG to ensure than they are available on the surface of the CPNs to bind with folate receptors of overexpressing cancer cells. The second ligand, HA, was incorporated as an electrostatic complex with CPNs via non-covalent interactions. High resolution fluorescence microscopy and inhibition studies confirmed that enhanced uptake of CPNs/HA complex was via receptor mediated

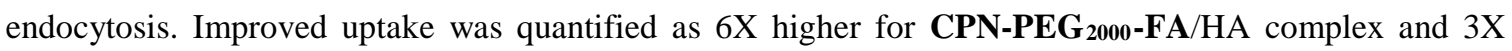
higher for CPN-PEG 2000/HA complex than CPNs only. Furthermore, these CPNs show minimal inherent toxicity toward HeLa cells. Therefore, the present study suggests that the dual ligand approach enhances the targeting efficiency of CPNs and could be very useful as nontoxic, biocompatible, highly fluorescent bioimaging agents. Utilization of these CPNs as far-red emissive imaging agents for sensitive animal study is underway. Moreover, incorporation of therapeutic agents in the side chain of the polymers will be investigated to develop water-soluble CPNs as a nontoxic and biodegradable delivery agent.

\subsection{Outlook}

This chapter presented a dual ligand approach to improve cellular interactions and uptake in cancer cells. The incorporation of the $\mathrm{PEG}_{2000}$ side chain provides increased solubility, but $\mathrm{PEG}_{2000}$-FA side chain poses a synthetic challenge to control the amount of incorporation as a result of the poor solubility of FA. An alternative method would be to modify the azide functionalized monomer side chain to increase hydrophilicity to then use a shorter ethylene oxide coupled to FA. By utilizing a shorter ethylene oxide chain, a clear understanding of uptake kinetics and pathways would provide useful information as to the role that folate and hyaluronan receptors play. Another alternative approach would be use microwave-assisted polymerization, which the Moon lab has recently started exploring. Under microwave irradiation, polymerization conditions are much more controlled and produce more reproducible results because of uniform heating and shorter reaction times. In order to accurately assess the dual ligand approach, a series of 
three CPNs are needed: CPN with PEG only side chain, CPN with PEG-FA side chain, and CPN with PEGFA complexed with HA. Since copolymers were used, a variation in co-polymer blocks was obtained based on synthetic approach. The first step would be to make an CPN array composed of non-BTZ doped PPEs with controlled incorporation of FA to accurately assess cellular interactions of FA and HA. Next, controlled incorporation of BTz units to the PPE backbone would provide a unique red emissive platform for improved sensitivity compared to green CPNs. A clear understanding of uptake pathways as function of the folate and hyaluronan receptors could be discerned from a defined CPN array. Subcellular localization studies of the dual targeting CPNs would provide information how modulating cell surface interactions affects subcellular trafficking and intracellular fate.

\subsection{Experimental}

All monomer and polymer synthesis/characterization and cell toxicity assay were completed Dr. Rajib Choudhury.

\subsubsection{Materials.}

Aluminium oxide (neutral, STD grade, 150 mesh, $50 \AA$ ), Dimethyl formamide (99.8\%), methyl sulfoxide $(99.0 \%+)$, dichloromethane $(99.8 \%+)$, hydroxybenzotriazole $($ HPLC, $99.0 \%+)$, folic acid $(97.0 \%+)$, 5hexynoic acid (97\%) and $\mathrm{N}$-(dimethylaminopropyl)- $\mathrm{N}^{\prime}$-ethylcarbodiimide hydrochloride were purchased from Sigma-Aldrich. Sodium chloride (laboratory grade), sodium azide (laboratory grade), silver oxide $(99.0 \%+)$, sodium iodide, sodium sulfate, magnesium sulfate, ammonium chloride, sulfuric acid, hydrochloric acid, ethyl acetate $\left(99 . \%^{+}\right)$, hexane, tetrahydrofuran $\left(99 . \%^{+}\right)$, N,N,N',N'tetramethylethylenediamine $(97 \%+), \mathrm{N}, \mathrm{N}^{\prime}$-dicyclohexylcarbodiimide $(99.0 \%+)$ and diethyl ether $(99.8 \%+)$ were purchased from Fisher Chemical. Celite-545, triethyl amine $(99.0 \%+)$, tetrahydrofuran $\left(99 . \%^{+}\right)$, copper iodide $(98 \%+)$, potassium iodide $(99.0 \%+)$, triphenyl phosphine $(99.0 \%+)$, $p$-toluenesulfonyl chloride $(99.0 \%+)$ 4-dimethylaminopyridine $\quad(99.0 \%+)$ diisopropylethyl $\quad$ amine $\quad(99.5 \%+) \quad$ and bis(triphenylphosphine)palladium (II) chloride (98\%) were purchased from Acros. Acetone and methyl alcohol (anhydrous $>99.9 \%$ ) were purchased from Marcon Fine Chemicals. PEG 2000 was obtained from Alfa Aesar. Copper bromide $(99.0 \%+)$ was obtained from Fluka. 4,7-dibromo-2,1,3-benzothiodiazole was purchased from Ark Pharm Inc. Carbon tetrabromide (99\%) and (trimethylsilyl)acetylene (99\%) were 
purchased from Oakwood Chemical. Silica gel $(40-63 \mu \mathrm{m})$ was obtained from Silicycle Inc. Thin layer chromatography plates (TLC silica gel 60 F254) was obtained from Merck. All deuterated solvents were purchased from Cambridge Iosotope Laboratories, Inc (Cambridge, MA). $\mathrm{PEG}_{2000}$ was freeze dried before use. $\mathrm{N}, \mathrm{N}^{\prime}$-dicyclohexylcarbodiimide was recrystallized from chloroform and dried overnight under high vacuum before use. All chemicals were used without further purification unless stated otherwise.

All biological reagents were used without further purification. HeLa cells (human cervical cancer) was purchased from ATCC. 100 x 20 mm style sterile tissue culture dish (\#353003 BD Falcon, Durham, NC, USA), 96-well plate (\#3795 Costar clear polystyrene 96-well plates, Corning Life Sciences Plastic), 12-well plate (\#130185 BioLite 12-well plates, Thermo Scientific), minimum essential medium (MEM)/Earle’s balanced salt solution (EBSS, HyClone, SH30024), fetal bovine serum (FBS, Hyclone FetalClone I serum, SH3008002, GE Healthcare), $100 \mathrm{U} / \mathrm{mL}$ penicillin and $100 \mu \mathrm{g} / \mathrm{mL}$ streptomycin (Penicillin/Streptomycin Mixture, Fisher BioReagents, BP2959-50), and MTT(CALBIOCHEM, Germany) were purchased from Fisher Scientific. Sodium hyaluronate (HA) was purchased from Lifecore (MW 40K and $100 \mathrm{~K}$ ) and used as received. Stock solutions were prepared by dissolving $2.3 \mathrm{mg}$ and $2.2 \mathrm{mg}$ of $40 \mathrm{~K}$ and $100 \mathrm{~K} \mathrm{HA}$, respectively, in $1 \mathrm{~mL}$ deionized water. Pharmacological endocytosis inhibitors such as chlorpromazine hydrochloride (Assay Designs, Inc), genistein (Acros Organics), methyl- $\beta$-cyclodextrin (Acros Organics), LY2994002 (Caymen Chemical), and cytochalasin D (Assay Designs, Inc) were purchased from Fisher Scientific. Trypan blue was purchased from Amresco, Inc. and used as received.

\subsubsection{Instrumentation.}

Nuclear magnetic resonance (NMR) data were recorded on a Bruker Avance 400 spectrometer operating at $400 \mathrm{MHz}$ for ${ }^{1} \mathrm{H}$ NMR and $100.6 \mathrm{MHz}$ for ${ }^{13} \mathrm{C}$ NMR at $25{ }^{\circ} \mathrm{C}$. The acquired data was processed in the TOPSPIN 3.1 software. Chemical shifts are represented in parts per million (ppm) and referenced to $\mathrm{CDCl}_{3}$ $(\delta \sim 7.26 \mathrm{ppm})$, DMSO $(\delta \sim 2.50 \mathrm{ppm})$ solvent peak. The spectra were recorded with a spectral width (SW) of 8 ppm and 64 k data points. UV-vis data were collected on a Varian Cary 50 Bio spectrometer. Fluorescence spectra were obtained using a FluoLog-3 Spectrofluorometer (Jobin Yvon/Horiba). Quantum yields were calculated using Coumarin $6(\mathrm{QY}=0.6)$ in ethyl alcohol as a fluorescence standard. Graphs were plotted using Origin 9.1 software (OriginLab, Northampton, MA, USA). Fourier transform infrared (FT-IR) spectra 
were recorded on a PerkinElmer Spectrum 100 FT-IR Spectrometer. For each sample, 16 spectra were collected at a $4 \mathrm{~cm}^{-1}$ spectral resolution and with a spectral width ranging from $4000 \mathrm{~cm}^{-1}$ to $700 \mathrm{~cm}^{-1}$. Background subtraction and pick picking were performed in the OMNIC software. High-resolution mass spectrometry data were recorded on a Bruker Daltonics ultrOTOF Q mass spectrometer with electron ionization mode.

\subsubsection{Monomer design.}

Monomers were synthesized according to procedures described in literature and synthetic routes for monomers M1 and M2 are shown Scheme S5.1. Alkoxy side chains were incorporated in the aromatic rings to enhance solubility of the monomers and corresponding conjugated polymers in organic solvents. Moreover, to enhance aqueous solubility of the resulting polymer nanoparticles (NPs), bromo groups were incorporated in the terminal position of the alkoxy side chain of $\mathbf{M 1}$ which was replaced in situ by triethyl amine during Sonogashira polymerization at elevated temperature, resulting in a water soluble quaternary ammonium ion.

Compound 3 (1,4-bis(2-(2-(2-bromoethoxy)ethoxy)ethoxy)-2,5-diiodobenzene) was prepared from readily available compound $\mathbf{2}$. Compound $\mathbf{4}$ was obtained via Sonogashira reaction of $\mathbf{3}$ with trimethylsilyl acetylene, which was then stirred in the solution of methanol and potassium carbonate to afford final M1 in 58\% yield. Presence of two terminal alkyne protons was confirmed by comparing the integration ratio of signal at 3.34 ppm with aromatic signals at 7.00 ppm (see Figure S1 for full NMR spectra). Compound 6 was prepared from 5 via Appel reaction, then the terminal bromides were converted into azides by reaction with sodium azide in DMF at $70^{\circ} \mathrm{C}$ for 6 hours. ${ }^{1} \mathrm{H}-\mathrm{NMR}$ and ${ }^{13} \mathrm{C}$ NMR spectroscopy confirmed that the conversion of the terminal bromides into azides was quantitative (see figure S2, S3 for ${ }^{1} \mathrm{H}$ and ${ }^{13} \mathrm{C}$ NMR of M2). The product was also characterized by IR spectroscopy. The typical band at $\sim 2100 \mathrm{~cm}^{-1}$ owing to the conversion of the bromide termini into azide group appeared as expected (Figure S4).

M3 (FA-PEG 2000 -propargyl) was prepared using a modified procedure reported by Mahou et.al. (as shown in Scheme S5.1). ${ }^{25}$ First, mono-tosyl PEG $_{2000}$ was synthesized with $90 \%$ yield. Subsequently, the tosyl end group was converted into azide and then into amine group. ${ }^{1} \mathrm{H}$ NMR spectroscopy confirmed that conversion of azide to amine was quantitative. The amine end group of compound $\mathbf{9}$ was then successfully coupled to 
hex-5-ynoic acid to afford 10. Finally, M3 was prepared from DCC/DMAP coupling reaction of FA with 10, as a 1:1 mixture of $\mathbf{1 0}$ and $\mathbf{M 3}$. The mixture was used without further purification for grafting of conjugated polymers.
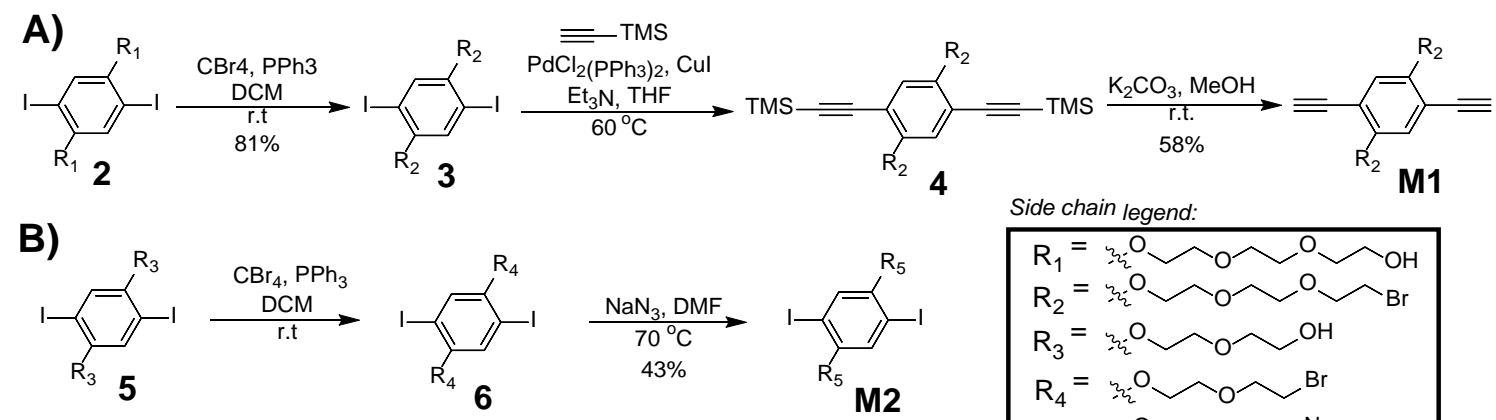

C)
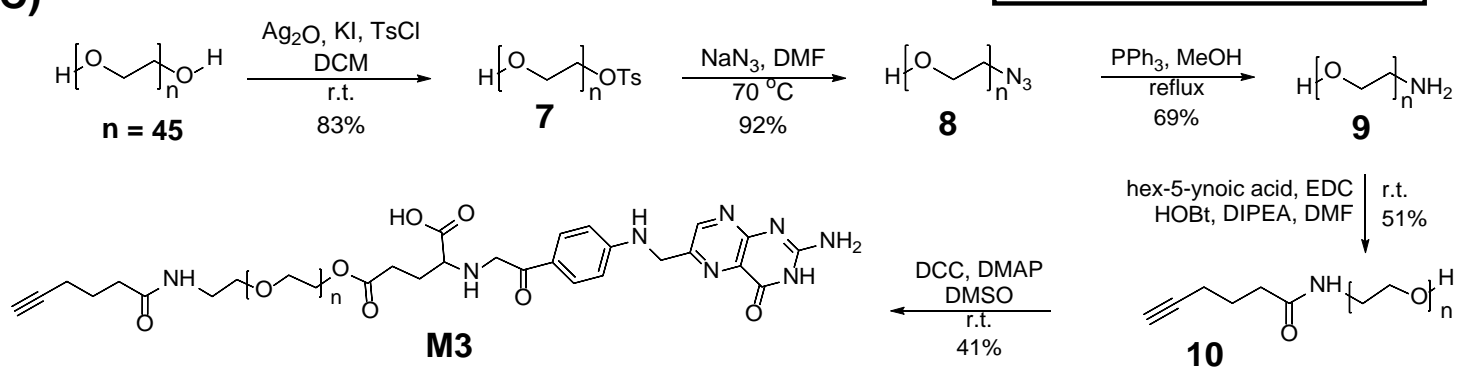

Scheme S5.1. Synthesis of monomers (M1, M2) and heterodifunctional PEG 2000 (M3).

\subsubsection{Monomer synthesis.}

Synthesis of 1,4-bis(2-(2-(2-bromoethoxy)ethoxy)ethoxy)-2,5-diethynylbenzene (M1). Following literature procedure, synthesis of $\mathbf{M 1}$ was carried out in three steps starting from readily available compound 2. Briefly, Carbon tetrabromide (8.0 g, $23.95 \mathrm{mmol})$ and triphenyl phosphine (12.60 g, $23.95 \mathrm{mmol})$ were added to a stirred solution of 2 in DCM under nitrogen atmosphere. The resulting solution was stirred for 2 h at room temperature. After the solvent was removed the residue was purified by silica gel chromatography (ethyl acetate/hexane 1:4) to give a white solid compound (3). Yield (5.3 g, 81.2\%). ${ }^{1} \mathrm{H} \mathrm{NMR}\left(400 \mathrm{MHz}, \mathrm{CDCl}_{3}\right)$ $\delta 7.26(\mathrm{~s}, 2 \mathrm{H}), 4.14(\mathrm{t}, 4 \mathrm{H}), 3.91(\mathrm{t}, 4 \mathrm{H}), 3.85(\mathrm{t}, 4 \mathrm{H}), 3.81(\mathrm{t}, 4 \mathrm{H}), 3.73(\mathrm{t}, 4 \mathrm{H}), 3.50(\mathrm{t}, 4 \mathrm{H}) .{ }^{13} \mathrm{C}$ NMR $(100.6$ $\left.\mathrm{MHz}, \mathrm{CDCl}_{3}\right) \delta 153.4,123.5,86.5,71.3,71.2,70.7,70.3,69.7,30.4$.

Under nitrogen atmosphere, $\mathrm{Pd}\left(\mathrm{PPh}_{3}\right)_{2} \mathrm{Cl}_{2}(0.23 \mathrm{~g}, 0.33 \mathrm{mmol})$ and $\mathrm{CuI}(0.031 \mathrm{~g}, 0.16 \mathrm{mmol})$ were added to a solution of 3 (2.24 $\mathrm{g}, 3.3 \mathrm{mmol})$ in $30 \mathrm{~mL}$ of anhydrous THF and trimethylamine mixture (2:1). Trimethylsilylacetylene $(0.97 \mathrm{~g}, 9.8 \mathrm{mmol})$ was slowly added to the mixture at room temperature. The 
reaction mixture was then refluxed under nitrogen for $3 \mathrm{~h}$. The solvent was removed under reduced pressure. The residue was passed through a short column of silica gel with hexane as the eluent. The evaporation of the solvent led to a yellow solid which was used in next step without further purification. In a $100 \mathrm{~mL}$ round bottom flask, 4 (4.38 g, $6.0 \mathrm{mmol}), \mathrm{K}_{2} \mathrm{CO}_{3}(1.65 \mathrm{~g}, 12 \mathrm{mmol})$ were added in $30 \mathrm{~mL} \mathrm{MeOH}$. The resulting slurry was stirred for $3 \mathrm{~h}$. The progression of the reaction was monitored by thin layer chromatography. After $4 \mathrm{~h}$, solvent was evaporated to dryness and purified by silica gel column chromatography (hexane as eluent). Yield (2.0 g, 58\%). ${ }^{1} \mathrm{H}$ NMR (400 MHz, $\left.\mathrm{CDCl}_{3}\right) \delta 7.00$ (s, 2H), 4.15 (t, 4H), 3.87 (t, 4H), 3.81 (t, 4H), 3.78 (t, 4H), 3.76 (t, 4H), 3.69 (t, 4H), 3.47 (t, 4H), 3.33 (s, 2H). ${ }^{13} \mathrm{C}$ NMR (100.6 MHz, $\left.\mathrm{CDCl}_{3}\right) \delta$ 154.1, 118.5, 113.6, 82.7, 79.6, 71.3, 71.1, 70.7, 69.7, 69.6, 30.3. HRMS (ESI) m/z calcd. for $\left[\mathrm{C}_{22} \mathrm{H}_{28} \mathrm{Br}_{2} \mathrm{O}_{6}\right]$ 548.0232; found for $\left[\mathrm{C}_{22} \mathrm{H}_{28} \mathrm{Br}_{2} \mathrm{O}_{6}+\mathrm{H}\right]^{+} 549.0295$.

Synthesis of 1,4-bis(2-(2-azidoethoxy)ethoxy)-2,5-diiodobenzene (M2). This compound was synthesized according to procedures described in literature. The product was white crystals (0.5g, 43\%). ${ }^{1} \mathrm{H}$ NMR (400 $\left.\mathrm{MHz}, \mathrm{CDCl}_{3}\right) \delta 7.24(\mathrm{~s}, 2 \mathrm{H}), 4.10(\mathrm{t}, 4 \mathrm{H}), 3.88(\mathrm{t}, 4 \mathrm{H}), 3.79(\mathrm{t}, 4 \mathrm{H}), 3.69(\mathrm{t}, 4 \mathrm{H}), 3.39(\mathrm{t}, 4 \mathrm{H}) .{ }^{13} \mathrm{C}$ NMR $\left(100.6 \mathrm{MHz}, \mathrm{CDCl}_{3}\right) \delta 153.2,123.5,86.5,71.3,71.2,70.8,70.3,70.1,69.7,50.7$.

Synthesis of $\alpha$-tosyl- $\omega$-hydroxyl PEG 2000 (7). PEG (2000 g/mol, 20.0 g, 0.01 mol), previously freeze-dried, was dissolved in $250 \mathrm{~mL}$ of anhydrous DCM. Ag $2 \mathrm{O}$ (1.5 eqv., $3.55 \mathrm{~g}, 0.015 \mathrm{~mol}$ ) and $\mathrm{KI}$ (0.2 eqv., $0.332 \mathrm{~g}$, $0.002 \mathrm{~mol}$ ) were added. To this rapidly stirred solution, $\mathrm{TsCl}$ (1.05 eqv., $2.0 \mathrm{~g}, 0.0105 \mathrm{~mol}$ ) was added in one portion. The reaction mixture was stirred for $18 \mathrm{~h}$ at room temperature before filtration over a filter cell cake and solvent removal by rotary evaporation was performed. The crude product was dissolved in $30 \mathrm{~mL}$ DCM and then precipitated by dropwise addition into diethyl ether (300 mL, 3x). The white hygroscopic compound was collected by vacuum filtration under nitrogen atmosphere and dried overnight under high vacuum. Yield (18 g, 83\%). ${ }^{1} \mathrm{H}$ NMR (400 MHz, DMSO-d 6 ) $\delta 7.78$ (d, 2H), 7.48 (d, 2H), 4.56 (t, 1H), $4.11(\mathrm{~d}, 2 \mathrm{H}), 3.50$ (br, 191H), 2.42 (s, 3H). ${ }^{13} \mathrm{C}$ NMR (100.6 MHz, DMSO-d 6 ) $\delta$ 144.9, 132.4, 130.1, 127.6, 72.4, 69.9, 69.8, 67.9, 60.2, 21.0. IR (neat) v 3454, 2882, 2865, 1464, 1339, 1098, $1055 \mathrm{~cm}^{-1}$.

Synthesis of $\alpha$-azide- $\omega$-hydroxyl PEG 2000 (8). In a $250 \mathrm{~mL}$ round-bottom flask $\alpha$-tosyl- $\omega$-hydroxyl PEG (7, $10 \mathrm{~g}, 0.0046 \mathrm{~mol}$ ) was dissolved in $50 \mathrm{~mL}$ dry DMF. Then $\mathrm{NaN}_{3}$ (5.0 eqv., $1.5 \mathrm{~g}, 0.023 \mathrm{~mol}$ ) was added in 
one portion. The reaction mixture was stirred overnight at $70{ }^{\circ} \mathrm{C}$ under nitrogen atmosphere. After cooling down to room temperature and filtration, DMF was removed under vacuum. Excess $\mathrm{NaN}_{3}$ was quenched by addition of water. The crude product was dissolved in $200 \mathrm{~mL}$ DCM and washed thrice with brine and twice with water. The organic layer was dried over sodium sulfate, reduced to a small volume by rotary evaporation, and finally precipitated by dropwise addition into diethyl ether $(250 \mathrm{~mL}, 3 \mathrm{x})$. The white precipitate was collected by vacuum filtration and dried overnight under high vacuum. Yield (8.7 g, 92\%). ${ }^{1} \mathrm{H}$ NMR (400 MHz, DMSO-d 6 ) $\delta 4.57(\mathrm{br}, 1 \mathrm{H}), 3.68(\mathrm{t}, 2 \mathrm{H}), 3.50(\mathrm{br}, 206 \mathrm{H}) .{ }^{13} \mathrm{C}$ NMR (100.6 MHz, DMSO-d 6 ) $\delta 72.3$, 69.8, 69.2, 60.2, 49.9. IR (neat) v 3454, 2882, 2865, 2108, 1464, 1343, 1274, 1103, $1055 \mathrm{~cm}^{-1}$.

Synthesis of $\alpha$-amine- $\omega$-hydroxyl PEG 2000 (9). $\alpha$-azide- $\omega$-hydroxyl PEG 2000 (8, 4.0 g, 0.00195 mol) and $\mathrm{PPh}_{3}$ (3.0 eqv., $1.53 \mathrm{mg}, 0.00585 \mathrm{~mol}$ ) were dissolved in $30 \mathrm{~mL} \mathrm{MeOH}$. The resulting mixture was refluxed overnight under nitrogen atmosphere at $70{ }^{\circ} \mathrm{C}$ and then cooled down to room temperature. After solvent removal by rotary evaporation, the crude mixture was dissolved in $20 \mathrm{~mL}$ DCM, washed twice with brine and dried over sodium sulfate. Solvent was evaporated to dryness, dissolved in $5 \mathrm{~mL}$ DCM and precipitated in diethyl ether (200 mL, 3x). White powder was collected by vacuum filtration and dried overnight under high vacuum. Yield (2.7 g, 69\%). ${ }^{1} \mathrm{H}$ NMR (400 MHz, DMSO-d 6 ) $\delta 4.58(\mathrm{br}, 1 \mathrm{H}), 3.51$ (br, 204H), $2.64(\mathrm{t}$, 2H). ${ }^{13} \mathrm{C}$ NMR (100.6 MHz, DMSO-d 6 ) $\delta$ 72.3, 69.8, 60.2. IR (neat) v 3454, 2879, 1466, 1344, 1276, 1098 $\mathrm{cm}^{-1}$.

Synthesis of $\alpha$-(hex-5-ynamide)- $\omega$-hydroxyl PEG 2000 (10). $\alpha$-amine- $\omega$-hydroxyl PEG P $_{2000}$ (9, 4.63g, 0.0023 mol), 5-hexynoic acid (0.283 g, $0.0025 \mathrm{~mol}$ ) were dissolved in $10 \mathrm{~mL}$ anhydrous DMF, followed by addition of EDC (1.2 eqv., 0.431 g, 0.00278 mol), HOBt (1.2 eqv., 0.375 g, 0.00278 mol) and DIPEA (1.2 eqv., 0.359 g, $0.00278 \mathrm{~mol})$. The reaction mixture was stirred for $24 \mathrm{~h}$ under nitrogen atmosphere at room temperature and then filtered to remove insoluble byproducts. The filtrate was concentrated under high vacuum and precipitated in diethyl ether $(100 \mathrm{ml})$. Solid precipitate was collected under vacuum filtration, dissolved in $20 \mathrm{~mL}$ DCM, washed thrice with deionized water and then dried over sodium sulfate. Solvent was evaporated under rotary evaporation, precipitated in diethyl ether $(100 \mathrm{~mL}, 2 \mathrm{x})$ and collected under vacuum filtration. Yield (2.5 g, 51\%). ${ }^{1} \mathrm{H}$ NMR (400 MHz, DMSO-d 6 ) $\delta 7.89$ (t, 2H), 4.59 (t, 2H), 3.51 (br, 200H), 2.77 (s, 
1H), 2.12-2.18 (m, 4H), 1.61-1.68 (m, 2H). ${ }^{13} \mathrm{C}$ NMR (100.6 MHz, DMSO-d 6 ) $\delta$ 171.7, 84.1, 72.3, 71.3, 69.7, 60.1, 34.4, 24.3, 17.2. IR (neat) v 3424, 3274, 2887, 1651, 1467, 1340, $1103 \mathrm{~cm}^{-1}$.

Synthesis of heterodifunctional $\alpha$-(hex-5-ynamide)- $\omega$-folic acid PEG 2000 (M3). Folic acid (92.74 mg, 0.210 mmol), DCC (43.32 mg, $0.210 \mathrm{mmol}$ ) were dissolved in $10 \mathrm{ml}$ anhydrous DMSO, under nitrogen atmosphere, followed by addition of DMAP (10 mol\%, $2.56 \mathrm{mg}$ ) and $\alpha$-(hex-5-ynamide)- $\omega$-hydroxyl PEG 2000 (10, 400 $\mathrm{mg}, 0.191 \mathrm{mmol}$ ). The reaction mixture was stirred in the dark at room temperature for $24 \mathrm{~h}$ and then centrifuged to remove the insoluble byproduct, dicyclohexylurea. The supernatant was concentrated in vacuo to $2 \mathrm{~mL}$ and precipitated into $50 \mathrm{~mL}$ DCM. The insoluble, unreacted folic acid was removed via vacuum filtration and the filtrate concentrated in vacuo. The resulting residue was dissolved in $2 \mathrm{~mL}$ DCM and precipitated in $50 \mathrm{~mL}$ diethyl ether. The step was repeated two more times. The precipitated product was collected via centrifugation and dried overnight under high vacuum. ${ }^{1} \mathrm{H}$ NMR signal integration was used to determine the conjugation efficiency (50\%) and the mixture was not separated. Yield (200 mg, 41\%). ${ }^{1} \mathrm{H}$ NMR (400 MHz, DMSO-d 6 ) $\delta 11.49(\mathrm{~s}, 1 \mathrm{H}), 8.65$ (d, 1H), 8.19 (d, 1H), 7.88 (t, 1H), 7.65 (d, 2H), 7.45 (d, 2H), 6.93 (d, 1H), $6.62(\mathrm{~m}, 2 \mathrm{H}), 4.57$ (t, 2H), 4.48 (t, 1H), 3.52 (br, 219H), 2.76 (s, 1H), 2.31 (t, 1H), 2.15 (m, 4H), 1.63 (m, 2H). IR (neat) v 3500-2500 (br), 1692, 1601, 1510, 1300, $1205 \mathrm{~cm}^{-1}$.

\subsubsection{Polymer synthesis.}

Conjugated polymers PPE1 and PPE2 were obtained by Sonogashira cross-coupling polymerization between monomers $\mathbf{M} 1$ and $\mathbf{M} 2$ and commercially available monomer 4,7dibromobenzo[c][1,2,5]thiadiazole in yield of 50 60\% as red solids (Scheme S5.2 and S5.3). Both polymers are partially soluble in boiling THF and chloroform but fully soluble in DMF, DMSO, MeOH, and $\mathrm{H}_{2} \mathrm{O}$. Upon drying they strongly aggregate and do not redissolve in $\mathrm{H}_{2} \mathrm{O}$ but solubility in DMSO and DMF remains unaffected. Minimal solubility in THF and cationic side chains of the polymers precluded the measurement of their molecular weight by gel permeation chromatography (GPC).

\subsubsection{Synthesis of PPE1.}

A Schlenk flask was loaded with 1,4-bis(2-(2-(2-bromoethoxy)ethoxy)ethoxy)-2,5-diethynylbenzene (M1, $26.4 \mathrm{mg}, 0.048 \mathrm{mmol})$, 1,4-bis(2-(2-azidoethoxy)ethoxy)-2,5-diiodobenzene (M2, $14.15 \mathrm{mg}, 0.024 \mathrm{mmol}$ ), 4,7-dibromobenzo[c][1,2,5]thiadiazole (7.07 mg, $0.024 \mathrm{mmol}), \mathrm{Pd}\left(\mathrm{PPh}_{3}\right)_{2} \mathrm{Cl}_{2}(1.69 \mathrm{mg}, 0.0024 \mathrm{mmol})$ and 
CuI (0.228 mg, $0.0012 \mathrm{mmol})$. The flask was degassed and back-filled with nitrogen for 2 minutes and $4 \mathrm{~mL}$ of air-free solvent $\left(\mathrm{Et}_{3} \mathrm{~N}\right.$ : DMF; 2:1) was added via a cannula. The mixture was heated at $65{ }^{\circ} \mathrm{C}$ for $12 \mathrm{~h}$ under nitrogen atmosphere. The solution was then cooled to room temperature and transferred dropwise to ethyl acetate, resulting in precipitation. Precipitate was collected via centrifugation (3 mins., 4000 rpm), dissolved in $1 \mathrm{~mL}$ DMF and mixed with $10 \mathrm{~mL} \mathrm{MeOH}$. The resulting viscous solution was filtered through PTFE syringe filter (cut-off $0.45 \mu \mathrm{m}$ ). Filtrate was evaporated to low volume ( 0.5 mL) in vacuo, precipitated in $40 \mathrm{~mL}$ ethyl acetate (3x). The supernatant was discarded and the solid red precipitated was collected, dried under high vacuum for $12 \mathrm{~h}$ prior to ${ }^{1} \mathrm{H}$ NMR and FTIR characterization. Yield (39 mg, 50\%). ${ }^{1} \mathrm{H}$ NMR (400 MHz, DMSO-d $\left.{ }_{6}\right) \delta 7.99$ (br m, 0.5 H), 7.34 (br, 1H), 7.20 (br, 2H), 4.24 (br, 6H), 3.86-3.39 (br, 26H), 3.28 (br, 12H), 1.13 (br, 18H). IR (neat) v 3411, 2926, 2870, 2100, 1492, 1463, 1417, 1275, 1216, $1117 \mathrm{~cm}^{-1}$. UVVis (THF) $\lambda_{\max }=452 \mathrm{~nm}, \varepsilon=59171.59 \mathrm{M}^{-1} \mathrm{~cm}^{-1}$ (per repeating unit), emission (THF) $\lambda_{\max }=578 \mathrm{~nm}, \mathrm{Q} . Y .=$ 0.31 (in THF).

\subsubsection{Synthesis of CPN-PEG $2000-F A$.}

Heterodifunctional $\alpha$-(hex-5-ynamide)- $\omega$-folic acid PEG 2000 grafted conjugated polymer (CPN-PEG 2000-

FA) was synthesized by click reaction. PPE1 (7.0 mg, $0.0076 \mathrm{mmol}$ ) and M3 (29.2 mg, $0.0114 \mathrm{mmol}$ ) were dissolved in $4 \mathrm{~mL}$ of anhydrous DMF in a flame-dried and nitrogen-purged vial. Then, $\mathrm{CuBr}$ ( $0.33 \mathrm{mg}, 0.0023$ mmol) and TEMED (0.267 mg, $0.0023 \mathrm{mmol})$ were added sequentially. The vial was degassed and backfilled with nitrogen for 2 minutes and stirred for $48 \mathrm{~h}$ at $30{ }^{\circ} \mathrm{C}$. The reaction mixture was exposed to air and passed through a short column of alumina to remove unreacted $\mathrm{CuBr}$. The crude polymer was obtained by precipitation into diethyl ether. Then it was dissolved in $1 \mathrm{~mL}$ of DMSO, added into $10 \mathrm{~mL}$ deionized water and washed with $10 \mathrm{~mL}$ DCM (10x). The aqueous fraction was collected, dialyzed (dialysis membrane, MWCO: 5000) with 2 L deionized water to remove DMSO and unreacted PEG 2000 . The pure polymer was recovered by lyophilization. ${ }^{1} \mathrm{H}$ NMR (400 MHz, DMSO-d $\left.{ }_{6}\right) \delta 8.02(\mathrm{br}, 0.7 \mathrm{H}), 7.88$ (br, 0.3H), 7.79 (br, 0.7H), 7.56 (br, 0.54H), 7.5-7.1 (br, m, 4.2H), 4.64 (br, 0.78H),4.24 (br, 6H), 4.0-3.52 (br, m, 23H), 3.51 (br, 68H), 3.28 (br, 12H), 2.15 (br, m, 1.6H), 1.65 (br, m, 0.72H), 1.13 (br, 18H). IR (neat) v 3600-2500 (br), 2104, 1642, 1603, 1492, 1454, 1342, 1275, $1101 \mathrm{~cm}^{-1}$. UV-Vis $\left(\mathrm{H}_{2} \mathrm{O}\right) \lambda_{\max }=481 \mathrm{~nm}$, emission $\left(\mathrm{H}_{2} \mathrm{O}\right) \lambda_{\max }$ $=618 \mathrm{~nm}, \mathrm{Q} . \mathrm{Y} .=0.55$ (in water). 


\subsubsection{Characterization of PPE1 and CPN-PEG $2000-F A$.}

Comparison between the integrated areas for the methyl groups of quaternary ammonium ion at $1.13 \mathrm{ppm}$ and methylene hydrogens at $3.28 \mathrm{ppm}$ to that of aryl hydrogens at 7.20-7.34 ppm and hydrogens on ethoxy chains at $~ 3.59-4.24$ ppm in the ${ }^{1} \mathrm{H}$ NMR spectrum of PPE1 reveals that 50\% of M1 has been incorporated as quaternary ammonium ions in the side chain. Similarly, integration comparison between the aromatic signals in BTz and all other signals from polymeric backbone, as well as side chains, suggest 25\% incorporation of BTz units in the polymer.

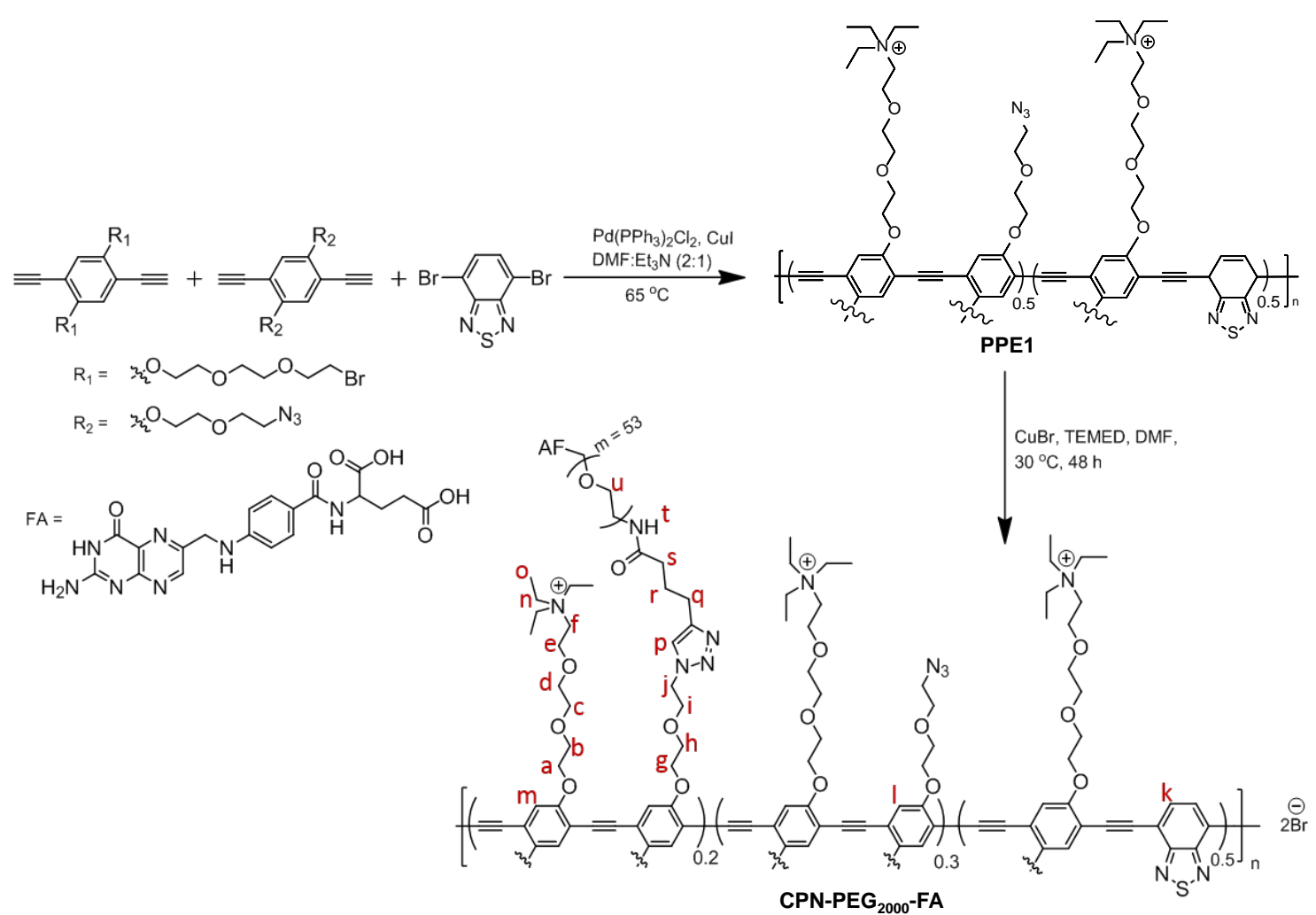

Scheme S5.2. Synthesis of water-soluble PPE1 from readily available monomers via Sonogashira coupling and grafting to yield $\mathbf{C P N}-\mathbf{P E G}_{2000}-\mathbf{F A}$.

The quantitative character of the 'click' reaction was determined by ${ }^{1} \mathrm{H}-\mathrm{NMR}$ in DMSO- $\mathrm{d}_{6}$. Figure S5.1 shows the comparison of ${ }^{1} \mathrm{H}-\mathrm{NMR}$ spectra of PPE1 (top spectra) with the final PEG $2000-\mathrm{FA}$ grafted copolymer (CPN-PEG 2000-FA, bottom spectra) obtained after the dipolar cycloaddition with PEG $2000-\mathrm{FA}$. New signal at $3.51 \mathrm{ppm}$ is assigned to the repeat unites $-\mathrm{CH}_{2}-\mathrm{CH}_{2}-\mathrm{O}-$ from $\mathrm{PEG}_{2000}$, while signals at 1.65 ppm and $2.15 \mathrm{ppm}$ are assigned to methylene protons $\mathbf{r}$ and $\mathbf{q}+\mathbf{s}$, respectively. The peak at $4.64 \mathrm{ppm}$ is related to the protons from FA. Moreover, signals at $\delta$ 7.20-7.56, 7.79 and 8.01 are assigned to FA, where signal at 
$7.88 \mathrm{ppm}$ is characteristic to amide signal of the PEG derivative. Also, NMR signal integration indicates presence of proton from triazole linkage buried at $\delta$ 7.20-7.56 ppm. Similarly, the ratio of proton integrals belonging to PPE1 to that of $\mathrm{PEG}_{2000}$-FA suggests $40 \%$ incorporation of the latter via click reaction.
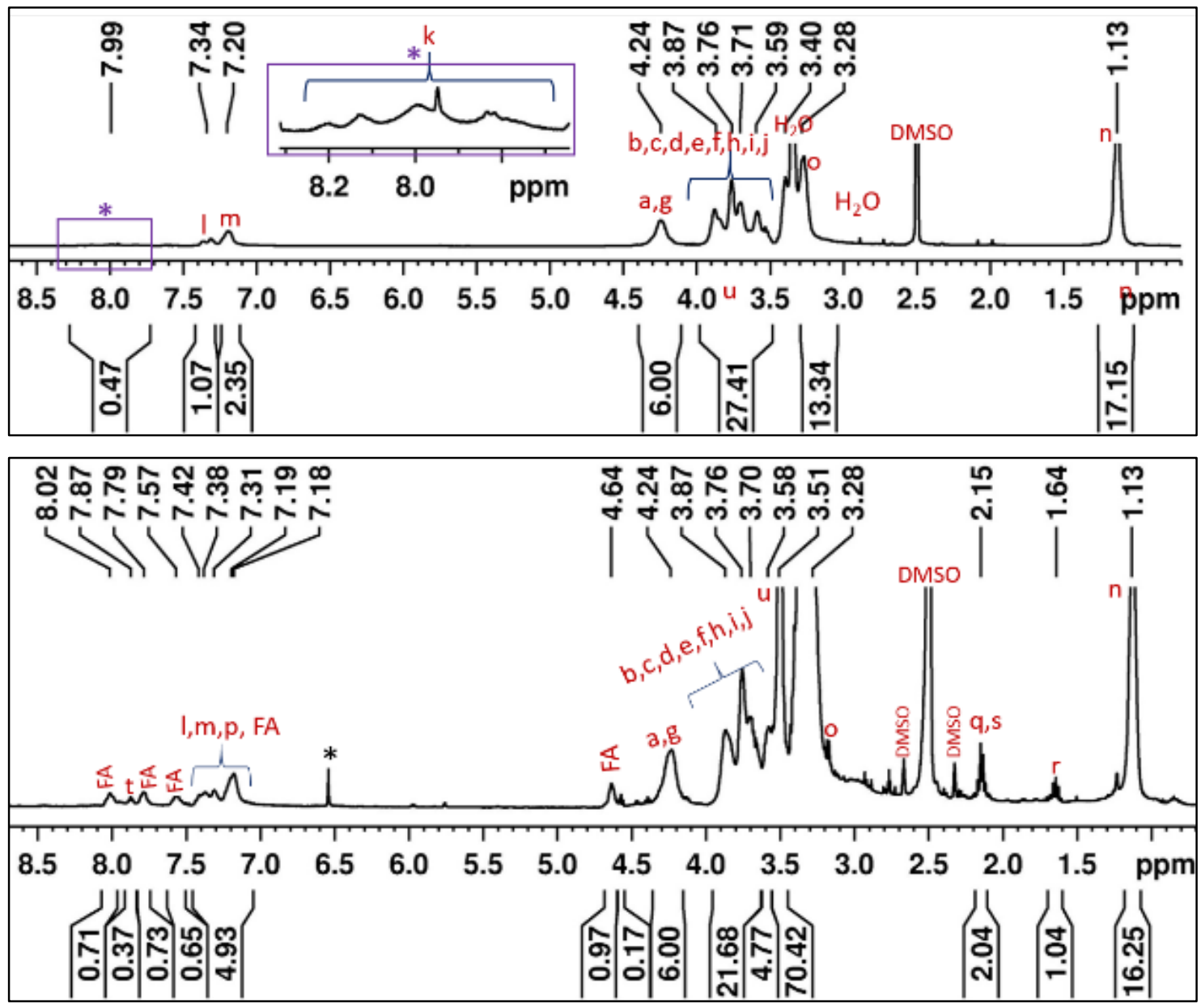

Figure S5.1. ${ }^{1} \mathrm{H}$ NMR spectra of PPE1 (top) and CPN-PEG $2000-F A$ (bottom) in DMSO-d $6 .{ }^{*}$ indicates unassigned signal.

To prepare the grafted copolymers $\mathbf{C P N}_{\mathbf{P E}} \mathbf{2 0 0 0}_{\mathbf{2 0}} \mathbf{F A}$ and $\mathbf{C P N}_{\mathbf{P}} \mathbf{P E G}_{\mathbf{2 0 0 0}}$, the azide functionalized PPEs were coupled with alkynyl end of $\mathrm{PEG}_{2000}-\mathrm{FA}$ and $\mathrm{PEG}_{2000}$, respectively, by the Huisgen 1,3-dipolar cycloaddition. For CPN-PEG 2000-FA, “click reaction” between PPE1 and M3 in presence of catalytic amount of TEMED and CuBr in DMF at room temperature afforded CPN-PEG 2000 $_{2}$-FA in 45\% yield. An excess of alkynyl-terminated PEG 2000 -FA (2.0 equivalent) was used in order to increase the grafting efficiency. The crude copolymer was first washed with excess DCM (10x) to remove unreacted PEG 2000 and 
other organic impurities followed by dialysis ( $5 \mathrm{kDa}$ molecular weight cut-off dialysis membrane). Complete removal of free FA was confirmed by monitoring absorption of FA in the aqueous supernatant (Figure S5.2).

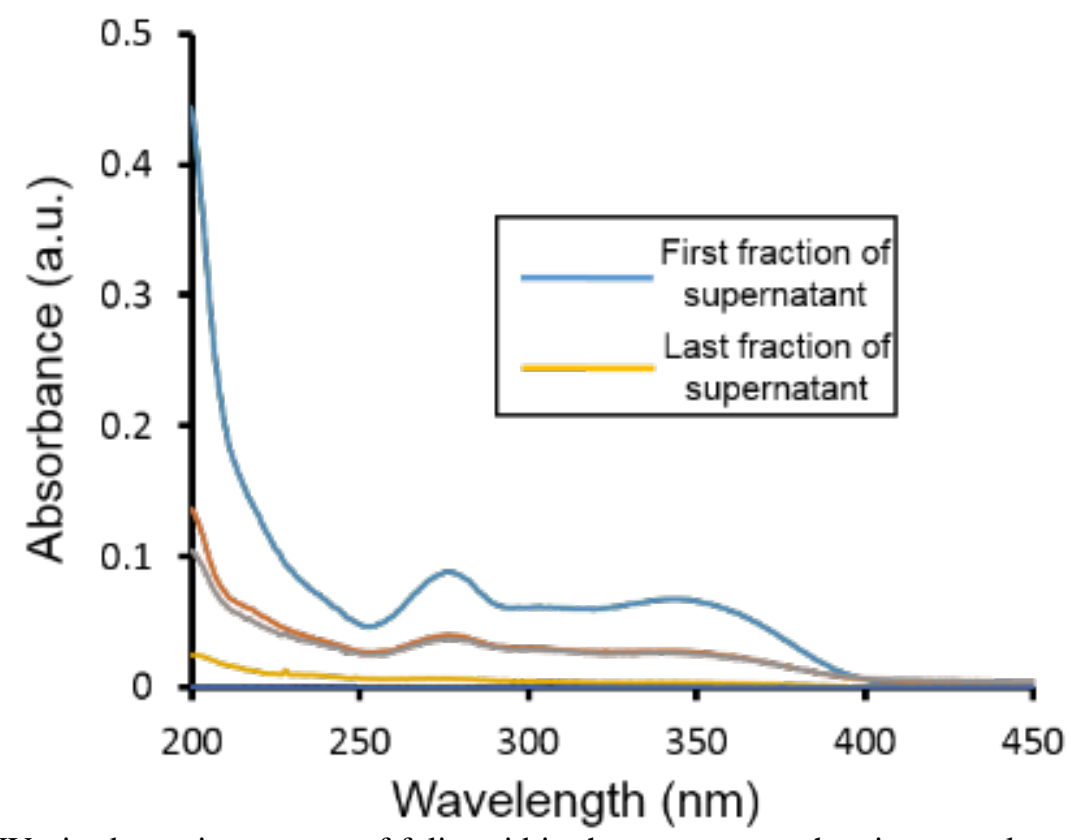

Figure S5.2. UV-vis absorption spectra of folic acid in the supernatant showing complete removal of folic acid from CPN-PEG 2000 -FA solution via dialysis (dialysis membrane, MWCO: 5000).

FTIR was utilized as the preliminary characterization method to determine whether the azide displacement and click reaction occurred. The organic azide provides a unique absorbance in the IR spectrum at $~ 2100$ $2108 \mathrm{~cm}^{-1}$. The absorption at $2107 \mathrm{~cm}^{-1}$ for $\mathbf{C P N}-\mathbf{P E G}_{\mathbf{2 0 0 0}}$-FA characteristic of the azide termini which almost disappeared in favor of a new absorptions at $1642 \mathrm{~cm}^{-1}$ and $1603 \mathrm{~cm}^{-1}$ characteristic of the amide group and triazole unsaturations, respectively. Additionally, the acid functional group of $\mathrm{PEG}_{2000}-\mathrm{FA}$ gives rise to a characteristic broad and strong stretch from 3600 to $2500 \mathrm{~cm}^{-1}$. 


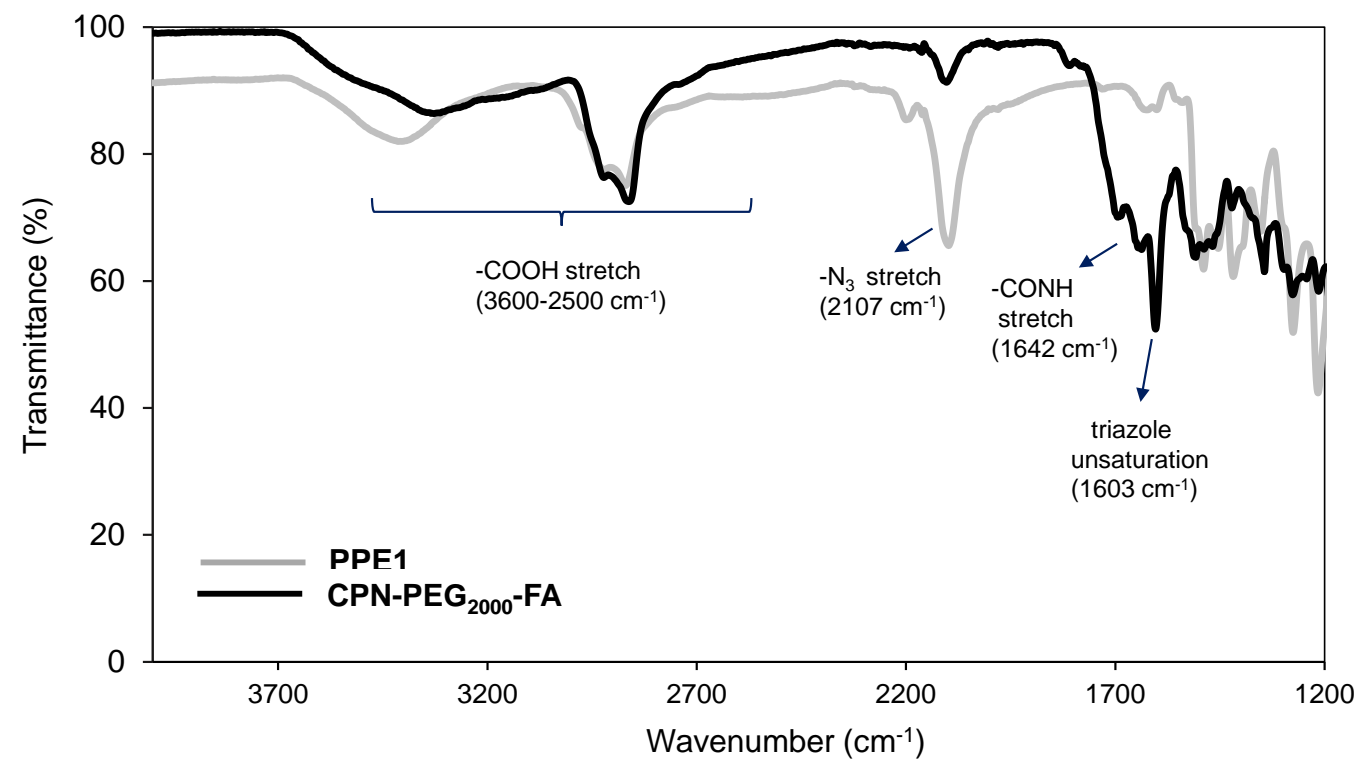

Figure S5.3. FTIR spectra of PPE1 (gray line) and CPN-PEG 2000-FA (black line).

\subsubsection{Synthesis of PPE2.}

Using the general polymerization procedure described above, polymerization of 1,4-bis(2-(2-(2bromoethoxy)ethoxy)ethoxy)-2,5-diethynylbenzene $\quad$ (M1, $26.4 \quad \mathrm{mg}, \quad 0.048 \quad \mathrm{mmol}), \quad$ 1,4-bis(2-(2azidoethoxy)ethoxy)-2,5-diiodobenzene $\quad(\mathbf{M} 2, \quad 14.15 \quad \mathrm{mg}, \quad 0.024 \quad \mathrm{mmol}), \quad$ 4,7dibromobenzo[c][1,2,5]thiadiazole $(7.07 \mathrm{mg}, 0.024 \mathrm{mmol})$ in presence of $\mathrm{Pd}\left(\mathrm{PPh}_{3}\right)_{2} \mathrm{Cl}_{2}(1.69 \mathrm{mg}, 0.0024$ mmol) and CuI (0.228 mg, $0.0012 \mathrm{mmol}$ ) yielded polymer PPE2 in 57\% (47mg). ${ }^{1} \mathrm{H}$ NMR (400 MHz, DMSO-d ${ }_{6}$ ) $\delta 8.11-7.82$ (br m, 0.45H), 7.31 (br, 1H), 7.18 (br, 2H), 4.23 (br, 6H), 3.87-3.58 (br, 27.6H), 3.27 (br, 6.6H), 1.12 (br, 6.79H). IR (neat) v 3403, 2924, 2869, 2102, 1489, 1454, 1414, 1276, 1216, $1221 \mathrm{~cm}^{-1}$. UV-Vis (THF) $\lambda_{\max }=448 \mathrm{~nm}, \varepsilon=57876.53 \mathrm{M}^{-1} \mathrm{~cm}^{-1}$ (per repeating unit), emission (THF) $\lambda_{\max }=575 \mathrm{~nm}$, Q.Y. $=0.35$ (in THF).

\subsubsection{Synthesis of CPN-PEG 2000 .}

Using the general click reaction procedure described above, reaction of PPE2(13.18 $\mathrm{mg}, 0.016 \mathrm{mmol})$ and $\alpha$-(hex-5-ynamide)- $\omega$-hydroxyl PEG 2000 (10, $34 \mathrm{mg}, 0.016 \mathrm{mmol})$, in presence of $\mathrm{CuBr}$ (0.6864 mg, 0.0048 mmol) and TEMED (0.557 mg, $0.0048 \mathrm{mmol}$ ) yielded CPN-PEG 2000. ${ }^{1} \mathrm{H}$ NMR (400 MHz, DMSO-d 6 ) $\delta$ 7.87 (t, 1H), 7.29-7.17 (br, 4H), 4.56 (t, 0.5H), 4.23 (br, 6H), 3.85-3.67 (br, 26H), 3.50 (br, 212H), 2.15 (m, 
4H), 1.65 (m, 2H), 1.13 (br, 9H). IR (neat) v 3600-2500 (br), 2104, 1642, 1603, 1492, 1454, 1342, 1275, $1101 \mathrm{~cm}^{-1}$. UV-Vis $\left(\mathrm{H}_{2} \mathrm{O}\right) \lambda_{\max }=480 \mathrm{~nm}$, emission $\left(\mathrm{H}_{2} \mathrm{O}\right) \lambda_{\max }=620 \mathrm{~nm}, \mathrm{Q} . \mathrm{Y} .=0.48$ (in water).

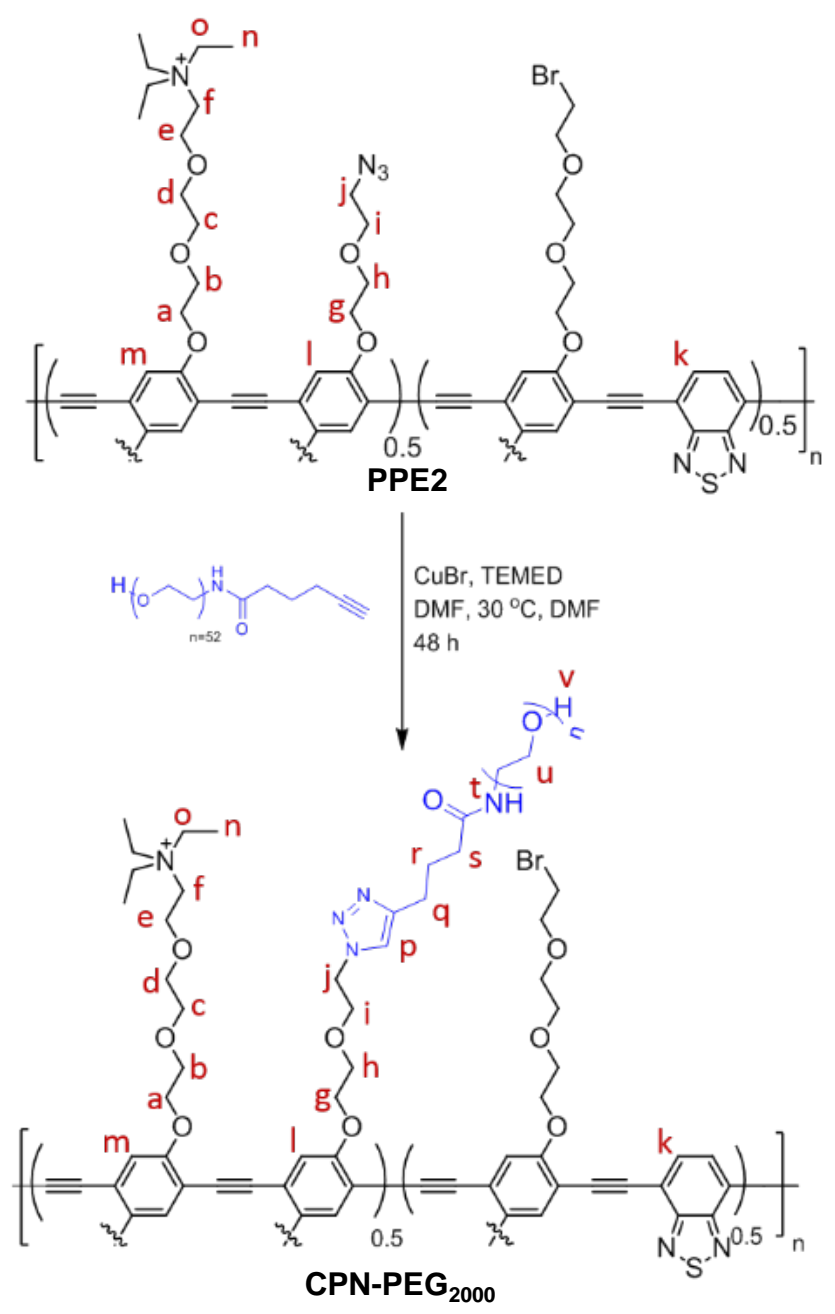

Scheme S5.3. Click reaction of alkyne end-functionalized PEG 2000 onto azide end-functionalized PPE2 to yield CPN-PEG 2000 .

\subsubsection{Characterization of PPE2 and CPN-PEG 2000 .}

A similar "click reaction" strategy was employed to synthesize CPN-PEG 2000 (Scheme S5.3). After purification via dialysis and freeze drying, a red solid powder was collected which was used for nanoparticle preparation. ${ }^{1} \mathrm{H}$ NMR integral ratio between PPE2 and alkynyl ended $\mathrm{PEG}_{2000}(\mathbf{1 0})$ indicates incorporation complete of PEG $_{2000}$ into the polymer chain. 


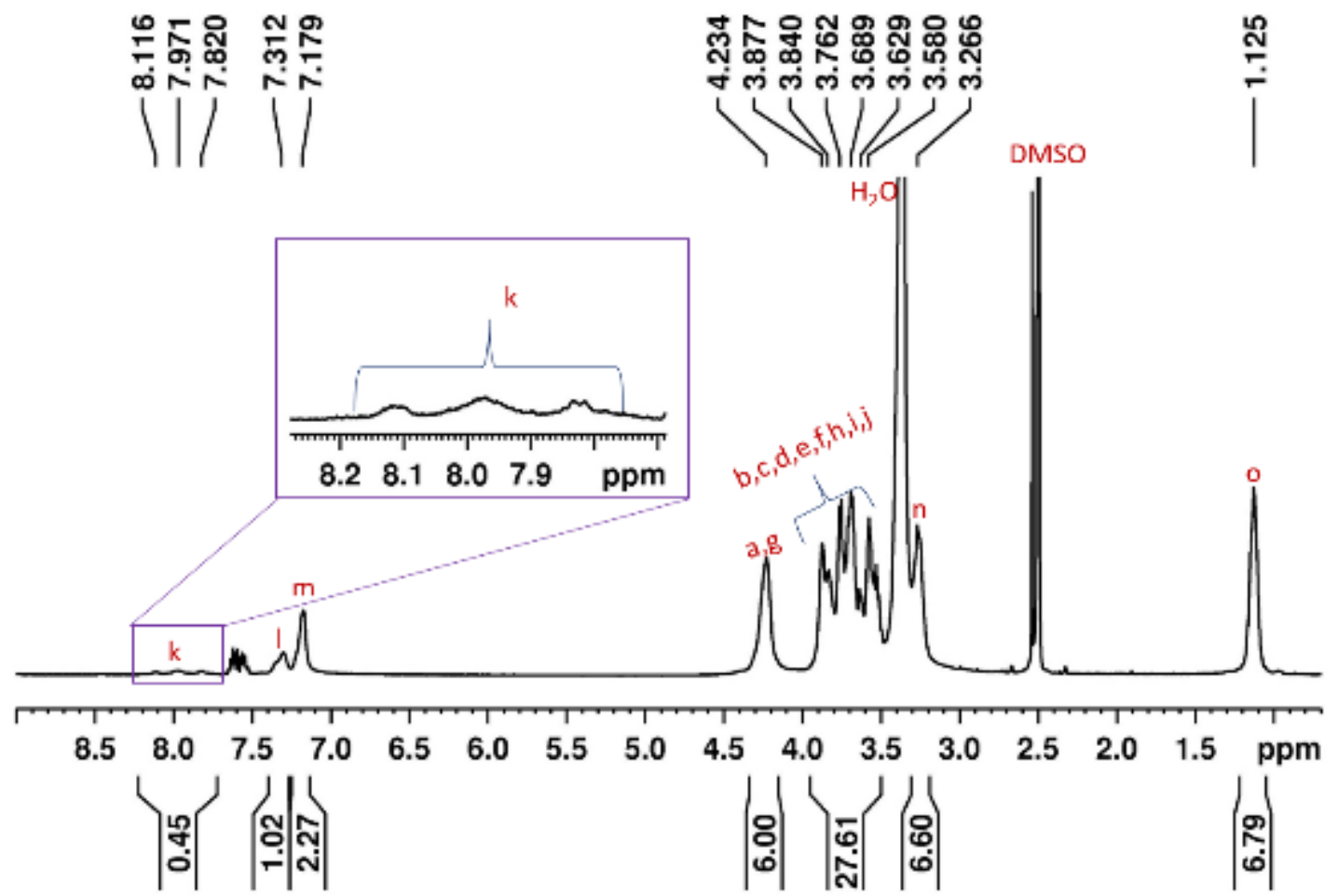

Figure S5.4. ${ }^{1} \mathrm{H}$ NMR spectrum of PPE2 in DMSO- $\mathrm{d}_{6}$.

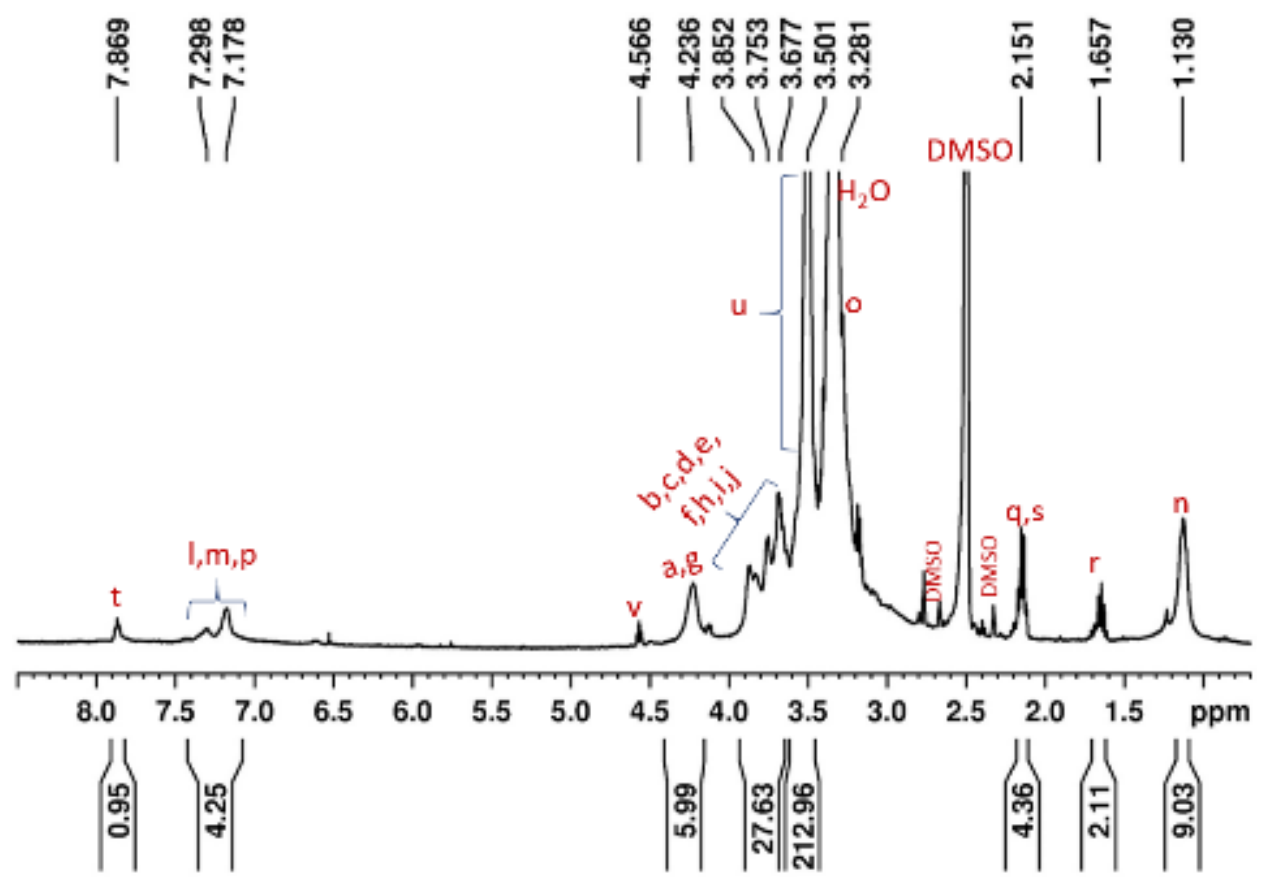

Figure S5.5. ${ }^{1} \mathrm{H}$ NMR spectra of $\mathbf{C P N}-\mathbf{P E G}_{2000}$ in DMSO-d $\mathrm{d}_{6}$. 


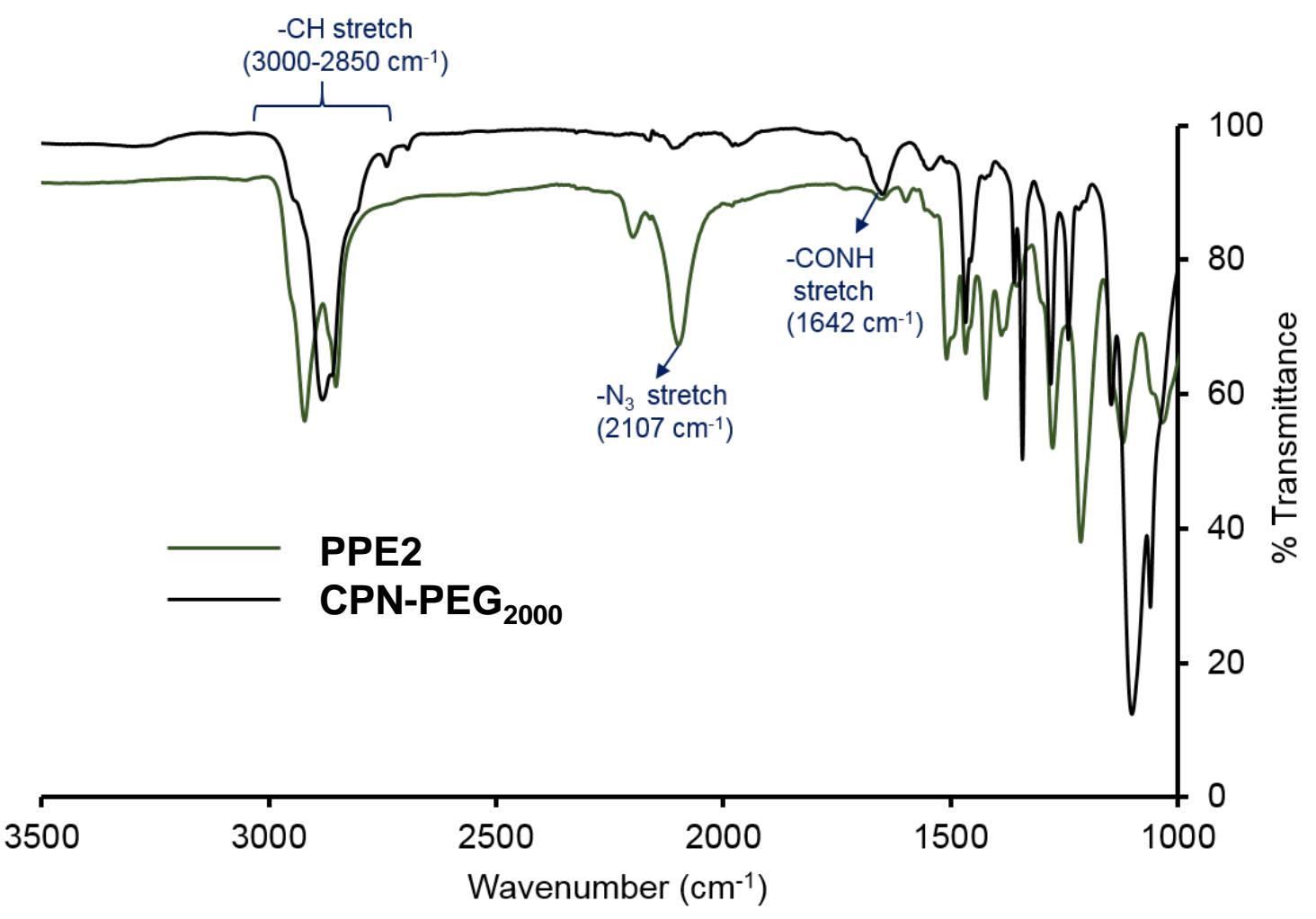

Figure S5.6. FTIR spectra of PPE2 (green line) and CPN-PEG 2000 (black line).

\subsubsection{Nanoparticle preparation.}

PEG 2000 -folic acid grafted conjugated polymers were dissolved in HPLC grade DMSO by stirring overnight.

The conjugated polymer nanoparticles were prepared by adding the stock solution in deionized water with vigorous stirring. DMSO and unreacted $\mathrm{PEG}_{2000}$-Folic acid were removed by washing the water dispersible with HPLC grade dichloromethane. Finally, the nanoparticles were filtered through a $0.5 \mu \mathrm{m}$ PTFE syringe filters.

\subsubsection{Determination of hydrodynamic diameter of CPNs.}

Light scattering measurements were performed using a NanoSight LM10 HS Instrument (Nanosight Ltd., Amesbury, UK) equipped with a sCMOS camera using deionized water ( $\mathrm{DI}_{2} \mathrm{O}, 18 \Omega$ ) as dispersant. A Class 3B laser (488 nm blue laser) source was used as the light source and all experiments were performed at a temperature of $25^{\circ} \mathrm{C}$ monitored by a LM14C temperature controller (NanoSight, Amesbury, United Kingdom). CPN samples in water were filtered through PTFE syringe filters (cut-off $0.45 \mu \mathrm{m}$ ) prior to injecting in the sample chamber to remove dust or any larger aggregates. Samples were then injected into the 
chamber with $1 \mathrm{~mL}$ sterile syringes (Restek Corporation, Pennsylvania, USA) until the liquid reached the tip of the nozzle. A video of typically 60 seconds duration was taken, with a frame rate of 30 frames per second. Each video was then analyzed by NTA software (NanoSight Ltd.) to give the mean, mode, and standard deviation of nanoparticle size distribution together with an estimate of concentration (particles $/ \mathrm{mL}$ ). Each sample was measured three times.

\subsubsection{Determination of zeta potential of CPNs.}

Zeta potential of CPNs were measured by Zetasizer nano-ZS (Zen 3600, Malvern Instruments Ltd.) using a folded disposable capillary cell (Catalog \# DTS1060) at $25^{\circ} \mathrm{C}$. The nanoparticle dispersions (in DI water) were concentrated to approximately $0.5 \mathrm{mM}$ to achieve reliable results. Instrument settings were automatically determined by Malvern technology software on a sample-to-sample basis for measurement position (in mm), attenuator intensity (laser power), and number of runs per measurement. Each sample was measured six times.

\subsubsection{General cell culture.}

HeLa cells (human cervical cancer, purchased from ATCC) were seeded into a 100 x 20 mm style sterile tissue culture dish (\#353003 BD Falcon, Durham, NC, USA). The cells were grown in minimum essential medium (MEM)/Earle's balanced salt solution (EBSS) (400 $\mu$ L, HyClone, SH30024) medium supplemented with $10 \%$ fetal bovine serum (FBS), and $100 \mu \mathrm{g} / \mathrm{mL}$ penicillin and $100 \mu \mathrm{g} / \mathrm{mL}$ streptomycin for $24 \mathrm{~h}$ under $5 \% \mathrm{CO}_{2} / 95 \%$ air at $37{ }^{\circ} \mathrm{C}$. The cells were continuously maintained in the culture medium and subcultured every $48 \mathrm{~h}$.

\subsubsection{Cellular toxicity study.}

HeLa cells (ca. 10,000 cells per well), in $200 \mu \mathrm{L}$ of complete medium, were seeded into a 96-well plate and allowed to attach for one day at $37^{\circ} \mathrm{C}$ under a humidified atmosphere of $5 \% \mathrm{CO}_{2} / 95 \%$ air. Stock solution of CPNs were added into complete media and diluted to the required amount of CPNs. After addition of CPNs, the cells were incubated for another $18 \mathrm{~h}$. Cells were washed with warm PBS and incubated with fresh medium containing MTT (5 mg mL ${ }^{-1}$ in PBS, CALBIOCHEM, Germany) for $4 \mathrm{~h}$ at $37^{\circ} \mathrm{C}$. Dimethyl sulfoxide (100 $\mu \mathrm{L}$ ) was added to solubilize the purple formazan crystals formed by proliferating cells. Absorbance was measured by a microplate well reader (infinite M1000 PRO, TECAN, Switzerland) at $590 \mathrm{~nm}$. Relative cell 
viability (\%) as a function of CPNs concentration was expressed as a percentage relative to the untreated control cells. All measurements were performed in triplicate and standard deviation was included in the error bar.

\subsubsection{Cell uptake studies by flow cytometry.}

HeLa cells were seeded into a 12-well plate ( 40,000/well) one day prior to CPN treatment. Cells were then incubated with $6 \mu \mathrm{M}$ of CPN for 1, 2, 4, 8, and 18h. For complexation with HA, CPN was mixed with HA (40K and $100 \mathrm{~K}$ ) at molar ratios (based on repeating unit of CPN and HA) of 1:0 (control), 1:1, 1:2, and 1:5. The CPN/HA complexes were allowed to incubate approximately 30 minutes prior to incubating with cells. After incubation with cells, samples were treated with trypan blue $(200 \mu \mathrm{M})$ for 10 minutes at $37^{\circ} \mathrm{C}$ and washed three times with 1X PBS. Cells were detached with trypsin/EDTA and fixed with $4 \%$ paraformaldehyde for 10 minutes at room temperature, then resuspended in flow cytometry buffer (1X PBS containing $5 \%$ bovine serum albumin and $0.02 \%$ sodium azide). 10,000 events per measurement were recorded within the gate of control cells, which was selected based on forward and side scattering of control cells to eliminate data collection from dead cells and artifacts. Mean fluorescence intensity of CPNs (FL3 channel, 590-620 nm wavelength range) was obtained using three independent sample sets and accounting for variation in cell autofluorescence. For uptake plots, values were normalized to control cells.

\subsubsection{Cell inhibition assays.}

For endocytosis pathway studies, HeLa cells were seeded into a 12-well plate ( $40,000 /$ well) one day prior to CPN treatment. Cells were then treated without (control) or in the presence of pharmaceutical inhibitors chlorpromazine $(24 \mu \mathrm{M})$ for clathrin mediated endocytosis (CME), genistein $(210 \mu \mathrm{M})$ for caveolae mediated endocytosis (CvME), methyl- $\beta$-cyclodextrin $(1000 \mu \mathrm{M})$ for CME and macropinocytosis (CvME/MPC), LY294002 $(120 \mu \mathrm{M})$ for macropinocytosis (MPC1), or cytochalasin D $(40 \mu \mathrm{M})$ for macropinocytosis (MPC2) for 30 minutes before CPN treatment. For blocking any potential pathways of FA and HA, cells were treated with excess FA $(1 \mathrm{mM})$ and HA $(100 \mathrm{~K}, 1.5 \mathrm{mM})$ for one hour before CPN treatment. After CPN treatment, cells were prepared as discussed in previous section. For inhibition assays, values were normalized to $\mathrm{CPN}$. 


\subsubsection{Cell uptake studies by microscopy.}

HeLa cells were seeded into a 12-well plate ( 20,000/well) with glass coverslip (\#1254584, Fisher Scientific) one day prior to CPN treatment and cultured in a minimum essential medium (MEM)/Earle's balanced salt solution (EBSS) $(500 \mu \mathrm{L})$ medium containing 10\% fetal bovine serum (FBS) and $100 \mathrm{U} / \mathrm{m}$ penicillin for 24 h under $5 \% \mathrm{CO}_{2}$ at $37{ }^{\circ} \mathrm{C}$. CPNs and $\mathrm{CPN} / \mathrm{HA}$ complexes were prepared in fresh culture medium $(500 \mu \mathrm{L})$, and added to cells after washing with $1 \mathrm{X}$ PBS and removing media, then cultured for 4, 8, and $18 \mathrm{~h}$ (final CPN concentration: $6 \mu \mathrm{M}$ ). After incubation, cell were washed with PBS and fixed with 4\% paraformaldehyde for $10 \mathrm{~min}$. After washing with $1 \mathrm{X}$ PBS, a $1 \mu \mathrm{L}$ aliquot of Hoechst $(5 \mu \mathrm{g} / \mathrm{mL})$ was added to $1 \mathrm{X}$ PBS and incubated with the cells for $10 \mathrm{~min}$ at room temperature. Cells were then washed three times with 1X PBS and coverslips were mounted on microscope slides (\#1125441, Fisher Scientific) using a 1:1 glycerol/PBS mounting medium. Fluorescent images of the cells were obtained using a DeltaVision Elite Microscope System (Applied Precision, Issaquah, Washington, USA) equipped with bandpass filters such as blue (410-460 nm, Hoechst) and red (595-635nm, CPNs) using a 60X oil immersion lens (NA 1.42) and $\eta$

$=1.520$ immersion oil . Top and bottom of the chosen cells was identified, and a Z-stack plot was imaged for each channel. Deconvolution of each channel was performed using the microscope software (Softworx 5.0 application, Applied Precision, Issaquah, Washington, USA) and supplied method file using 50 iterations per channel and high noise filtering setting. 


\subsubsection{Characterization data.}

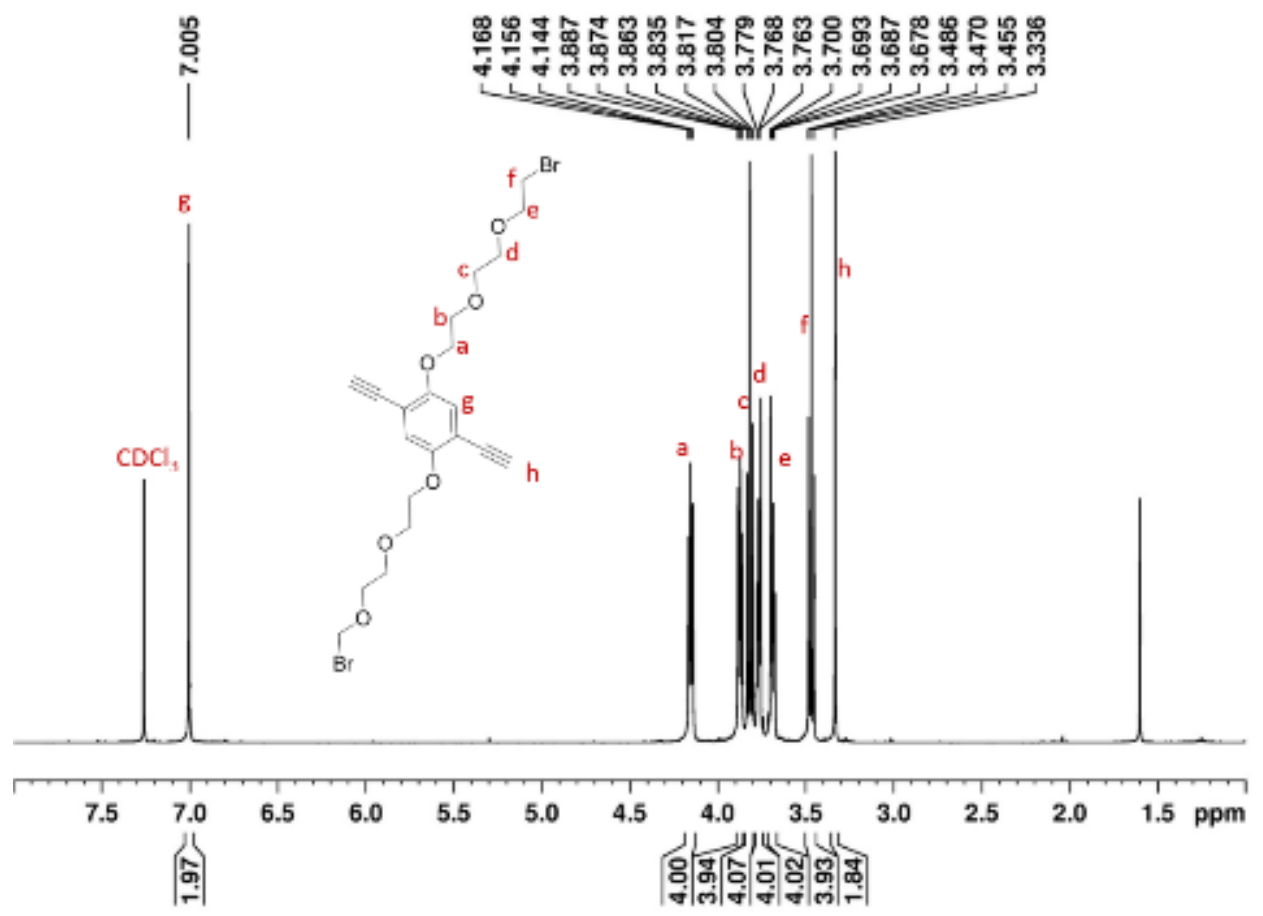

Figure S5.7. ${ }^{1} \mathrm{H}$ NMR spectrum of $\mathbf{M} 1$ in $\mathrm{CDCl}_{3}$.

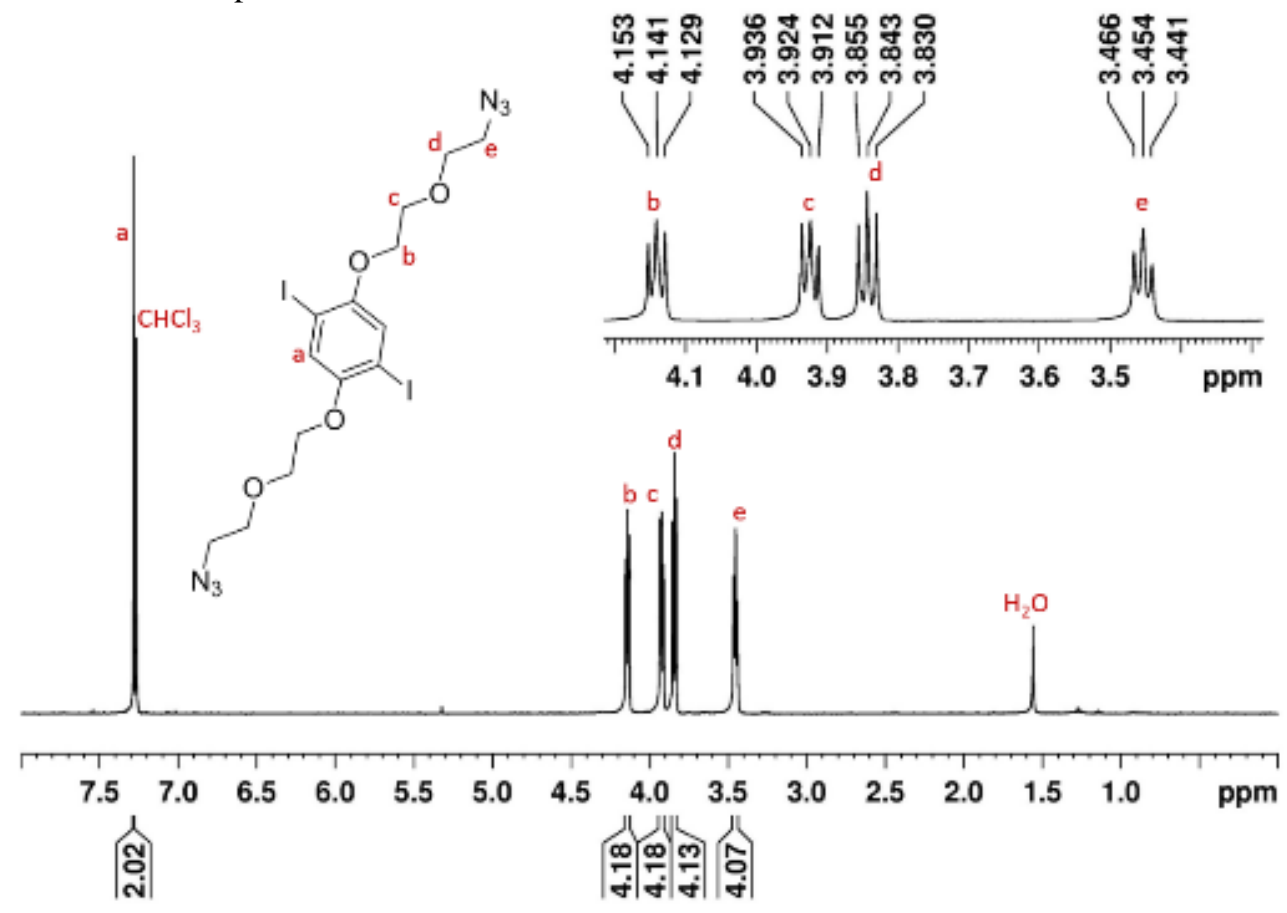

Figure S5.8. ${ }^{1} \mathrm{H}$ NMR spectrum of $\mathbf{M} 2$ in $\mathrm{CDCl}_{3}$. 


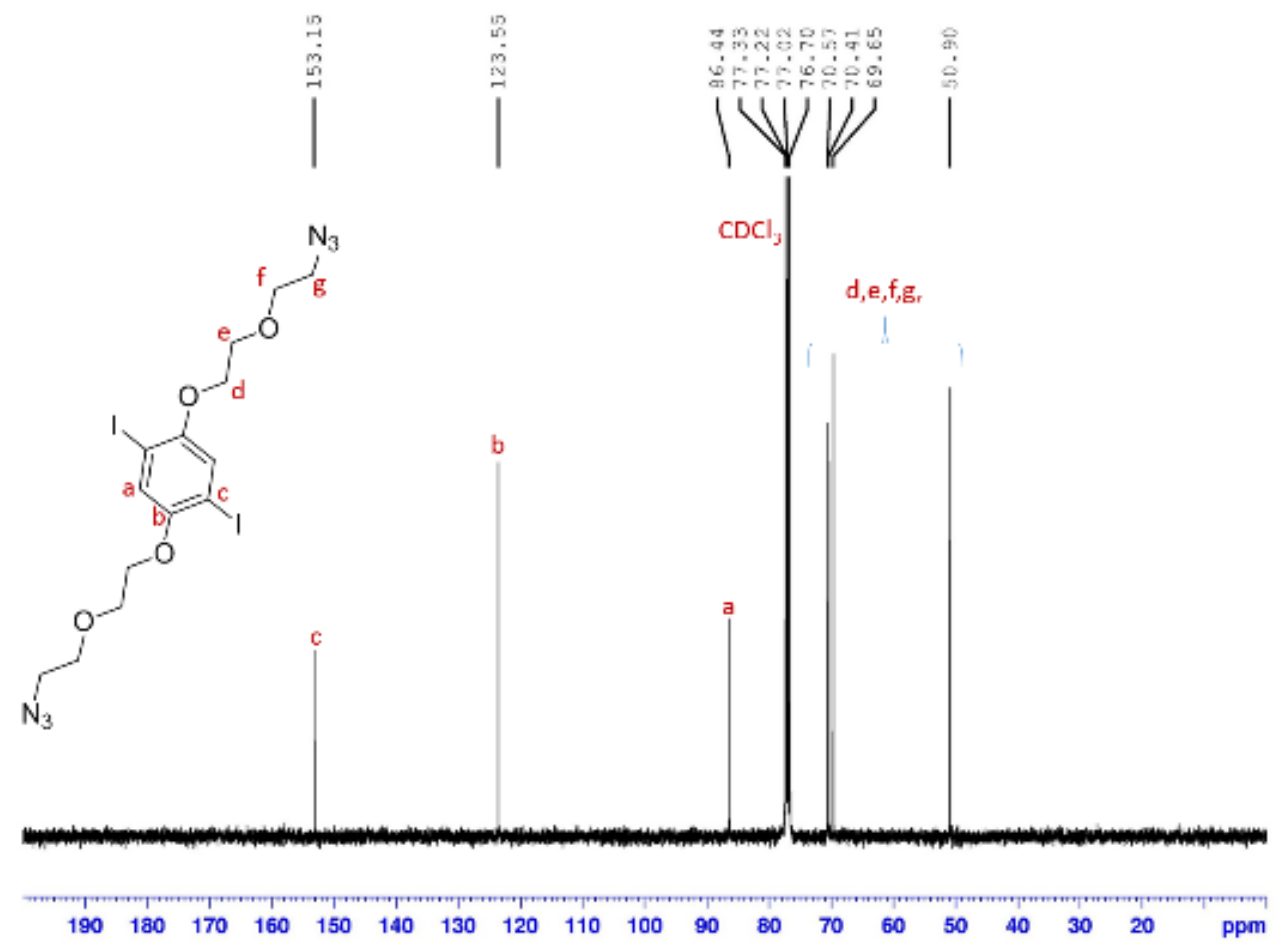

Figure S5.9. ${ }^{13} \mathrm{C}$ NMR spectrum of $\mathbf{M} 2$ in $\mathrm{CDCl}_{3}$.

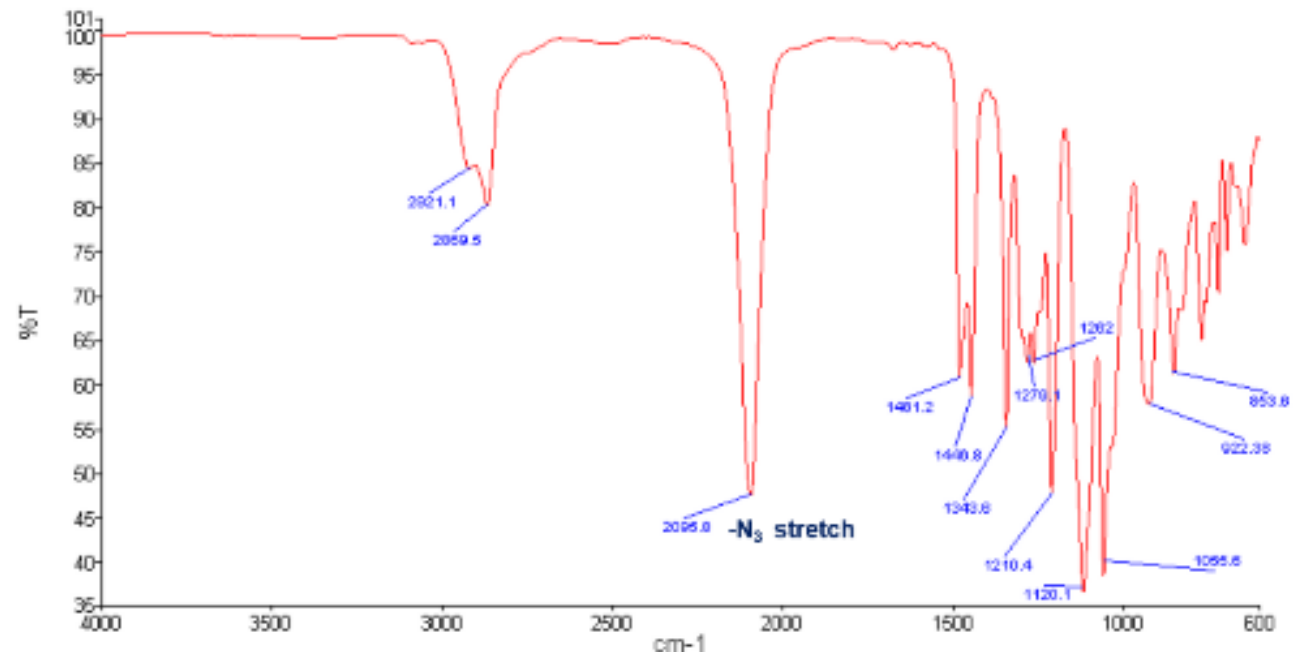

Figure S5.10. IR Spectrum of $\mathbf{M} 2$ showing $-\mathrm{N}_{3}$ stretch at $2095.8 \mathrm{~cm}^{-1}$. 


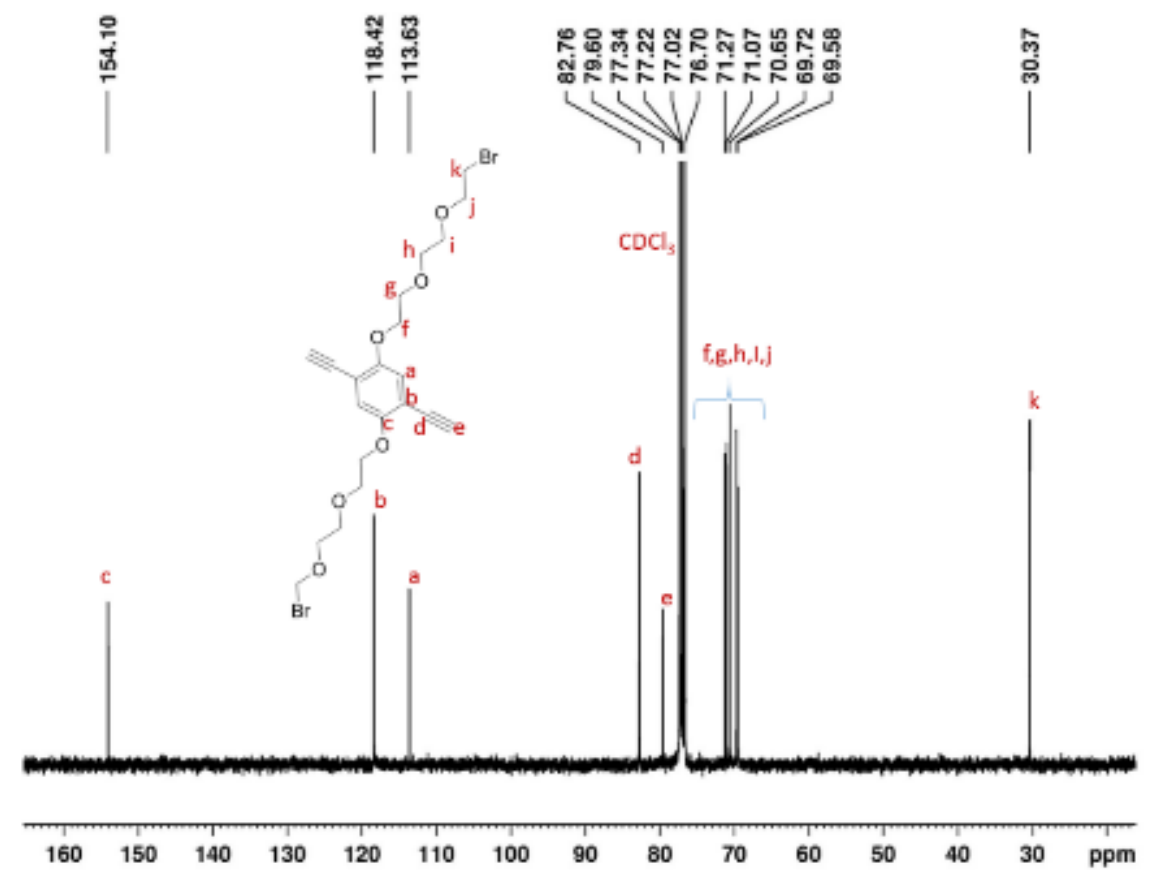

Figure S5.11. ${ }^{13} \mathrm{C}$ NMR spectrum of $\mathbf{M 1}$ in $\mathrm{CDCl}_{3}$.

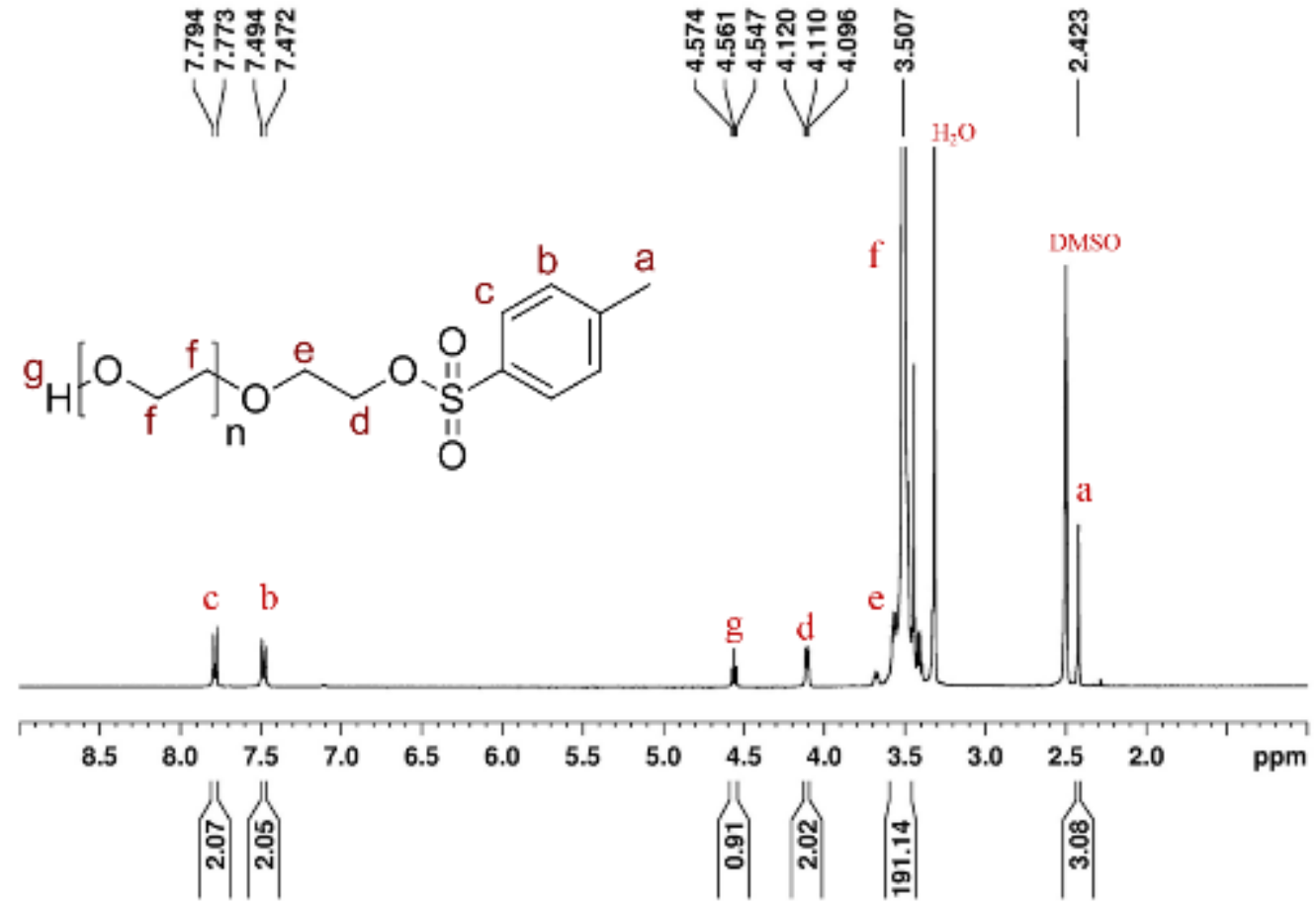

Figure S5.12. ${ }^{1} \mathrm{H}$ NMR spectrum of 7 in DMSO-d ${ }_{6}$. 


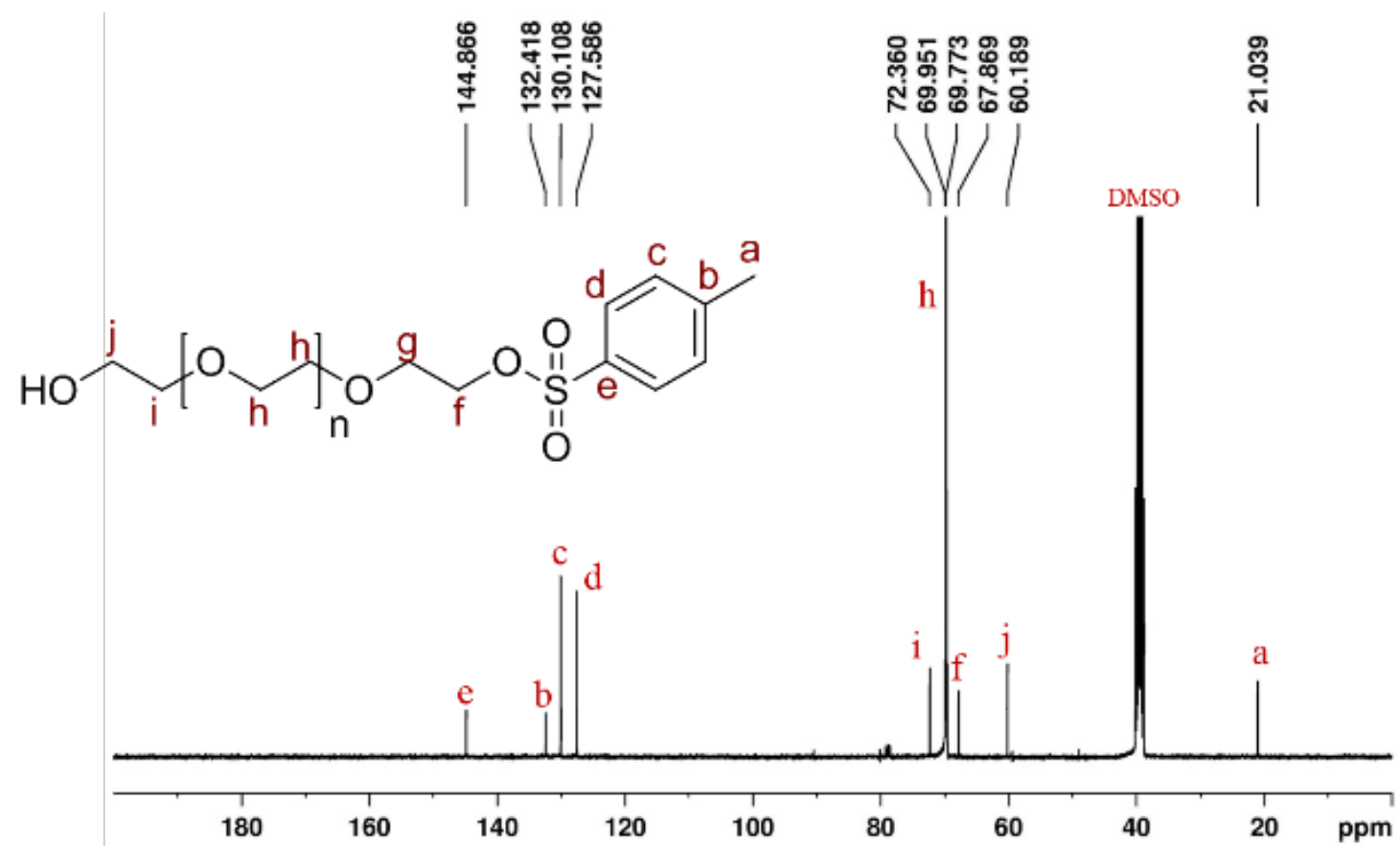

Figure S5.13. ${ }^{13} \mathrm{C}$ NMR spectrum of 7 in DMSO-d 6 .

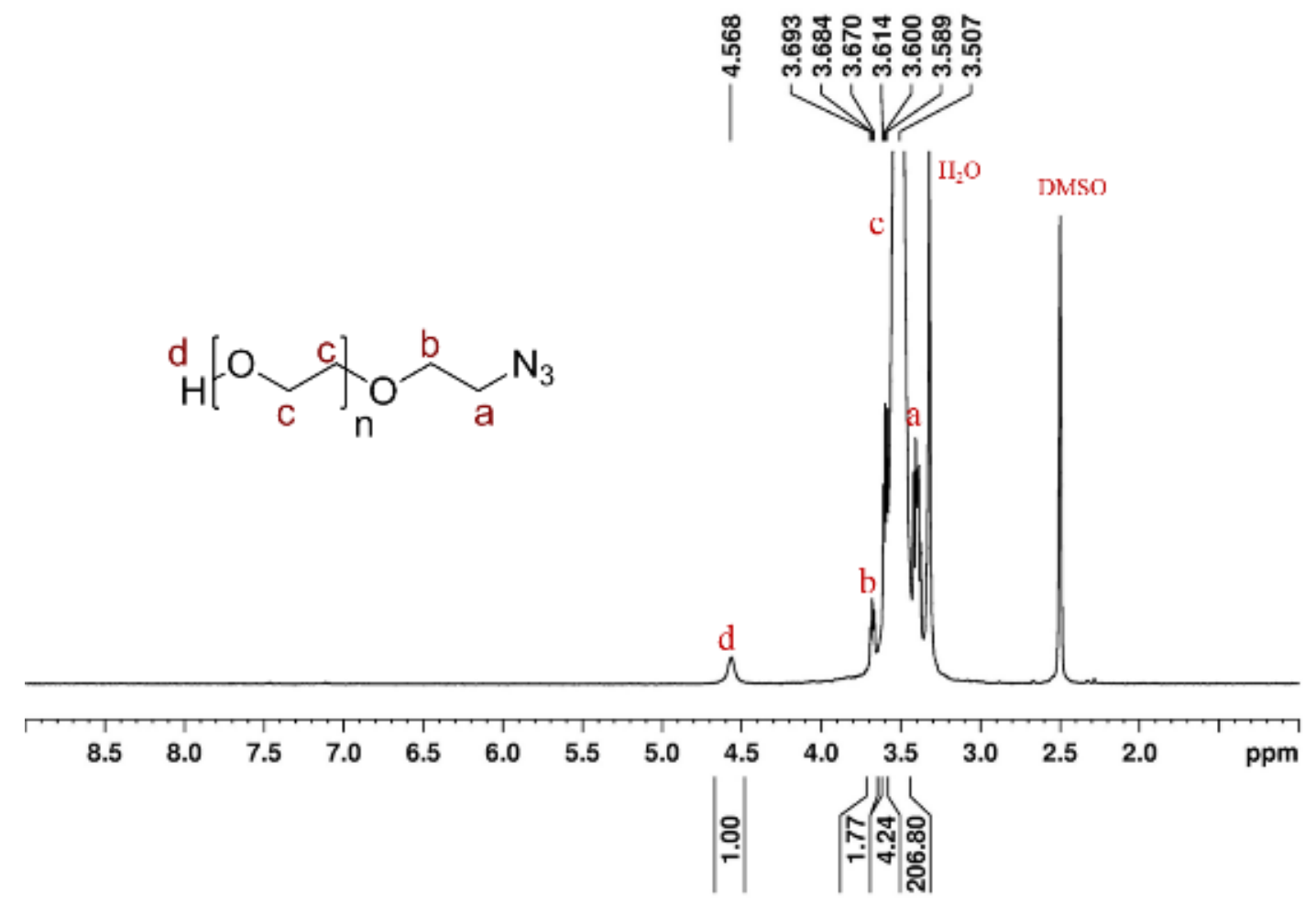

Figure S5.14. ${ }^{1} \mathrm{H}$ NMR spectrum of 8 in DMSO-d ${ }_{6}$. 


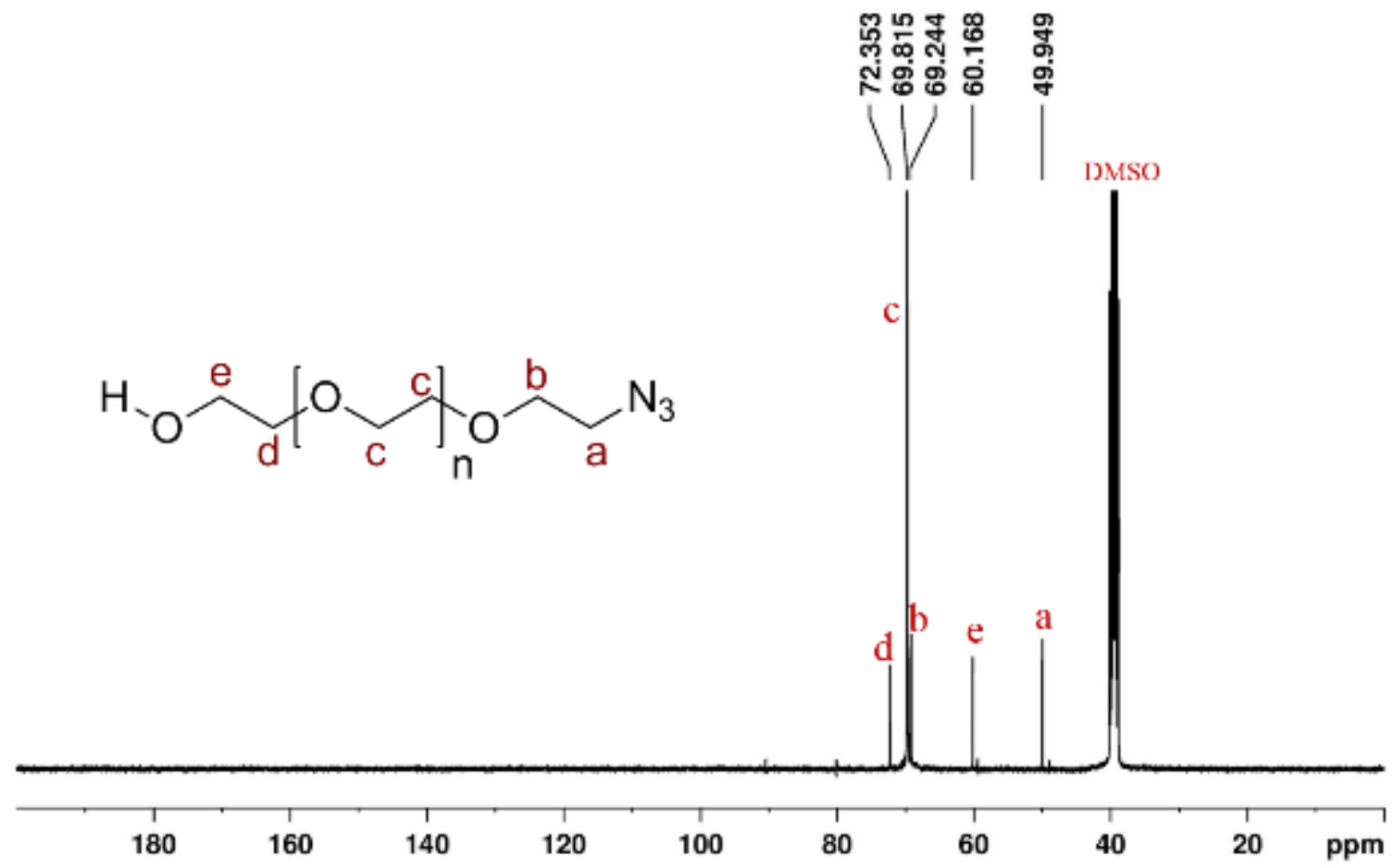

Figure S5.15. ${ }^{13} \mathrm{C}$ NMR spectrum of 8 in DMSO- $\mathrm{d}_{6}$.

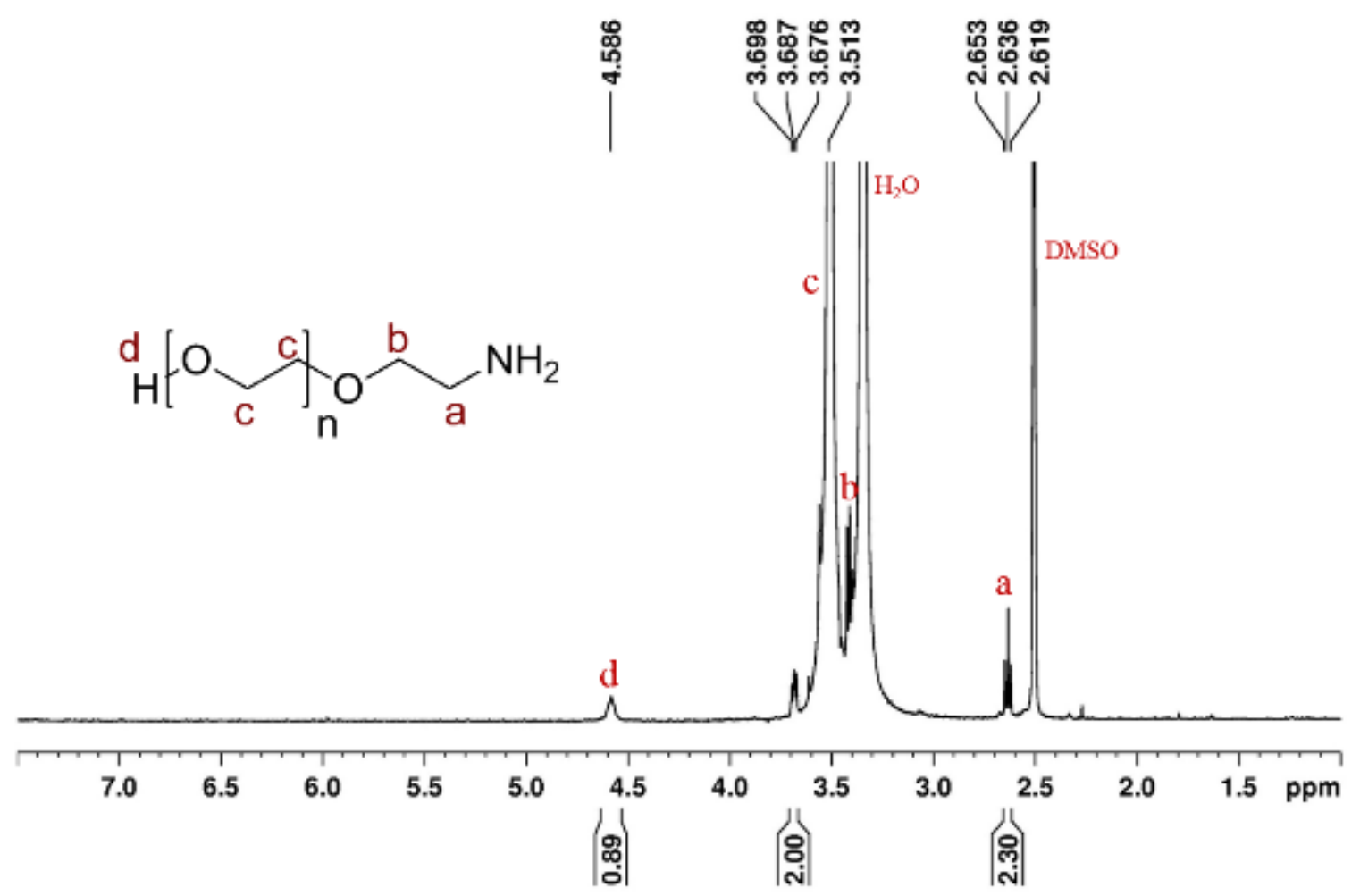

Figure S5.16. ${ }^{1} \mathrm{H}$ NMR spectrum of 9 in DMSO-d ${ }_{6}$. 


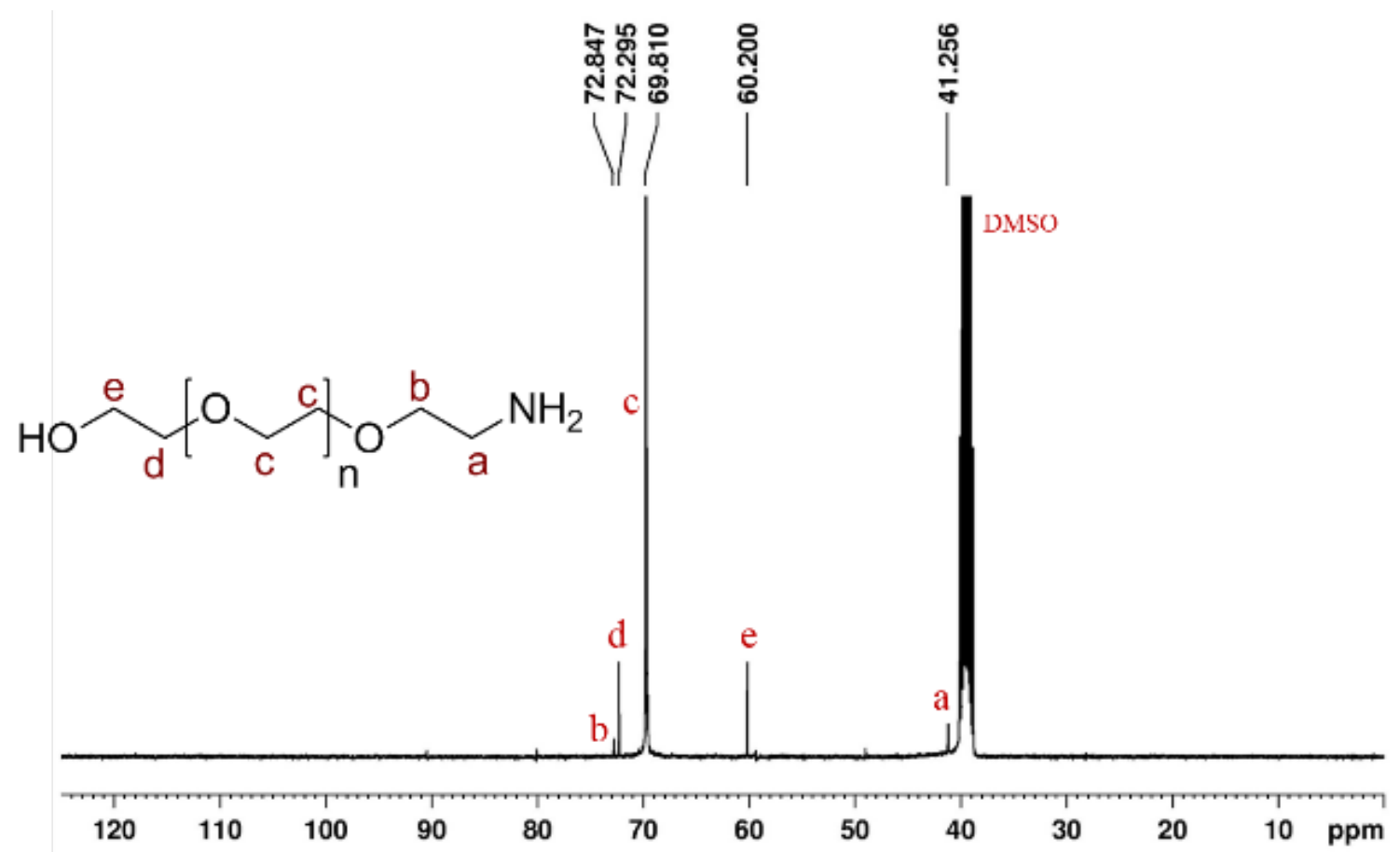

Figure S5.17. ${ }^{13} \mathrm{C}$ NMR spectrum of 9 in DMSO- $\mathrm{d}_{6}$.

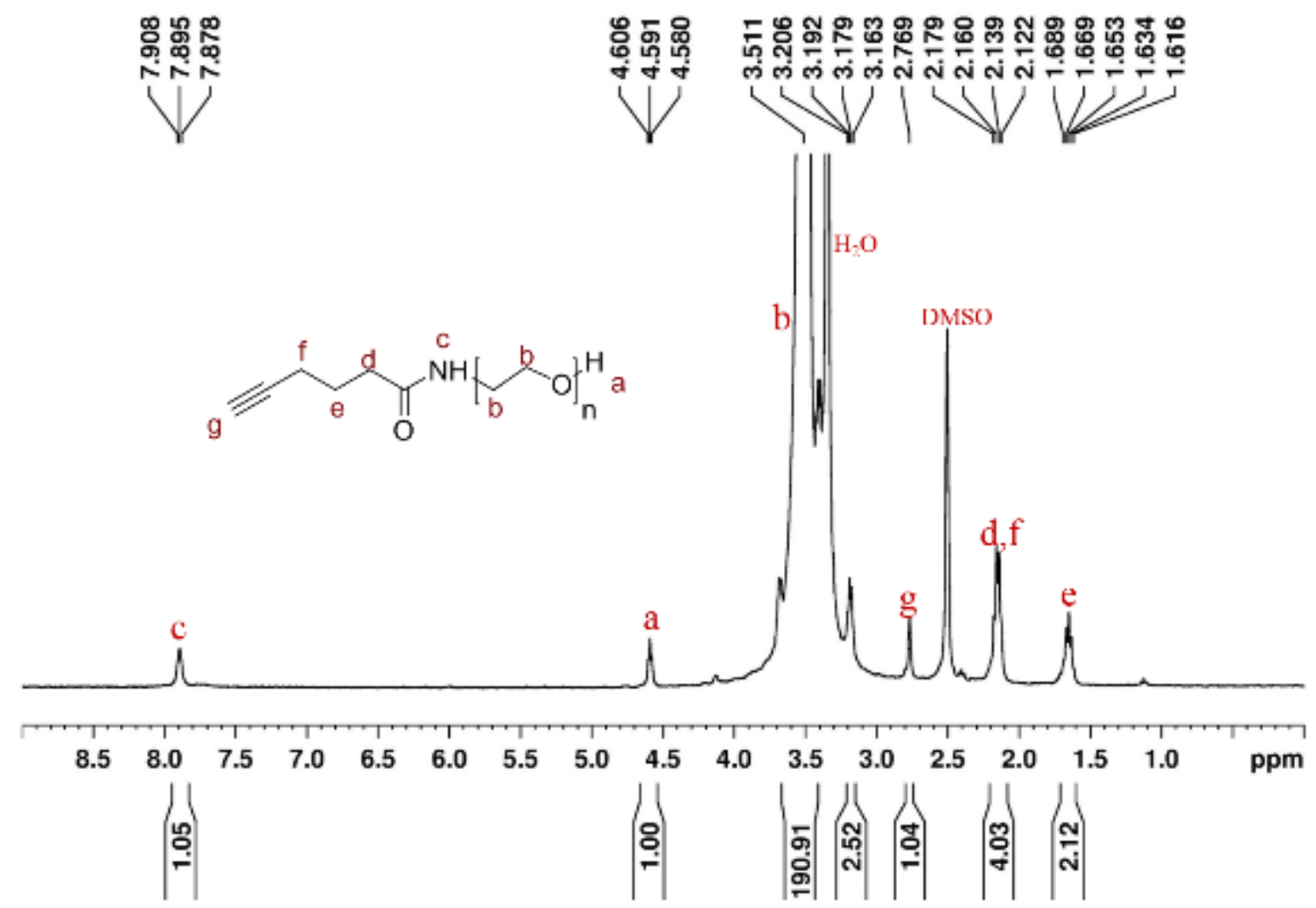

Figure S5.18. ${ }^{1} \mathrm{H}$ NMR spectrum of $\mathbf{1 0}$ in DMSO- $\mathrm{d}_{6}$. 


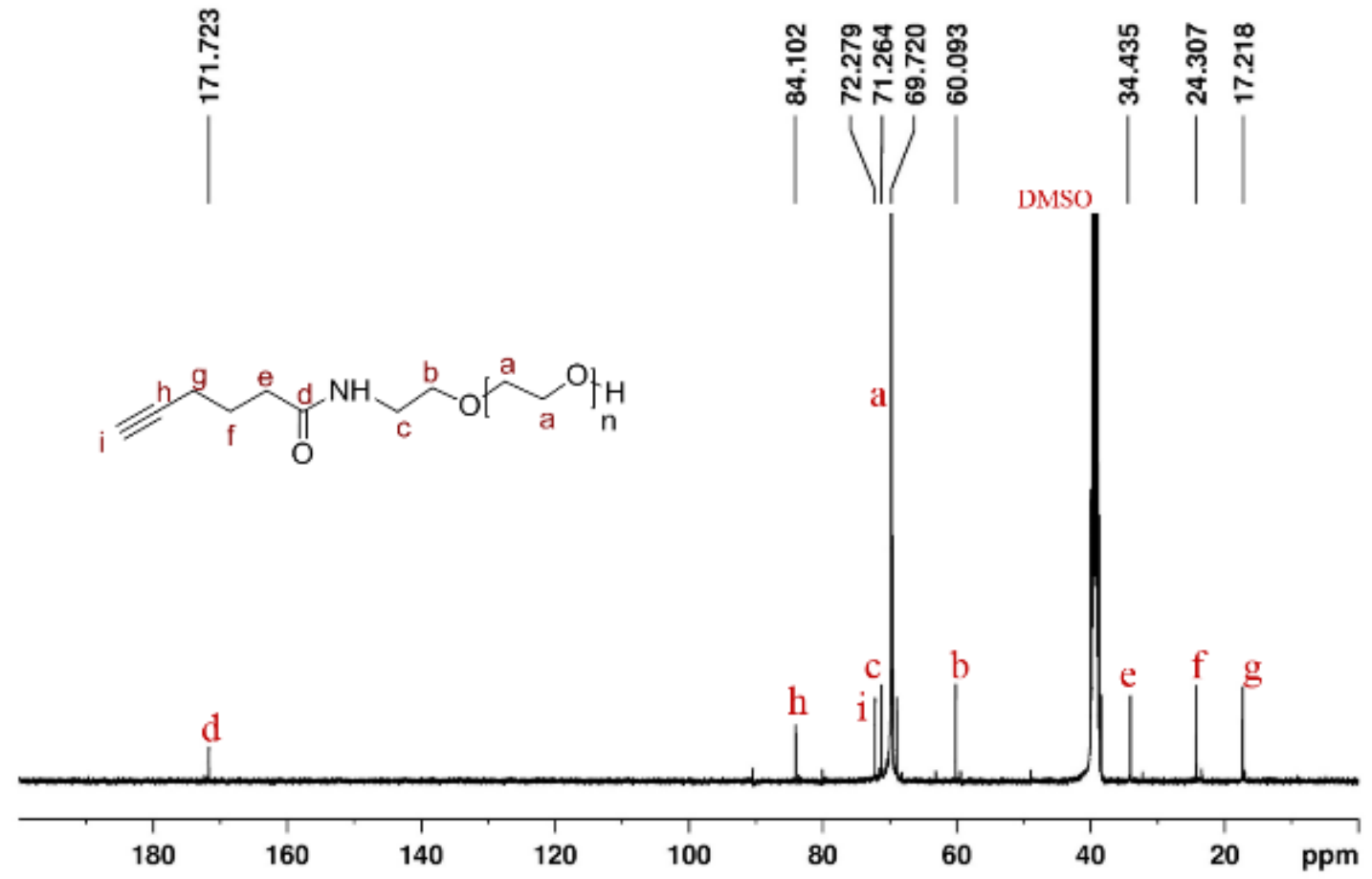

Figure S5.19. ${ }^{13} \mathrm{C}$ NMR spectrum of $\mathbf{1 0}$ in DMSO- $\mathrm{d}_{6}$.

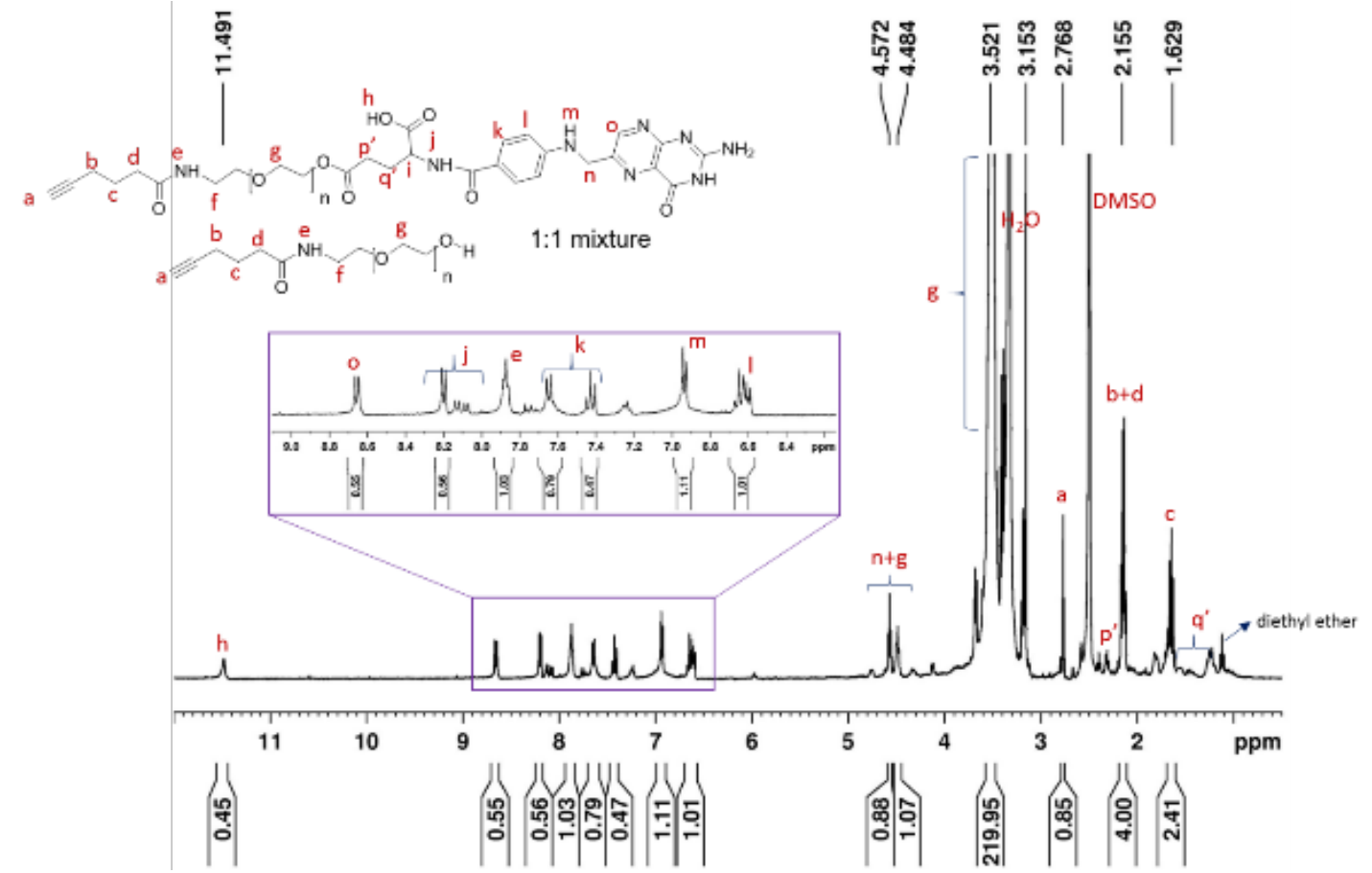

Figure S5.20. ${ }^{1} \mathrm{H}$ NMR spectrum of 1:1 mixture of $\mathbf{M} 3$ and $\mathbf{1 0}$ in DMSO-d 6 . 


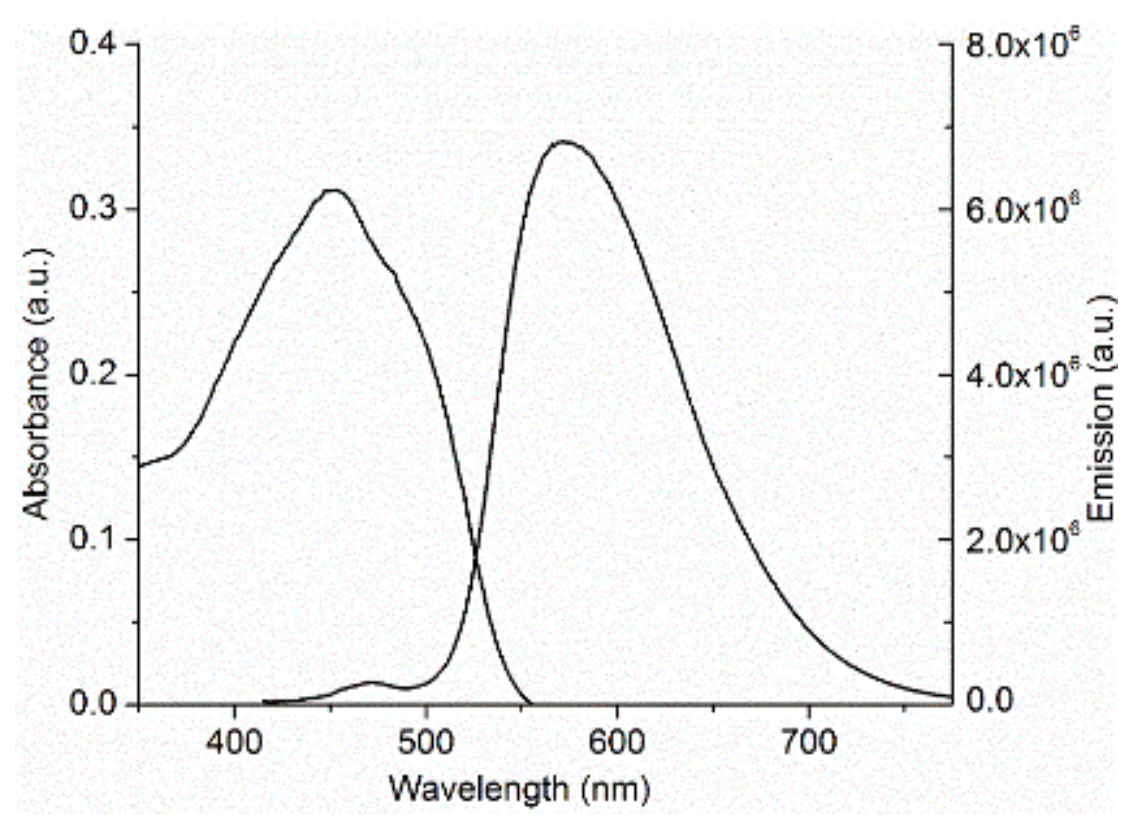

Figure S5.21. Absorption and emission spectra for PPE2 in THF (Excitation $=400 \mathrm{~nm}$, slit width $=2 \mathrm{~nm}$, integration time $=0.1 \mathrm{~s}$ ).
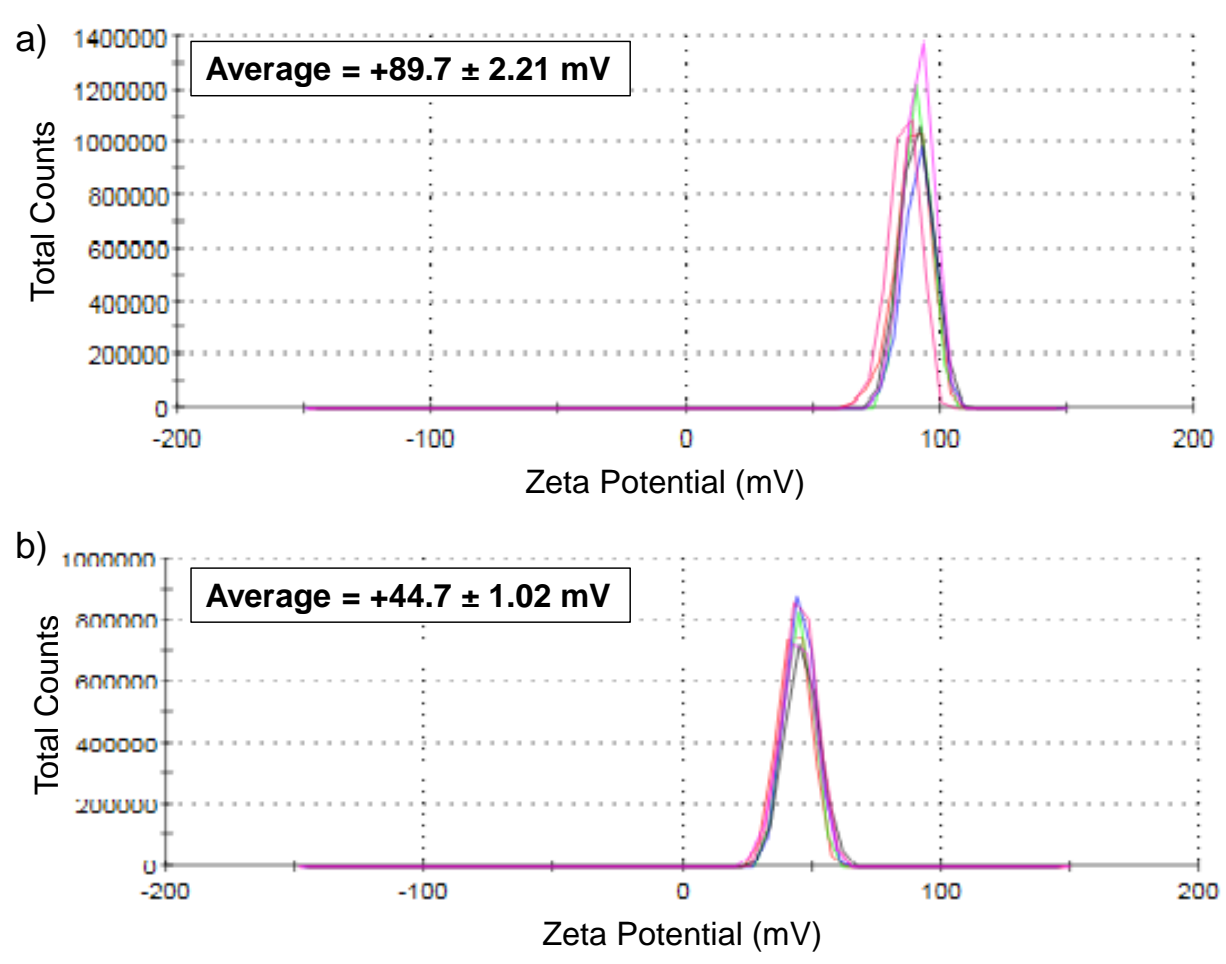

Figure S5.22. Zeta potential for CPN-PEG $2000-F A$ (a) and $\mathbf{C P N}_{\mathbf{P}} \mathbf{P E G}_{2000}$ (b). 


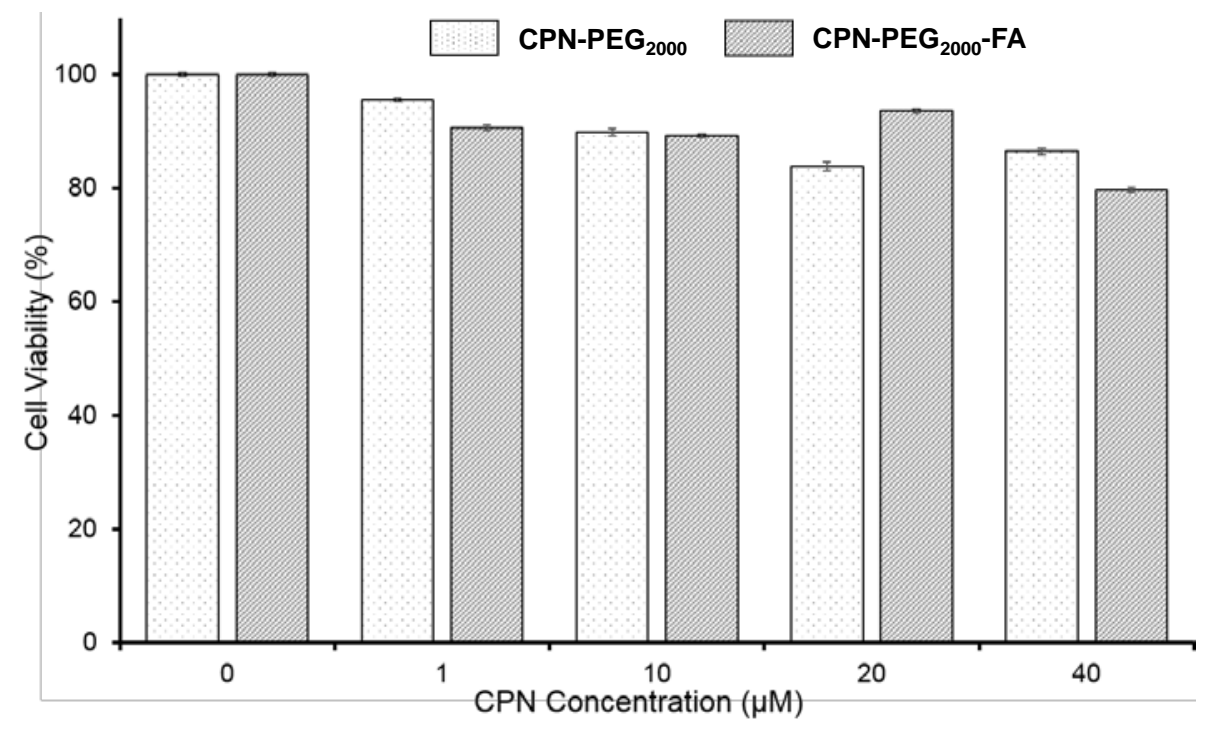

Figure S5.23. Toxicity of CPNs toward HeLa cells. Cell viability against varying concentration of CPNs is plotted as columns.

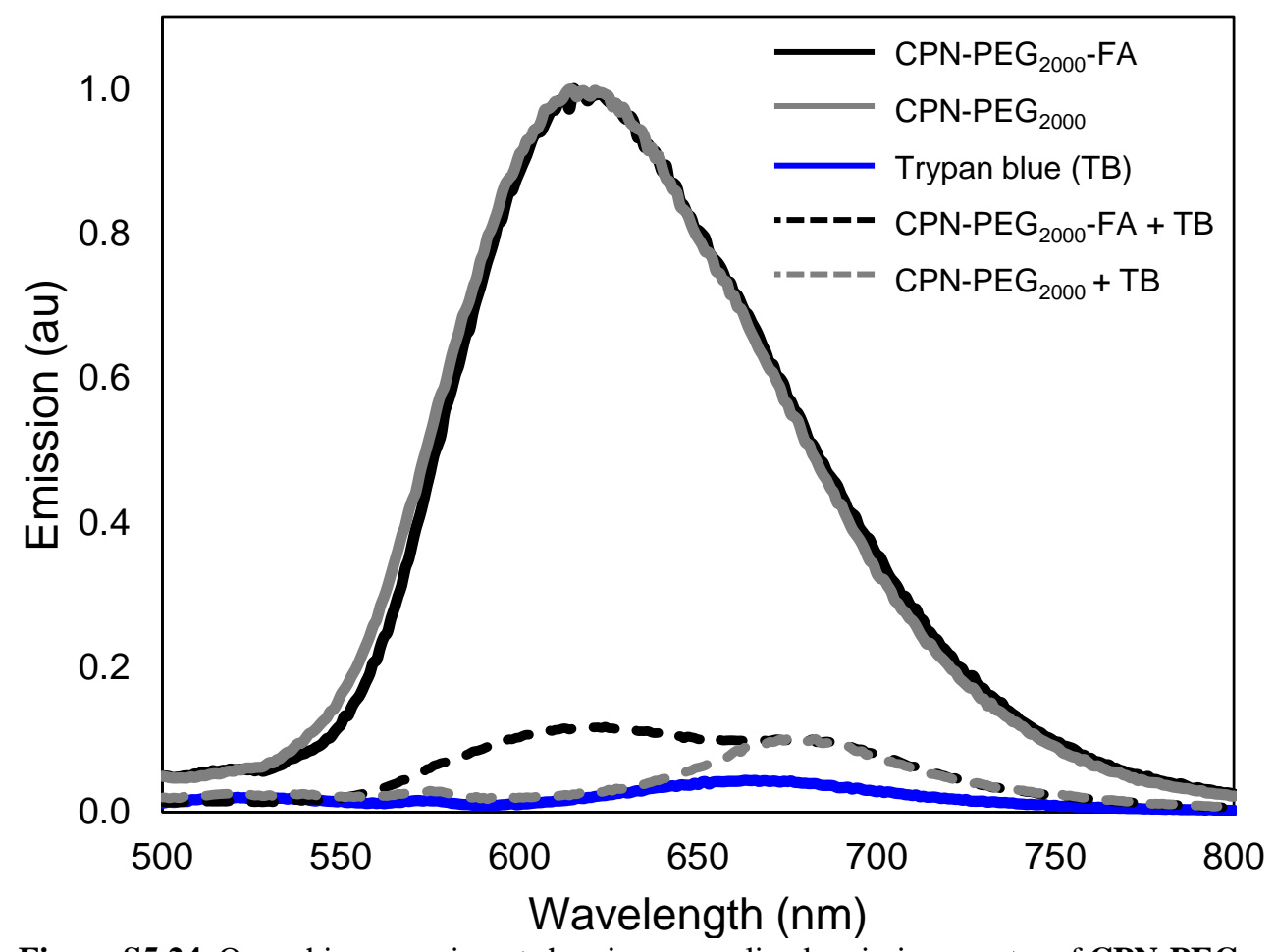

Figure S5.24. Quenching experiment showing normalized emission spectra of CPN-PEG 2000-FA and CPNPEG $_{2000}$ with (dashed lines) and without (solid lines) trypan blue (TB) treatment of $200 \mu \mathrm{M}$ in water for 10 minutes at $37^{\circ} \mathrm{C}$. 

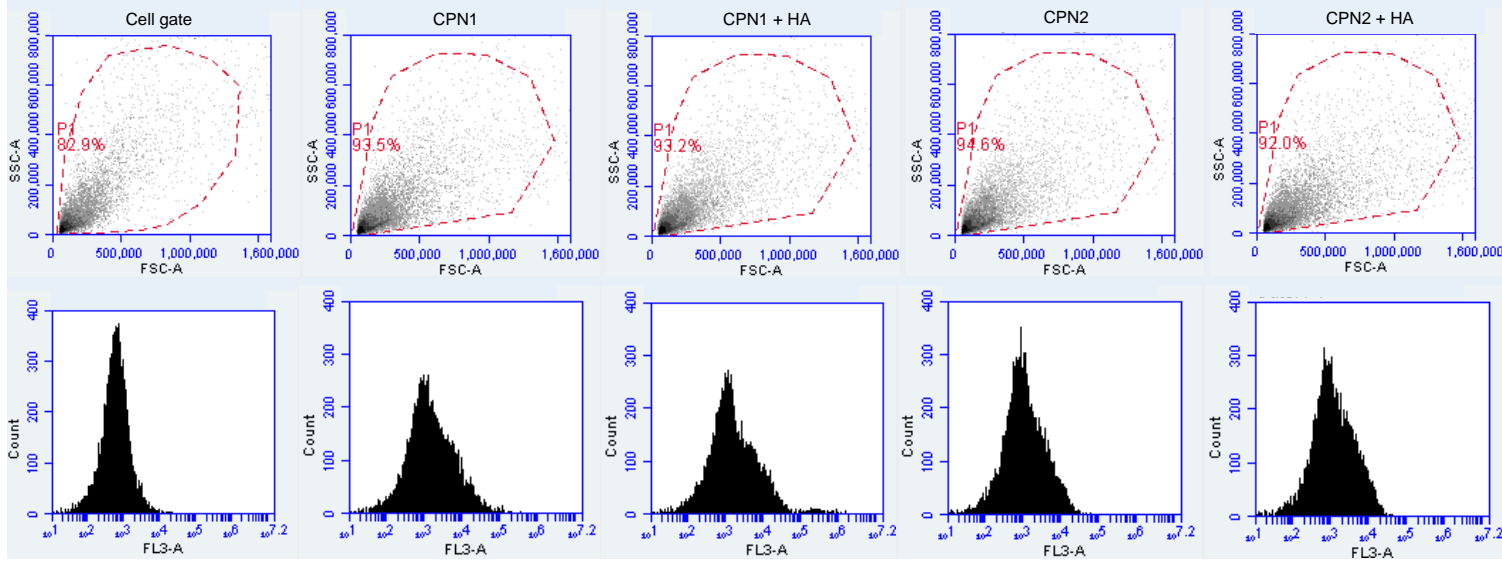

Figure S5.25. Representative scatterplots (top row) and intensity plots (bottom row) for optimized conditions for CPN-PEG $2000-\mathrm{FA} / \mathrm{HA}$ (8 h, 40K 1:1), and CPN-PEG $2000 / \mathrm{HA}$ (4 h, 40K 1:2). 10,000 events per measurement were recorded within the gate of control cells, which was selected based on forward and side scattering of control cells (top left) to eliminate data collection from dead cells and artifacts.

5.7 References

1. $\quad$ Zhu, C. L.; Liu, L. B.; Yang, Q.; Lv, F. T.; Wang, S. Chem. Rev. 2012, 112, 4687.

2. $\quad$ Pu, K. Y.; Li, K.; Liu, B. Adv. Funct. Mater. 2010, 20, 2770.

3. Traina, C. A.; Bakus, R. C.; Bazan, G. C. J. Am. Chem. Soc. 2011, 133, 12600.

4. $\quad$ Pu, K. Y.; Li, K.; Shi, J. B.; Liu, B. Chem. Mater. 2009, 21, 3816.

7. $\quad$ Disney, M. D.; Zheng, J.; Swager, T. M.; Seeberger, P. H. J. Am. Chem. Soc. 2004, 126, 13343.

5. $\quad$ Mao, S.; Shuai, X.; Unger, F.; Wittmar, M.; Xie, X.; Kissel, T.; Mao, S.

Biomaterials. 2005, 26, 6343-6356.

6. $\quad$ Kosaka, N.; Ogawa M.; Longmire, M. R.; Choyke, P. L.; Kobayashi, H. J. Biomed. Opt. 2009; 14, 14023-14035.

8. $\quad$ Kim, I.B.; Shin, H.; Garcia, A. J.; Bunz, U. H. F. Bioconjugate Chem. 2007, 18, 815.

9. $\quad$ Feng, X.; Lv, F.; Liu, L.; Tang, H.; Xing, C.; Yang, Q.; Wang, S. ACS Appl. Mater. Interfaces. 2010, 2, 2429-2435.

10. Z Zhu, C.; Liu, L.; Yang, Q.; Lv, F.; Wang, S. Chem. Rev. 2012, 112, 4687-4735.

11. Paulos, C. M.; Reddy, J. A.; Leamon, C. P.; Turk, M. J.; Low, P. S. Mol. Pharmacol. 2004, 66, 1406-1414.

12. Kim,; I. B. Shin, H.; Garcia, A. J.; Bunz, U. H. F. Bioconjugate Chem. 2007, 18, 815-820.

13. Ahmed, E.; Morton, S. W.; Hammond, P. T.; Swager, T. M. Adv. Mater. 2013, 25, 4504- 4510.

14. Almalik, A.; Karimil, S.; Ouasti, S.; Donno, R.; Wandrey, C.; Day, P. J.; Tirelli, N. Biomaterials. 2013, 34, 5369-5380. 
15. Liu, Y.; Sun, J.; Cao, W.; Yang, J.; Lian, H.; Li, X.; Sun, Y.; Wang, Y.; Wang, S.; He, Z. Int. J. Pharm. 2011, 421, 160- 169.

16. Zwicke, G. L.; Mansoori G. A., Jeffery, C. J. Nano Rev. 2012, 3, 18496-18507.

17. Choi, K. Y.; Min, K. H.; Yoon, H. Y.; Kim, K.; Park, J. H.; Kwon, I. C.; Choi, K.; Jeong. S. Y. Biomaterials. 2011, 32, 1880-1889.

18. Yue, T.; Zhang, X. ACS Nano. 2012, 6, 3196-3205.

19. Cerroni, B.; Chiessi, E.; Margheritelli, S.; Oddo, L.; and Paradossi, G. Biomacromolecules. 2011, 12, 593-601.

20. Sun, H.; Benjaminsen, R. V.; Almdal, K.; Andresen, T. L. Bioconjugate Chem. 2012, 23, 2247-2255.

21. Duncan, R.; Richardson, S. C. W. Mol. Pharmaceutics, 2012, 9, 2380-2402.

22. Van Dongen, M. A.; Dougherty, C. A.; Banaszak Holl, M. M. Biomacromolecules. 2014, 15, 3215-3234.

23. Sudimack, J.; Lee, R. L. Adv. Drug Deliv. Rev. 2000, 41, 147-162.

24. Lu, Y.; Low, P. S. Adv. Drug Deliv. Rev. 2012, 64, 342-352.

25. Mahou, P.; Zimmerley, M.; Loulier, K.; Matho, K. S.; Labroille, G.; Morin, X.; Supatto, W.; Livet, J.; Débarre, D.; Beaurepaire, E. Nature Methods. 2012, 9, 815- 818.

26. Pu, K.Y.; Li, K.; Liu, B. Chem. Mater. 2010, 22, 6736-6741.

27. Qhattal, H. S. S.; Liu, X. Mol Pharmacol. 2011, 8, 1233-1246.

28. Vokatá, T.; Moon, J. H. Macromolecules. 2013, 46, 1253-1259.

29. Twomey, M.; Na, Y.; Roche, Z.; Mendez, E.; Panday, N.; He, J.; Moon, J. H. Macromolecules. 2013, 46, 6374-6378.

30. Twomey, M.; Vokatá, T.; Kumar, M. R.; Moon, J. H. Chem. Comm. 2015, 51, 4065—4068.

31. Moon, J. H.;Mendez, E.; Kim, Y.;Kaura, A. Chem. Comm. 2011, 47, 8370-8372.

32. Trindade, A. F.; Frade, R. F. M.; Maçôas, E. M. S.; Graça, C.; Rodrigues, C. A. B.; Martinhoa, J. G.; Afonso, C. A. M. Org. Biomol. Chem. 2014, 12, 3181-3190.

33. Hed, J. FEMS Microbiol. Lett. 1977, 1, 357-361.

34. Van Strijp, J. A.; Van Kessel, K. P.; van der Tol, M. E.; Verhoef, J. J. Clin. Invest. 1989, 84, 107112.

35. Hed, J.; Hallden, G.; Johansson, S. G. O.; Larrson, P. J. Immunol. Methods. 1987, 101, 119-125.

36. Shi, F.; Sottile, J. J. Cell Sci. 2008, 121, 2360-2371. 


\section{CHAPTER VI}

General Conclusions

The development of CP-based biomaterials would provide a novel class of polymeric materials useful for a variety of applications. The use of CPNs has become of significant interest in biomedical applications as a consequence of their desirable properties including facile synthesis, high sensitivity, superior photostability, low cytotoxicity, and biocompatibility. The photophysical excellence and robustness of CP-based nanomaterials are particularly useful for labelling target cells. The synthetic versatility of CPs allows the easy modification of the conjugated backbone to tune emission and side chain structures to adjust biocompatibility through increased water solubility, controlled biodegradability, and incorporation of targeting. Structural modifications of CP side chains to incorporate ionic and hydrophilic moieties allow for tunable biophysical properties for improved biocompatibility and functionalized performance. Depending on the solubility of CP chains and the nature of interaction between CPs and analytes, structural changes can occur in individual CP chains or multiple chain aggregates, which correspond to changes in CP optical properties. These changes in optical properties present the opportunity of a CP-based sensor. Structural modifications result in changes in self-assembly and subsequent cellular interactions, which allows for a structure-function relationship to be established.

The second chapter presented a strategy for modulating particle shape using ionic complexation with HA in a backbone flexibility-dependent fashion. The incorporation of a semi-flexible, non-conjugated moiety into the backbone resulted in the formation of core-shell nanoparticles when a cationic CPN was treated with a polyanion compared to the control CPN with a rigid, fully conjugated backbone. The core-shell nanoparticles exhibited high specificity to cancer cells with low adsorption to normal cells, because of HA's affinity to overexpressed receptors on cancer cells. While in Chapter II, the semi-flexible CPN displayed enhanced targeting of cancer cells over normal cells, additional work showed there was no significant increase in internalization of the semi-flexible CPN, only changes to subcellular localization. Complexation of the semiflexible CPN with polyanions led to increased accumulation in different organelles compared to control polymers with rigid backbone. Differences in subcellular localization were expected based on the dramatic 
changes from spherical to core-sell particles. While ionic complexation led to increased labelling of cancer cells, a strategy to improve targeting and increase internalization of CPNs was explored in Chapter V using a dual ligand targeting approach.

The dramatic differences in ionic complexation determined by backbone flexibility led to the work in Chapter III. A systematic investigation on ionic complexation of CPNs that vary by side chain and backbone structure with biologically important anionic polysaccharides was conducted in the third chapter. A simple approach to differential sensing of biologically important molecules was presented. A systematic approach to understanding the effect of side chain and backbone structure was employed to establish a structure-function relationship. To improve differential sensing, an array of more structurally diverse CPNs could be utilized, which would lead to more dramatic differential interactions of CPNs and analytes. By developing structural diverse arrays, sensitivity can be greatly improved. Additional work using a controlled series of CPNs with PPE and PPB-type connectivity and incorporation of a flexible, non-conjugated linker displayed backbonedependent complexation with HA. The work in Chapters II and III presented how backbone flexibility affects ionic interactions, but subcellular localization studies would provide more information how exactly backbone connectivity affects cellular interactions, which was the explored in the fourth chapter

Chapter IV presented a strategy for mitochondrial targeting using the triphenylphosphonium (TPP) group that was dependent on the biodegradability and flexibility of the conjugated backbone. The subcellular localization and cellular toxicity were affected by backbone flexibility and molecular weight, in a timedependent fashion. The degradable, flexible linker allowed the CPN to be degraded to conjugated oligomers that could more easily diffuse after endocytosis and traffic to the mitochondria. The TPP-containing CPNs were treated with HA to improve solubility, and displayed modulated cellular interactions based on backbone structure. Additional work using a series of varying molecular weight nonflexible TPP-containing copolymers and flexible TPP-containing copolymers with varying flexible unit incorporation indicated that the presence of the flexible linker and at relatively higher percentage ( $>40 \%)$ was crucial for mitochondrial localization. This finding could be the result of the hydrophobicity of the polymer backbone, which causes aggregation in aqueous environment, and compromises the availability of the degradable linker to 
intracellular glutathione. A series of TPP-containing polymers that also incorporated the hydrogen peroxidesensitive moiety, aryl boronate, and degradable linker were synthesized. However, because of the hydrophobicity of this group and in combination with the bulky TPP group, dense aggregation was observed. To address these issues, additional more in-depth studies on the effect of the flexible linker for subcellular targeting are needed. In addition, improving the hydrophilicity of the TPP-containing CPNs would provide information as to how polymer solubility, CPN aggregation, and cellular uptake/trafficking can be modulated.

Chapter V detailed the synthesis of dual-targeting red emissive CPNs grafted with folic acid (FA) side chains and complexed with hyaluronic acid (HA). Modulation of uptake kinetics and amount of internalized CPNs was achieved by the dual ligand-targeting CPNs designed for folate and hyaluronan receptors. The grafting of $\mathrm{PEG}_{2000}$-FA side chain posed a synthetic challenge to control the amount of incorporation because of the poor solubility of FA. The synthetic approach would need to be modified to increase hydrophilicity to favor coupling of FA. Additional work is under way to use microwave-assisted polymerization, which would provide more reproducible results because of uniform heating and shorter reaction times. To assess accurately the dual ligand approach, a series of three CPNs are needed: CPN with PEG only side chain, CPN with PEGFA side chain, and CPN with PEG-FA complexed with HA. Since copolymers were used, a variation in copolymer blocks was obtained based on synthetic approach. A CPN array with controlled incorporation of FA to accurately assess cellular interactions of FA and HA would provide a clearer understanding of uptake pathways as function of the folate and hyaluronan receptors. Subcellular localization studies of targeting CPNs would provide information how modulating cell surface interactions affects subcellular trafficking and intracellular fate. 
VITA

\section{MEGAN TWOMEY}

Born Tampa, FL

$2006-2010$

B.S. in Chemistry with an emphasis in Forensic Science

Appalachian State University, Boone, NC, USA

Minor in Criminal Justice

$2011-2016$

$\mathrm{PhD}$ Candidate (expected graduation May 2016)

Florida International University, Miami, FL, USA

Dissertation title: Conjugated polymer-based biomaterials through controlled self-assembly

Advisor: Joong Ho Moon, Ph.D.

2012-2013

General Chemistry Outstanding Teaching Assistant Award

Florida International University, Miami, FL

2013-2014

General Chemistry Outstanding Teaching Assistant Award

Florida International University, Miami, FL

2015-2016

Dissertation Year Fellowship

Florida International University, Miami, FL

\section{PUBLICATIONS AND PRESENTATIONS}

LEE, J., Twomey, M., Machado, C., Gomez, G., Doshi, M., Gesquiere, A. J., Moon, J. H. "CaveolaeMediated Endocytosis of Conjugated Polymer Nanoparticles”, Macromolecular Bioscience (2013), 13: 913920.

TWOMEY, M., Moon, J. H., Poster Presentation, "Improved Small RNA Molecule Delivery Using Controlled Aggregation of Fluorescent Conjugated Polymers”, Materials Research Society Spring 2013 Meeting, Apr 1-5, 2013, San Francisco, CA.

TWOMEY, M., Na, Y., Roche, Z., Mendez, E.; Panday, N., He, J., Moon, J. H. "Fabrication of Core-Shell Nanoparticles via Controlled Aggregation of Semiflexible Conjugated Polymer and Hyaluronic Acid”, Macromolecules (2013), 46: 6374-6378.

TWOMEY, M., Vokata, T., Kumar, M. R., Moon, J. H., Poster Presentation, "Differential interactions of conjugated polymer nanoparticles with glycosaminoglycans in synthetic urine”, Materials Research Society Fall 2014 Meeting, Dec 1-5, 2014, Boston, MA. 
TWOMEY, M., Vokata, T., Kumar, M. R., Moon, J. H. "Differential interactions of conjugated polymer nanoparticles with glycosaminoglycans in synthetic urine”, Chemical Communications (2015), 51: 40654068.

VOKATA, T., Twomey, M., Mendez, E., Moon, J. H. Synthesis of Biodegradable Conjugated Polymers with Controlled Backbone Flexibility. Journal of Polymer Science A Polymer Chemistry (2015), 53: 1403-1412.

SHAN, Y., Panday, N., Myoung, Y., Twomey, M., Wang, Li, W., Celik, E., Moy, V., Wang, H., Moon, J. H., He, J. "Scanning ionic conductance microscopic study for cellular uptake of cationic conjugated polymer nanoparticles", Macromolecular Bioscience (2016), just accepted.

TWOMEY, M., Mendez, E., Manian, M. R., Moon, J. H. "Mitochondria-specific conjugated polymers nanoparticles”, Chemical Communications (2016), 52: 4910-4913.

TWOMEY, M.; Manandhar, P., Moon, J. H., Oral Presentation "Conjugated polymer nanoparticles for improved peptide-mediated drug delivery”, American Chemical Society Spring 2016 Meeting, Mar 13-17, 2016, San Diego, CA.

TWOMEY, M.; Manandhar, P., Moon, J. H. “Conjugated polymer nanoparticles for improved peptidemediated drug delivery", in preparation.

TWOMEY, M., Choudhury, R., Manandhar, P., Agoulnik, I. U., Moon, J. H. "Dual targeting conjugated polymer nanoparticles of folate and hyaluronan receptors for bioimaging”, in preparation. 